Joachim Nagel

\title{
Supply-Side Policy in den USA
}

Eine theoretische und empirische Analyse der angebotsorientierten Wirtschaftspolitik Reagans unter besonderer Berücksichtigung steuerlicher Aspekte 


\section{Joachim Nagel}

\section{Supply-Side Policy in den USA}

Ronald Reagan hat zu Beginn der 80er Jahre in den Vereinigten Staaten eine Wende zur angebotsorientierten Wirtschaftspolitik (Supply-Side Policy) eingeleitet. Im Rahmen dieser „neuen“ Wirtschaftspolitik wurden insbesondere die Spitzensteuersätze massiv gesenkt. Auf der konzeptionellen Grundlage der Laffer-Kurve sollten Anreizwirkungen für verstärkte Investitionen, höheres Sparen und ein größeres Arbeitsangebot ausgehen. Die Untersuchung zeigt, daß die Supply-Side Policy in den USA ihre makroökonomischen Ziele nicht erreicht hat. Die empirische Analyse der Grenzsteuersätze belegt, daß die untergeordneten Gebietskörperschaften in den USA auf die Steuersenkungen des Bundes mit Steuererhöhungen reagierten. Diese entgegengesetzte Steuerpolitik führte dazu, daß die Steuerbelastung insgesamt nicht gesenkt werden konnte. Ein weiteres Ergebnis ist, daß die Höhe der marginalen Steuersätze Zweifel aufkommen läßt, ob im Sinn der Laffer-Argumentation die Voraussetzungen für eine erfolgreiche Verwirklichung der Supply-Side Policy vorlagen.

Joachim Nagel wurde 1966 in Karlsruhe geboren. Er studierte von 1985 bis 1991 Volkswirtschaftslehre an der Universität Karlsruhe. Nach Abschluß seines Studiums arbeitete er dort als Wissenschaftlicher Angestellter am Institut für Wirtschaftspolitik und Wirtschaftsforschung (Sektion: Geld und Währung) und wurde 1997 promoviert. 
Supply-Side Policy in den USA

Joachim Nagel - 978-3-631-75238-8

Downloaded from PubFactory at 01/11/2019 06:47:33AM

via free access 


\section{FINANZWISSENSCHAFTLICHE SCHRIFTEN}

Herausgegeben von den Professoren

Albers, Krause-Junk, Littmann, Oberhauser, Pohmer, Schmidt

Band 81

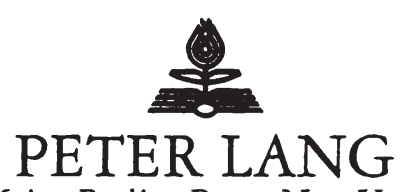

Frankfurt am Main - Berlin · Bern - New York - Paris - Wien 


\section{Joachim Nagel}

\section{Supply-Side Policy in den USA}

Eine theoretische und empirische Analyse der angebotsorientierten Wirtschaftspolitik Reagans unter besonderer Berücksichtigung steuerlicher Aspekte

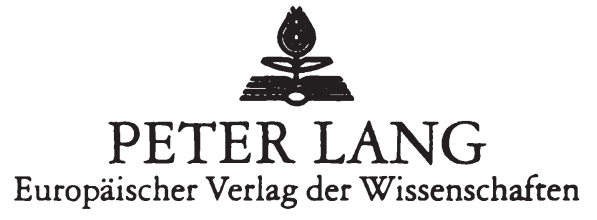


Die Deutsche Bibliothek - CIP-Einheitsaufnahme

Nagel, Joachim:

Supply-Side Policy in den USA : eine theoretische und empirische Analyse der angebotsorientierten Wirtschaftspolitik Reagans unter besonderer Berücksichtigung steuerlicher Aspekte / Joachim Nagel. - Frankfurt am Main ; Berlin ; Bern ; New York ; Paris ; Wien : Lang, 1997

(Finanzwissenschaftliche Schriften ; Bd. 81)

Zugl.: Karlsruhe, Univ., Diss., 1997

ISBN 3-631-31841-3

Open Access: The online version of this publication is published on www.peterlang.com and www.econstor.eu under the international Creative Commons License CC-BY 4.0. Learn more on how you can use and share this work: http://creativecommons. org/licenses/by/4.0.

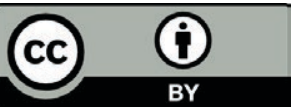

This book is available Open Access thanks to the kind support of ZBW - Leibniz-Informationszentrum Wirtschaft.

\author{
D 90 \\ ISSN 0170-8252 \\ ISBN 3-631-31841-3 \\ ISBN 978-3-631-75238-8 (eBook) \\ (c) Peter Lang $\mathrm{GmbH}$ \\ Europäischer Verlag der Wissenschaften \\ Frankfurt am Main 1997 \\ Alle Rechte vorbehalten.
}

Das Werk einschließlich aller seiner Teile ist urheberrechtlich geschützt. Jede Verwertung außerhalb der engen Grenzen des

Urheberrechtsgesetzes ist ohne Zustimmung des Verlages unzulässig und strafbar. Das gilt insbesondere für Vervielfältigungen, Übersetzungen, Mikroverfilmungen und die Einspeicherung und Verarbeitung in elektronischen Systemen.

Printed in Germany 123457 


\section{Inhaltsverzeichnis}

Abbildungsverzeichnis ...................................................................................

Graphikverzeichnis ......................................................................................... 9

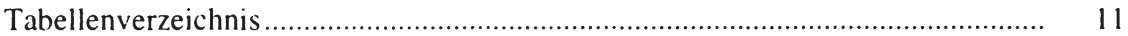

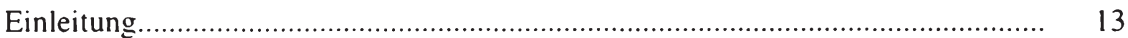

Anmerkungen zum Aufbau der Arbeit ......................................................... 15

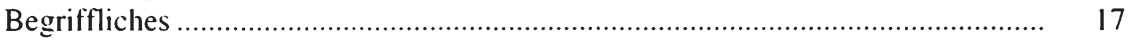

1 Die Ausgangssituation in den Vereinigten Staaten - Voraussetzung für die Verwirklichung der Supply-Side Policy ......................................................... 19

1.1 Die Nachkriegsjahre ............................................................................. 19

1.2 Die wirtschaftliche Entwicklung in den 70er Jahren.................................... 21

1.2.1 Wirtschaftliche Auswirkungen des Vietnam-Krieges......................... 23

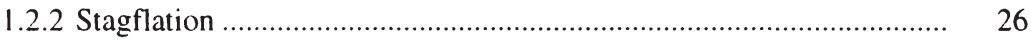

1.2 .3 Ölpreiskrisen ............................................................................ 28

1.2.4 Technologische und soziologische Veränderungen - Gründe für die Wirtschaftskrise

$\mathrm{Zu}$ den technologischen Gründen: .................................................... 31

$\mathrm{Zu}$ den soziologischen Gründen:.................................................... 31

1.3 Die Krise der Nachfragetheorie ............................................................... 34

1.4 Wirtschaftspolitische Veränderungen........................................................... 35

1.5 Resümee ....................................................................................... 39

2 Die Theorie der Supply-Side Policy ................................................................. 41

2.1 Die Supply-Sider - Akteure des wirtschaftspolitischen Wandels.................. 41

2.2 Die theoretischen Grundlagen der Supply-Side Policy ................................ 44

2.2.1 Ursprünge der Supply-Side Policy: Der klassische Liberalismus........ 47

2.2.2 Ursprünge der Supply-Side Policy: Der klassische Preismechanis-

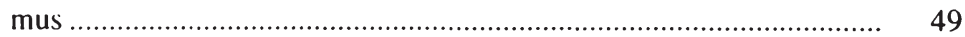

2.2.3 Ursprünge der Supply-Side Policy: Das Saysche Theorem ................. 51

2.2.4 Annahmen der klassischen Theorie - Fundament der Supply-Side Policy.

2.2.5 Die Laffer-Kurve............................................................................. 54

2.2.5.1 Anmerkungen zur Laffer-Kurve ........................................ 54

2.2.5.2 Verlauf und Beschreibung der Laffer-Kurve....................... 55

2.2.5.3 Tobin-Herleitung der Laffer-Kurve ..................................... 57

2.2.6 Das Wedge-Modell ........................................................................ 62 
2.2.7 Das Iso-Produktions-/Einnahmen-Modell.............................................. 63

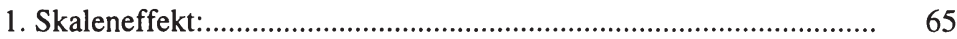

2. Substitutionseffekt:................................................................... 65

2.2.8 Auswirkungen der angebotsorientierten Steuerpolitik auf die Ange-

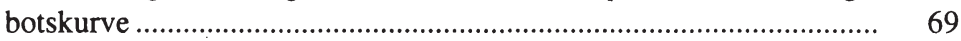

2.3 Kritik an der Theorie der Supply-Side Policy ............................................ 70

2.3.1 Steuerwirkungen auf dem Arbeitsmarkt ............................................ $\quad 72$

2.3.2 Steuersenkung und Kapitalangebot................................................. $\quad 74$

2.3.3 Einflußfaktoren auf das Investitionsverhalten ...................................... $\quad 75$

2.3.4 Kritische Bemerkungen zur Laffer-Kurve ......................................... $\quad 76$

2.3.5 Die Trägheit angebotspolitischer Erfolge ......................................... 78

2.3.6 Nachfrageeffekte einer angebotsorientierten Steuerpolitik ................. $\quad 78$

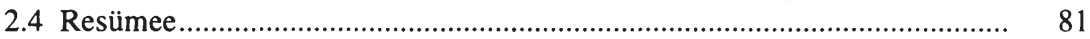

3 Reaganomics....................................................................................... 83

3.1 Das Reformprogramm …………………………................................... 83

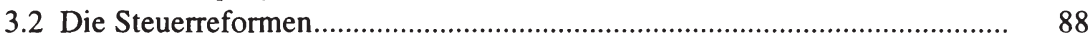

3.2.1 Die Steuerreform von 1981 (ERTA) …………............................... 89

3.2.2 Die Steuerreformen: TEFRA, SSA und DEFRA ................................ $\quad 90$

3.2.3 Die Steuerreform von 1986 (TRA) ................................................ 93

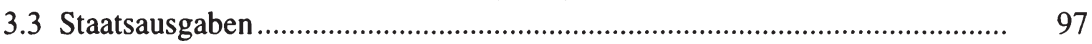

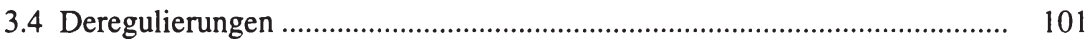

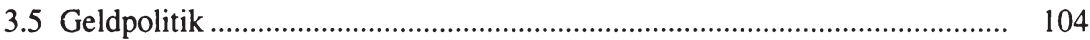

3.6 Die Entwicklung des Dollars und das Zwillingsdefizit .............................. 108

3.7 Die Reagan-Projektion und was daraus wurde............................................. 114

3.8 Die langfristigen ökonomischen Auswirkungen der Reaganomics............... 116

3.8.1 Wirtschaftswachstum .................................................................. 116

3.8.2 Investitionen und Sparen ............................................................. 120

3.8.3 Arbeitsangebot, Arbeitsmarkt und Einkommensentwicklung ............ 123

3.8.4 Haushaltsentwicklung .......................................................................... 125

3.8.5 Wirtschaftliche Rahmenbedingungen .......................................... 128

3.9 Resümee

4 Schätzung des marginalen Steuersatzes für die U.S.A....................................... 133

4.1 Ansatz für die Schätzung ..................................................................... 135

4.2 Vorgehen bei der Schätzung .................................................................. 135

4.2.1 Die Regressionsgleichung ................................................................ 135

4.3 Ergebnisse der Schätzung des marginalen Steuersatzes................................ 136

4.4 Interpretation der Ergebnisse ................................................................... 139

5 Ergebniszusammenfassung und Schlußbemerkung........................................... 143

Literaturverzeichnis ......................................................................................... 149 


\section{Abbildungsverzeichnis}

Abbildung 1: Entwicklung. Veränderung und Einflußfaktoren der wirtschaftlichen Entwicklung in den Vereinigten Staaten in den 70er Jah-

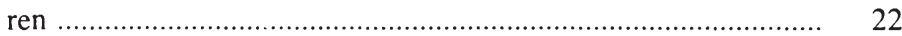

Abbildung 2: $\quad$ Bedeutende Supply-Sider............................................................ 45

Abbildung 3: Die Laffer-Kurve.................................................................. 56

Abbildung 4: $\quad$ Das Wedge-Modell ……………………………….................. 62

Abbildung 5: Iso-Produktionskurven ............................................................. 64

Abbildung 6: Iso-Einnahmen Kurve ……………………………………..... 66

Abbildung 7: Der optimale Steuer-Mix........................................................... 68

Abbildung 8: $\quad$ Supply-Side Policy, Steuersenkung und gesamtwirtschaftliches

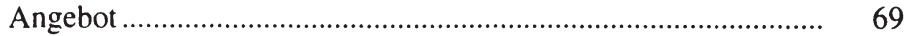

Abbildung 9: Supply-Side Policy, Steuersenkung, gesamtwirtschaftliches Angebot und Nachfrage, Szenario 1 ............................................. $\quad 79$

Abbildung 10: Supply-Side Policy. Steuersenkung, gesamtwirtschaftliches Angebot und Nachfrage. Szenario 2 
Joachim Nagel - 978-3-631-75238-8

Downloaded from PubFactory at 01/11/2019 06:47:33AM

via free access 
Graphikverzeichnis

Graphik 1: Reales Einkommen, Arbeitsproduktivität und Reallohnentwicklung 20

Graphik 2: Arbeitslosigkeit und Inflation in den 60er und 70er Jahren ............. Graphik 3: Durchschnittliches jährliches Wachstum des Bruttosozialprodukts pro Beschäftigtem in den großen Industrienationen, 1964-1978, (in \%)

Graphik 4: Antizyklische Wirtschaftspolitik in den Vereinigten Staaten, 1960 bis 1979

Graphik 5: Budgetdefizit der Vereinigten Staaten in den Jahren 1981 bis 1986 Verteidigungsausgaben der Vereinigten Staaten in den Jahren 1981 bis 1986

Graphik 7: Struktur des Bundeshaushaltes der Vereinigten Staaten in den Jahren 1981 und 1986

Graphik 8: DM/US-Dollar - Wechselkurs in den Jahren 1970 bis 1992

Graphik 9: Zins- und Wechselkursentwicklung in den Vereinigten Staaten in den Jahren 1970 bis 1992

Graphik 10: Handelsbilanzdefizit der Vereinigten Staaten in den Jahren 1980 bis 1992

Graphik 11: Zwillingsdefizit der Vereinigten Staaten - Entwicklung in den Jahren 1980 bis 1992

Graphik 12: Wirtschaftliche Entwicklung in den Vereinigten Staaten von 1960 bis 1992

Graphik 13: Entwicklung der Produktivität in den Vereinigten Staaten in den Jahren 1960 bis 1992

Graphik 14: Investitions- und Sparentwicklung in den Vereinigten Staaten in den Jahren 1960 bis 1992.

Graphik 15: Arbeitslosenquote in den Vereinigten Staaten in den Jahren 1960 bis 1992. in \% 
Joachim Nagel - 978-3-631-75238-8

Downloaded from PubFactory at 01/11/2019 06:47:33AM

via free access 


\section{Tabellenverzeichnis}

Tabelle 1: Verteidigungsausgaben in Anteilen am Bruttoinlandsprodukt in den Vereinigten Staaten in den 60er Jahren (in \%)

Tabelle 2: Geldmengenwachstum M1 in den Vereinigten Staaten, 1960 - 1979. ausgewählte Zeiträume (in \%)

Tabelle 3: Vergleich der wirtschaftlichen Entwicklung der 60er und 70er Jahre in den Vereinigten Staaten

Tabelle 4: Durchschnittliches jährliches Wachstum des Bruttosozialprodukts pro Beschäftigtem in den großen Industrienationen, 1964 - 1978, (in \%)

Tabelle 5: Durchschnittliche jährliche Zunahme des zivilen Arbeitskräfteangebots in den Vereinigten Staaten, 1960 - 1979, ausgewählte Zeiträume, (in \%)

Tabelle 6: Grenzsteuersätze der Einkommenssteuer in Abhängigkeit der Einkommenshöhe für einen Vier-Personen Haushalt in den Vereinigten Staaten, ausgewählte Zeiträume, (in \%).

Tabelle 7: Steuer-. Staats-, Konsum- und Investitionsquote in Anteilen am Bruttoinlandsprodukt in den Vereinigten Staaten, 1960 - 1979, ausgewählte Zeiträume, (in \%).

Tabelle 8: Referenzgrößen der wirtschaftlichen Entwicklung, ausgewählte Zeiträume, (in \%).

Tabelle 9: Die ökonomische Ausgangssituation für Ronald Reagan im Jahr 1980 im Vergleich mit den 70er Jahren, (in \%).

Tabelle 10: Die Steuerreformen während der Präsidentschaft Ronald Reagans ...

Tabelle 11: Die maximalen Grenzsteuersätze der Einkommensteuer des Bundes in den Vereinigten Staaten, 1946 - 1992, (in \%).

Tabelle 12: Erwartete Staatseinnahmen auf der Basis der Steuerreform, in Anteilen des nominalen Bruttosozialprodukts (GNP), 1981 - 1986, (in \%).

Tabelle 13: Geplante Staatsausgaben und projizierter Budgetsaldo auf der Basis der Steuerreform. 1981 bis 1986, in Mrd. Dollar.

Tabelle 14: Erwartete Staatsausgaben auf der Basis der Steuerreform, in Anteilen des nominalen Bruttosozialprodukts (GNP), 1981 - 1986. (in \%).

Tabelle 15: Erwartete Staatseinnahmen (incl. Beiträge zu den Sozialversicherungen) und erwartete Entwicklung des nominalen Bruttosozialprodukts (GNP) auf der Basis der Steuerreform, 1981 bis 1986, in Mrd. Dollar.....

Tabelle 16: Die wirtschaftliche Situation der Vereinigten Staaten in den Jahren 1981 und 1982. 
Tabelle 17: Die Entwicklung des Budgetdefizites der Vereinigten Staaten in Relation zum Bruttoinlandsprodukt in den Jahren 1981 bis 1984.

Tabelle 18: Die Geldpolitik der amerikanischen Zentralbank in den Jahren 1978 bis 1983

Tabelle 19: Wirtschaftswachstum, Zinsen, Budgetentwicklung und Preisniveau in den Vereinigten Staaten im Vergleich der Jahre 1975 bis 1980 und 1981 bis 1986, in \%

Tabelle 20: Die tatsächliche wirtschaftliche Entwicklung in den Vereinigten Staaten im Vergleich mit der Reagan - Projektion, in Mrd. US-Dollar und in \%

Tabelle 21: Das durchschnittliche reale Wirtschaftswachstum in den Vereinigten Staaten, 1960 bis 1969, 1970 - 1981, 1960 - 1992 und in der Zeit der Reaganomics 1982 bis 1992, in \%

Tabelle 22: Produktivitätsentwicklung in den Vereinigten Staaten, 1960 bis 1969, 1970 - 1981, 1960 - 1992 und in der Zeit der Reaganomics 1982 bis 1992 , in \%

Tabelle 23: Investitionsentwicklung und Sparquote in den Vereinigten Staaten, 1960 bis 1969, 1970 - 1981, 1960 - 1992 und in der Zeit der Reaganomics 1982 bis 1992, in \%

Tabelle 24: Entwicklung des Konsums in den Vereinigten Staaten, 1960 bis 1969, 1970 - 1981, 1960 - 1992 und in der Zeit der Reaganomics 1982 bis 1992, in \%

Tabelle 25: Entwicklung des Arbeitsmarktes in den Vereinigten Staaten 1960 bis 1969, 1970 - 1981, 1960 - 1992 und in der Zeit der Reaganomics 1982 bis 1992, in \%

Tabelle 26: Entwicklung des Bundeshaushaltes in den Vereinigten Staaten, 1960 bis 1969, 1970 - 1981, 1960 - 1992 und in der Zeit der Reaganomics 1982 bis 1992, in \%

Tabelle 27: Entwicklung der Steuereinnahmen des Bundes und der Bundesstaaten und der Gemeinden in den Vereinigten Staaten, 1960 bis 1969 , 1970 - 1981, 1960 - 1992 und in der Zeit der Reaganomics 1982 bis 1992 , in \%

Tabelle 28: Zinsentwicklung und Inflation in den Vereinigten Staaten, 1960 bis 1969, 1970 - 1981, 1960 - 1992 und in der Zeit der Reaganomics 1982 bis 1992, in \%

Tabelle 29: Entwicklung des Außenhandels und des Dollarkurses in den Vereinigten Staaten, 1972 bis 1981 und in der Zeit der Reaganomics 1982 bis 1992 , in \% und DM

Tabelle 30: Schätzergebnisse für die marginale Steuerquote in den Vereinigten Staaten

Tabelle 31: Die durchschnittliche Steuerquote in den Vereinigten Staaten 


\title{
Einleitung
}

\begin{abstract}
"Sie erläuterten ihm die Laffer-Kurve. Er nahm es auf wie eine Symphonie. Er wußte sofort, daß diese Leute recht hatten, und hat auch später nie einen Augenblick daran gezweifelt. Schließlich hatte er sich selbst einmal auf der Laffer-Kurve befunden.

Im Zweiten Weltkrieg habe ich im Film das große Geld gemacht. erzählte er oft. Damals betrug der kriegsbedingte Zuschlag zur Einkommensteuer bis zu neunzig Prozent. Man konnte nur vier Filme machen, dann war man schon in der höchsten Steuerklasse, erzählte er weiter. Deshalb hörten wir alle nach vier Filmen auf zu arbeiten und fuhren aufs Land." 1
\end{abstract}

Zur Natur des Verhaltens der Menschen gehört seit jeher die Abkehr von Strategien, die über einen längeren Zeitraum zwar gut funktionierten, aber für neu auftauchende Probleme keinen unmittelbaren Lösungsweg offenbarten. Mitte der 70er Jahre hatte die Weltwirtschaft eine solche Phase des Umbruchs von einer Strategie zu einer anderen erfahren. Die bis dahin von den meisten Industriestaaten praktizierte volkswirtschaftliche Problemlösungsstrategie einer nachfrageorientierten Wirtschaftspolitik, der sogenannte Keynesianismus, hatte ausgedient. Den Problemen hohe Arbeitslosigkeit, Inflation und geringes Wirtschaftswachstum, die zu diesem Zeitpunkt die Volkswirtschaften gleichzeitig belasteten, stand die nachfrageorientierte Wirtschaftspolitik mehr oder weniger ratlos gegenüber.

Den „Rettungsanker“ für die kränkelnden Volkswirtschaften sahen einige Politiker, insbesondere in den Vereinigten Staaten und Großbritannien, in einer angebotsorientierten Wirtschaftspolitik, der sogenannten Supply-Side Policy. Die Supply-Side Policy schien in dieser schwierigen ökonomischen Situation die Lösung parat zu haben. Die angebotsorientierte Wirtschaftspolitik ist keine substantiell neue Politik, sondern basiert auf Elementen der klassischen Wirtschaftstheorie.

Das Kernstück der Supply-Side Theorie ist die Annahme, daß über zusätzliche ökonomische Leistungsanreize die Angebotsbedingungen verbessert werden und dadurch die individuelle Wirtschaftsaktivität ausgeweitet wird. Das wirtschaftliche Aktivitätsniveau des Individuums wird zur zentralen Referenzgröße für die wirtschaftliche Entwicklung einer Volkswirtschaft. Liegt eine Wachstumsschwäche der Ökonomie vor, dann sei dies ein Hinweis dafür, daß nicht genügend Anreize bestehen, um Leistung zu entfalten, zu produzieren und Einkommen zu schaffen.

Die Ursache für makroökonomische Probleme sind in der Sicht der Supply-Side Policy unzureichende mikroökonomische Anreize. ${ }^{2}$

1 Vgl. Stockman. D.. Der Triumph der Politik, Die Krise der Reagan-Regierung und ihre Auswirkung auf die Weltwirtschaft. München 1986, S. 17.

2 Vgl. Filc, W.. Monetäre Fundierung einer angebotsorientierten Stabilitätspolitik. in: "Wirtschaftspolitische Diskurse" Reihe 37 des Forschungsinstituts der Friedrich-Ebert-Stiftung. 25 Jahre 
Der Versuch einer konsequenten Umsetzung der Supply-Side Theorie in die ökonomische Praxis einer Volkswirtschaft wurde mit dem Amtsantritt von Ronald Reagan zum Präsidenten der Vereinigten Staaten im Jahr 1981 eingeleitet. Diese Grundausrichtung der amerikanischen Wirtschaftspolitik wurde - grosso modo - unter der Präsidentschaft Bush's bis zum Jahr 1992 beibehalten.

Das ökonomische Programm der Reagan-Administration, das der neu gewählte Präsident einen Monat nach seinem Amtsantritt dem amerikanischen Kongreß vorlegte. war von Beginn an auf eine Stärkung der ökonomischen Leistungsanreize ausgerichtet.

Im Mittelpunkt der Strategie dieser Wirtschaftspolitik steht die Leistungsfähigkeit des Menschen. Diese Leistungsfähigkeit gilt es, im Rahmen der Ausgestaltung der Supply-Side Policy zu stärken. Derjenige, der mehr leisten möchte, soll dafür nicht vom Staat über eine höhere Steuerbelastung bestraft werden. Dies machten die Verfechter einer Supply-Side Policy der Wirtschaftspolitik, vornehmlich in den U.S.A., in den 70er Jahren zum Vorwurf. Das Konzept der angebotsorientierten Wirtschaftspolitik erschien erfolgversprechend. Die individuelle Grenzsteuerbelastung senken, die stärkste Forderung der Angebotstheoretiker, damit der Anreiz zur zusätzlichen Leistung gegeben ist, wurde zum politischen „Knüller“. Steuersenkungen lassen sich im Wahlkampf besonders gut verkaufen. Das erkannte Ronald Reagan, der als republikanischer Präsidentschaftsbwerber im Wahlkampf 1980 gegen den demokratischen Präsidenten Carter antrat. Die Forderungen der Supply-Side Policy wurden zum Markenzeichen der Wirtschaftspolitik Ronald Reagans. Nach seinem Wahlsieg hielt er an dem Wirtschaftskonzept fest und setzte massive Steuersenkungen durch. Die Angebotspolitik fand in der Reaganschen Ausprägung eine zusätzliche Begriffsfindung, die sogenannte Reaganomics.

Stabilitätsgesetz: Überlegungen zu einer zeitgerechten Ausgestaltung der Stabilitäts- und Wachstumspolitik, S. 25-49. 
Der Ansporn zu dieser Arbeit resultierte aus der Idee, die angebotsorientierte Wirtschaftspolitik am Beispiel der Reaganomics zu analysieren.

Für diese Untersuchung erschien es zunächst sinnvoll, die Voraussetzungen zu beschreiben, die dazu führten, daß die Supply-Side Policy zum Leitbild der amerikanischen Wirtschaftspolitik der 80er Jahre wurde. Im 1. Kapitel wird die wirtschaftliche und politische Ausgangssituation der Vereinigten Staaten beschrieben, die die Voraussetzung für die Verwirklichung der Supply-Side Policy bildete. Die Ausarbeitung gibt dabei einen Überblick über Gründe, die als Erklärung für das Aufkommen der angebotsorientierten Wirtschaftspolitik herangezogen werden können.

Im 2. Kapitel steht die Theorie der Supply-Side Policy im Mittelpunkt der Ausarbeitung. Von besonderem Interesse ist hierbei die Frage, wie die Supply-Side Theorie im Rahmen der Wirtschaftstheorien einzuordnen ist. Das 2. Kapitel macht die enge Anlehnung der Supply-Side Policy an die klassische Wirtschaftstheorie deutlich.

Ein weiterer Schwerpunkt der theoretischen Analyse ist die Laffer-Kurve. Sie stellt das Fundament der Supply-Side Policy dar. Besonderen Stellenwert erhält bei der Beschreibung der Laffer-Kurve die Tobin-Herleitung des Kurvenverlaufs. Diese Herleitung ist im Hinblick auf die Einschätzung, ab wann ein Steuersatz im Sinn der LafferArgumentation gesenkt werden muß, relevant.

Das 2. Kapitel arbeitet mit einigen wörtlichen Zitaten zur Supply-Side Policy. Die Literaturquellen sollen verdeutlichen, inwieweit die Angebotstheorie den Ausgangspunkt für die Reaganomics bildete und wo der Ursprung und Ausgangspunkt der Theorie lag.

Am Ende des 2. Kapitels wird die Supply-Side Policy einer kritischen Analyse unterzogen.

Die Reaganomics wird im Rahmen des 3. Kapitels beschrieben. Die Ausführungen zur Reaganomics sollen belegen, daß diese Wirtschaftspolitik sich die Annahmen der Supply-Side Policy zu eigen machte. Die fundamentalen Reformschritte in der Wirtschaftspolitik, die hierzu während der 80er Jahre in den U.S.A. unternommen wurden, werden analysiert. Diese Darstellung geht im 3. Kapitel über in die Untersuchung der wirtschaftlichen Lage der Vereinigten Staaten in der Zeit der Reaganomics. Die wirtschaftliche Entwicklung wird dabei auf dem Hintergrund des sich ergebenden PolicyMix aus Geld- und Fiskalpolitik diskutiert.

Zum Abschluß des 3. Kapitels steht die quantitative Analyse der langfristigen wirtschaftlichen Ergebnisse der Reaganomics im Mittelpunkt der Arbeit. Der Zeitraum von 1982 bis 1992 wird in Bezug zu wichtigen makroökonomischen Referenzgrößen mit den wirtschaftlichen Resultaten anderer Perioden verglichen. Für diese Untersuchung 
lag eine konsistente Datenbasis - über einen längeren Zeitraum vergleichbare volkswirtschaftliche Kenngrößen - für den Zeitraum von 1960 bis 1992 vom „U.S. Department of Commerce" und vom „Economic Report of the President" vor.

Im 4. Kapitel werden auf der Basis der vorliegenden Quartalswerte für die Steuereinnahmen und das nominale Bruttoinlandsprodukt des „U.S. Department of Commerce" über eine Regression die marginalen Steuerquoten der Vereinigten Staaten für den Zeitraum von 1960 bis 1992, differenziert nach Gebietskörperschaften, geschätzt. Diese Schätzung greift das zentrale Element der Supply-Side Policy, die marginale Steuerquote, auf und zeigt an den Ergebnissen des empirischen Ansatzes, warum die angebotsorientierte Wirtschaftspolitik nicht gegriffen hat.

Das 5. Kapitel faßt die wichtigsten Ergebnisse der Ausarbeitung zusammen. Die Schätzung der marginalen Steuerquoten wird hierbei als Argumentationshilfe für ein Plädoyer für die Koordination von Geld- und Fiskalpolitik und der Verknüpfung von angebots- und nachfrageorientierten Elementen innerhalb der Wirtschaftspolitik verwendet. 


\section{Begriffliches}

Wie schon in der Einleitung und in den Anmerkungen zum Aufbau der Arbeit geschehen, werden die Begriffe Supply-Side Policy und angebotsorientierte Wirtschaftspolitik im Rahmen der Arbeit synonym verwendet. Der Oberbegriff für die angebotsorientierte Wirtschaftspolitik am Beispiel der Vereinigten Staaten ist die Bezeichnung Reaganomics. 
Joachim Nagel - 978-3-631-75238-8

Downloaded from PubFactory at 01/11/2019 06:47:33AM

via free access 
1 Die Ausgangssituation in den Vereinigten Staaten - Voraussetzung für die Verwirklichung der Supply-Side Policy

In diesem Kapitel wird die Ausgangssituation in den Vereinigten Staaten Ende der 70er Jahre beschrieben, die die Voraussetzung für die Verwirklichung der Supply-Side Policy in den 80er Jahren bildete. Es wird auf die Nachkriegsjahre eingegangen, die allgemein nur von geringen Schwankungen der Konjunktur gekennzeichnet waren. In den 70er Jahren ließ sich die Einschätzung eines stetigen und störungsfreien Wachstums nicht mehr aufrecht erhalten. Diese Entwicklung traf die U.S.A. besonders hart. Die bis dahin in der Wirtschaftspolitik bevorzugte Theorie des Keynesianismus schien auf die neue wirtschaftliche Entwicklung keine geeigneten Antworten zu haben. Der Weg für eine "neue" Theorie, die Einzug in die Wirtschaftspolitik halten konnte, war frei.

\subsection{Die Nachkriegsjahre}

Nach dem 2. Weltkrieg setzte in den U.S.A. eine wirtschaftliche Boomphase ein. Bis zum Jahr 1973 hielt das überdurchschnittlich starke Produktivitätswachstum an. In der Periode von 1947 bis zum Jahr 1973 betrug die durchschnittliche jährliche Wachstumsrate der Arbeitsproduktivität in den Vereinigten Staaten 3,1 Prozent. ' Für den Zeitraum von 1900 bis 1973 lag der Zuwachs im Durchschnitt bei 2 Prozent. Eine zweiprozentige Wachstumsrate verdoppelte die Produktivität innerhalb von 35 Jahren. Nach dem zweiten Weltkrieg benötigte die Volkswirtschaft der Vereinigten Staaten hierzu weniger als 25 Jahre.

Ein starker Anstieg der Arbeitsproduktivität bleibt über einen längeren Zeitraum betrachtet nicht ohne Auswirkungen auf die Entwicklung des Lebensstandards. Ein stetiger Anstieg der Produktivität versetzt die Wirtschaft in die Lage, bei gleichzeitig steigenden Reallöhnen, zusätzliche Arbeitsplätze zu schaffen.

Vergleichbar der Arbeitsproduktivitätszunahme in den Vereinigten Staaten, entwikkelte sich auf lange Sicht auch der Lebensstandard der amerikanischen Bevölkerung in diesem Zeitraum überaus positiv.

Im Jahreswirtschaftsbericht 1994 des amerikanischen Präsidenten (Economic Report of the President) wurde als Referenzgröße für die Entwicklung des Lebensstandards in den U.S.A. im Zeitraum von 1947 bis 1992 das reale mittlere Familieneinkommen (Real Median Family Income) verwendet. Für die Produktivitätsentwicklung wurde die Produktion pro Arbeitsstunde (Output per Hour) zugrundegelegt; die Lohnentwicklung (Real Compensation per Hour) wurde an der durchschnittlichen realen Entlohnung für

1 Vgl. Economic Report of the President, Februar 1994, S. 24. 
eine Arbeitsstunde gemessen. Das Schaubild (Graphik 1) für die Referenzgrößen Produktivität, reales Familieneinkommen und Reallohnentwicklung für die Periode 1947 bis 1992 zeigt, daß der Verlauf der Entwicklung des realen Familieneinkommens und des Reallohns in den Vereinigten Staaten annähernd gleichgerichtet war. Dieses Ergebnis ist einsichtig, denn mittelfristig kann nur etwas zusätzlich verteilt werden, was auch tatsächlich zusätzlich erarbeitet wurde.

"It is only steady productivity gains that enable the economy to generate more jobs and rising real wages at the same time." 2

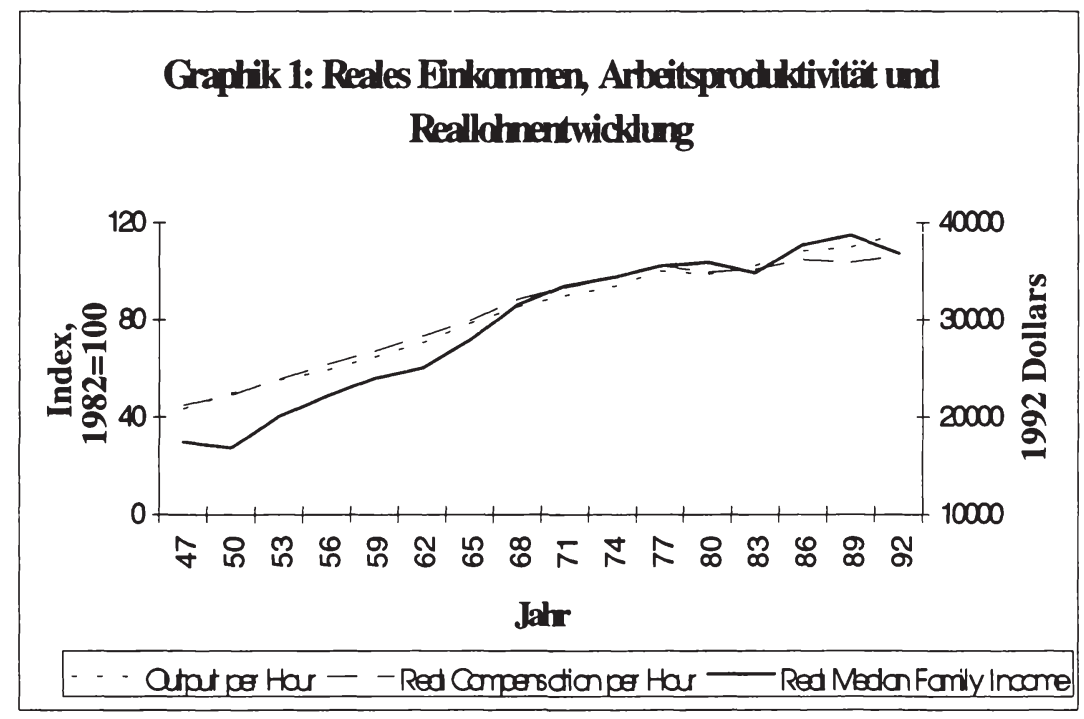

Quelle: Economic Report of the President, 1994, S.304, 322, eigene Darstellung.

Der amerikanische Wirtschaftswissenschaftler Paul Krugman beschreibt die wirtschaftliche Entwicklung in den Vereinigten Staaten in den ersten beiden Jahrzehnten nach dem 2. Weltkrieg folgendermaßen:

"We had become a middle-class nation, in which 63 percent of families owned their own homes, in which there was as many private cars as families, in which only 10 percent of families were still in poverty." 3

2 Ebenda, S. 24.

3 Vgl. Krugman, P., Peddling Prosperity: eonomic sense and nonsense in the age of diminished expectations, New York-London 1994, S. 57. 
Ende der 60er Jahre wurde in den U.S.A. allgemein davon ausgegangen, da $\beta$ die positive wirtschaftliche Entwicklung mit einem starken Produktivitätswachstum anhalten werde. Für die meisten Ökonomen war Ende 1969 keine Rezession in Sicht. Sie waren vielmehr der Auffassung, da $B$ die Phase des störungsfreien Wirtschaftswachstums noch nicht zu Ende sei. ${ }^{4}$ Diese Einschätzung sollte sich als falsch erweisen.

\subsection{Die wirtschaftliche Entwicklung in den 70er Jahren}

Zu Beginn der 70er Jahre war, trotz einiger kleiner Rückschläge, wirtschaftliche Stabilität als Ausgangspunkt für einen weiteren, ungebremsten wirtschaftlichen Aufschwung in den U.S.A. gegeben. Die positiv verlaufende wirtschaftliche Entwicklung der Vereinigten Staaten in den 50er und 60er Jahren veränderte sich danach grundlegend. Dieser wirtschaftliche Umschwung hatte verschiedene Ursachen; in seiner Wirkung war er jedoch eindeutig. Ende der 70er Jahre war die wirtschaftspolitische Neuorientierung der Vereinigten Staaten vorprogrammiert. Die Ökonomen, die eine nachfrageorientierte Wirtschaftspolitik präferierten, verloren ihren Einfluß auf die aktuelle Wirtschaftspolitik. Am Ende dieser Entwicklung stand im Januar 1981 der Präsidentenwechsel vom Demokraten Jimmy Carter zum Republikaner Ronald Reagan, der die wirtschaftliche Veränderung auch politisch zum Ausdruck brachte. Mit der Präsidentschaft Ronald Reagans erhielten die angebotsorientierten Wirtschaftspolitiker, die sogenannten SupplySider, Einfluß im Rahmen der Reaganomics auf die Wirtschaftspolitik der Vereinigten Staaten.

Die Wirtschaftskrise in den 70er Jahren war gekennzeichnet durch:

- geringes Produktivitäts- und Wirtschaftswachstum

- hohe Inflations- und Arbeitslosenraten.

Die Ursachenforschung hinsichtlich der Gründe für die negative wirtschaftliche Entwicklung erweist sich als schwierig. ${ }^{5}$ Eine Vielzahl von Einflüssen, teils exogener aber auch nationaler Art, belasteten die amerikanische Volkswirtschaft in den 70er Jahren schwer. Hierzu zählten die Einflußfaktoren:

- Ölkrisen,

- Vietnam-Krieg,

- soziologische

und

- wirtschaftspolitische Veränderungen.

Abbildung 1 soll deutlich machen, da $\beta$ es sich bei den wirtschaftlichen, politischen und gesellschaftlichen Veränderungen, die sich in den Vereinigten Staaten ausgehend vom Ende der 60er Jahre hin zum ökonomischen Kurswechsel unter Ronald Reagan er

4 Vgl. Malabre, A. L., Ungehörte Propheten: eine Insider Geschichte der modernen Ökonomie. Stuttgart 1994. S.144-146.

5 Vgl. Kurz, R., Angebotsorientierte Wirtschaftspolitik in den USA: Grundlagen, Praxis und Konsequenzen, Tübingen 1993, S. 72. 


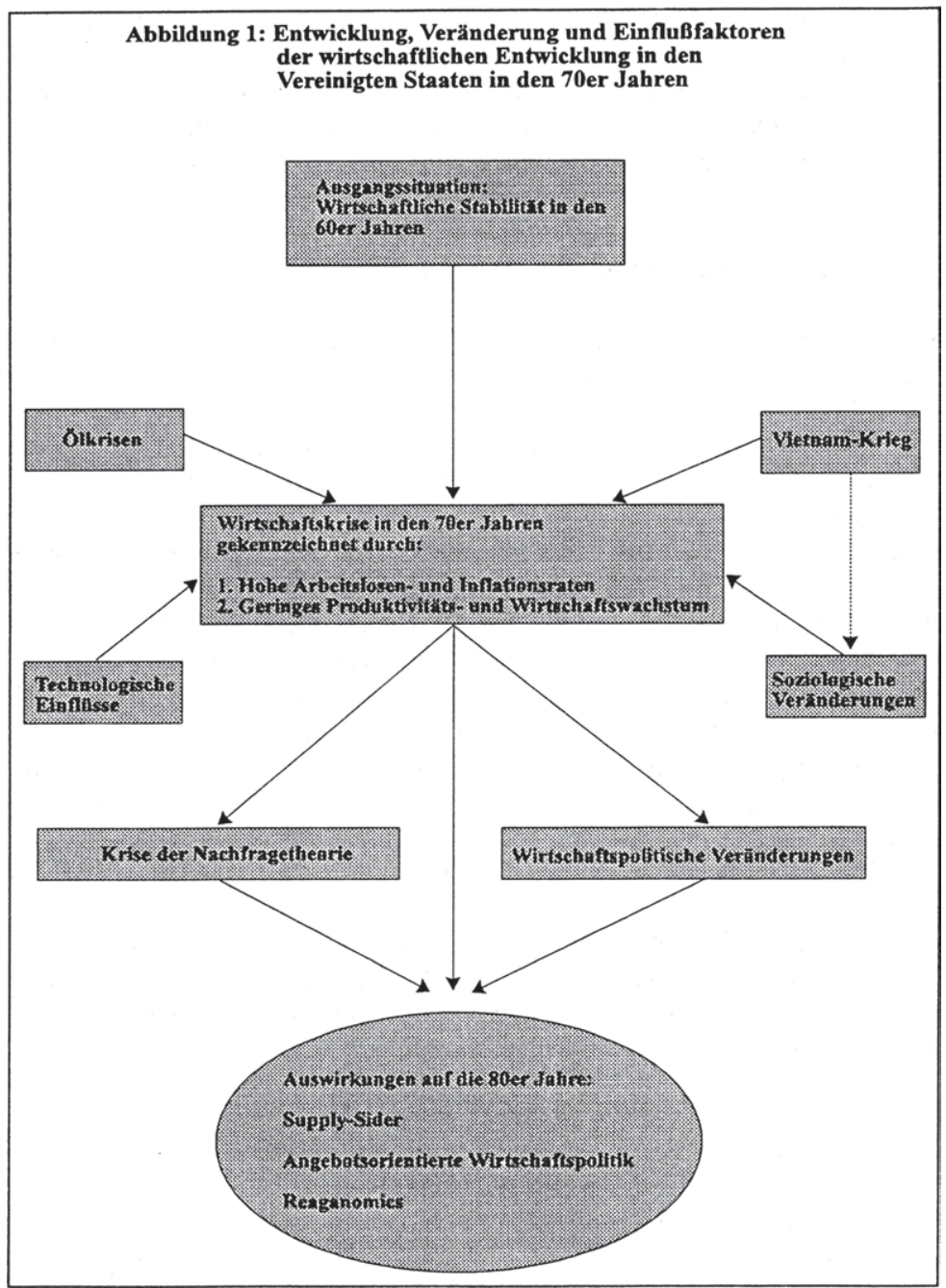

Quelle: eigene Darstellung.

gaben, um interdependente Ereignisse handelte. Das Zusammentreffen verschiedenster Faktoren erklärt den politischen und wirtschaftlichen Wandel in den U.S.A. zu Beginn der 80er Jahre. 
In den nachfolgenden Gliederungspunkten werden einzelne Komponenten der Abbildung 1 näher ausgeführt.

\subsection{Wirtschaftliche Auswirkungen des Vietnam-Krieges}

Die Niederlage der U.S.A. in Vietnam ließ das Selbstverständnis der amerikanischen Nation hinsichtlich ihrer militärischen und gesellschaftlichen Überlegenheit ins Wanken geraten. Die Auswirkungen des Vietnam-Krieges, der die U.S.A. von Mitte der 60er Jahre bis zur Flucht der letzten dort noch verbliebenen Amerikaner im April 1975 aus Saigon in seinen Bann zog, hatten viele Facetten, die die U.S.A. bis in die heutige Zeit beschäftigen. Aus ökonomischer Sicht drängt sich die Frage auf, ob in den wirtschaftlichen Auswirkungen des Vietnam-Krieges einer der Gründe für den wirtschaftspolitischen Wandel der 80er Jahre gesehen werden kann?

..The 1960s began with high promise for American life, but much of it was lost in Vietnam. The economic dimension is perhaps the least important, but serious enough. The War on Poverty petered out, the dream of the Great Society was not fulfilled, the grand macroeconomic design was discredited. The economy resumed an unstable course, with stubborn inflation and, in the 1970s, excessive unemployment too."6

Das Tobin-Zitat beschreibt in zutreffender Weise, daß die Eskalation des VietnamKrieges, die Mitte der 60er Jahre während der Präsidentschaft Lyndon B. Johnsons einsetzte, in jeder Hinsicht eine überaus starke Belastung für die U.S.A. darstellte. Die Verteidigungsausgaben gemessen in Anteilen am Bruttoinlandsprodukt stiegen nicht über das Niveau der Vorjahre (Tabelle 1).

Tabelle 1: Verteidigungsausgaben in Anteilen am Bruttoinlandsprodukt in den Vereinigten Staaten in den 60er Jahren (in \%)

\begin{tabular}{|l|c|c|c|c|c|c|c|c|c|c|}
\cline { 2 - 9 } \multicolumn{1}{c|}{} & \multicolumn{10}{c|}{ Jahr } \\
\cline { 2 - 9 } \multicolumn{1}{l|}{} & $\mathbf{6 0}$ & $\mathbf{6 1}$ & $\mathbf{6 2}$ & $\mathbf{6 3}$ & $\mathbf{6 4}$ & $\mathbf{6 5}$ & $\mathbf{6 6}$ & $\mathbf{6 7}$ & $\mathbf{6 8}$ & $\mathbf{6 9}$ \\
\hline $\begin{array}{l}\text { Verteidigungsausgaben in } \\
\text { Anteilen am Bruttoin- } \\
\text { landsprodukt } \\
\text { (in \%) }\end{array}$ & 8,8 & 9,0 & 9,1 & 8,5 & 7,8 & 7,3 & 8,1 & 9,0 & 8,9 & 8,2 \\
\hline
\end{tabular}

Quelle: National Income and Product Accounts of the United States, Volume 2, 1959-88, U.S. Department of Commerce, eigene Berechnungen.

6 Vgl. Tobin. J., Policies for Prosperity: Essays in a Keynesian Mode, The Political Economy of the 1960s. Brighton 1987, S. 437. 
Jedoch war der amerikanische Bundeshaushalt einer Doppelbelastung ausgesetzt. Präsident Johnson versuchte im Rahmen der sogenannten Konzeption der „Great Society" durch staatliche Programme zur Chancenförderung von Mitgliedern der unteren Sozialschichten eine Verminderung der Armut zu erreichen. ${ }^{7}$ Aus ökonomischer Sicht führte maßgeblich die Art und Weise der Finanzierung des Vietnam-Krieges und der „Great Society“ zu wirtschaftlichen Verwerfungen. Die gewählte Defizitfinanzierung des Krieges, die zunächst präferiert wurde - die meisten Ökonomen empfahlen eine Finanzierung über Steuererhöhungen - führte aufgrund der verstärkten Nachfrage zu einer Überhitzung der amerikanischen Wirtschaft, deren Kapazitätsgrenze auch ohne Kriegsfinanzierung schon erreicht war.

.The first acceleration of inflation, associated with the Vietnam War, was a classical demand-pull episode. President Johnson, contrary to the advice of his own economists, loaded his increased war spending on to an already fully employed economy without raising taxes, and the Federal Reserve were overaccommodative." 8

Das Budgetdefizit des Bundeshaushaltes stieg von 1,4 Milliarden Dollar im Jahr 1966 auf 12,7 Milliarden Dollar im darauffolgenden Jahr. Für die Jahre 1968 und 1969 beschloß die neue Administration unter Präsident Nixon eine spezielle Zusatzsteuer, die sogenannte „Vietnam war surtax“. Das Ergebnis war ein starker Anstieg der Einnahmen in den Jahren 1968 und 1969 verbunden mit einem Rückgang des Budgetdefizits, jedoch folgte ein neuerlicher starker Anstieg in den Jahren 1970 und 1971, als die Zusatzsteuer wieder auslief.; 1971 belief sich das Budgetdefizit auf 21,7 Milliarden Dollar. ${ }^{9}$

..Lyndon Johnsons bravely fought to combine guns and butter, to prevent his Great Society programmes from being sacrified for military spending. In a sense he was quite right: the society could well afford both. Vietnam War spending was never a large fraction of GNP, and at its height the total defence budget was smaller relative to the economy than in the 1950s. But in 1966 the economy could not finance both without an increase in taxes. Deficit spending overheated the economy and began the era of inflation and instability still afflicting us." 10

Die verstärkte staatliche Nachfrage aufgrund des Vietnam-Krieges führte zu den typischen Auswirkungen einer demand push inflation. Die Arbeitslosenrate lag im Jahr 1964 bei 5 Prozent. Von diesem Zeitpunkt an ging sie stark zurück, im Jahr 1969 waren

7 Vgl. Leber, M., „Reaganomics“ als wirtschaftspolitisches Vermächtnis, Dokumentation zur Wirtschaftskunde, Nr. 12, Dezember 1989, S. 3, 9.

8 Vgl. Tobin. J., Policies for Prosperity: Essays in a Keynesian Mode, Monetary Policy in an Uncertain World, Brighton 1987, S. 244.

$9 \mathrm{Vgl}$. Economic Report of the President, Februar 1994, S. 89.

$10 \mathrm{Vgl}$. Tobin, J., Policies for Prosperity: Essays in a Keynesian Mode, The Political Economy of the 1960s, Brighton 1987. S. 437. 
nur noch 3,4 Prozent ohne Arbeit. Im gleichen Zeitraum stieg die jährliche Inflation von 1,6 Prozent (1964) auf 5,5 Prozent (1969)11

..... the substantial excess demand during the Vietnam War period, which pushed the unemployment rate well below its full-employment level and gradually boosted inflation from 1.5 percent rate in the early 1960 s to 5 percent by the decade's end" 12

Ein Nebenprodukt dieser wirtschaftlichen Entwicklungen war eine pauschale Diskreditierung des Keynesianismus, weil die Nachfragesteuerung zu versagen schien. Die Ökonomen, die sich in ihren Vorstellungen an Keynes orientierten, wußten keine Antwort auf die Frage, wie Inflation und Arbeitslosigkeit gleichzeitig reduziert werden könnten. Aus der Ratlosigkeit der Keynesianer ergab sich eine Chance für die Angebotspolitik. ${ }^{13}$

Das angebliche oder tatsächliche Versagen der Theorie des Keynesianismus wurde von den Kritikern mit den hohen Inflations- und Arbeitslosenraten begründet. In den Auswirkungen auf die spätere Entwicklung spielt die Frage, ob diese Kritik im Hinblick auf die keynesianische Wirtschaftspolitik gerechtfertigt war, nur eine untergeordnete Rolle. Eine Finanzierung des Budgetdefizits in der Situation der Vollbeschäftigung über eine expansive Geldpolitik ist auch nicht im Sinn einer keynesianischen Wirtschaftspolitik. John Maynard Keynes hat bei einer derartigen wirtschaftlichen Konstellation niemals die Empfehlung ausgesprochen, diesen Prozeß durch staatliche Defizitprogramme weiter anzuheizen; das Gegenteil war der Fall.

..But what happens if a government. for whatever reason. continues to print money in large quantities even when the economy is at full employment? (The usual reason governments do this is to finance a budget deficit.) The answer is obvious: Once there are no longer idle factories and workers to draw into production. printing money will no longer raise production; it will simply raise prices.

Now Keynes always understood this. Keyne's first great book was not his famous „General Theory of Employment. Interest and Money“. but his „Tract on Monetary Reform“ (1923), an analysis of the hyperinflations that exploded in much of Europe after World War I. Thus Keynesian economics never called for expanding the economy without limit. Instead, it called for expanding the economy up to full employment, but no further. And it called for reining in the money supply when necessary in order to prevent inflation." 14

11 Vgl. Economic Report of the President, Februar 1994, Table B-62.-Changes in special consumer price indexes, 1958-93, S. 339.

$12 \mathrm{Vgl}$. Schultze. C. L.. Memos to the president: a guide through macroeconomics for the busy policymaker, The Brookings Institution, Washington, D. C. 1992, S. 169.

13 Vgl. Roberts, P. C.. Angebotsorientierte Wirtschaftspolitik, theoretische Grundlagen und politische Erfolge: Eine Bewertung der amerikanischen Erfahrungen in den achtziger Jahren, Zeitschrift für Wirtschaftspolitik, Heft 1, 1989, S. 7.

$14 \mathrm{Vgl}$. Krugman, P., Peddling Prosperity: eonomic sense and nonsense in the age of diminished expectations, New York-London 1994, S. 40. 
Die Wirtschaftspolitik der Vereinigten Staaten am Anfang der 70er Jahre wurde unter dem Deckmantel des Keynesianismus praktiziert. Die Defizitpolitik bei Vollbeschäftigung führte zu Inflation und verstärkte die Labilität der amerikanischen Volkswirtschaft bei unerwarteten exogenen Einflüssen.

Die Auswirkungen des Vietnam-Krieges waren somit ein wesentlicher Grund für den wirtschaftspolitischen Wandel der 80er Jahre. Vietnam sorgte dafür, daß der Wachstumsprozeß der amerikanischen Wirtschaft, der sich bis Mitte der 60er Jahre ohne größere externe Einflüsse ungestört entwickeln konnte, erheblich gestört wurde. Die Wirtschaftstheorie und Wirtschaftspolitik fanden kein adäquates ökonomisches Mittel, dieser Entwicklung in geeigneter Form entgegenzuwirken. Das wirtschaftliche Konzept der Nachfragesteuerung brachte unerwartete (erwartete) wirtschaftliche Ergebnisse: Inflation und Arbeitslosigkeit, die sogenannte Stagflation.

\subsubsection{Stagflation}

Bereits im letzten Quartal des Jahres 1969 sank das Bruttoinlandsprodukt mit einer jährlichen Rate von 1,3\%. Im darauffolgenden Jahr setzte sich diese negative wirtschaftliche Entwicklung fort. Die Statistiken belegen für dieses Jahr ein "reales Wachstum" von $0 \% .{ }^{15}$ In den Jahren 1971 und 1972 erholte sich die Wirtschaft nochmals, wobei die kurze wirtschaftliche Erholung nicht gänzlich ungetrübt war. Die Arbeitslosenquote, die in den Jahren 1968 und 1969 noch bei 3,5\% lag, stieg jeweils auf fast $6 \%$ in den Jahren 1971 und 1972. Die Inflation, gemessen am Konsumentenpreisindex, die bereits 1969 mit einer Rate von 5,5\% angestiegen war, blieb in den ersten Jahren des neuen Jahrzehnts auf einem für die Nachkriegsjahre der Vereinigten Staaten ungewöhnlich hohen Niveau. Im Jahr 1973 betrug die Inflationsrate im Jahresdurchschnitt $6,2 \% .{ }^{16}$

Im Zeitraum von 1968 bis 1973 lag die durchschnittliche jährliche Zunahme der Geldmenge M1 bei 6,2 Prozent, gegenüber 3,9 Prozent in den Jahren 1960 bis 1969. (Tabelle 2)

„Monetary policy was not tight enough to counteract the inflationary stimulus of the large Vietnam War deficits nor the overheated boom of 1972-73."17

Vietnamkrieg, Defizitpolitik, expansive Geldpolitik und Inflation führten dazu, daß im März 1973 das Fix-Kurssystem von Bretton Woods aus dem Jahr 1944, das die Bin-

$15 \mathrm{Vgl}$. National Income and Product Accounts of the United States, U.S. Department of Commerce. Economics and Statistics Administration Bureau of Economic Analysis, Volume 2, 1959-88, New York 1991, Table 8.1.-Percent Change From Preceding Period in Selected Series, S. $303 \mathrm{ff}$.

16 Vgl. Economic Report of the President, Februar 1994, Table B-62.-Changes in special consumer price indexes, 1958-93, S. 339.

$17 \mathrm{Vgl}$. Schultze, C. L., Memos to the president: a guide through macroeconomics for the busy policymaker, The Brookings Institution, Washington, D. C. 1992, S. 190. 
dung ausländischer Währungen über den Dollar an Gold in einem festen Austauschverhätnis festlegte, endgültig zusammenbrach. Im Jahr 1969 mußten noch 3,9251 DM im Jahresdurchschnitt für einen Dollar bezahlt werden, nach dem Zusammenbruch des Bretton Woods-System lag dieses Austauschverhältnis im März 1973 bei 2,8131 DM für einen Dollar; 1979 mußten im Durchschnitt nur noch 1,8343 DM für einen Dollar aufgebracht werden. ${ }^{18}$

Tabelle 2: Geldmengenwachstum MI in den Vereinigten Staaten, $1960-1979$, ausgewählte Zeiträume (in \%)

\begin{tabular}{|c|c|c|c|}
\cline { 2 - 4 } \multicolumn{1}{c|}{} & $\mathbf{1 9 6 0 - 1 9 6 9}$ & $\mathbf{1 9 6 8 - 1 9 7 3}$ & $\mathbf{1 9 7 0}-\mathbf{1 9 7 9}$ \\
\hline $\begin{array}{c}\text { Geldmengenwachstum } \\
\text { M1 }\end{array}$ & $+3,9 \%$ & $+6,2 \%$ & $+6,5 \%$ \\
\hline
\end{tabular}

1: durchschnittliche jährliche Veränderungsrate der Geldmenge MI

Quelle: Economic Report of the President, 1994, S. 347, eigene Berechnungen.

Ab dem Winter 1973 änderte sich endgültig das wirtschaftliche Szenario in den Vereinigten Staaten, Inflation und Arbeitslosigkeit sollten ein unerwartet hohes Ausmaß erreichen.

Im Nachhinein läßt sich die wirtschaftliche Lage der 70er Jahren in den U.S.A., insbesondere im Vergleich mit den 60er Jahren, als eine Dekade beschreiben, die von niedrigen Wachstumsraten des Sozialprodukts, hohen Inflationsraten, steigender Arbeitslosigkeit und sinkender Produktivität und gekennzeichnet war. (Tabelle 3)

Tabelle 3: Vergleich der wirtschaftlichen Entwicklung der 60er und 70er Jahrein den Vereinigten Staaten

\begin{tabular}{|l|c|c|}
\cline { 2 - 3 } \multicolumn{1}{c|}{} & 60er Jahre & 70er Jahre \\
\hline Bruttoinlandsprodukt $^{\mathbf{1}}$ & $4,1 \%$ & $2,9 \%$ \\
\hline Inflation $^{2}$ & $2,4 \%$ & $7,1 \%$ \\
\hline Arbeitslosigkeit $^{\mathbf{3}}$ & $4,8 \%$ & $6,2 \%$ \\
\hline Produktivität $^{\mathbf{4}}$ & $2,9 \%$ & $1,5 \%$ \\
\hline
\end{tabular}

1: durchschnittliche jährliche Veränderungsrate des Bruttoinlandsprodukts in Preisen von 1987

2: durchschnittliche jährliche Veränderungsrate des Konsumentenpreisindexes

3: durchschnittliche jährliche Arbeitslosenrate

4: durchschnittliche jährliche Veränderungsrate der Produktivität (Output per hour of all persons Business sector)

Quelle: Economic Report of the President, Februar 1994, S. 271,315,323 und 339, eigene Berechnungen.

18 Vgl. Economic Report of the President, Februar 1994, Table B-110.-Foreign exchange rates, 1969-93, S. 394. 


\subsection{3 Ölpreiskrisen}

Am 6.Oktober 1973, dem heiligen Versöhnungsfest der Juden, begann der vierte arabisch-israelische Krieg, der sogenannte Jom-Kippur Krieg. Dieses geschichtliche Ereignis war der Auslöser für die schwerste Rezession in den Industrieländern seit der Weltwirtschaftskrise. Der Krieg, der von Ägypten und Syrien begonnen wurde, endete am 22. Oktober 1973 mit einem Sieg Israels. Daraufhin beschlossen die arabischen Erdölausfuhrländer ihre Ölförderungen um $25 \%$ einzuschränken. Auf das arabische Ölembargo reagierte die Organisation der erdölexportierenden Länder (OPEC) in einer solidarischen Entscheidung mit ihren arabischen Mitgliedsstaaten mit einer Vervierfachung des Rohölpreises.

"The world won't be the same, after the Arab oil embargo in October 1973 and OPEC's quadrupling of oil prices." ..."In 1974 strong anti-inflationary measures in the United States and other major countries triggered or accentuated the worst postwar economic recession and lifted unemployment to rates unprecendented since the Great Depression." 19

Die Ölkrise ließ die Inflationsrate sehr stark ansteigen. Im Jahr 1974 stiegen die Konsumentenpreise mit einer Jahresrate von $11,0 \%$, der Produzentenpreisindex erhöhte sich sogar um 15,4\%.$^{20}$ Erstmals seit dem Korea-Krieg stiegen die Preise in den U.S.A. wieder mit einer zweistelligen Zuwachsrate.

Wer bis dahin an den Zusammenhang zwischen hoher Inflationsrate und geringer Arbeitslosenquote glaubte, mußte seine Einschätzung spätestens 1974 überdenken. Die sogenannte Phillips-Kurve - niedrige Inflationsraten gehen mit größeren Arbeitslosenquoten einher und vice versa - ließ sich in den 60er Jahren für die Vereinigten Staaten scheinbar annähernd ermitteln. Die nachfolgenden Jahre bewiesen, daß es keinen "trade off" zwischen Inflation und Arbeitslosenrate gibt, der ausschließlich auf diese zwei Einflußfaktoren zurückzuführen ist (Graphik 2).

Die Reihe der externen Schocks, die die Volkswirtschaft der U.S.A. schwer belasteten, setzte sich fort. Nach Vietnam und OPEC-Ölkrise folgte am Ende des Jahrzehnts die zweite Ölkrise. Im Zug der Revolution im Iran, 1978 bis 1980, und der Rückkehr des Ayatollah Khomeini aus dem Exil in Frankreich nach Teheran und den daraus resultierenden Lieferengpässen beim Rohöl verdreifachte sich der OPEC-Ölpreis ein weiteres Mal. In Folge des erneuten Preisschocks stieg die Inflation 1979 zum zweitenmal in diesem Jahrzehnt sprunghaft an. (Graphik 2) Diese Entwicklung verstärkte sich im darauffolgenden Jahr sogar noch. Dem Nachfrageschock Vietnam-Krieg, der die amerikanische Wirtschaft vom stetigen Wachstumskurs abbrachte, folgten zwei

19 Vgl. Tobin, J., Policies for Prosperity: Essays in a Keynesian Mode, Economic Stabilization Policies in the United States, Brighton 1987, S. 440.

$20 \mathrm{Vgl}$. Economic Report of the President, Februar 1994, Table B-62.-Changes in special consumer price indexes, 1958-93, Table B-67.-Changes in producer price indexes for finished goods. 1955 93, S. 339, S. 346. 
Preis- oder Angebotsschocks, Ölkrise I und II, die auf eine labile Wirtschaft trafen und folglich den Abwärtstrend verstärkten.

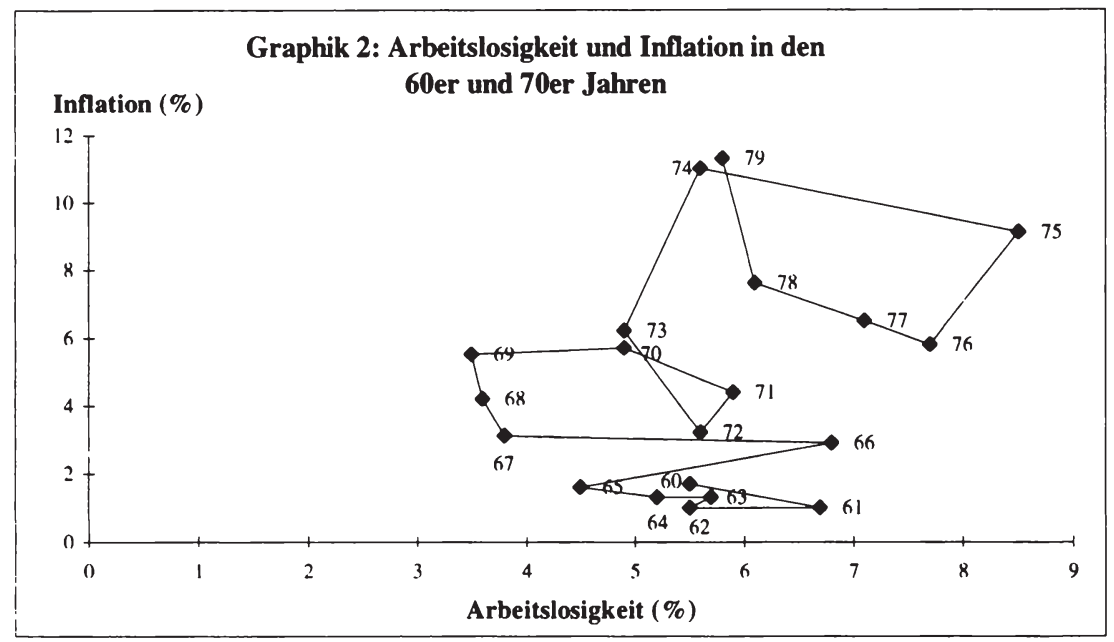

Quelle: Economic Report of the President, Februar 1994, S. 315, 339, eigene Darstellung.

..The two bursts of inflation in the 1970s were associated with extraordinary supply and price shocks. the first in 1973-74 by the Yom Kippur War, the oil embargo, and OPEC's fourfold increase in the dollar price of oil, the second in 1978-80 by the Iranian revolution, restriction of Middle East oil supplies, and a further tripling of the OPEC price. " 21

In den ersten Jahren nach der ersten Ölkrise wurde das Verlangsamen des Produktivitätswachstums in den U.S.A. auf den weltweiten Anstieg der Rohölpreise zurückgeführt. ${ }^{22}$ Diese Einschätzung für die Ursache der wirtschaftlichen Lage ließ sich jedoch nicht aufrecht erhalten. Das Ausmaß und die Dauer der Rezession waren zu ausgeprägt, als daß sie ausschließlich mit einem Ansteigen der Ölpreise hätten erklärt werden können. Das gleichzeitige Auftreten von hohen Inflationsraten und geringen Wachstumsraten des Sozialprodukts, die sogenannte Stagflation, traf die U.S.A. im Vergleich zu anderen Industrienationen, deren wirtschaftliche Entwicklung ebenso vom starken Anstieg der Ölpreise negativ beeinflußt wurde, besonders hart. ${ }^{23}$ Zwischen 1974 und 1978 sank

21 Vgl. Tobin, J., Policies for Prosperity: Essays in a Keynesian Mode, Monetary Policy in an Uncertain World, Brighton 1987, S. 244.

$22 \mathrm{Vgl}$. Krugman, P., Peddling Prosperity: eonomic sense and nonsense in the age of diminished expectations, New York-London 1994, S. 59.

23 Vgl. Boskin. M., Reagan and the Economy, The Success, Failures and Unfinished Agenda, San Francisco (CA) 1987, S 12. 
das reale Produktivitätswachstum pro Kopf gegen Null. Das stagnierende Wachstum war ein weltweites Phänomen. Tabelle 4 und die graphische Veranschaulichung (Graphik 3) zeigen jedoch, daß auch in anderen Industrienationen das Wachstum von starken zu moderaten Zuwächsen überging; in den U.S.A. hingegen stagnierte es völlig. ${ }^{24}$

Tabelle 4: Durchschnittliches jährliches Wachstum des Bruttosozialprodukts pro Beschäftigtem in den großen Industrienationen, 1964 - 1978, (in \%)

\begin{tabular}{|l|c|c|}
\cline { 2 - 3 } \multicolumn{1}{c|}{} & $\mathbf{1 9 6 4 - \mathbf { 1 9 7 3 }}$ & $\mathbf{1 9 7 3 - \mathbf { 1 9 7 8 }}$ \\
\hline U.S.A. & 1,8 & 0,1 \\
\hline Japan & 8,9 & 3,2 \\
\hline Deutschland & 4,7 & 3,0 \\
\hline Frankreich & 4,5 & 3,0 \\
\hline England & 3,2 & 0,8 \\
\hline Kanada & 2,4 & 0,6 \\
\hline Italien & 5,4 & 1,1 \\
\hline
\end{tabular}

Quelle: Economic Report of the President, 1979. S. 140.

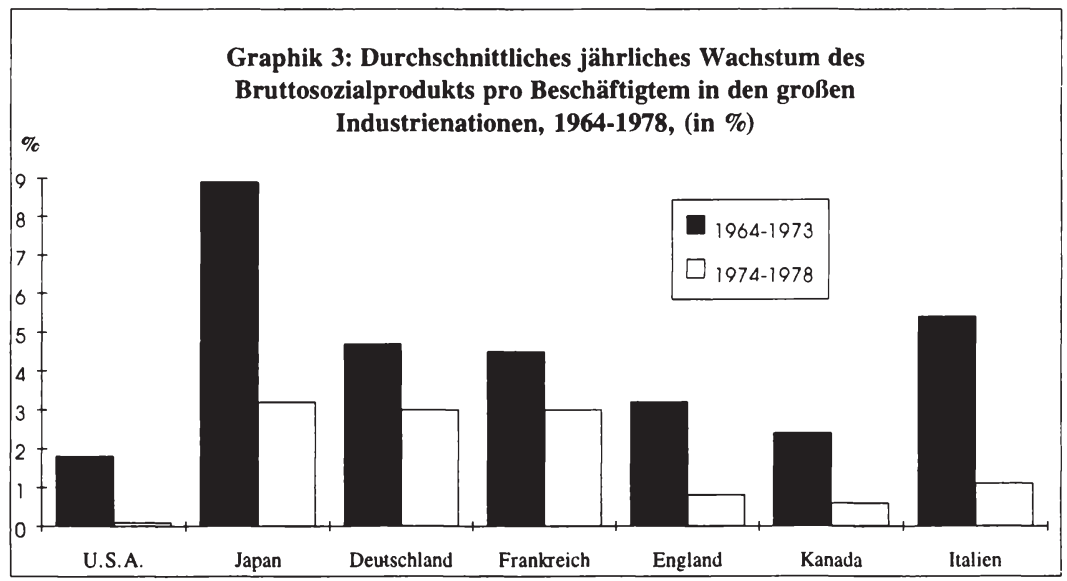

Quelle: Economic Report of the President. 1979, S. 68, eigene Darstellung.

24 Vgl. Hailstones, T. J., A Guide to Supply-side Economics, Richmond 1982, S. 71-76. 
Im internationalen Vergleich zeigt sich folgendes: Die Probleme in den Vereinigten Staaten müssen in den 70er Jahren wesentlich vielschichtiger gewesen sein, als daß sich der Erklärungsansatz für die Wirtschaftskrise in seiner Ursache ausschließlich auf den Ölpreisschock hätte reduzieren lassen können.

\subsubsection{Technologische und soziologische Veränderungen - Gründe für die Wirtschaftskrise}

Für die Wirtschaftskrise in den 70er Jahren lassen sich nach Krugman zwei weitere wesentliche Gründe anführen:

- technologische

und

- soziologische und Ursachen.25

Zu den technologischen Grïnden:

Das starke Produktivitätswachstum nach dem 2. Weltkrieg wurde getragen von einer Anzahl neuer Ideen und Techniken, die teilweise schon vor dem Krieg entwickelt wurden und nun Einzug in den Wirtschaftsprozeß hielten. Diese Entwicklung wurde begünstigt durch eine anhaltende Periode politischer und ökonomischer Stabilität. $\mathrm{Zu} \mathrm{Be}$ ginn der frühen 70er Jahre waren die Innovationen der Nachkriegsperiode feste Bestandteile des Wirtschafts- bzw. Produktionsprozesses geworden. Neue Technologien waren vorhanden, jedoch steckten sie in den Anfängen ihrer Entwicklung und wurden nur vereinzelt eingesetzt. ${ }^{26}$ Mit den 70er Jahre begann eine technologische und innovative Übergangsphase, die für die Produktivitätsentwicklung nur unzureichende Impulse aus neuen, entwickelten Technologien erhielt.

"The technological explanation of the productivity slowdown, then, asserts that by the early 1970s the set of technologies that had driven the postwar boom had been pretty much fully exploited. while the technologies that will eventually power another boom were not yet ready for prime time."27

Zu den soziologischen Gründen:

In den späten 60er und frühen 70er Jahren drängte die Generation aus der Zeit des Babybooms der ersten Nachkriegsjahre auf den amerikanischen Arbeitsmarkt. Die Zahl

$25 \mathrm{Vgl}$. Krugman. P., Peddling Prosperity: eonomic sense and nonsense in the age of diminished expectations. New York-London 1994, S. 59-65.

26 Vgl. Lamping, H.. Supply-Side Economics, Oldenburg 1988, S. 3.

$27 \mathrm{Vgl}$. Krugman. P.. Peddling Prosperity: eonomic sense and nonsense in the age of diminished expectations. New York-London 1994, S. 63. 
der zivilen Arbeitskräfte nahm sprunghaft zu. ${ }^{28}$ Im Jahre 1967 betrug das Arbeitskräftepotential 77,3 Millionen Menschen, bis 1974 stieg diese Zahl um 14,6 Millionen auf 91,9 Millionen. Die Zahl der Arbeitslosen erhöhte sich in diesem Zeitraum von 3 Millionen auf 5,2 Millionen Menschen. Für die Beschäftigungshöhe heißt dies, daß am Ende des Jahres 1974 etwa 12,4 Millionen Arbeitskräfte mehr beschäftigt waren als sieben Jahre zuvor. Die starke Zunahme des Arbeitskräfteangebots, die in den 70er Jahren anhalten sollte, verdeutlicht Tabelle 5 .

Tabelle 5: Durchschnittliche jährliche Zunahme des zivilen Arbeitskräfteangebots in den Vereinigten Staaten, 1960 - 1979, ausgewählte Zeiträume, (in \%)

\begin{tabular}{|l|c|c|c|}
\cline { 2 - 4 } \multicolumn{1}{c|}{} & $1960-1969$ & $1968-1973$ & $1970-1979$ \\
\hline $\begin{array}{l}\text { Zunahme des Arbeits- } \\
\text { kräfteangebots }\end{array}$ & $+1,7 \%$ & $+2,5 \%$ & $+2,7 \%$ \\
\hline
\end{tabular}

1: Arbeitskräfte, die 16 Jahre und älter sind und in zivilen Bereichen beschäftigt sind bzw. Beschäftigung suchen (Civilian labor force), durchschnittliche prozentuale Veränderung im Vorjahresvergleich für ausgewählte Zeiträume.

Quelle: Economic Report of the President, 1994, S. 306, eigene Berechnungen.

Die durchschnittliche jährliche Zunahme an Menschen, die auf den Arbeitsmarkt drängten, lag im Zeitraum von 1968 bis 1973 um 0,8 Prozentpunkte über dem Durchschnitt der 60er Jahre.

Aufgrund des Zuwachses des Arbeitskräftepotentials und der damit verbundenen wachsenden Beschäftigungszahl sank die Arbeitsproduktivität. ${ }^{29}$

Krugman ergänzt die soziologischen Gründe für die Produktivitätsverlangsamung um zwei nicht meßbare, aber dennoch wichtige Aspekte:

1) Teile der Gesellschaft, besonders die Gruppe, die in das Erwerbsleben trat, befanden sich Ende der 60er Jahre im Umbruch. Diese gesellschaftlichen Veränderungen waren gekennzeichnet durch einen Wandel der Lebenseinstellung bei der jungen Generation, die das dem Gesellschafts- und Wirtschaftssystem zugrundeliegende Wertesystem ablehnte. Der Kapitalismus und die Arbeit an sich wurden in Frage gestellt. Der sogenannten "Flower-Power-Generation" waren andere Dinge des Lebens wichtiger, als nur den Lebensunterhalt mit Arbeit zu sichern.

"Anyone who was touched, one way or another, by the cultural winds of the 1960s finds it plausible that social factors played a significant role in the 1970s slowdown. Surely productivity must have been

28 Das zivile Arbeitskäftepotential (Civilian labor force) umfaßt die Erwerbstätigen und die Arbeitslosen in allen Wirtschaftssektoren, ausgenommen sind dabei die Beschäftigten bei den Streitkräften.

29 Vgl. Tabelle 5. 
affected by a decade in which capitalism and work itself were denigrated, in which the mixture of high values and hypocrisy that holds society together seemed to lose its adhesive grip." 30

Diese gesellschaftliche Bewegung, die Ende der 60er Jahre die junge Generation in den U.S.A. erfaßte, war zu einem großen Teil in der gemeinschaftlichen Ablehnung gegenüber der Eskalation des Vietnam-Krieges begründet. Die Friedenssehnsucht auf dem Hintergrund der leidvollen Erfahrungen des Krieges erhöhte den Solidarisierungseffekt und trug maßgeblich zu einer geänderten Lebens- und Arbeitseinstellung bei.

2) Ende der 60er Jahre geriet das Bildungssystem in den Vereinigten Staaten in eine Krise. Das Ausbildungsniveau an den Schulen verringerte sich seit dieser Zeit in zunehmenden Maß. Diese Entwicklung traf weniger die Elite-Schulen, sondern vielmehr die Bildungseinrichtungen, die die Mehrzahl der Bevölkerung, insbesondere die Kinder der Mittelklasse, besuchte. Hinzu kam, daß in dieser Zeit in den U.S.A. die Unterschicht zu expandieren begann.

"So there is a plausible case to be made that social problems - the loss of economic drive among the children of the middle class, the declining standards of education, the rise of the underclass - played a significant role in the productivity slowdown." 31

Der sogenannte "Scholastic Aptidude Test", der die mathematischen und sprachlichen Kenntnisse der Studienanfänger in den U.S.A. prüft, weist seit Ende der 60er Jahre einen Rückgang bei den von den Studenten erzielten Testergebnissen aus. ${ }^{32}$

In den 70er Jahren spielte der einsetzende Rückgang des Bildungsniveaus hinsichtlich der Abschwächung des Produktivitätswachstums eine eher untergeordnete Rolle. Auf lange Sicht dürften die Probleme des amerikanischen Bildungssystems jedoch einen erheblichen negativen Einfluß auf den Rückgang der Produktivität und das Wirtschaftswachstum gehabt haben. Studien kommen zu dem Ergebnis, daß die durchschnittliche Qualität und Produktivität der Arbeitskräfte im Jahr 1990 erheblich schlechter war, als es die durchschnittliche Entwicklung der 50er und 60er Jahre, bei einer Beibehaltung des positiven Wachstumstrends für die nächsten Jahrzehnte, hätte vermuten lassen. ${ }^{33}$

$30 \mathrm{Vgl}$. Krugman, P., Peddling Prosperity: eonomic sense and nonsense in the age of diminished expectations, New York-London 1994, S. 63-64.

31 Ebenda, S. 64-65.

$32 \mathrm{Vgl}$. Schultze. C. L.. Memos to the president: a guide through macroeconomics for the busy policymaker. The Brookings Institution, Washington, D. C. 1992, S. 291.

33 Vgl. Bishop, J., ,Is the Test Score Decline Responsible for the Productivity Growth Decline?“住, American Economic Review, vol. 79, März 1989, S. 178-197. 


\subsection{Die Krise der Nachfragetheorie}

Bis zum Ende der 70er Jahre war die Wirtschaftspolitik der westlichen Industriestaaten von einer nachfrageorientierten ökonomischen Konzeption gekennzeichnet, die auf keynesianische Art der Stabilisierung ausgerichtet war. Die Ziele stetiges Wirtschaftswachstum, Preisniveaustabilität und geringe Arbeitslosigkeit standen dabei im Mittelpunkt der wirtschaftspolitischen Anstrengungen.

Die Finanzpolitik spielte eine entscheidende Rolle hinsichtlich einer störungsfreien gesamtwirtschaftlichen Entwicklung. Ihre Aufgabe bestand - gemäß der Keynesianischen Theorie der Nachfragesteuerung - darin, auf die Gesamtnachfrage einzuwirken. Im Fall einer unzureichenden gesamtwirtschaftlichen Nachfrage, die zur Vollauslastung des Produktionspotentials notwendig gewesen wäre, sollten zeitlich begrenzte Ausgabenprogramme der öffentlichen Hand die Nachfrageschwäche beheben. In den Vereinigten Staaten trat, wie beschrieben, Mitte der 70er Jahre eine wirtschaftliche Konstellation auf, vor der die bis dahin betriebene keynesianische Wirtschaftspolitik der antizyklischen Nachfragesteuerung zu versagen schien.

Gemäß der von Keynes entwickelten Theorie der Nachfragesteuerung sind Rezession und Arbeitslosigkeit eine Folge mangelnder Gesamtnachfrage. Die Aufgabe des Staates besteht darin, auf die Nachfrage so einzuwirken, daß diese stimuliert wird, bis das Niveau der Vollbeschäftigung wieder erreicht ist. Die Ausgabenseite des Haushaltsbudgets übernimmt in einer antizyklischen Variation der Staatsausgaben die Funktion, stimulierend bzw. dämpfend auf die konjunkturelle Entwicklung einzuwirken.

Graphik 4 zeigt, daß das Prinzip der antizyklischen Wirtschaftspolitik in den Vereinigten Staaten seit Anfang der 60er Jahre bis zum Ende der 70er Jahre weitgehendst praktiziert wurde.

Nach der ersten Ölkrise zeigte sich jedoch, daß die antizyklische Wirtschaftspolitik weder Arbeitslosigkeit noch Inflation nachhaltig reduzieren konnte. Zudem kam es zu einer Verlangsamung des Produktivitätswachstums in den Vereinigten Staaten, das im Vergleich zu anderen Industrienationen besonders stark ausfiel. (Tabelle 4, Graphik 3) Der Keynesianismus stand gleichsam als Synonym für alle Interventionsformen der Geld- und Fiskalpolitik in den Vereinigten Staaten in den 70er Jahren.

Dem gleichzeitigen Auftreten hoher Inflationsraten und zunehmender Arbeitslosenquoten konnten die keynesianisch ausgerichteten Ökonomen kein geeignetes ökonomisches Konzept entgegensetzen.

„Für das letzte Dezennium räumen jedoch selbst eingeschworene Keynesianer Schwächen und Mißerfolge ein. Gegenüber dem Phänomen der Stagflation und speziell vor einer Situation, in der Inflationsrate und Arbeitslosigkeit gleichzeitig zunehmen, muß das Konzept des Demand Management sein Versagen eingestehen. Genau diese Konstellation war aber während der siebziger Jahre weltweit immer wieder zu registrieren. Nicht zuletzt unter dem Eindruck dieser Entwicklung hat sich auch die Einschätzung der Wissenschaft deutlich gewandelt." 34

34 Vgl. Issing, O., Angebotsorientierte Wirtschaftspolitik, in: WiSt, Heft 10, Oktober 1982, S. 463. 


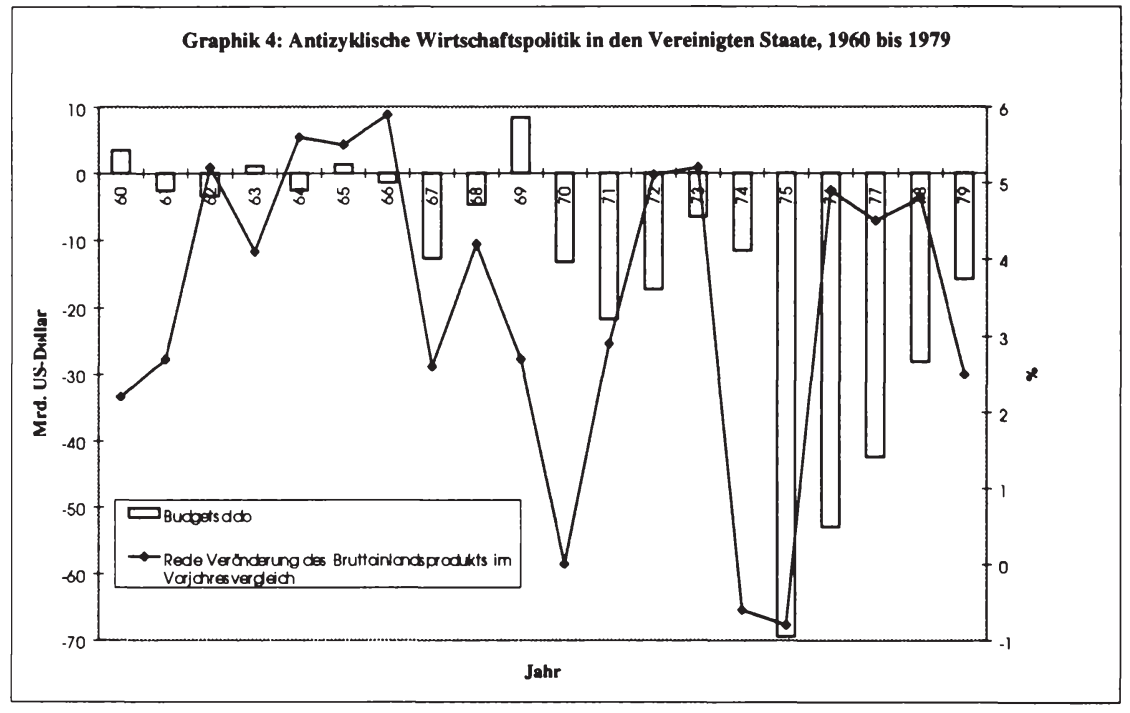

Quelle: National Income and Product Accounts of the United States, Volume 2, 1959-88, U.S. Department of Commerce, eigene Berechnungen.

Versagen angeblich alte Rezepte ist man empfänglicher für eine neue - erfolgversprechende - Therapie, weil

.... eine in den Wirtschaftswissenschaften etablierte Theorie immer dann in der Gefahr steht, verdrängt zu werden, wenn sie ein ökonomisches Problem zu erklären und zu lösen hat, das die bislang auf sie abgebildete Theorie nicht in ihrem Anwendungsbereich enthält." 35

\subsection{Wirtschaftspolitische Veränderungen}

Beim Keynesianismus, der begünstigt durch die Weltwirtschaftskrise nach dem 2. Weltkrieg Einzug in die Wirtschaftspolitik der Industriestaaten hielt, handelte es sich um eine "Revolution" im Rahmen der Wirtschaftstheorie. ${ }^{36}$

Mitte der 70er Jahre setzte in den Vereinigten Staaten jedoch eine „Neuorientierung“ der Wirtschaftspolitik ein. Sicherlich haben der Keynesianismus und die Supply-Side Policy eines gemeinsam: ihr anfänglicher Erfolg wurde begünstigt durch eine bestehen-

35 Vgl. Ehring, D.. Keynes. die Globalsteuerung und die Stabilisierungspolitik in der Bundesrepublik Deutschland, Frankfurt am Main 1989, S. 4-5.

$36 \mathrm{Vgl}$. Landmann, O.. Keynes in der heutigen Wirtschaftstheorie. in: Bombach et al. (Hrsg.): Keynesianismus I, Berlin-Heidelberg-New York 1981, S. $137 \mathrm{ff}$. 
de wirtschaftliche Krisensituation. Die Wirtschaftskrise in den 70er Jahren und die damit verbundene Kritik am Demand-Management waren der Auslöser für einen grundlegenden Wandel der amerikanischen Wirtschaftspolitik.

In den U.S.A. entwickelte sich eine konservative Strategie, die unzureichende Anreizmechanismen für die Wirtschaftskrise verantwortlich machte. ${ }^{37}$ Das Hauptaugenmerk lag dabei auf einer Verbesserung der Angebotsbedingungen, die staatliche Nachfragepolitik spielte dabei keine Rolle; sie wurde sogar für überaus schädlich erachtet. Diese Auffassung wurde zum politischen Dogma in den Vereinigten Staaten. Die Schuld für das schwache wirtschaftliche Wachstum wurde der Regierung gegeben. Die Angebotstheoretiker, die sogenannten Supply-Sider, betraten Mitte der 70er Jahre die politische Plattform in den Vereinigten Staaten.

Bartlett, einer der führenden Supply-Sider, vertritt in seinem Buch „Reaganomics“ die Auffassung, daß während der Rezession 1974 bis 1975 die keynesianisch ausgerichtete Wirtschaftspolitik gleichsam zu Grabe getragen wurde.

„In a real sense, Keynesian economics died during the recessions of 1974-75،“38

Die Frage, die danach in den Vereinigten Staaten diskutiert wurde, war: Welche „neue" Wirtschaftspolitik nimmt den Platz des Keynesianismus ein?39

Kristol, ein weiterer Supply-Sider, war einer der ersten, der diese Frage stellte und auch gleich eine Antwort parat hatte. In seinem Artikel für das Wall Street Journal im Mai 1977 schrieb er hierzu:

.In response to this crisis in the theory of economic policy, a „new" economics is beginning to emerge....Its focus is on economic growth, rather than on economic equilibrium or disequilibrium, and it sees such growth arising from a free response (e.g., investment, hard work, etc.) to the economic incentives of a free market.

... This new economics is sometimes described, rather cumbersomely, as „supply-side fiscal policy".... It arises in opposition to the Keynesian notion that an increase in demand, by itself, will increase supply and therefore accelerate economic growth. The "new“ economics asserts that an increase in demand, where the natural incentives to economic growth are stifled, will result simply in inflation. It is only an increase in productivity, which converts latent into actual demand by bringing commodities (old and new) to market at prices people can afford, that generates economic growth." 40

Den Anhängern der angebotsorientierten Wirtschaftspolitik gelang es in der öffentlichen Auseinandersetzung mit den Keynesianern, den Hauptunterschied an nur einem Element der Wirtschaftspolitik, das jedoch für alle Wirtschaftsakteure von besonderem Interesse ist, der Steuerpolitik, zu verdeutlichen. Zunächst setzte die Kritik der SupplySider an der keynesianischen Steuerpolitik an.

37 Vgl. Wendisch, P., Supply-Side Policy, Theoretische Grundlagen und prozeßpolitische Konsistenz, Frankfurt am Main 1984, S. 19.

$38 \mathrm{Vgl}$. Bartlett, B. R., Reaganomics: supply-side economics in action, Westport 1981, S. 3.

39 Ebenda.

40 Vgl. Kristol, I., Toward a 'New’ Economics?, Wall Street Journal, 9. Mai 1977. 
Sie argumentierten, daß für den Keynesianismus im Rahmen der Steuerpolitik nur das Volumen der Steuererhöhung bzw. -senkung entscheidend sei, da die aggregierte Nachfrage alleinig vom Gesamtvolumen der Steuerbelastung bestimmt werde. Aus diesem Grund sei es für die Keynesianer unwesentlich, ob die Steuern gesenkt oder die Staatsausgaben in gleichem Ausmaß erhöht werden, die Wirkung auf die Gesamtnachfrage ist in beiden Fällen identisch.

Dieser Einschätzung der keynesianischen Steuerpolitik stellten sie ihre Auffassung von einer angebotsorientierten Steuerpolitik gegenüber.

Die Steuerpolitik der Supply-Side Policy trägt folgende Grundzüge:

- Steuern haben die Funktion, einen maximalen Impuls auf die Höhe der Investitionen, das Sparaufkommen und das Arbeitsangebot auszuüben.

- Steuersenkungen nach keynesianischen Vorstellungen, die mit einer erhöhten Staatsverschuldung einhergehen, sind unsinnig, weil sie zu einer Verdrängung der privaten Investitionen (crowding out) führen. Die Finanzierung des Staatsdefizits über den Kapitalmarkt verursacht höhere Zinsen, die wiederum bewirken, daß die privaten Investitionen durch das höhere Zinsniveau verdrängt werden.

- Aus diesen beiden Grundsätzen ergeben sich für die Supply-Sider die Konsequenzen:

- Senkung des persönlichen Grenzsteuersatzes (marginaler Steuersatz), weil dadurch der Anreiz zu mehr Arbeit (weniger Freizeit), mehr Sparen (weniger Konsum) und mehr Investitionen seitens der Steuerpolitik für den privaten Sektor gegeben wird. Der marginale Steuersatz - oft auch als Grenzsteuersatz bezeichnet - entspricht dem Verhältnis der marginalen Änderung der Steuereinnahmen (Steuerbetrages) bei einer, im strikten Sinne, infinitesimal kleinen Änderung der Bemessungsgrundlage. Der Steuersatz, der für den letzten verdienten Dollar zu entrichten war, rückte in den Mittelpunkt der steuerpolitischen Diskussion. ${ }^{41}$ Die Supply-Sider waren der Auffassung, da $\beta$ insbesondere die hohen Grenzsteuersätze in den Vereinigten Staaten die Entfaltung jeder zusätzlichen wirtschaftlichen Aktivität behinderten. Besonders die Bezieher hoher Einkommen wurden durch hohe Grenzsteuersätze stark belastet (Tabelle 6).

- Senkung der Unternehmenssteuern, weil dadurch aufgrund des größeren Ertrags aus der Investition (Return on Investment) zum einen die Kapitalbildung für weitere Investitionen als auch der Anreiz für zusätzliche Investitionen gefördert werden.

Auf der Grundlage dieser Annahmen machten die Supply-Sider die Wirtschaftspolitik, insbesondere die Steuerpolitik, für die wirtschaftliche Misere in den Vereinigten Staaten verantwortlich. Die hohe Steuerbelastung hat nach ihrer Einschätzung zu unzureichenden Investitionen bei niedriger Sparquote und einer hohen Staatsquote geführt.

Die Entwicklung dieser Größen in den 70er Jahren im Gegensatz zu den 60er Jahren stütze ihre These (Tabelle 7).

41 Barro, R. J., Sahasakul, C.. Measuring the Average Marginal Tax Rate from the Individual Income Tax, Journal of Business, vol. 56, no. 4, 1983, S. 419-452. 
Tabelle 6: $\quad$ Grenzsteuersätze der Einkommensteuer in Abhängigkeit der Einkommenshöhe für einen Vier-Personen Haushalt in den Vereinigten Staaten, ausgewählte Zeiträume, (in \%)l

\begin{tabular}{|l|c|c|c|}
\cline { 2 - 4 } & \multicolumn{3}{c|}{ Familieneinkommen } \\
\cline { 2 - 4 } & $\begin{array}{c}\text { Haushalt mit } \\
\text { halbem } \\
\text { Durchschnittsein- } \\
\text { kommen }\end{array}$ & $\begin{array}{c}\text { Haushalt mit } \\
\text { Durchschnitts- } \\
\text { einkommen }\end{array}$ & $\begin{array}{c}\text { Haushalt mit } \\
\text { doppeltem } \\
\text { Durchschnitts- } \\
\text { einkommen }\end{array}$ \\
\hline $\mathbf{J a h r}$ & $20 \%$ & $20 \%$ & $22 \%$ \\
\hline 1960 & $14 \%$ & $17 \%$ & $22 \%$ \\
\hline 1970 & $15 \%$ & $20 \%$ & $26 \%$ \\
\hline 1975 & $17 \%$ & $22 \%$ & $32 \%$ \\
\hline 1980 & $18 \%$ & $24 \%$ & $43 \%$ \\
\hline
\end{tabular}

1: Ohne Sozialabgaben sowie ohne einzelstaatliche und kommunale Abgaben

Quelle: Department of the Treasury, Office of Tax Analysis, zitiert nach:

Palmer, J. L.,Sawhill, I. V., The Reagan Experiment: An Examination of Economic and Social Policies under the Reagan Administration, Washington, D.C. 1982, Table 4-3, S. 104. Kurz, R., Angebotsorientierte Wirtschaftspolitik in den USA: Grundlagen, Praxis und Konsequenzen, Tübingen 1993. S. 127.

Tabelle 7: Steuer-, Staats-, Konsum- und Investitionsquote in Anteilen am Bruttoinlandsprodukt in den Vereinigten Staaten, 1960 - 1979, ausgewählte Zeiträume, (in \%)

\begin{tabular}{|l|c|c|c|c|}
\cline { 2 - 5 } \multicolumn{1}{c|}{} & $\mathbf{1 9 6 0 - 1 9 6 9}$ & $\mathbf{1 9 6 8 - 1 9 7 3}$ & $\mathbf{1 9 7 4 - 1 9 7 9}$ & $\mathbf{1 9 7 0 - 1 9 7 9}$ \\
\hline Steuerquote1 $^{1}$ & $27,8 \%$ & $29,9 \%$ & $\mathbf{3 0 , 2} \%$ & $30,0 \%$ \\
\hline Staatsquote $^{2}$ & $19,1 \%$ & $20,4 \%$ & $\mathbf{2 1 , 6 \%}$ & $21,1 \%$ \\
\hline Konsumquote $^{3}$ & $60,8 \%$ & $62,7 \%$ & $\mathbf{6 4 , 6} \%$ & $64,1 \%$ \\
\hline Investitionsquote $^{\mathbf{4}}$ & $3,2 \%$ & $3,6 \%$ & $\mathbf{3 , 1} \%$ & $3,2 \%$ \\
\hline
\end{tabular}

1: Gesamtsteuerbelastungen (Einkommensteuern, Unternehmenssteuern, indirekte Steuern und Sozialabgaben) in Anteilen am Bruttoinlandsprodukt

2: Staatsausgaben des Bundes in Anteilen am Bruttoinlandsprodukt

3: Konsumausgaben in Anteilen am Bruttoinlandsprodukt

4: Netto-Investitionen außerhalb des Wohnungsbaus in Preisen von 1987 in Anteilen am realen Bruttoinlandsprodukt

Quelle: National Income and Product Accounts of the United States, Volume 2. 1959-88. U.S. Department of Commerce, eigene Berechnungen. 
Aufgrund des schwachen Produktivitäts- und Wirtschaftswachstums und der scheinbaren Ratlosigkeit seitens der bestehenden Wirtschaftstheorie, wie dieser Krise begegnet werden könnte, dauerte es nicht mehr lange, bis die Supply-Sider auch zunehmend Einfluß auf das politische Geschehen in den U.S.A. gewannen. Ende der 70er, Anfang der 80er Jahre, begann sich das politische Umfeld in den Vereinigten Staaten zu ihren Gunsten zu wandeln.

Mit dem Jahresbericht 1980 des Joint Economic Committee ${ }^{42}$ wird deutlich wie weit die Supply-Side Policy zu Beginn der 80er Jahre schon Einzug in die Wirtschaftspolitik der Vereinigten Staaten gehalten hatte:

..The 1980 annual report signals the start of a new era of economic thinking. The past has been dominated by economists who focused almost exclusively on the demand side of the economy and who, as a result, were trapped into believing that there is an inevitable tradeoff between unemployment and inflation. America does not have to fight inflation between during the 1980 s by periodically pulling up the drawbridge with recessions that doom millions of Americans to unemployment.

The Committee's 1980 report says that steady economic growth, created by productivity gains and accompained by a stable fiscal policy and a gradual reduction in the growth of the money supply over a period of years, can reduce inflation significantly during the 1980 s without increasing unemployment. To achieve this goal, the Committee recommends a comprehensive set of policies designed to enhance the productive side, the supply side of the economy....

The Committee recommends that fully one half of the next tax cut be directed to enhancing saving and investment in the economy. Traditionally, tax cuts have been viewed solely as countercyclical devices designed to shore up the demand side of economy. The Joint Economic Committee is now on record in support of the view that tax policy can and should be directed toward improving the productivity performance of the economy over the long term and need not to be enacted only to counter recession." 43

Es fehlte nur noch ein Präsident der Vereinigten Staaten, der die Vorstellungen der Supply-Sider konsequent in die wirtschaftspolitische Realität umsetzte.

\subsection{Resümee}

Das 1. Kapitel hat gezeigt, daß die Ursachen für die Wirtschaftskrise in den Vereinigten Staaten vielschichtig waren.

Vietnam-Krieg, Ölkrisen, soziologische- und technologische Veränderungen und letztlich dadurch ausgelöste wirtschaftspolitische Umbrüche schufen die Voraussetzung für die Verwirklichung der Supply-Side Policy.

Die wirtschaftlichen Indikatoren belegen, daß die U.S.A. zu Beginn der 80er Jahre in ihrer tiefsten wirtschaftlichen Krise seit dem 2.Weltkrieg steckte. (Tabelle 8)

42 Das Joint Economic Committee ist unter der gemeinsamen Aufsicht des Repräsentanten-Hauses und des Senats als legislativer Gegenpart des Council of Economic Advisers für die Analyse der gesamtwirtschaftlichen Entwicklung und für Empfehlungen zur wirtschaftlichen Globalsteuerung zuständig.

43 Vgl. Joint Economic Committee, Joint Economic Report, Senate Report No. 96-44, 96th Cong., 2d sess.. 1980, S. 1. 
Tabelle 8: Referenzgröß.ßen der wirtschaftlichen Entwicklung, ausgewählte Zeiträume, (in \%)

\begin{tabular}{|l|c|c|c|}
\cline { 2 - 4 } \multicolumn{1}{c|}{} & $1968-1973$ & $\mathbf{1 9 7 4 - 1 9 7 9}$ & $1970-1979$ \\
\hline Bruttoinlandsprodukt $^{\mathbf{1}}$ & $3,4 \%$ & $\mathbf{2 , 6} \%$ & $2,9 \%$ \\
\hline Produktivität $^{\mathbf{2}}$ & $2,3 \%$ & $\mathbf{0 , 8} \%$ & $1,5 \%$ \\
\hline Sparquote $^{\mathbf{3}}$ & $7,6 \%$ & $\mathbf{7 , 5} \%$ & $7,8 \%$ \\
\hline Investitionsquote $^{\mathbf{4}}$ & $3,6 \%$ & $\mathbf{3 , 1} \%$ & $3,2 \%$ \\
\hline Inflation $^{\mathbf{5}}$ & $4,9 \%$ & $\mathbf{8 , 6 \%}$ & $7,1 \%$ \\
\hline Arbeitslosigkeit $^{\mathbf{}}$ & $4,7 \%$ & $\mathbf{6 , 8} \%$ & $6,2 \%$ \\
\hline Budgetdefizit $^{\mathbf{}}$ & $0,8 \%$ & $\mathbf{2 , 0} \%$ & $1,7 \%$ \\
\hline
\end{tabular}

1: durchschnittliche jährliche Veränderungsrate des Bruttoinlandsprodukts in Preisen von 1987

2: durchschnittliche jährliche Veränderungsrate der Produktion pro Arbeitsstunde (Output per hour of all persons - Business sector)

3: durchschnittliches jährliches Sparen des privaten Sektors in Anteilen am verfügbaren Einkommen

4: durchschnittliche jährliche Nettoinvestitionen außerhalb des Wohnungsbaus in Preisen von 1987 in Anteilen am realen Bruttoinlandsprodukt

5: durchschnittlìche jährliche Veränderungsrate des Konsumentenpreisindexes

6: durchschnittliche jährliche Arbeitslosenrate

7: durchschnittliches jährliches Budgetdefizit (Budgetüberschuß) des Bundeshaushaltes in Anteilen am Bruttoinlandsprodukt

Quelle: National Income and Product Accounts of the United States, Volume 2, 1959-88, U.S. Department of Commerce, eigene Berechnungen.

In Anbetracht dieser negativen wirtschaftlichen Entwicklung, auf welche die bis dahin präferierte Wirtschaftspolitik keynesianischer Prägung keine Antwort wußte, war es nur allzu konsequent, daß der Keynesianismus verdrängt wurde.

Die sogenannte angebotsorientierte Wirtschaftspolitik (Supply-Side Policy) sah die Hauptursache für die Wirtschaftskrise in der zu hohen Steuerbelastung für die privaten Haushalte und den Unternehmenssektor. Die hohen Steuern verhinderten nach ihrer Einschätzung private Investitionen und bedingten somit die hohe Arbeitslosigkeit. Das Demand-Management hatte nach Auffassung der Angebotstheoretiker ausgedient, weil die staatliche Nachfragepolitik private Investitionen verhindert, Arbeitslosigkeit erhöht, die Inflation verstärkt und geringes Produktivitäts- und Wirtschaftswachstum auslöst.

Mit dem Amtsantritt von Ronald Reagan als 40. Präsident der Vereinigten Staaten im Januar 1981 war für die Supply-Side Policy endgültig der Weg frei. Es mußte sich nun zeigen, wie sich die Supply-Side Policy in der praktischen Ausgestaltung der Wirtschaftspolitik bewähren sollte.

Bevor im 3. Kapitel der Ausarbeitung dieser Frage eingehend nachgegangen wird, steht im 2. Kapitel zunächst die Theorie der Supply-Side Policy im Mittelpunkt der Analyse. 


\section{Die Theorie der Supply-Side Policy}

Die Theorie der Supply-Side Policy bildete das Fundament für den späteren Versuch, während der Präsidentschaft Ronald Reagans eine angebotsorientierte Wirtschaftspolitik in den Vereinigten Staaten zu verwirklichen. Die theoretischen Ansätze, das wird das zweite Kapitel zeigen, waren nicht grundlegend neu, aber in ihrer scheinbaren Klarheit für viele Politiker das lang erhoffte Mittel zur Überwindung der Wirtschaftskrise, wenigstens schien dies zunächst so.

\subsection{Die Supply-Sider - Akteure des wirtschaftspolitischen Wandels}

Die Entstehungsgeschichte der Supply-Side Policy in den Vereinigten Staaten begann Anfang der 70er Jahre. Die Keimzelle für die angebotsorientierte Wirtschaftspolitik war zu diesem Zeitpunkt in der Redaktion des „The Wall Street Journal“, eine der renomiertesten Wirtschaftszeitungen, zu finden.

.Journalisten haben weit weniger Einfluß, als sie oder die Öffentlichkeit zu glauben geneigt sind. Von dieser Regel gibt es jedoch Ausnahmen. Deren bemerkenswerteste sind vielleicht bestimmte Vorkommnisse in der schwierigen Zeit vor Anbruch der Reaganomics, wie die angebotsorientierte Wirtschaftspolitik oft abschätzig etikettiert wird. die während eines Großteils der Präsidentschaft Ronald Reagans praktiziert wurde."I

Im Wall Street Journal wurde in den Leitartikeln nachhaltig dafür plädiert, daß die Steuern gesenkt werden müssen. Es wurde dabei die Auffassung vertreten, daß Steuersenkungen nicht unbedingt zu einem höheren Staatsdefizit führen müssen, sondern sie vielmehr bei richtigem Einsatz eine Stimulierung der Wirtschaft bewirken. ${ }^{2}$ Robert Bartley und Jude Wanniski, beide Journalisten und keine ausgebildeten Ökonomen, bildeten den journalistischen Kern der Supply-Sider. Bartley war seit 1972 für die Leitartikel des „Journal“verantwortlich und Wanniski war sein Mitarbeiter.

..Above all, the cutting edge of the the supply-side movement consisted of the group that Robert Bartley assembled to preach on the editorial page of the The Wall Street Journal." 3

1 Vgl. Malabre, A. L., Ungehörte Propheten: eine Insider Geschichte der modernen Ökonomie. Stuttgart 1994, S. 215.

2 Vgl. The Wall Street Journal, Review and Outlook. Leitartikel vom 4. August 1976.

3 Vgl. Krugmann, P.. Peddling Prosperity: economic sense and nonsense in the age of diminished expectations. New York-London 1994, S. 85. 
$\mathrm{Zu}$ diesem Duo gesellten sich die Ökonomen Arthur Laffer und Irving Kristol. Als Urvater für ihre Theorie nannten die Supply-Sider den Wirtschaftswissenschaftler Robert Mundell, der 1971 in dem Artikel „The Dollar and the Policy Mix: 1971“ eine restriktive Geldpolitik kombiniert mit Steuersenkungen gegen die stagflationäre wirtschaftliche Entwicklung in den Vereinigten Staaten empfahl. ${ }^{4}$

"The correct policy mix was a reduction in the rate of monetary expansion (perhaps best achieved by a credit ceiling) combined with a tax reduction. This would have stopped the inflation rate without causing a depression." 5

Arthur Laffer fühlte sich durch die Arbeit von Mundell und insbesondere von seinem Vorschlag einer Steuersenkung zu eigenen Überlegungen inspiriert.

In diesem Zusammenhang erweist sich das Buch von Malabre „Ungehörte Propheten“" als aufschlußreiche Quelle. Arthur Laffer soll von Mundells Argumenten tief beeindruckt gewesen sein:

„Das war wie eine Erleuchtung für mich, erzählte Laffer einem Journal-Interviewer. Solchermaßen erleuchtet, konstruierte Laffer eine Theorie, wonach eine Steuersenkung einen derart explosiven Wachstumsschub auslösen könnte, daß dadurch alle anfänglichen Steuereinbußen für das Finanzministerium mehr als wettgemacht würden"6

Laffer war der Überzeugung, daß weniger die allgemeine Steuerbelastung in den U.S.A. von Nachteil sei als vielmehr die Einflüsse immer höherer Steuersätze auf höhere Einkommen, die den Leistungsanreiz verringerten. Von einer Kappung der Grenzsteuersätze versprach er sich verbesserte Angebotsbedingungen für die Wirtschaft, der eine verstärkte Nachfrage folgen würde. ${ }^{7}$ Diese Überlegungen waren nicht ganz neu, wenn man sich in diesem Kontext an das sogenannte Saysche Theorem erinnert, das den Zusammenhang von Angebot und Nachfrage schon im 19. Jahrhundert herstellte. Die Supply-Sider haben auch nie einen Hehl daraus gemacht, daß sie ihre Theorie an die Erkenntnisse der klassischen Wirtschaftstheorie anlehnten. ${ }^{8}$

Interessant bei der Propagierung der Supply-Side Policy ist das Zusammenspiel von Wirtschaftsjournalismus, Wirtschaftswissenschaftlern und Politikern.

4 Vgl. Mundell, R. A., The Dollar and the Policy Mix: 1971, Essays in International Finance. International Finance Section, no. 85, May, Princeton 1971, S. 3-28.

5 Vgl. Mundell, R. A., The Dollar and the Policy Mix: 1971, in: Recent Developments in Macroeconomics, Phelps, E. S. (Hrsg.), S. 410.

6 Vgl. Malabre, A. L., Ungehörte Propheten: eine Insider Geschichte der modernen Ökonomie. Stuttgart 1994, S. 222 und The Wall Street Journal, New Economics, Leitartikel vom 8. Oktober 1981.

7 Vgl. The Wall Street Journal, Tax-Cut Theorists, Leitartikel vom 1. Dezember 1978.

8 Vgl. Kapitel 2.2. 
„Ohne die Plattform des Wall Street Journal bzw. ihrer Leitartikelseite hätte Laffers Ökonomie in Washington und bei den Wirtschaftsführern der Vereinigten Staaten wohl nie so große Unterstützung gefunden."

Im Jahr 1975 nahm der republikanische New Yorker Kongreßabgeordnete Jack Kemp einen Mitstreiter für die Verwirklichung der angebotsorientierten Wirtschaftspolitik und Freund Laffers, Paul Craig Roberts, in seinen Beraterstab auf. Jack Kemp war ein Anhänger der angebotsorientierten Ideen Laffers. Er trug maßgeblich dazu bei, daß die angebotsorientierte Wirtschaftspolitik zur wirtschaftspolitischen Maxime erhoben wurde.

Paul Craig Roberts unterstützte Kemp dabei, bereits 1977 einen Gesetzentwurf einzubringen, der eine allgemeine dreißigprozentige Senkung der Grenzsteuersätze vorsah. Bei Senator William Roth fand er dabei weitere politische Unterstützung. Schließlich einigte man sich auf einen Dreijahres-Stufenplan zur Einführung dieser Steuersenkung. In einem ersten Schritt beschloß der Kongreß im Oktober 1978 ein SteuersenkungsGeamtvolumen von 19,7 Milliarden Dollar; u.a. wurde der Höchststeuersatz für große Kapitalgesellschaften von $48 \%$ auf $46 \%$ reduziert, eine Senkung der Kapitalertragssteuer und eine Erhöhung des persönlichen Steuerfreibetrags festgelegt.

Eine größere steuerliche Entlastung wurde verhindert, weil der damalige Präsident Carter sein Veto ankündigte. Im Gegenzug lehnte der Kongreß die von Carter vorgelegten Steuerreformgestze ab, die darauf ausgerichtet waren, Steuerschlupflöcher zu schließen, ohne simultan die Steuersätze zu senken. ${ }^{10}$ Für Roberts hatte spätestens zu diesem Zeitpunkt „die Revolution der angebotsorientierten Wirtschaftspolitik“ begonnen. ${ }^{\prime \prime}$

In dem Buch „Der Triumph der Politik“ vertritt David Stockman, später BudgetDirektor unter Ronald Reagan und ebenfalls Anhänger der angebotsorientierten Wirtschaftspolitik, die Auffassung, daß spätestens im Herbst 1980 die amerikanische Politik von einer revolutionären Kettenreaktion ergriffen war. ${ }^{12}$ Gemeint war hiermit die Supply-Side Policy.

Jack Kemp hatte in Betracht gezogen, bei der Präsidentschaftswahl 1980 für die Partei der Republikaner zu kandidieren. Ende 1979 gab er diesen Plan jedoch auf, als ihm der aussichtsreichere Bewerber Ronald Reagan versicherte, daß er sich für niedrigere Steuern einsetzen würde und Kemp die wirtschaftspolitische Linie Reagans ent-

9 Vgl. Malabre, A. L., Ungehörte Propheten: eine Insider Geschichte der modernen Ökonomie. Stuttgart 1994, S. 227.

$10 \mathrm{Vgl}$. Roberts, P. C., Angebotsorientierte Wirtschaftspolitik, theoretische Grundlagen und politische Erfolge: Eine Bewertung der amerikanischen Erfahrungen in den achtziger Jahren, Zeitschrift für Wirtschaftspolitik. Heft 1, 1989, S. 5.

11 Ebenda.

12 Vgl. Stockman, D., Der Triumph der Politik, Die Krise der Reagan-Regierung und ihre Auswirkungen auf die Weltwirtschaft. München 1986, S. 61. 
scheidend mitgestalten könne. ${ }^{13}$ Stockman berichtet, daß Kemp ihm Anfang 1980 folgende Mitteilung machte:

„Es sei gelungen, Reagan zu bekehren. ... Kemp kam strahlend zu mir und brachte Jude Wanniski und Art Laffer mit. Jack erzählte, sie seien mehrere Tage mit Reagan zusammengewesen und hätten über den Goldstandard, die Steuersenkungen, die theoretischen Grundlagen der Angebotsorientierung, das Wirtschaftswachstum und über unser ganzes Programm gesprochen. Und Gouverneur Reagan habe begeistert zugestimmt." 14

Das wirtschaftspolitische Programm der späteren Reagan-Administration läßt den Schluß zu, daß dieser Bericht Stockmans über die zitierte Kemp-Äußerung wohl der Wahrheit entsprochen hat.

Die Entstehungsgeschichte und der anfängliche Erfolg bei der Verwirklichung der Supply-Side Policy wurden durch mehrere Faktoren begünstigt. In diesem Zusammenhang wurde im ersten Kapitel auf die Wirtschaftskrise in den 70er Jahren und das Versagen der bis dahin praktizierten Nachfragepolitik bei der Lösung der Wirtschaftskrise verwiesen. $\mathrm{Zu}$ diesen Faktoren, die die Ausgangsbasis für die Verwirklichung der Supply-Side Policy bildeten, kamen weitere Einflußfaktoren hinzu:

- Eine Wirtschaftstheorie, die in ihrer zugespitzten Kernaussage: „Steuern müssen gesenkt werden" eine breite öffentliche Zustimmung fand.

- Politische Akteure, die es glänzend verstanden, die schwierige wirtschaftliche Lage, in der sich die U.S.A. zu diesem Zeitpunkt befand, für die Durchsetzung ihrer Ideen der Supply-Side Policy zu nutzen.

- Ronald Reagan, der für die angebotsorientierte Wirtschaftspolitik gewonnen werden konnte und dem es als höchstem Repräsentant der Vereinigten Staaten, als deren 40. Präsident, in zwei Amtszeiten von 1981 bis 1988 möglich sein sollte, die angebotsorientierte Wirtschaftspoltik in Form der Reaganomics zu übernehmen und in die Praxis umzusetzen.

Die Abbildung 2 zeigt die wichtigsten Verteter der Supply-Side Policy. ${ }^{15}$

\subsection{Die theoretischen Grundlagen der Supply-Side Policy}

Üblicherweise bricht eine neue Theorie mit bisher scheinbar gültigen Regeln; dies gilt im Rahmen der Wirtschaftstheorie zum Beispiel für den Keynesianismus. In seinem 1936 erschienenen Buch „Allgemeine Theorie der Beschäftigung, des Zinses und des Geldes“ („The General Theory of Employment, Interest and Money“) hat John Maynard Keynes bewußt seine Ausführungen der bis dahin geltenden klassischen Wirtschaftstheorie entgegengestellt.

13 Ebenda, S. 62.

14 Ebenda, S. 63-64.

15 Kurz, R., Angebotsorientierte Wirtschaftspolitik in den USA, Grundlagen, Praxis und Konsequenzen, Tübingen 1993, S. 21. 


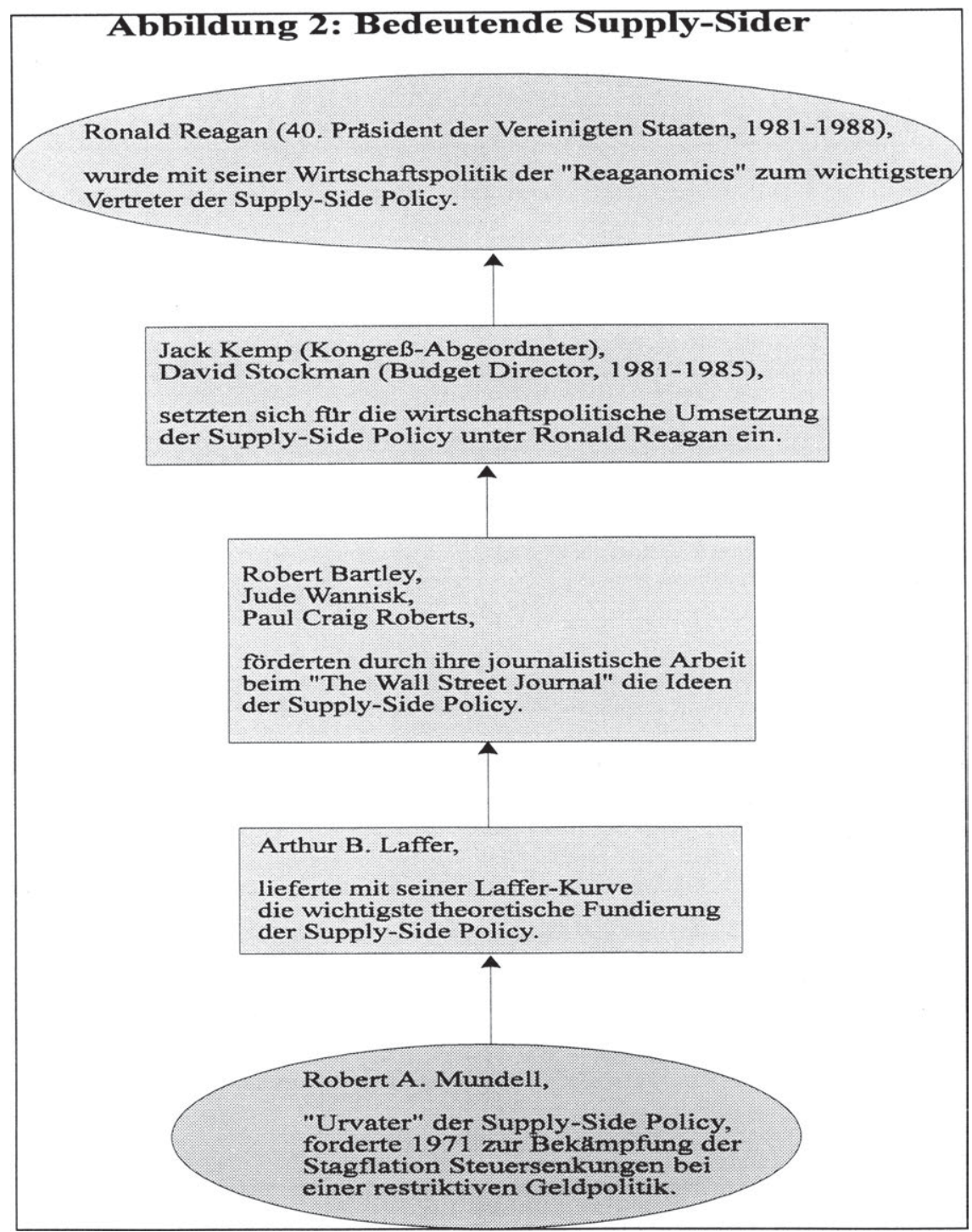

Quelle: eigene Darstellung. 
.Ich wähle diesen Titel, weil ich die Art meiner Beweisführung und Folgerungen jenen der klassischen Theorie über das Thema entgegenstellen will, jener Theorie, in deren Anschauungen ich erzogen worden bin, und welche heute, genau wie während der letzten hundert Jahre, das wirtschaftliche Denken und Handeln unserer regierenden und akademischen Kreise beherrscht. " 16

Keynes hat die klassische Wirtschaftstheorie zwar nicht zur Gänze verworfen, aber sie zu einem Spezialfall seiner eigenen erklärt.

„Ich werde darlegen, daß die Postulate der klassischen Theorie nur in einem Sonderfall, aber nicht im allgemeinen gültig sind, weil der Zustand, den sie voraussetzt, nur ein Grenzpunkt der möglichen Gleichgewichtslagen ist." 17

Die Theorie der Supply-Side Policy und deren Verfasser brachen mit dieser Tradition. Im Jahr 1982 sind die ersten beiden Bücher erschienen, „Supply-Side Economics in the 1980s" und "Supply-Side Economics: A Critical Appraisal", die in einer Zusammenstellung von Artikeln einen umfassenden Überblick über den Stand der Supply-Side Policy gaben. ${ }^{18}$ Es zeigten sich hier fundamentale Unterschiede zu den Anfängen des Keynesianismus:

- Die Theorie zur angebotsorientierten Wirtschaftspolitik kann nicht allein auf einen Verfasser zurückgeführt werden.

- Bei der Theorie handelt es sich nicht um einen grundlegend neuen Ansatz. In dieser Hinsicht erheben die Supply-Sider auch keinerlei Ansprüche. Ganz im Gegenteil, sie beziehen sich bewußt auf Aussagen der klassischen Wirtschaftstheorie.

Flassbeck geht über diese Einschätzung der Supply-Side Policy hinaus und vertritt die Auffassung, daß es sich bei der Angebotspolitik mehr um eine praktische als eine theoretische Revolution handle. ${ }^{19}$ Der Autor kommt in seinen weiteren Ausführungen zur Angebotspolitik zu dem Ergebnis, daß nicht der Monetarismus Keynes verdrängt hat, sondern faktisch die Angebotspolitik, die einen stabilitätstheoretischen Erklärungsansatz für die Wirtschaftskrise lieferte. ${ }^{20}$

Festzuhalten bleibt, daß sich die Exponenten der Supply-Side Policy explizit in ihren Ausführungen auf die klassische Wirtschaftstheorie bezogen.

.Supply-side economics does date from the time of Adam Smith. „The Wealth of Nations" talked about tax policy quite clearly and said that taxes should never be raised to a point where they discourage the industrousness of people." 21

16 Vgl. Keynes, J. M., Allgemeine Theorie der Beschäftigung, des Zinses und des Geldes, Berlin 1983, S. 1.

17 Ebenda.

$18 \mathrm{Vgl}$. Federal Reserve Bank of Atlanta (Hrsg.), Suppy-Side Economics in the 1980s, Atlanta 1982 und Fink, R. H. (Hrsg.), Supply-Side Economics: A Critical Appraisal, Maryland 1982.

19 Vgl. Flassbeck, H., Was ist Angebotspolitik?, in: Konjunkturpolitik, 28. Jahrg., Heft 2/3, 1982, S. 75 .

20 Ebenda, S. 79.

21 Vgl. Kemp, J., Supply-Side Economics: An American Renaissance, in: Suppy-Side Economics in the 1980s, 1982, S. 28. 
Keleher und Orzechowski, die eine umfassende Darstellung der historischen Ursprünge der Supply-Side Policy lieferten, gaben ihrem Beitrag für das Buch „SupplySide Economics: A Critical Appraisal“ bezeichnenderweise den Titel: „Supply-Side Fiscal Policy: An Historical Analysis of a Rejuvenated Idea". 22

Ohne Zweifel handelt es sich bei der Supply-Side Policy um wiederbelebte Ideen wesentlicher Elemente der klassischen Wirtschaftstheorie, die von den Supply-Sidern ergänzt wurden. Die angebotsorientierte Wirtschaftspolitik hat der klassischen Wirtschaftstheorie zu einer Renaissance verholfen. Es erscheint für das Verständnis der Bausteine der Angebotstheorie deshalb unerläßlich, sich die wichtigsten Bezugspunkte, die die Supply-Sider aus der Klassik aufgenommen haben, zu vergegenwärtigen.

Hierzu zählen:

- Der klassische Liberalismus

- Der klassische Preismechanismus

- Das Saysche Theorem

\subsubsection{Ursprünge der Supply'-Side Policy: Der klassische Liberalismus}

Im Jahr 1980 betrug in den U.S.A. der Anteil der Staatsausgaben am Bruttoinlandsprodukt 22,6\%. Das war, abgesehen vom Jahr 1975 mit fast $23 \%$, der bis zu diesem Zeitpunkt höchste Wert für die Staatsquote in den Vereinigten Staaten; 1960 lag diese noch bei knapp $19 \% .23$

Die Kritik am Keynesianismus fixierte sich seitens der Supply-Sider an der kontinuierlichen Ausweitung der Staatstätigkeit, zu deren Finanzierung die Steuerbelastungen für die Bürger - fast Zwangsläufig - erhöht werden mußten..$^{24}$

Für die Angebotstheoretiker vollzog sich mit dieser Entwicklung eine Abkehr von wichtigen Prinzipien der Marktwirtschaft, verbunden mit der Hinwendung zu einem Wohlfahrtsstaat, der den Menschen jegliches Lebensrisiko abzunehmen gedenkt. ${ }^{25}$ Hierin sahen sie die Hauptgefahr für die amerikanische Wirtschaft und Gesellschaft. Eine Politik, die die Risikobereitschaft der Wirtschaftssubjekte durch einen übergroßen Staatssektor zurückdrängt, handelt der Natur des Menschen zuwider. Die Supply-Sider vertraten die Auffassung, daß der Ansporn zur wirtschaftlichen Tätigkeit aus dem Eigeninteresse der Menschen resultiert. Stört der Staat durch sein eigenes starkes Engagement am Markt, finanziert über Steuererhöhungen, das individuelle Eigeninteresse

22 Vgl. Keleher, R. E., Orzechowski, W. P., Supply-Side Fiscal Policy: An Historical Analysis of a Rejuvenated Idea, in: Fink, R. H. (Hrsg.), Supply-Side Economics: A Critical Appraisal, Maryland 1982, S. 121-159.

23 Vgl. National Income and Product Accounts of the United States, Volume 2. 1959-88. Department of Commerce, New York 1991, eigene Berechnungen und Tabelle 7.

$24 \mathrm{Vgl}$. Tabelle 7.

25 Issing, O., Supply-Side Economics-Marginalien zu einem wirtschaftspolitischen Programm. in: Woll. A. (Hrsg.): Aktuelle Probleme der Wirtschaftspolitik. Schriften des Vereins für Socialpolitik, NF Bd. 130, Berlin 1983, S. 146. 
der anderen Wirtschaftsakteure, wird die gesamtwirtschaftliche Aktivität ein geringeres Niveau erreichen als bei einer die Eigeninteressen besser fördernden kleineren Staatsquote. 26

Diese Behauptung der Supply-Side Policy war nicht ganz neu.

Insbesondere im 18. Jahrhundert beschäftigten sich die Philosophen mit der Frage der Natur des Menschen. ${ }^{27}$ Das Buch „Wealth of Nations“ von Adam Smith aus dem Jahr 1776 faßte diese philosophischen Entwicklungen eindrucksvoll zusammmen und wurde zum Ausgangspunkt für die klassischen Wirtschaftswissenschaften. ${ }^{28}$ Mit Adam Smith setzte sich die Auffassung durch, daß sich der Wohlstand einer Gesellschaft in wirtschaftlichen Eigeninteressen (Eigennutzen) der Individuen gründet. Die vielen individuellen wirtschaftlichen Entscheidungen der einzelnen Wirtschaftssubjekte werden durch eine, wie Adam Smith sie bezeichnete, invisible hand so gesteuert, daß es zu einer Wohlstandsmaximierung kommt. ${ }^{29}$ Ein Staat, der einen hohen Anteil am Produktionsapparat beansprucht, stört in diesem Konnex den beschriebenen Prozeß und verhindert letztendlich ein höheres Wohlstandsniveau. Der materielle Anreiz bildet einen entscheidenden Bestimmungsfaktor für die Leistungsbereitschaft jedes Einzelnen. Ausgehend von diesem Anreiz muß es möglich sein, freie und selbständige Entscheidungen treffen zu können. Die Entscheidungen am Markt dürfen nicht durch einen dominanten Staat beeinträchtigt werden. Dieser Zusammenhang wurde zum zentralen Element des klassischen Liberalismus. ${ }^{30}$

Bis zur Weltwirtschaftskrise in den 30er Jahren dieses Jahrhunderts war dies die gängige Auffassung vom Wirtschaftsleben und der Rolle des Staates. Die Angebotstheoretiker des späten 20. Jahrhunderts sahen infolge des Keynesianismus die klassischen fundamentalen Gesetze des Wirtschaftens in Gefahr und wollten aus diesem Grund zurück zur ökonomischen Grundhaltung nach Adam Smith.

„So far from being new or revolutionary, supply-side economics is frankly reactionary. „Back to Adam Smith" can be fairly designated as its motto. Not, however, in the sense of returning to some purist version of laissez-faire - all supply-siders agree (as would have Adam Smith, author of The Theory of Moral Sentiments) that, when a society is sufficiently affluent to provide safety net for those unable to participate fully in the economy, we all have a moral obligation to see that such provision is made. „Back to Adam Smith“ has to be understood, rather, as „Back to The Wealth of Nations“ as the paradigm for economic reasoning... ." 31

26 Vgl. Gilder, G., Wealth and Poverty, New York 1981, S. 253.

27 Vgl. Schumpeter, J. A., Geschichte der ökonomischen Analyse, Göttingen 1965, S. 173-196.

28 Ebenda, S. 240-256.

29 Vgl. Hirschman, A. O., Interests, in: The New Palgrave: The World of Economics, New York 1991, S. 349-360.

30 Vgl. Reich, H.. Eigennutz und Kapitalismus: die Bedeutung des Gewinnstrebens im klassischen ökonomischen Denken, Berlin 1991, S. 50-73.

31 Vgl. Kristol, I., Ideology and Supply-side Economics, Commentary, April 1981, in: Economic Impact, No. 35, 1981/3, S. 32. 
Das Zitat macht deutlich, daß den Supply-Sidern kein Laissez-faire - Kapitalismus vorgehalten werden kann, der die Menschen, die in das soziale Abseits geraten sind, ihrem Schicksal überläßt. Die Gesellschaft (der Staat) hat die Verpflichtung, mit ihrem sozialen Netz diese Menschen aufzufangen. Das bedeutet eine moralische Verantwortung, welche die Gesellschaft zu tragen hat. In einem früheren Werk „The Theory of Moral Sentiments" aus dem Jahre 1759 führt Adam Smith aus, daß die marktmäßige Organisation der Wirtschaft nur funktionieren kann, wenn das Handeln der wirtschaftlichen Individuen von den sittlichen Prinzipien geleitet wird. ${ }^{32}$

Die Supply-Side Policy war insgesamt von einer konservativen Grundhaltung geprägt, die den innovatorischen Schwung der Vergangenheit mit seinem hohem Produktivitätszuwachs mit der Hinwendung zu alten Tugenden erreichen wollte. „Work, Family and Faith": Arbeit, Familie und Glaube sollten diese Entwicklung möglich machen. ${ }^{33}$

\subsubsection{Ursprïnge der Supply-Side Policy: Der klassische Preismechanismus}

Hinter dem von Adam Smith geprägten Begriff der unsichtbaren Hand (invisible hand) verbirgt sich der klassische marktwirtschaftliche Preisbildungsprozeß, der auf allen Märkten für ein Gleichgewicht zwischen angebotenen und nachgefragten Mengen sorgt. ${ }^{34}$

Die Wirtschaftssubjekte planen unter der Annahme der vollständigen Konkurrenz unabhängig voneinander und legen dabei ihre Angebots- und Nachfragemengen auf den Faktor- und Gütermärkten fest. Trotz verschiedener Einzelpläne kommt es zu einem Gleichgewicht zwischen angebotenen und nachgefragten Mengen auf den Märkten und folglich werden die Pläne der einzelnen Wirtschaftssubjekte erfüllt. Dieser Anpassungsprozeß wird durch die Wirksamkeit des Preismechanismus gewährleistet. Dieser bewirkt, daß sich die Produktionsstruktur, wenn auch mit zeitlicher Verzögerung, der Nachfragestruktur anpaßt. ${ }^{35}$

Die relativen Preise der Güter und Faktoren bilden die entscheidende Bestimmungsgröße für die Einzelpläne der Wirtschaftssubjekte, d.h. der Preis eines Gutes bzw. Faktors im Verhältnis zu dem Preis anderer alternativ angebotener Güter und Faktoren bestimmt die geplanten Angebots- und Nachfragemengen. ${ }^{36}$

32 Vgl. Reich. H., Eigennutz und Kapitalismus: die Bedeutung des Gewinnstrebens im klassischen ökonomischen Denken, Berlin 1991. S. 62-63.

33 Vgl. Gilder, G., Wealth and Poverty, New York 1981, S. 74.

34 Vgl. Wendisch, P.. Supply-Side Policy. Theoretische Grundlagen und prozeßpolitische Konsistenz. Frankfurt am Main 1984. S. 8.

35 Vgl. Felderer, B., Homburg, S., Makroökonomik und Neue Makroökonomik, Berlin 1987, S. 51.

36 Vgl. Wendisch, P., Supply-Side Policy, Theoretische Grundlagen und prozeßpolitische Konsistenz, Frankfurt am Main 1984, S. 8. 
Gerät ein Markt infolge einer Überschußnachfrage aus dem Gleichgewicht, so steigt der relative Preis des knapp gewordenen Gutes aufgrund des Preiswettbewerbs der Nachfrager. Dies führt einerseits zu einer Einschränkung der geplanten Nachfragemengen, da nicht alle Wirtschaftssubjekte bereit sind, den erhöhten Preis zu bezahlen, und andererseits zu einer Erhöhung der durchschnittlichen Einkommen derer, die an dem Produktionsprozeß des stärker begehrten Gutes beteiligt sind. Aufgrund der höheren Durchschnittseinkommen können mehr Produktionsfaktoren eingesetzt und somit das Angebot des Gutes gesteigert werden. Durch die Erhöhung des Angebots und des eintretenden Mengenwettbewerbs der Anbieter fallen wiederum die Preise. Das Angebot wird solange ausgedehnt, bis der Preis wieder auf sein ursprüngliches Niveau gefallen ist. Damit sinken auch die Einkommen der am Produktionsprozeß Beteiligten auf die ursprüngliche Höhe. Am Ende des Anpassungsprozesses ist der frühere Gleichgewichtszustand erreicht. ${ }^{37}$

Durch diesen Preismechanismus hat sich der Markt auf die veränderte Nachfrage ohne eine anhaltende Veränderung des Einkommens und der Preise eingestellt. Auslöser dieser Anpassungsprozesse sind die Konsumentenwünsche, der Markt paßt sich zeitverzögert an.

Wesentlich bei diesem Proze $\beta$ scheint, daß durch nichts anderes als dem Selbstinteresse und dem Eigennutz der Anbieter und Nachfrager, die versuchen, jeweils den größten Vorteil wahrzunehmen, die Anpassung bewirkt wird. So bringt das Eigeninteresse aller Beteiligten den Markt wieder ins Gleichgewicht, ohne daß irgendwelche Eingriffe von außen nötig wären. Staatliche Interventionen und Monopole würden diesen Anpassungsprozeß be- oder sogar verhindern, indem der Marktpreis künstlich über oder unter dem Gleichgewichtspreis gehalten würde. ${ }^{38}$

Dieser Anpassungsprozeß, der über die Preisbildung versucht, ein Gleichgewicht zwischen Angebot und Nachfrage zu schaffen, verläuft sowohl auf den Gütermärkten, als auch auf den Faktormärkten in der beschriebenen Weise; so auch auf dem Arbeitsmarkt.

Bei den „modernen“ Supply-Sidern standen, wie bereits erwähnt, die Steuern im Mittelpunkt ihrer Kritik. Steuern verändern im Zusammenhang mit dem klassischen Preisbildungsmechanismus die relativen Preise auf den einzelnen Märkten; marginale Steueränderungen sind als relative Preisänderungen zu interpretieren. Die Supply-Side Theorie begründete ihre Forderung nach einer Senkung der Steuersätze mit den positiven Auswirkungen auf die Angebotsseite. Steuersenkungen verändern die relativen Preise und erhöhen den Anreiz zur Änderung des Faktorangebotsverhaltens. Konkret heißt dies, daß mit einer Steuerpolitik, die auf eine Erhöhung der ökonomischen Anreize ausgerichetet ist, letztendlich die gesamtwirtschaftliche Produktion und folglich auch die Steuereinnahmen steigen. ${ }^{39}$

37 Ebenda, S. 8-11.

38 Vgl. Hansemeyer, K. H., Lehr- und Methodengeschichte, in: Kompendium der Volkswirtschaftslehre, Band 1, Göttingen 1967, S. 485-486.

$39 \mathrm{Vgl}$. Wendisch, P.. Supply-Side Policy, Theoretische Grundlagen und prozeßpolitische Konsi- 
,A supply-side cut in income and business taxes will probably result in some increase in the supply of labor, saving, investment, and hence, in aggregate supply. Because of this additional real growth, the tax base will increase and, hence. revenues will not fall in proportion to tax rates." 40

Die Wirkungszusammenhänge der Veränderung der relativen Preise finden sich im Rahmen der Supply-Side Theorie speziell im Wedge-Modell und in ihren Auswirkungen bei der Laffer-Kurve wieder. ${ }^{41}$

\subsubsection{Ursprünge der Supply-Side Policy: Das Saysche Theorem}

Eines der am häufigsten diskutierten, umstrittensten „Gesetze“ unter den Wirtschaftstheoretikern stellt der von Jean Baptiste Say im Jahr 1803 formulierte Zusammenhang zwischen Angebot und Nachfrage, das sogenannte Saysche- Theorem, dar. In seiner populärsten Form besagt das Saysche Theorem, daß dem gesamtwirtschaftlichen Angebot immer eine entsprechend große gesamtwirtschaftliche Nachfrage gegenübersteht bzw. das Angebot schaffe seine Nachfrage selbst.

..I trust this shows that it is not the abundance of money but the abundance of other products in general that facilitates sales. This is one of the most important truths of political economy." 42

Dieses Theorem bildete über einen längeren Zeitraum einen der zentralen Lehrsätze der klassischen Ökonomie. Keynes bestritt die Gültigkeit des Sayschen Theorems vehement und sah hierin auch die Notwendigkeit für seine Arbeit. ${ }^{43}$ In den Jahrzehnten des Keynesianismus war das Saysche Prinzip in den Hintergrund gedrängt worden. Erst mit der Krise des Keynesianismus erlangte das Saysche Gesetz wieder wirtschaftspolitische Relevanz, indem es als Argumentation dafür benutzt wurde, warum eine Hinwendung zu einer angebotsorientierten Wirtschaftspolitik nötig sei.

Nach dem Verständnis der Angebotsökonomen schafft die Produktion die Einkommen, aus denen die gesamtwirtschaftliche Nachfrage resultiert; werden alle Einkommen verausgabt, erzeugt sich das Angebot seine eigene Nachfrage. ${ }^{44}$

stenz, Frankfurt am Main 1984, S. 61-62.

40 Vgl. Keleher, R. E., Evidence Relating to Supply-Side Tax Policy, in: Fink. R. H. (Hrsg.). Supply-Side Economics: A Critical Appraisal, Maryland 1982, S. 272.

41 Vgl. hierzu Kapitel 2.2.5 und 2.2.6.

42 Vgl. Say, J. B., Traite d'economie politique, Paris 1803, zitiert nach: Baumol, W. J., Say`s (at least) Eight Laws, or What Say and James Mill may really have meant, in: Economica, 1977, Vol. 44. S. 148.

$43 \mathrm{Vgl}$. Keynes, J. M., The General Theory of Employment, Interest and Money, London 1936. S. 26.

$44 \mathrm{Vgl}$. Rahmann, B., Ist Angebotsökonomik eine instrumentelle Alternative zur keynesianischen Nachfrageökonomik?, in: Buttler. F., Kühl, J., Rahmann, B., Hrsg.. Staat und Beschäftigung, Angebots- und Nachfragepolitik in Theorie und Praxis, Beiträge zur Arbeitsmarkt- und Berufsfor- 
Die Produzenten bestimmen durch ihre produktive Tätigkeit die Höhe der Nachfrage. Daraus haben die Supply-Sider den Schluß gezogen, daß die Angebotsseite zur entscheidenden Determinante für die Entwicklung der Volkswirtschaft wird. Gelingt es, die ökonomischen Rahmenbedingungen so zu gestalten, daß ausreichend Anreize für die Angebotsseite zur Entfaltung ihrer wirtschaftlichen Aktivitäten bestehen, wird sich die Nachfrageseite im Sinne des Sayschen-Theorems entwickeln. Eine unzureichende gesamtwirtschaftliche Nachfrage kann aus diesem Grund niemals zum Problem einer Volkswirtschaft werden, sofern die Angebotsseite im Zentrum der wirtschaftlichen Bemühungen steht. ${ }^{45}$ In diesem Zusammenhang vollzieht sich auch die Einordnung des klassischen Preismechanismus, der die individuellen Wahlmöglichkeiten auf der Angebotsseite beeinflußt. Die Entscheidungen zwischen zusätzlichem Einkommen oder Freizeit und zukünftigen Investitionen oder sofortigem Konsum werden durch die marginalen Steuersätze beeinflußt. Je höher die Steuersätze auf das Einkommen um so geringer sind die Kosten der Freizeit und des Konsums.

„On the supply-side there are two important relative prices governing production. One price determines the choice between additional current income and leisure; the other determines the choice between additional future income (investment) and current consumption. Both prices are affected by the marginal tax rates. The higher the tax rates on earnings, the lower the cost of leisure and current consumption, in terms of foregone after-tax income." 46

Die Supply-Sider machten sich das Saysche-Gesetz in der Form zu Nutze, daß sie ihre Hauptbotschaft der Steuersenkungen mit dieser klassischen Erkenntnis der Wirtschaftswissenschaften untermauerten. Zudem machte es den Unterschied zwischen einer nachfrageorientierten und der von der Supply-Side Theorie geforderten angebotsorientierten Wirtschaftspolitik deutlich. ${ }^{47}$

Lamping kommt bei seiner Analyse der Supply-Side Economics zu der Einschätzung,

„daß die Angebotspolitik sich zwar auf zentrale Aussagen der klassischen Theorie stützt, diese aber zum Teil aus dem Gesamtzusammenhang gelöst und entsprechend den angebotspolitischen Überlegungen interpretiert hat. “48

schung, Institut für Arbeitsmarkt und Berufsforschung der Bundesanstalt für Arbeit, Nürnberg 1985., S. 87.

$45 \mathrm{Vgl}$. Kurz, R., Angebotsorientierte Wirtschaftspolitik in den USA: Grundlagen, Praxis und Konsequenzen, Tübingen 1993, S. 29.

46 Vgl. Roberts, P. C., The Breakdown of the Keynesian Model, in: Fink, R. H. (Hrsg.), Supply-Side Economics: A Critical Appraisal, Maryland 1982, S. 2.

47 Vgl. Gilder, G., Reichtum und Armut, Berlin 1981, S. 57.

48 Vgl. Lamping, H., Supply-Side Economics, Oldenburg 1988, S. 34. 


\subsubsection{Annahmen der klassischen Theorie - Fundament der Supply-Side Policy}

Die Kapitel 2.2.1, 2.2.2 und 2.2.3 haben gezeigt, daß sich die Theorie der Supply-Side Policy an den Kernaussagen der klassischen Wirtschaftstheorie anlehnt. Die grundlegenden Annahmen, die sich hinter diesen Aussagen verbergen, bilden hierbei im gleichen $\mathrm{Ma} ß$ das theoretische Fundament der Supply-Side Policy. Am Beispiel des Arbeitsmarkts wird dies besonders deutlich.

Bei einer klassischen Marktstruktur muß auf dem Arbeitsmarkt ein Gleichgewicht zwischen angebotener und nachgefragter Arbeit herrschen; ein Gleichgewicht bei Vollbeschäftigung. Dies kann nur dann der Fall sein, wenn ein Preis existiert, der zur Markträumung führt. Wie in Kapitel 2.2.2 analysiert, gilt auch für den Arbeitsmarkt bei der Angebotstheorie, da $\beta$ die relativen Preise das Gleichgewicht herstellen. Wird nun davon ausgegangen, daß für den Arbeitsmarkt das Güterpreisniveau gegeben ist, kann nur durch die Flexibilität der Nominallöhne das Gleichgewicht am Arbeitsmarkt gewährleistet bleiben. Eine Rigidität des Nominallohns, wie bei Keynes, ist nicht Bestandteil der Theorie der Supply-Side Policy. ${ }^{49}$ In der weiteren Konsequenz entwickelt sich hieraus die Schlußfolgerung, daß das Auftreten von Arbeitslosigkeit nur von freiwilliger Art sein kann, weil bei Flexibilität der Nominallöhne ein Gleichgewicht am Arbeitsmarkt immer erreicht werden muß.

Die Tendenz zum Gleichgewicht auf dem Arbeitsmarkt, sowie auf allen anderen Teilmärkten der Volkswirtschaft, wurde bei den Supply-Sidern auf der Grundlage der Annahmen der klassischen Wirtschaftstheorie erklärt. Die Auffassung der Angebotsökonomen, die den Staat für eine Beeinflussung der relativen Preise und damit für Marktungleichgewichte verantwortlich machten, leitete sich hieraus ab. Ein Unterschied zu „herkömmlichen“ Auseinandersetzungen im Rahmen der Wirtschaftstheorie war jedoch erkennbar: Bei den Supply-Sidern ging der Streit in diesen Fragen nicht so weit wie bei den Neoklassikern in der wissenschaftlichen Auseinandersetzung mit Keynes.

Hierfür sind drei Gründe auszumachen:

- Ein konsistentes Theoriegebäude liegt der Supply-Side Policy nicht zugrunde.

- Im Mittelpunkt der Supply-Side Theorie stand die praktische Umsetzung ihrer wirtschaftspolitischen Ideen, theoretische Elemente waren nur ein argumentatives Hilfsmittel.

- Die Konzentration der Supply-Sider ausschließlich auf die Angebotsseite der Volkswirtschaft war in der Geschichte der Wirtschaftswisenschaften kein neues Instrumentarium für die Bewältigung von Wirtschaftskrisen.

..Die Angebotsseite der Volkswirtschaft ist keineswegs die Neuentdeckung eines bisher unbekannten ökonomischen Funktionalzusammenhangs, der neue Erkenntnismöglichkeiten erschließt, sondern eine Wiederentdeckung bekannter und umstrittener Lehrsätze und Überzeugungen. Der Konflikt zwischen Angebots- und Nachfrageorientierung ist eigentlich mindestens so alt wie der Angriff Keynes' auf die für ihn ,orthodoxe klassische Theorie“." 50

49 Vgl. Wachtel, P., Makroökonomik: von der Theorie zur Praxis, Oldenburg 1994, S. 460-462.

$50 \mathrm{Vgl}$. Rahmann, B., Ist Angebotsökonomik eine instrumentelle Alternative zur keynesianischen 


\subsubsection{Die Laffer-Kurve}

Das wohl bekannteste theoretische Element der Supply-Side Policy ist die LafferKurve. Ihren Namen erhielt sie von dem Ökonomen Arthur B. Laffer, der Ende der 70er Jahre an der University of Southern California Volkswirtschaft lehrte. Die „Legendenbildung", die sich um die Entstehungsgeschichte der Laffer-Kurve rankt, will wissen, daß Laffer die Kurve erstmals 1974 in einem Restaurant in Washington auf einen Cocktailuntersetzer gezeichnet haben soll..$^{51}$

Die Laffer-Kurve wurde zur analytischen Basis für Ronald Reagans Steuerpolitik in den 80er Jahren. Nach seiner Amtseinführung als Präsident der Vereinigten Staaten präsentierte Ronald Reagan im Februar 1981 ein Steuerprogramm, das eine lineare Senkung des Einkommensteuersatzes vorsah..$^{52}$

\subsubsection{Anmerkungen zur Laffer-Kurve}

Laffer war der Auffassung, daß über eine Senkung der Steuerbelastung ein Anstieg der Steuereinnahmen in den Vereinigten Staaten erreicht werden könnte. Im nachfolgenden Zitat beschreibt Laffer den Verlauf der Kurve, und deutlich werden dabei auch die Annahmen, die dem Kuvenverlauf zugrundeliegen.

„If we plot tax rates on the horizontal axis and the total revenue on the vertical axis, all measured in real units, what we will find is that at zero tax rates we have zero revenue, and at 100 percent tax rates we also have zero revenue. If tax rates are increased from 0 percent to, say, 1 percent, total revenue will increase. While output may fall somewhat, there will be a positive tax rate applied to a positive amount of output. Likewise, if the government lowers the tax rate from 100 percent to 99 percent, revenue will also increase. At a 99 percent tax rate, there will be some low level of production and a high positive tax rate. Therefore, if tax rates are lowered from 100 percent, there is an increase in total revenue.... For each real revenue collected there are two different rates. ... Other receipts must rise if a rate is reduced. The expansion of activity will elicit a greater base upon which all other exchanged rates will obtain greater revenue. Government spending at all levels, will fall because of lowered unemployment, increased poverty, and thus less welfare. Likewise, government employees will require less in real wages because with lower tax rates, the same real wages will yield greater after tax wages, and so on. Finally, a cut in tax rates will yield greater savings in order to finance any deficit. Using a broader interpretation these tax rates and revenue positions should refer basically to the self-financing nature of tax rates changes." 53

Nachfrageökonomik?, in: Buttler, F., Kühl, J., Rahmann, B., Hrsg., Staat und Beschäftigung, Angebots- und Nachfragepolitik in Theorie und Praxis, Beiträge zur Arbeitsmarkt- und Berufsforschung, Institut für Arbeitsmarkt und Berufsforschung der Bundesanstalt für Arbeit, Nürnberg 1985., S. 68.

$51 \mathrm{Vgl}$. Fullerton, D., On the possibility of an inverse relationship between tax rates and government revenues, in: Journal of Public Economics 19, 1982, S. 3-22 und vgl. Malabre, A. L.. Ungehörte Propheten: eine Insider Geschichte der modernen Ökonomie, Stuttgart 1994, S. 226.

52 Vgl. Kapitel 3 und Issing, O., Die Laffer-Kurve, WiSt Heft 8, August 1981, S. 384-386.

53 Vgl. Laffer, A.B., An Equilibrium Rational Macroeconomic Framework, in: Kamrany, N./Day, R. (Hrsg.), Economic Issues of the Eighties. Baltimore 1979, S. 53-55. 
Bei der Analyse der Auswirkungen einer Steuersatzänderung auf die Höhe der Steuereinnahmen, das zeigen seine gewählten Beispiele, untersucht Laffer marginale Änderungen des Steuersatzes. Im Mittelpunkt der Theorie der Supply-Side Policy steht der Grenzsteuersatz (marginaler Steuersatz), der dem Verhältnis der marginalen Änderung des Steuerbetrages bei geringfügiger Änderung der Bemessungsgrundlage entspricht.

Die Änderung des Grenzsteuersatzes löst eine Änderung des Faktorangebotsverhaltens aus. Es entsteht bei einer Senkung des Grenzsteuersatzes ein verstärkter Anreiz zu vermehrtem Arbeiten, Sparen und Investieren. Dahinter verbirgt sich die Annahme, daß die Wirtschaftssubjekte ihre Entscheidungen an der Struktur der jeweiligen relativen Preise ausrichten. Die Wirtschaftssubjekte entscheiden zwischen Arbeit/Freizeit und Konsum/Sparen und Investieren in Abhängigkeit der jeweiligen Kosten der Entscheidungsalternative. Höhere Netto-Löhne, aufgrund eines niedrigeren Grenzsteuersatzes, führen zu einer relativen Verbilligung der Einkommenserzielung durch Arbeit zuungunsten der Höhe an Freizeit, und eine höhere Kapitalrendite nach Steuern verteuert den laufenden Konsum im Vergleich zu zukünftigen und momentanen Investitionen und Sparen. Marginale Steuersatzänderungen sind im Rahmen der Supply-Side Policy als relative Preisänderungen aufzufassen, die die Ressourcenverwendung der Wirtschaftssubjekte bestimmen und in Folge die Höhe der allgemeinen wirtschaftlichen Aktivität. ${ }^{54}$ Entscheidend für die wirtschaftliche Entwicklung sind dabei nicht etwaige Veränderungen auf der Nachfrageseite der Volkswirtschaft, sondern ausschließlich die Effekte der Steuerpolitik auf das gesamtwirtschaftliche Angebot.

.... changes in tax rates are changes in relative prices and thus affect choice, the allocation of ressources, and hence, real economic activity. According to this view, then, tax rate changes should be thought of as relative price changes and not as revenue or income changes; it is the change in relative prices and not the change in income or spending that matters for aggregate supply." 55

\subsubsection{Verlauf und Beschreibung der Laffer-Kurve}

Die graphische Veranschaulichung der Laffer-Kuve (Abbildung 3) ist an eine Veröffentlichung von Laffer aus dem Jahr 1979 angelehnt. ${ }^{56}$ Andere Veröffentlichungen zeigen die Laffer-Kurve in einer umgekehrten Bezeichnung für die Abszisse (Steuereinnahmen) bzw. Ordinate (Steuersatz). ${ }^{57}$

54 Vgl. Wendisch, P., Supply-Side Policy, Theoretische Grundlagen und prozeßpolitische Konsistenz, Frankfurt am Main 1984, S. 61.

$55 \mathrm{Vgl}$. Keleher, R. E., Evidence Relating to Supply-Side Tax Policy, in: Fink, R. H. (Hrsg.), Supply-Side Economics: A Critical Appraisal, Maryland 1982, S. 265.

56 Vgl. Laffer, A.B., An Equilibrium Rational Macroeconomic Framework, in: Kamrany, N., Day, R. (Hrsg.), Economic Issues of the Eighties, Baltimore 1979, S. 54.

$57 \mathrm{Vgl}$. Wendisch, P., Supply-Side Policy. Theoretische Grundlagen und prozeßpolitische Konsistenz, Frankfurt am Main 1984, S. 97. 


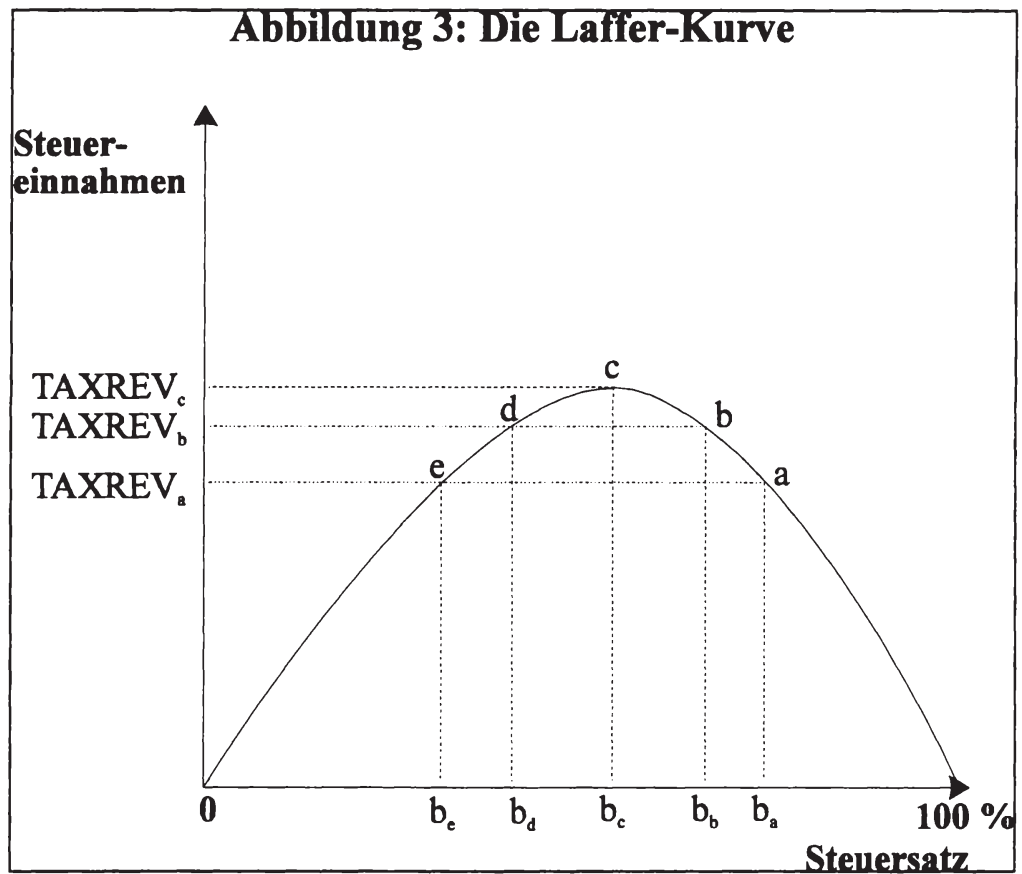

Quelle: Laffer, A.B., An Equilibrium Rational Macroeconomic Framework, in: Kamrany, N./Day, R. (Hrsg.), Economic Issues of the Eighties, Baltimore 1979, S. 54, eigene Darstellung.

Die Laffer-Kurve stellt den Zusammenhang zwischen Steuersatz und Steuereinnahmen dar. Die Steuereinnahmen (TAXREV) sind hierbei eine Funktion des Steuersatzes (b). Der Steuersatz kann Werte im Bereich zwischen 0\% und 100\% einnehmen. Der Staat hätte bei einem Steuersatz von $0 \%$ keine Steuereinnahmen, ebenso bei einem Steuersatz von $100 \%$, weil in diesem Extremfall wohl kein Wirtschaftssubjekt mehr bereit wäre, eine besteuerbare Leistung zu erbringen. In der graphischen Veranschaulichung zeigt sich, daß die Kurve zunächst - mit dem Verlauf einer Parabel vergleichbar bis zum Punkt $c$ degressiv ansteigt.

Im Punkt $c$ ist der kritische Steuersatz $\left(b_{c}\right)$ erreicht. Der Grenzertrag der Steuereinnahmen ist bei diesem Steuersatz gleich Null. Jenseits dieses Steuersatzes wird der Grenzertrag kleiner Null und die Laffer-Kurve fällt somit ab; was nichts anderes heißt, als daß die Steigung der Funktion negativ wird.

Im Bereich des sinkenden Teils der Laffer Kurve gilt - was zunächst paradox erscheinen mag - , daß mit einem kleineren Steuersatz höhere Steuereinnahmen erzielt werden. In diesem sogenannten prohibitiven Bereich (prohibitive range) wird dieses pa- 
radoxe Ergebnis durch das inverse Verhältnis zwischen Steuersatz und Steuereinnahmen erreicht.

Sinkt der Steuersatz von $b_{a}$ auf $b_{b}$ werden im Punkt $b$ höhere Steuereinnahmen $\left(T_{A X R E V}\right)$ als im Punkt a $\left(T_{A X R E V}\right.$ ) erreicht.

Die Abbildung der Laffer-Kurve zeigt auch, daß im normalen Bereich (normal range), $0 \leq b<b_{c}$ jeweils ein Steuersatz existiert, der zu den gleichen Steuereinnahmen führt, wie ein Steuersatz aus dem prohibitiven Bereich. Im Punkt e werden bei einem Steuersatz $b_{e}$ die gleichen Einnahmen wie im Punkt a bei einem Steuersatz $b_{a}$ erzielt; in den Punkten b und d kommt es ebenfalls bei einem unterschiedlichen Steuersatz zu identischen Steuereinnahmen.

Befindet sich eine Volkswirtschaft im prohibitiven Bereich gelingt es, sofern die Laffer-Kurve gilt, über Steuersenkungen höhere Steuereinnahmen zu erzielen. Voraussetzung dafür ist, daß eine höhere Leistungsbereitschaft der Wirtschaftssubjekte ausgelöst wird, weil aufgrund der geringeren steuerlichen Belastungen der ökonomische Anreiz für eine höhere Leistung geschaffen wurde. Die Volkswirtschaft wird auf einen höheren Wachstumspfad gelangen als vor der Steuersenkung.

Eine Reduktion der Steuerbelastung, die zu mehr Steuereinnahmen und Wirtschaftswachstum führt, ist von besonderem finanz- und steuerpolitischen Interesse. Die Regierung muß "nur" den optimalen Steuersatz finden, der sowohl die Steuereinnahmen maximiert als auch das gesamtwirtschaftliche Angebot festlegt, welches die Wirtschaftssubjekte nachfragen. Vielleicht ließ sich gerade aus diesem Grund Ronald Reagan für eine Steuerpolitik begeistern, die auf die Ergebnisse der Laffer-Kurve setzte.

Fullerton und Issing verweisen in ihren jeweiligen Beiträgen zur Laffer-Kurve darauf, daß die Laffer-These nicht neu ist. Jonathan Swift und Wilhelm Gerloff stellten vor Laffer bereits fest, daß eine Zollerhöhung die Zolleinnahmen nicht unbedingt vergrößern muß, so Swift, bzw., daß bei steigenden Steuersätzen die Steuerwiderstände, nach der Einschätzung von Gerloff, zunehmen würden..$^{58}$ Die Laffer-Kurve, der Zusammenhang zwischen Steuersatz und Steuereinnahmen, wurde bereits im Jahr 1844 von Jules Dupuit beschrieben.

..By thus gradually increasing the tax it will reach a level at which the yield is at a maximum... Beyond. the yield of tax diminishes... Lastly a tax (which is prohibitive) will yield nothing.. 59

\subsubsection{Tobin-Herleitung der Laffer-Kurve}

Die Laffer-Kurve (Abbildung 3) läßt sich mathematisch einfach beschreiben. Die Steuereinnahmen (TAXREV) sind definiert als eine Funktion des Steuersatzes (b). Der

$58 \mathrm{Vgl}$. Issing, O., Die Laffer-Kurve, WiSt Heft 8, August 1981, S. 385.

$59 \mathrm{Vgl}$. Dupuit, J., On the measurement of the utility of public works, 1844, zitiert nach: Fullerton. D.. On the possibility of an inverse relationship between tax rates and government revenues, in: Journal of Public Economics 19, 1982, S. 5. 
Steuersatz kann Werte zwischen $0 \%$ (keine Besteuerung) und $100 \%$ (jegliche Einnahmen müssen an den Staat abgeführt werden) einnehmen:

TAXREV $=\mathrm{f}(\mathrm{b})$

$0 \leq \mathrm{b} \leq 100 \%$

Bis zu einem kritischen Steuersatz $\left(b_{c}\right)$ hat die Kurve eine degressiv ansteigende Form:

Für $b<b_{c}$ gilt:

$\frac{\partial \mathrm{TAXREV}}{\partial \mathrm{b}}>0$

Genau beim kritischen Steuersatz $\left(b_{c}\right)$ wird der Grenzertrag der Steuereinnahmen jedoch Null:

Für $b=b_{c}$ gilt:

$\frac{\partial \mathrm{TAXREV}}{\partial \mathrm{b}}=0$

Jenseits dieses kritischen Steuersatzes $\left(b_{c}\right)$ wird die Steigung der Funktion negativ:

Für $b>b_{c}$ gilt:

$\frac{\partial \mathrm{TAXREV}}{\partial \mathrm{b}}<0$

Interessant sind die Annahmen, die hinter dem Kurvenverlauf der Laffer-Kurve stekken.

Tobin zeigt für die Herleitung der Laffer-Kurve die zugrundeliegenden Prämissen. ${ }^{60}$ Es liegt die Vermutung nahe, daß sich Tobin hierbei auf die Ausführungen von Laffer stützte.

.The model assumes that the supply of factors of production to the market sector is determined in part by the net-of-tax factor rewards; the factor supply functions are therefore assumed to be upwardsloping. The demand for factors of production depends on their marginal products; given competition and factor mobility, the factor marginal products will be equal to the gross-of-tax factor rewards. Thus the optimal factor mix used in the production process will depend on relative factor rewards. Only two factors of production are assumed, say labor and capital, and market-sector production is assumed to consist of a simple good that we call market output." 61

60 Vgl. Tobin, J., Policies for Prosperity: Essays in a Keynesian Mode, Yes, Virginia, There Are Laffer Curves, Brighton 1987, S. 120-125.

61 Vgl. Laffer, A. B., W. P., Government Exactions and Revenue Deficiencies, in: Fink, R. H. (Hrsg.), Supply-Side Economics: A Critical Appraisal, Maryland 1982, S. 186. 
Für die Herleitung der Laffer Kurve gelten nach Tobin folgende Annahmen:62

- Es liegt eine Cobb-Douglas Produktionsfunktion mit zwei Produktionsfaktoren Arbeit $(\mathrm{N})$ und Kapital $(\mathrm{K})$ vor, wobei der Kapitaleinsatz kurzfristig als konstant angenommen wird und von substituierbaren Produktionsfaktoren ausgegangen wird. Die Produktionsfaktoren erzeugen ein einziges Marktgut.

- Das Faktorangebot ist determiniert durch die Faktorentgelte nach Steuern.

- Die Nachfrage nach Produktionsfaktoren ist durch das jeweilige Faktorgrenzprodukt bestimmt.

- Die Faktorgrenzprodukte entsprechen den Bruttofaktorentgelten.

Tobin entwickelte aus den Annahmen eine mathematische Herleitung der LafferKurve, die nachfolgend, um einige formalen Zwischenschritte ergänzt, dargestellt wird. ${ }^{33}$

1. Cobb-Douglas Produktionsfunktion:

$$
Y=K^{\alpha} N^{1-\alpha}
$$

2. Das Grenzprodukt des Faktors Arbeit und das Bruttoentgelt des Faktors Arbeit pro Einheit Arbeit sind, den Annahmen entsprechend, gleich und damit:

$$
(1-\alpha) K^{\alpha} N^{-\alpha}, \text { weil } \frac{\partial Y}{\partial N}=(1-\alpha) K^{\alpha} N^{-\alpha}
$$

3. Die Höhe der insgesamt ausgezahlten Bruttoentgelte entspricht:

$$
(1-\alpha) Y \operatorname{oder}(1-\alpha) K^{\alpha} N^{1-\alpha}
$$

4. Die Einführung eines Einkommensteuersatzes (t) bringt Einnahmen in der Höhe von:

$$
t Y=t K^{\alpha} N^{1-\alpha}
$$

5. Der Faktor Arbeit wird angeboten in Abhängigkeit zu realer Entlohnung nach Steuern (aw) mit einer angenommenen Elastizität von $\frac{1}{\beta}$ und :

$62 \mathrm{Vgl}$. zu den Annahmen insbesondere auch: Wendisch, P., Supply-Side Policy, Theoretische Grundlagen und prozeßpolitische Konsistenz, Frankfurt am Main 1984, S. 101.

$63 \mathrm{Vgl}$. Tobin, J., Policies for Prosperity: Essays in a Keynesian Mode, Yes, Virginia, There Are Laffer Curves, Brighton 1987. S. 122-124. 
$N=(a w)^{1 / \beta}$, potenziert mit $\beta$ ergibt:

$\mathrm{N}^{\beta}=a w$, multipliziert mit $\mathrm{N}$ und aufgelöst nach $\mathrm{Nw}$ :

$N w=\frac{1}{a} N^{1+\beta}$

6. Für die Entlohnung nach Steuern verbleibt somit die Größe von:

$$
\begin{aligned}
& N w=\frac{1}{a} N^{1+\beta}, \text { das den besteuerten Bruttoentgelten nach Steuern entspricht } \\
& N w=\frac{1}{a} N^{1+\beta}=(1-t)(1-\alpha) K^{\alpha} N^{1-\alpha}
\end{aligned}
$$

7. Sei nun $u=(1-t)$, so ergibt sich durch umformen und logarithmieren:

$\frac{1}{a} N^{1+\beta}=u(1-\alpha) K^{\alpha} N^{1-\alpha}$, multipliziert mit a und $\frac{1}{\mathrm{~N}^{1-\alpha}}$ :

$\frac{N^{1+\beta}}{N^{1-\alpha}}=u(1-\alpha) a K^{\alpha}$, das läßt sich auch schreiben als

$N^{\alpha+\beta}=u(1-\alpha) a K^{\alpha}$, logarithmiert und multipliziert mit $\frac{1}{\alpha+\beta}$ ergibt:

$\log N=\frac{1}{\alpha+\beta} \log u+\frac{1}{\alpha+\beta} \log (1-\alpha) a K^{\alpha}$,

8. Die Steuereinnahmen (TR) ergeben sich aus, wobei $t=1-u$ :

$T R=(1-u) K^{\alpha} N^{1-\alpha}$, logarithmiert ergibt:

$\log \mathrm{TR}=\log (1-\mathrm{u})+(1-\alpha) \log \mathrm{N}+\log \mathrm{K}^{\alpha}$, setzt man nun das Ergebnis aus 7.

für $\log \mathrm{N}$ ein:

$\log T R=\log (1-u)+(1-\alpha)\left(\frac{1}{\alpha+\beta} \log u+\frac{1}{\alpha+\beta} \log (1-\alpha) a K^{\alpha}\right)+\log K^{\alpha}$ 
gem ä $B$ der Annahme sei der Kapitaleinsatz kurzfristig konstant,

dann gilt:

$\log \mathrm{TR}=\log (1-u)+\frac{1-\alpha}{\alpha+\beta} \log u+$ Konstante

9. Die Laffer-Kurve beschreibt den Zusammenhang zwischen Steuereinnahmen (TR) und Steuersatz (t), wobei von besonderem Interesse der Scheitelpunkt der Kurve ist. In diesem Punkt $\left(t^{*}\right)$ ist die Steigung der Kurve gleich Null. Der Grenzertrag der Steuereinnahmen ist in $t^{*}$ gleich Null.

Für jeden Steuersatz $t$, der größer als $t^{*}$ ist, gilt, daß die Steuereinnahmen zurückgehen; man befindet sich dann im prohibitiven Teil der Laffer-Kurve. Aus den bisherigen Herleitungen ermittelt sich dieser kritische Steuersatz $\left(t^{*}\right)$ aus dem Ergebnis für $\log \mathrm{TR}$, wobei u durch t ausgedrückt wird:

$\log T R=\log t+\frac{1-\alpha}{\alpha+\beta} \log (1-t)+$ Konstante, nach t differenziert:

$\frac{\partial \log \mathrm{TR}}{\partial \mathrm{t}}=\frac{1}{t}-\frac{1-\alpha}{\alpha+\beta} \frac{1}{1-t}$, auf den Hauptnenner gebracht:

$\frac{\partial \log T R}{\partial t}=\frac{\alpha+\beta-t(1+\beta)}{t(1-t)(\alpha+\beta)}$, nun wird $\mathrm{t}^{*}$ ermittelt, hierfür muß 1. Ableitung gleich

Null sein:

$\frac{\partial \log T R}{\partial t}=0$; das ist dann der Fall, wenn der Zä hler gleich Null wird:

$\alpha+\beta-t(\beta+1)=0$, aufgelöst nach $\mathrm{t}$ und damit erh ält man den Steuersatz $\mathrm{t}^{*}$ :

$\frac{(\alpha+\beta)}{(1+\beta)}=t *$

Die Steigung der Laffer-Kurve ist also solange positiv bis $t^{*}=\frac{(\alpha+\beta)}{(1+\beta)}$ ist.

Wird nun für $\alpha=\frac{1}{3}$ und $\beta=3$ gewählt, errechnet sich ein $t^{*}$ von 0,833 . 
Dieses Zahlenbeispiel wurde von Tobin gewählt. ${ }^{64}$ Nur im Bereich zwischen 0,833 und 1 wäre bei einer Senkung des Steuersatzes eine Erhöhung der Einnahmen möglich.

\subsubsection{Das Wedge-Modell}

Der "ökonomische Keil", der durch Steuererhebungen zwischen Angebot und Nachfrage auf dem Güter-, Arbeits- und Kapitalmarkt geschlagen wird, führt zu einer Veränderung der relativen Preise auf den Teilmärkten. Die Steuerpolitik muß einen Ausgleich zwischen der Einnahmenhöhe des Staates und der bestmöglichen Allokation auf den Märkten finden.

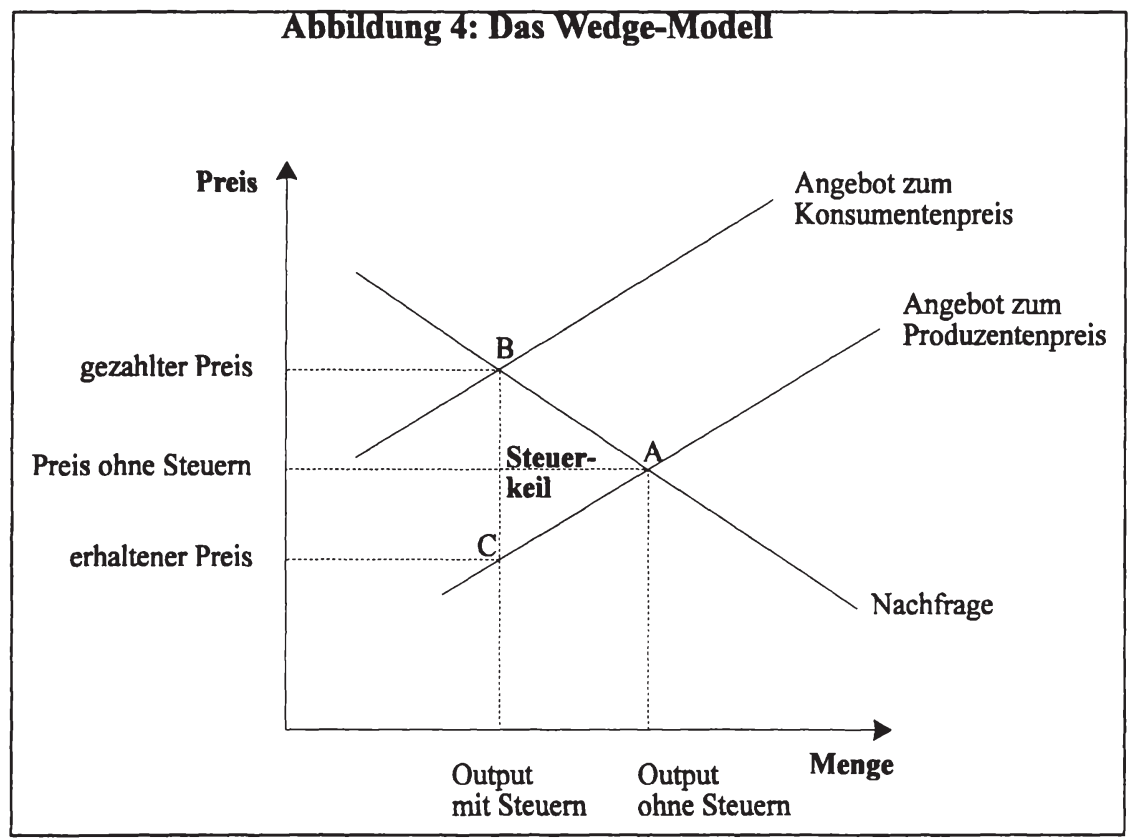

Quelle: eigene Darstellung, Wendisch, P., Supply-Side Policy, Theoretische Grundlagen und prozeßpolitische Konsistenz, Frankfurt am Main 1984, S. 64.

Indirekt wird damit auch der Geldpolitik ihre Rolle zugewiesen: sie hat sich neutral zu verhalten. Ist erst einmal die optimale relative Preisstruktur gefunden, wird automatisch über das Gleichgewicht auf den Märkten die Inflation eliminiert.

64 Ebenda, S. 124. 
Diese ökonomische Argumentationskette hat Laffer analytisch mit seinen "WedgeModellen" verdeutlicht. ${ }^{65}$

Das Keil (Wedge)-Modell läßt sich auf den Gütermarkt (Tax-Wedge), Arbeitsmarkt (Labor-Wedge) und Kapitalmarkt (Capital-Wedge) übertragen. Grundvoraussetzungen für die Wedge-Betrachtung sind die Annahmen, daß staatliche Aktivitäten die ökonomischen Anreize beeinflussen und da $B$ alle Wirtschaftssubjekte nach Nutzen- bzw. Gewinnmaximierung streben.

Insbesondere den Steuern kommt eine zentrale Funktion zu. Sie sind maßgeblich dafür verantwortlich, zu welchen Allokationseffekten es auf den Teilmärkten kommt. Das Angebots- bzw. Nachfrageverhalten hängt dabei von den Opportunitätskosten der jeweiligen Entscheidungsalternative ab. Steuern induzieren Kosten, was gleichbedeutend mit einer Veränderung der relativen Preise ist.

Die Differenz zwischen den Kosten, die eine Unternehmung für die Beschäftigung eines Arbeiters oder einer Einheit Kapital aufwenden muß, und dem, was ein Arbeiter oder Sparer netto erhält, ist der Steuerkeil. ${ }^{66}$ In der Abbildung wird der Steuerkeil von den Punkten A, B und C eingegrenzt. Eine Vergrößerung des Steuerkeils erhöht die Kosten für den Arbeitgeber, weil er höhere Löhne und/oder höhere Zinsbelastungen zu bezahlen hat. Folglich werden die Unternehmer weniger Arbeiter beschäftigen und weniger Kapital nachfragen. Auf der Angebotsseite bedeutet der Anstieg der Steuerlast eine Reduzierung der Netto-Löhne und eine Verringerung der Erträge der Sparer. In Folge wird weniger Arbeit angeboten werden und das Sparaufkommen wird zurückgehen.

Im Endergebnis verringert die Zunahme des Steuerkeils sowohl die Nachfrage nach Produktionsfaktoren als auch deren Angebot. Gesamtwirtschaftlich heißt dies, daß die Beschäftigung zurückgeht, weniger investiert und weniger produziert wird.

\subsubsection{Das Iso-Produktions-/Einnahmen-Modell}

Laffer hat im Rahmen dieses Ansatzes die Annahmen, die er für die Laffer-Kurve getroffen hatte (Kapitel 2.2.5.2), aufgegriffen und inhaltlich spezifiziert; die Auswirkungen einer Steuersatzänderung bei einzelnen Produktionsfaktoren auf das Produktionsniveau (Output) wurden dabei analysiert. ${ }^{67}$

65 Vgl. Laffer, A. B., Supply-Side Economics, in: Financial Analysts Joumal, September 1981, S. 33. und Wendisch, P., Supply-Side Policy, Theoretische Grundlagen und prozeßpolitische Konsistenz, Frankfurt am Main 1984, S. 64.

66 Die Steuern sind bei den Supply-Sidern der Hauptbestandteil des Wedge. Weiterhin zählen sie aber auch Subventionen, staatliche Regulierungen und die Höhe der Staatsausgaben hinzu, die nach ihrer Meinung marktbehindernd wirken.

67 Vgl. Laffer, A. B., Supply-Side Economics, in: Financial Analysts Journal, September 1981, S. 32. und Wendisch, P., Supply-Side Policy, Theoretische Grundlagen und prozeßpolitische Konsistenz, Frankfurt am Main 1984, S. 101-105. 
Hierfür wählte Laffer ein Modell mit zwei Produktionsfaktoren, Arbeit und Kapital, die ein Marktgut (ein Produktionsniveau) erzeugen; beide Produktionsfaktoren werden besteuert. Der Gesamtsteuersatz ergibt sich aus dem gewichteten Durchschnitt der beiden Einzelsteuersätze. Daraus leitete Laffer ab, daß es zahlreiche Kombinationen dieser beiden Einzelsteuersätze gibt, die zu dem gleichen Steuersatz führen und damit zu der gleichen Menge an Output.

„Thus it is apparent that there are numerous combinations of tax rates on each of the factors of production that yield the same product tax rate. That is, there are numerous combinations of factor tax rates that yield the same level of output." 68

Dieser Zusammenhang läßt sich an einem Iso-Output-Diagramm aufzeigen.

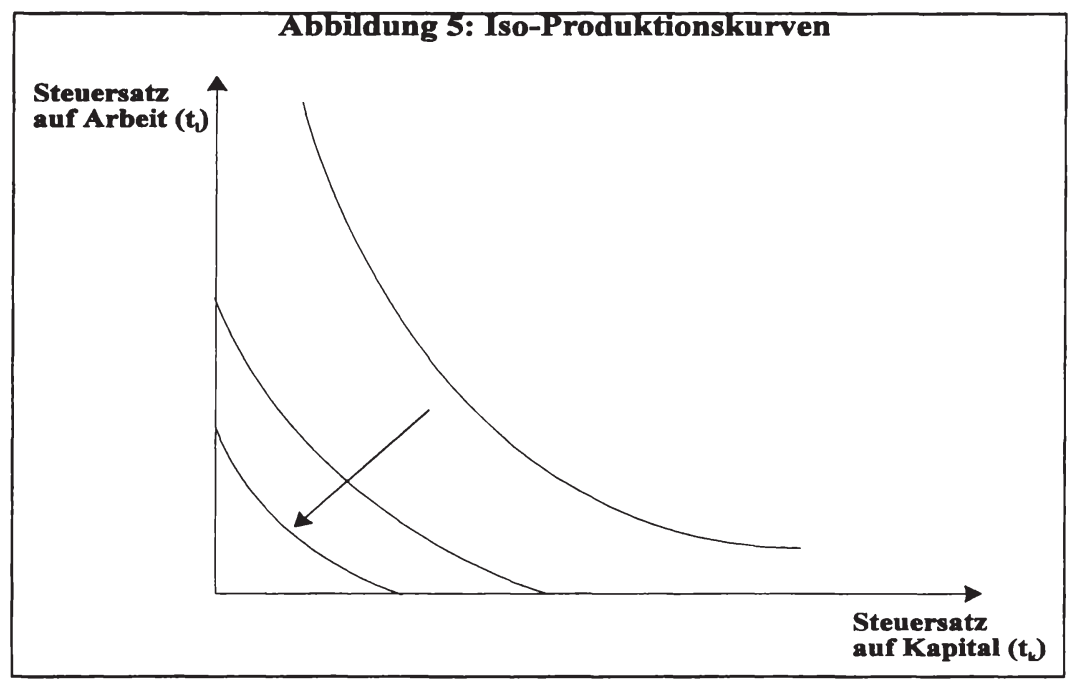

Quelle: Laffer, A. B., Government Exactions and Revenue Deficiencies, in: Fink, R. H. (Hrsg.), Supply-Side Economics: A Critical Appraisal, Maryland 1982, S. 187, eigene Darstellung.

Auf der Abszisse ist der Einzelsteuersatz auf den Produktionsfaktor Kapital $t_{k}$ abgetragen. Auf der Ordinate steht der Einzelsteuersatz auf den Faktor Arbeit t $t_{1}$. Die IsoOutput-Linien innerhalb des Diagramms stellen verschiedene Kombinationen der beiden Einzelsteuersätze dar, die denselben Gesamtsteuersatz ergeben. Der Pfeil innerhalb des Diagramms zeigt dabei in Richtung eines steigenden Outputs, da durch fallende Steuersätze ein höherer Output erzeugt wird. Die Iso-Output-Linien berühren die Ach-

68 Vgl. Laffer, A. B., W. P., Government Exactions and Revenue Deficiencies, in: Fink, R. H. (Hrsg.), Supply-Side Economics: A Critical Appraisal, Maryland 1982, S. 186. 
sen, weil auch dann produziert wird, wenn ein Produktionsfaktor nicht und der andere voll besteuert wird.

Die Konkavität der Isoquanten steht für die Annahme, daß die Grenzrate der Substitution zwischen den Faktorsteuersätzen abnimmt.

Im nächsten Schritt analysierte Laffer die Auswirkungen einer Erhöhung des Steuersatzes auf den Produktionsfaktor Arbeit, der die Höhe des Gesamtoutputs unverändert läßt. Dies führt zu zwei Effekten:

\section{Skaleneffekt:}

Durch die Verteuerung des Faktors Arbeit erhöht sich der Preis des Marktgutes und reduziert dadurch dessen Produktionsvolumen. Dies führt in Folge dazu, daß das Beschäftigungsvolumen von beiden Produktionsfaktoren zurückgeht.

\section{Substitutionseffekt:}

Die Verteuerung des Faktors Arbeit macht den Einsatz des Produktionsfaktors Kapital relativ attraktiver, so daß ein bestimmter Anteil des Faktors Arbeit durch den Faktor Kapital substituiert wird.

Die Überlagerung dieser beiden Effekte hat somit insgesamt eine starke Reduzierung des Faktors Arbeit zur Folge. Laffer ging von einer Dominanz des Skaleneffektes aus, dadurch reduziert sich zusätzlich auch der Produktionsfaktor Kapital.

Die Zunahme des Steuerkeils löst folgende Effekte aus:

- Pro Arbeitnehmer wird ein größeres Steuereinkommen erreicht.

Laffer bezeichnete dies als den arithmetischen Effekt der Besteuerung.

- Es werden insgesamt weniger Arbeitnehmer beschäftigt, was zu einem Rückgang der Steuereinnahmen führt.

Laffer bezeichnete dies als den direkten Feedback-Effekt der Besteuerung.

- Es wird als Folge des direkten Feedback-Effekts weniger Kapital eingesetzt und im weiteren Verlauf verstärkt sich der Rückgang der Einnahmen, da die Investitionen zurückgehen.

Laffer bezeichnete dies als den indirekten Feedback-Effekt der Besteuerung.

Falls der arithmetische Effekt überwiegt, führt dies zu steigenden Steuereinnahmen. Überwiegen die beiden anderen Effekte, führt dies aufgrund der sinkenden Steuerbasis, die aus dem Rückgang des Volumens der Produktionsfaktoren resultiert, zu sinkenden Steuereinnahmen.

Welche Auswirkung nun dominiert, ist nach Laffers Einschätzung ungewiß und hängt in der Praxis von weiteren Faktoren ab. Die Dauer der Steuerbelastung, die absolute Höhe der Steuerlast und der zeitliche Verlauf der Substitution der Produktionsfaktoren beeinflussen ebenso diesen Prozeß.

Um die Auswirkung einer Steuersatzerhöhung genauer zu analysieren, gebrauchte Laffer eine weitere Veranschaulichung, die in der Abbildung 6 dargestellte Iso-Ein- 
nahmen-Kurve. Die Iso-Einnahmen-Kurve zeigt die Auswirkungen einer Erhöhung der jeweiligen Einzelsteuersätze bei konstanten Steuereinnahmen.

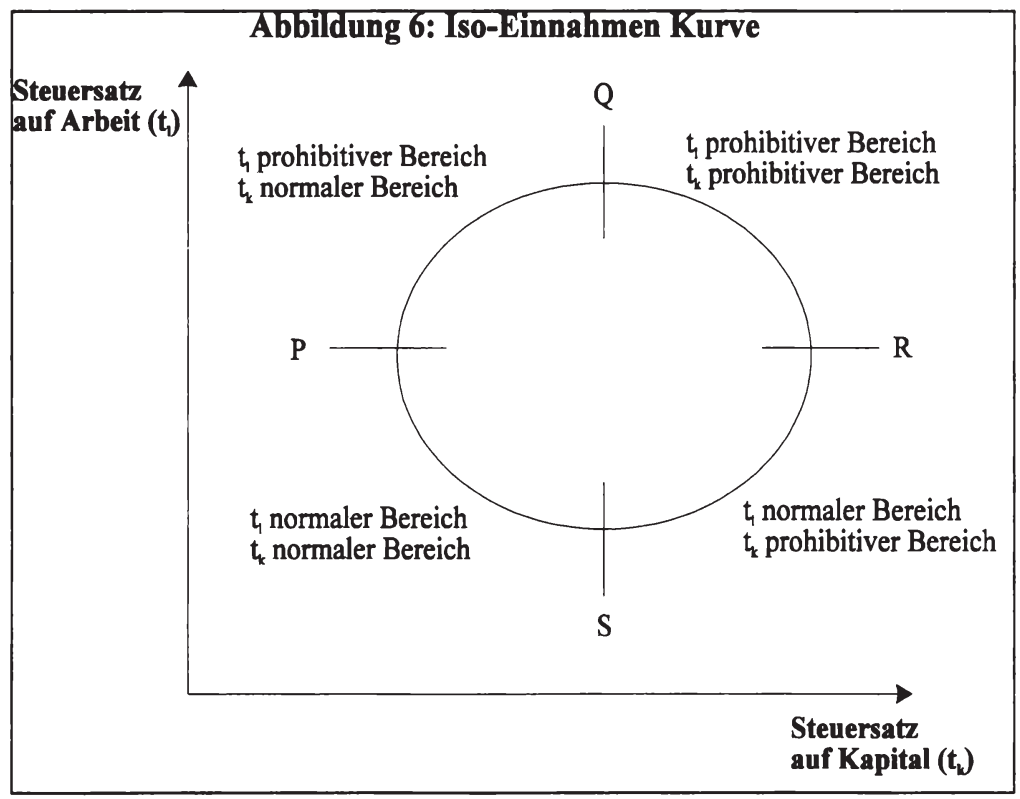

Quelle: Laffer, A. B.; Government Exactions and Revenue Deficiencies, in: Fink, R. H. (Hrsg.), Supply-Side Economics: A Critical Appraisal, Maryland 1982, S. 189, eigene Darstellung.

Auf der Abszisse ist hier wieder der Steuersatz auf Kapital $t_{k}$ und auf der Ordinate der Steuersatz auf Arbeit $t_{1}$ abgetragen. Die Iso-Revenue-Kurve stellt verschiedene Steuersatzkombinationen dar, aus denen das gleiche Steueraufkommen resultiert. Laffer unterschied vier Bereiche auf der Kurve:

1. PQ-Bereich: In dieser Region befindet sich der Steuersatz auf Arbeit im prohibitiven Bereich, während $t_{k}$ sich im normalen Bereich der Besteuerung bewegt. Eine Erhöhung des Steuersatzes auf Arbeit führt zu geringeren Steuereinnahmen und im Gegensatz dazu erhöht ein höherer Steuersatz auf Kapital die Steuereinnahmen. Die Steuereinnahmen bleiben dann konstant, wenn eine Steuersatzsenkung auf den Produktionsfaktor Arbeit begleitet wird von einer gleichzeitigen Senkung des Steuersatzes auf den Produktionsfaktor Kapital.

2. QR-Bereich: Im Unterschied zum PQ-Bereich befinden sich hier beide Steuersätze $t_{l}$ und $t_{k}$ im prohibitiven Bereich. Senkt man nun $t_{l}$, so führt dies zu steigenden Steuereinnahmen, so daß man $t_{k}$ erhöhen müßte, um wieder dasselbe Steueraufkommen zu erlangen. 
3. RS-Bereich: Der Steuersatz auf Kapital $t_{k}$ bewegt sich nun im ,prohibitive range“ und $t_{l}$ befindet sich im "normal range“. Hier führt eine Senkung von $t_{l}$ zu niedrigeren Steuereinnahmen, die durch eine gleichzeitige Senkung von $t_{k}$ wieder kompensiert werden können.

4. PS-Bereich: Beide Steuersätze befinden sich im normalen Bereich der LafferKurve. Die Senkung eines Steuersatzes führt zu niedrigeren Einnahmen, die nur durch eine Erhöhung des anderen Steuersatzes ausgeglichen werden können.

In allen Bereichen, außer im PS-Bereich, können beide Einzelsteuersätze gesenkt werden, ohne eine Verringerung der Steuereinnahmen herbeizuführen.

„From the realationship postulated in this tax ellipse, we see that in any region other than PS a lowering of at least one tax rate can be accompanied by a lowering of the other tax rate without reducing total revenue or spending. Only in the PS range does a lowering of one tax rate necessitate a raising of the other rate in order to maintain the same total revenue. "69

Für den PS-Bereich kam Laffer zu weiteren Schlußfolgerungen. Eine Senkung von $t_{1}$ bei einer gleichzeitiger Erhöhung von $t_{k}$ hat zur Folge, da $B$ weniger Kapital eingesetzt wird. In Folge sinkt die Nachfrage nach Arbeit und die gesamtwirtschaftliche Höhe der Bruttolöhne der Arbeitnehmer verringert sich. Damit ergibt sich trotz der Steuersatzsenkung auf den Faktor Arbeit eine negative Wirkung auf die Beschäftigungshöhe.

Im PS-Bereich wird deutlich, daß Laffer zu einer zweideutigen Interpretation des Abhängigkeitsverhältnisses bezüglich der beiden Produktionsfaktoren Arbeit und Kapital gelangt. Der Faktor Kapital kann nicht durch den Faktor Arbeit ersetzt werden, ausgehend von einer Erhöhung des Steuersatzes auf Kapital, was auf ein limitationales Abhängigkeitsverhältnis zwischen den Produktionsfaktoren hinweist. Wenn man jedoch den Steuersatz auf Arbeit erhöht, kann von einem substitutionalen Verhältnis ausgegangen werden. Das bedeutet, daß bei einer Erhöhung des Steuersatzes auf Arbeit dieser Faktor problemlos durch den Faktor Kapital ersetzt werden kann, jedoch nicht umgekehrt. Eine Politik, die darauf abzielt, den Faktor Arbeit steuerlich zu entlasten und die Höhe der Steuereinnahmen trotzdem über eine höhere Besteuerung von Kapital konstant zu halten, führt zu höherer Arbeitslosigkeit.

.. Similarly, taxing the rich is sometimes a good way to further impoverish the poor. "70

Laffer hat die Iso-Produktions-Graphik und die Iso-Einnahmen-Kurve in einem Schaubild zusammengefaßt. (Abbildung 7)

Abbildung 7 zeigt den Steuersatz auf Kapital $t_{k}$ auf der Abszisse und den Steuersatz auf Arbeit $t_{l}$ auf der Ordinate, wobei die Iso-Produktion-Linien linear dargestellt sind. Es gibt für jedes Steueraufkommensniveau nur genau eine Steuersatzkombination, die auch gleichzeitig den Output maximiert. Ein solcher, durch diese Steuersatzkombination festgelegter Punkt ist O*, der den Berührungspunkt zwischen Iso-Einnahmen-Linie 1

69 Ebenda, S. 189.

70 Ebenda, S. 191. 
und Iso-Produktion-Linie 1 darstellt. Durch die verschiedenen Berührungspunkte ergibt sich eine Linie optimaler Steuersatzkombinationen, die vom Ursprung bis zu dem hier mit E gekennzeichneten Endpunkt führt. In diesem Endpunkt, der das überhaupt maximale erreichbare Steuereinkommen repräsentiert, kann keine wie auch immer geartete Veränderung der Einzelsteuersätze vorgenommen werden, ohne daß sich automatisch das maximale Steueraufkommen durch diese Veränderung verringert. Insgesamt kann bei allen Steuersatzkombinationen, die nicht auf dieser Linie optimaler Steuersatzkombinationen liegen, durch Veränderung dieser Kombination entweder ein größeres Steueraufkommen - unter Konstanthaltung des Outputs - oder eine höheres Outputniveau unter Konstanthaltung der Steuereinnahmen - erreicht werden.

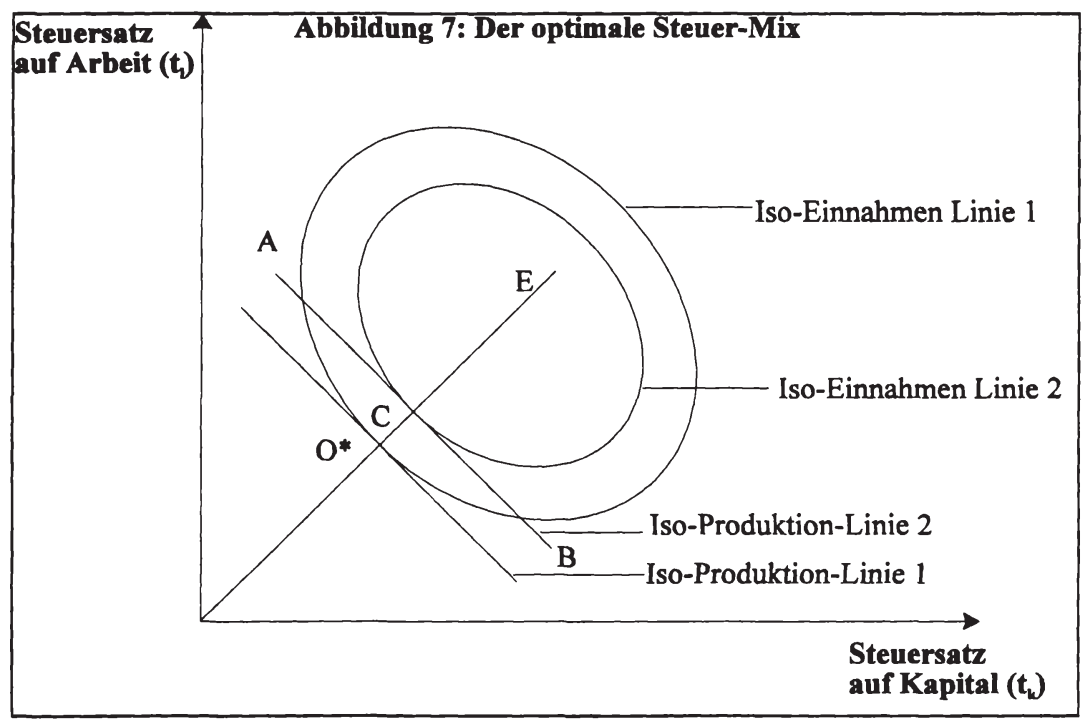

Quelle: Laffer, A. B., Government Exactions and Revenue Deficiencies, in: Fink, R. H. (Hrsg.), Supply-Side Economics: A Critical Appraisal, Maryland 1982, S. 190, eigene Darstellung.

Das Laffer-Modell enthält implizit auch eine Wachstumsmöglichkeit für die Volkswirtschaft. Eine geschickte Steuerpolitik, die die Faktorsteuersätze so festsetzt, daß, z.B. ausgehend von der Abbildung 7, die Wirtschaft sich von den Punkten A oder B zum Punkt $\mathrm{O}^{*}$ bewegt, kann bei gleicher Einnahmenhöhe einen höheren Output erzielen.

Das erweiterte Laffer-Modell lieferte mit der vorgestellten Argumentationslinie einen weiteren Beweis im Sinne der Supply-Sider dafür, daß Steuersenkungen immer sinnvoll sind; insbesondere dann wenn sich die Volkswirtschaft im prohibitiven Bereich der Besteuerung befindet. Steuersenkungen führen zudem immer zu einem Wachstum 
der Volkswirtschaft, selbst im normalen Bereich der Besteuerung. Die positiven Effekte, die auf der Angebotsseite durch eine geringere Besteuerung ausgelöst werden, übertreffen die negativen Effekte, die möglicherweise durch einen geringeren Ausgabenspielraum der öffentlichen Haushalte entstehen.

\subsubsection{Auswirkungen der angebotsorientierten Steuerpolitik auf die Angebotskurve}

Die zentrale Idee der angebotsorientierten Wirtschaftspolitik ist, das zeigten die LafferModelle, daß verschiedene Arten von Steuersenkungen letztlich einen Anstieg des aggregierten gesamtwirtschaftlichen Angebots bewirken. ${ }^{71}$ Voraussetzung für diese Annahme ist, daß die Wirtschaftssubjekte auf diesen steuerlichen Anreiz in der Form reagieren, daß sie das Geamtangebot an Arbeit und Kapital erhöhen.

Abbildung 8 illustriert diese Idee an einer aggregierten Angebots (AS-Kurve)- und Nachfragekurve (AD-Kurve).

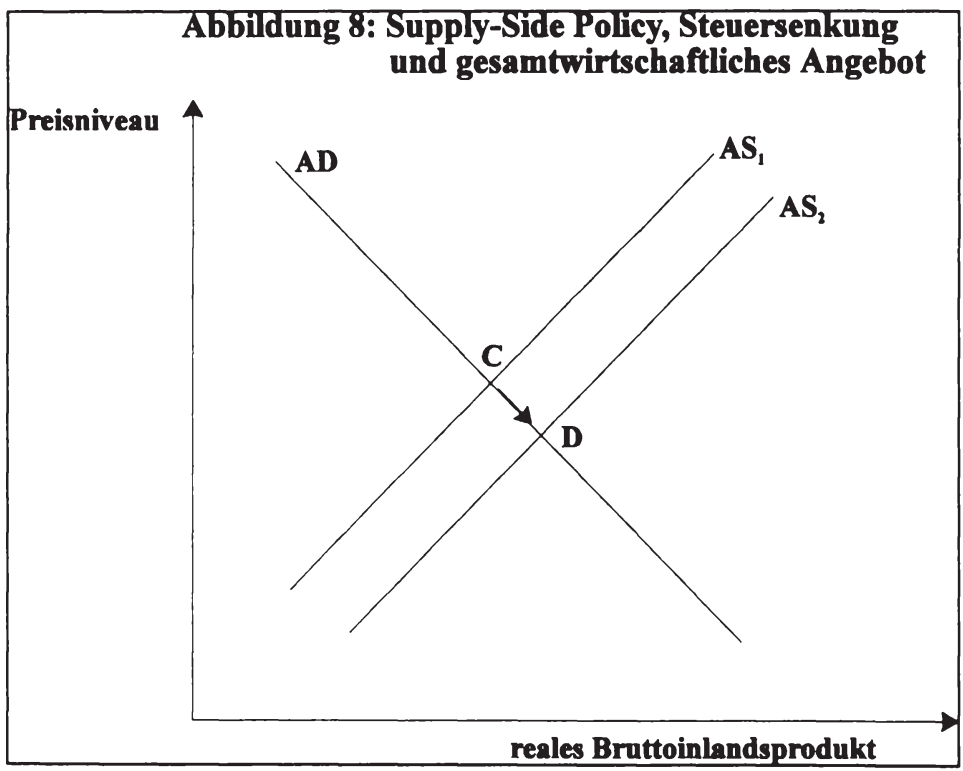

Quelle: Baumol, W. J., Blinder, A. S., Economics, Principles and Policy, sixth edition, 1994, S. 703. eigene Darstellung.

71 Vgl. Baumol, W. J., Blinder, A. S., Economics, Principles and Policy, sixth edition, 1994, S. $702-$ 704 . 
In der Ausgangssituation ohne eine angebotsorientierte Steuersenkung befindet sich die Volkswirtschaft im Punkt C (Schnittpunkt der AD-Kurve mit der $\mathrm{AS}_{1}$-Kurve). Geht man nun davon aus, daß es zu Steuersenkungen kommt und die Wirtschaft so reagiert, wie es sich die Supply-Sider vorstellen, wird sich die Angebotskurve in Pfeilrichtung $\left(\mathrm{AS}_{2}\right.$-Kurve) verschieben. Ein neuer Gleichgewichtspunkt zwischen gesamtwirtschaftlicher Nachfrage und gesamtwirtschaftlichem Angebot wird dann beispielsweise im Punkt D ereicht werden. Im Punkt D weist die Volkswirtschaft ein höheres Volkseinkommen bei einem geringeren Preisniveau als in der Ausgangssituation (Punkt C) aus. Das ökonomische Ziel der Steuersenkung wäre erreicht, zumindest graphisch.

Die Frage bleibt jedoch unbeantwortet, ob die Wirtschaft genau so auf Steuersenkungen reagiert, wie sich das die Supply-Sider vorstellen, damit dieses theoretische Szenario auch tatsächlich so eintritt.

\subsection{Kritik an der Theorie der Supply-Side Policy}

Martin Feldstein, der von 1982-84 Vorsitzender des Council of Economic Advisers war, kommt in seiner Nachbetrachtung der amerikanischen Wirtschaftspolitik der 80er Jahre zu dem Ergebnis, daß die extremen Angebotstheoretiker, die an die Laffer-Kurve glaubten, der Supply-Side Theorie geschadet hätten.

„The rhetoric of self-financing tax cuts nevertheless continued during the 1980 presidential campaign and was later part of the administration's effort to sell the tax package to Congress and the nation. The implausibility of the claim that the tax cut would be self-financing clearly did not hamper the ability of the new Reagan administration to enact its package, but it did complicate my subsequent job as CEA chairman in defending the tax package as good economics despite its obvious failure to raise revenue. And just when an increasing number of mainstream economists were accepting the traditional ..supply-side" view that incentives are important and that high tax rates do not raise correspondingly high revenues, the supply-side extremists gave supply-side arguments in general a bad name."72

Einige Supply-Sider bestreiten mittlerweile, daß sie den Zusammenhang zwischen Steuersenkungen und höheren Steuereinnahmen propagiert hätten. Robert Bartley relativiert in seinem Buch „The Seven Fat Years“ die Bedeutung der Laffer-Kurve. Er kommt dabei zu dem Schluß, daß jedes politische Ziel ein Hilfsmittel benötigt, an dem die Wirkung verdeutlicht werden kann.

.For every policy goal, you need a policy lever“"73

Mit dem Hebel der Laffer-Kurve gelang es den Angebotstheoretikern, ihre wirtschaftspolitischen Vorstellungen öffentlichkeitswirksam publik zu machen. Im nachhinein erklären nun die Supply-Sider, daß auch ihnen durchaus klar war, daß bei einer

72 Vgl. Feldstein, M., American Economic Policy in the 1980s: A personal view, in: Feldstein, M. (Hrsg.), American Economic Policy in the 1980s, Chicago 1994, S. 25.

73 Vgl. Bartley, R. L., The seven fat years-and how to do it again, New York 1995, S. 59. 
Wirtschaft, welche sich im normalen Bereich der Laffer-Kurve befindet, Steuersenkungen ein Defizit erzeugen. Einige gehen sogar soweit und behaupten, daß die SupplySider nie gesagt hätten, daß sich Steuersenkungen gegebenenfalls selbstfinanzieren. ${ }^{74}$

Unzweifelhaft entspricht jedoch diese Einschätzung nicht der Wahrheit. Laffer hat 1981 erklärt:

.It is reasonable to conclude that each of the proposed 10 percent reductions in tax rates would , in terms of overall tax revenues, be selffinancing in less than two years. “75

Selbst wenn man die späteren Rechtfertigungen der Supply-Sider als glaubwürdig erachten würde, bleibt eine Frage unbeantwortet. Warum haben die Protagonisten der Angebotstheorie keinen Versuch unternommen, den Verlauf der Laffer-Kurve für die U.S.A. zu ermitteln? Stattdessen wurde mit historischen Referenzbeispielen die Notwendigkeit von Steuersenkungen begründet. ${ }^{76}$ Es wurde jedoch implizit davon ausgegangen, daß sich die amerikanische Volkswirtschaft im prohibitiven Bereich der Besteuerung zu Beginn der 80er Jahre befand.

..We are well within this range at present. ${ }^{77}$

Auf dieser Grundlage bedeuteten die umfassenden Steuersenkungen, die während der Reagan Präsidentschaft vorgenommen wurden, ein nicht zu kalkulierendes Risiko, da die Supply-Sider einen Beweis schuldig blieben, der belegte, daß sich die amerikanische Volkswirtschaft tatsächlich im prohibitiven Bereich der Besteuerung befand.

Neben diesen grundsätzlichen Einwänden über die Wirksamkeit einer angebotsorientierten Wirtschaftspolitik lassen sich weitere Kritikpunkte finden. Ausgangspunkt für eine kritische Analyse bilden auch hierbei die Kernaussagen der Supply-Side Theorie und die daraus abgeleiteten Argumentationsketten:

- Innerhalb der Angebotstheorie und ihrer Wirkungsmechanismen spielen die Steuererhebung bzw. die vom Staat auferlegten Steuern die zentrale und entscheidende Rolle.

- Ausgehend von der Annahme, daß sich die Wirtschaftssubjekte in ihren individuellen Wahlakten und Entscheidungen über ihr Arbeitsangebot, ihre Konsum- und Sparquote und ihre Investitionsneigungen von gewissen Anreizen und Bedingungen beeinflussen lassen, stellt sich die Frage, durch welche Maßnahmen man diese Anreizstruktur so verändern kann, daß sich der Einzelne in eine gewünschte Richtung entscheidet.

$74 \mathrm{Vgl}$. Feldstein. M.. American Economic Policy in the 1980s: A personal view, in: Feldstein. M. (Hrsg.). American Economic Policy in the 1980s, Chicago 1994, S. 25.

75 Vgl. Laffer, A. B., W. P., Government Exactions and Revenue Deficiencies, in: Fink. R. H. (Hrsg.). Supply-Side Economics: A Critical Appraisal, Maryland 1982. S. 201.

76 Ebenda. S. 194-201.

$77 \mathrm{Vgl}$. Laffer. A. B., Statement prepared for the Joint Economic Committee, in: The economics of the tax revolt: A reader, Laffer, A. B. und Seymour, J. P. (Hrsg.), New York 1977, S. 79. 
- Die Supply-Sider sehen in der Besteuerung den entscheidenden Faktor zur Beeinflussung der Anreizstruktur und damit zur Beeinflussung der einzelnen Wirtschaftsteilnehmer.

- Steuersenkungen verändern die relative Preisstruktur zugunsten eines vermehrten Arbeitsangebotes und einer größeren Spar- und Investitionsneigung. Weiter wird unterstellt, daß diese steuerinduzierte Veränderung der Produktionsfaktoren Arbeit und Kapital zu einer Ausweitung des Produktionspotentials führen.

- Entscheidend für die Wirksamkeit dieser Argumentationslinie sind die Elastizitäten mit der die Arbeits-, Spar- und Investitionstätigkeiten auf die Steuersatzänderungen reagieren.

\subsubsection{Steuerwirkungen auf dem Arbeitsmarkt}

Die Angebotstheorie unterstellt, daß eine Verringerung der Steuerbelastung aufgrund der veränderten relativen Preise eine Substitution von Freizeit durch Arbeit bewirkt und damit eine positive Auswirkung auf das Arbeitsangebot ausgelöst wird. Weiter wird von den Supply-Sidern angenommen, da $\beta$ der Substitutionseffekt den Einkommenseffekt dominiert.

Jedoch könnte gleichermaßen eine Verringerung des Arbeitsangebotes als Ergebnis einer Steuersatzsenkung eintreten. Dies wäre dann der Fall, wenn der Einkommenseffekt stärker wirkt als der Substitutionseffekt. Dann würden die Anreizeffekte einer Verringerung der Steuersätze in die entgegengesetzte Richtung wirken, denn die Wirtschaftssubjekte könnten ihre angestrebten Einkommensziele schneller und mit weniger Arbeitseinsatz erreichen. Der Einzelne würde sich bei steigendem Nettoeinkommen für mehr Freizeit entscheiden. ${ }^{78}$

Laffer bestreitet das Auftreten des Einkommenseffektes nicht, aber er geht davon aus, daß dieser Einkommenseffekt durch die gleichzeitige Kürzung der Transferausgaben (negativer Einkommenseffekt) vollständig ausgeglichen würde. Letztendlich kompensiert sich, so Laffer, der Einkommenseffekt über alle Wirtschaftssubjekte hinweg zu Null, während der Substitutionseffekt über den gesamten Bereich der möglichen Einkommen verhaltensbestimmend sei. ${ }^{79}$

Die vollständige Kompensation des Einkommenseffektes muß jedoch stark bezweifelt werden. Zum einen kann der Transferbereich der öffentlichen Hand nicht kurzfristig umgestaltet werden; zum anderen ist die von Laffer unterstellte weitgehende Identität von steuerlich Entlasteten und von der Ausgabenkürzung Belasteten unrealistisch. Aufgrund der Progressivität des Einkommensteuersystems führen Steuerentlastungen

78 Vgl. Roberts, P.C., Supply-Side Economics: An Assesment of The American Experience In The 1980s, Febuar 1989, National Westminster Bank Quarterly Review, S. 66.

79 Vgl. Laffer, A. B., Supply-Side Economics, in: Financial Analysts Journal 37. 1981, October, S. 41 und Laffer, A. B., An Equilibrium Rational Macroeconomics Framework, in: Kamrany, N. M., Day, R. H. (Hrsg.), Economic Issues of the Eighties, Baltimore 1979, S. 52. 
für hohe Einkommen zu relativ stärkeren Entlastungen als entgegengerichtete Transferzahlungen des Staates für untere Einkommensgruppen. ${ }^{80}$

„With the progressivity of income taxes, redistributions from those in the upper levels must lead to a greater positive income effect than the negative impacts felt by the receivers of these funds, who are clustered in much lower, if any, marginal tax brackets. " 81

Die Frage nach der Dominanz einer der beiden Effekte ist empirisch sehr umstritten. 82

Einige Studien untersuchten die Reaktion der Beschäftigten auf Steueränderungen und ermittelten Elastizitäten für das Arbeitsangebot.

Die Ergebniss der Untersuchungen lassen sich in soweit zusammenfassen, als daß eine Veränderung des marginalen Steuersatzes, wenn überhaupt, nur eine sehr geringe Auswirkung auf das Arbeitsangebotsverhalten der Wirtschaftssubjekte ausübt. ${ }^{83}$

Fullerton gibt als Resultat seiner Analyse für die aggregierte Arbeitsangebotsfunktion eine Arbeitsangebotselastizität von $0,15 \%$ an. ${ }^{84}$ Das bedeutet, daß für eine Steuersenkung, bei der sich der Reallohn um $10 \%$ erhöht, eine Steigerung des Arbeitsangebotes um $1,5 \%$ erwartet werden kann.

Das Arbeitsangebotsverhalten der einzelnen Wirtschaftssubjekte wird nach den Ergebnissen der empirischen Studien nicht in erster Linie von der Steuerpolitik determiniert, sondern von einer Vielzahl nicht quantifizierbarer Einflüsse.

So untersuchte Koch die Reaktion von Selbständigen auf eine Einkommensteuersenkung und kam zu dem Ergebnis, daß bei dieser Berufsgruppe nicht die Höhe des Steuersatzes, sondern in erster Linie die Beanspruchung durch deren Kunden, Mandanten oder Patienten das Ausmaß ihrer Wochenarbeitszeit bestimmt. ${ }^{85}$ Zudem, so Koch, gilt eine Ausweitung des Arbeitsangebotes bei einer durchschnittlichen Wochenarbeitszeit der Selbständigen von über sechzig Stunden als sehr unwahrscheinlich.

Aber auch unter den abhängig Beschäftigten tritt nach den empirischen Untersuchungen nur eine sehr schwache Reaktion auf eine Lohnsteuersenkung auf. Nur ungefähr acht Prozent der Beschäftigten würden ihre Arbeitszeit ausdehnen wollen, jedoch genausoviel würden ihr Arbeitsangebot einschränken. ${ }^{86}$

80 Vgl. Moszer,M.: A Comment on the Laffer Model, in: Fink, R. H. (Hrsg.), Supply-Side Economics: A Critical Appraisal; Maryland 1982, S. 220.

81 Ebenda.

82 Vgl. Barth, J. R.: The Reagan Program for economic recovery: Economic Rationale; Federal Reserve Bank of Atlanta, Economic Review, September 1981, S. 8.

$83 \mathrm{Vgl}$. Brown, C.: Taxation and the incentive to work, Oxford 1983, S. 47, oder Fullerton. D.: On the possibility of an inverse relationship between tax rates and government revenues, in: Journal of Public Economics 19, 1982, S. 3-22.

$84 \mathrm{Vgl}$. Fullerton, D.: On the possibility of an inverse relationship between tax rates and government revenues, in: Journal of Public Economics 19, 1982, S. 19.

$85 \mathrm{Vgl}$. Koch, W. A. S.: Erhöhen Steuersenkungen die Leistungsbereitschaft?, in: Wirtschaftsdienst 65,1985 , S. 531-536.

86 Ebenda. 
Es muß berücksichtigt werden, daß die unterstellte Wahlmöglichkeit bezüglich des Ausmaßes des Arbeitseinsatzes nur theoretisch gegeben ist. Aufgrund tariflicher Regelungen und fester Arbeitszeiten, in denen selbst die Möglichkeit von Überstunden stark eingeschränkt ist, kann die Mehrzahl der Beschäftigten praktisch nicht über ihr Arbeitsangebot frei entscheiden. Die einzige Wahlmöglichkeit besteht demnach nur noch in der Aufnahme einer Beschäftigung oder dem Verharren in der Arbeitslosigkeit.

Die empirischen Untersuchungen konnten jedenfalls die Annahme einer hohen Elastizität und Reagibilität des Arbeitsangebotes auf eine Steueränderung, auch wenn sie theoretisch elegant erscheinen mag, nicht bestätigen.

\subsubsection{Steuersenkung und Kapitalangebot}

Für die Supply-Sider gilt der Substitutionsprozeß, der bei einer Veränderung des Steuersatzes einsetzt, auch als entscheidende Determinante für die Höhe des Kapitalangebots. Demnach erhöht eine Einkommensteuersenkung den Kapitalertrag und mindert den Gegenwartswert des Einkommens zugunsten seines Zukunftswertes. Die Wirtschaftssubjekte werden nun Gegenwartskonsum durch zukünftigen Konsum substituieren, was zu einer Erhöhung des Kapitalangebots führt. Aber auch hier kann rein theoretisch der Einkommenseffekt dominieren. Durch die steuerinduzierte Erhöhung der Kapitalerträge würde nun ein geringerer Sparbetrag ausreichen, um denselben $\mathrm{Zu}$ kunftskonsum zu erreichen wie zuvor. ${ }^{87}$ Der Einkommenseffekt bewirkt also eine $\mathrm{Zu}$ nahme des Gegenwartskonsums; die keynesianische Variante einer Steuersenkung, die sicherlich nicht im Sinne der Supply-Sider wäre.

„The ability of tax rate cuts from present levels to increase tax receipts, however, remains doubtful. Increases in output that follow tax cuts are principally demand- and multiplier related. Until substantial productivity changes and capacity increments from tax cuts be demonstrated, supply-side stimulation will play a secondary role in macro policy." 88

Eine steuerinduzierte Erhöhung des Kapitalertrags ist vergleichbar mit einer Zinserhöhung. So kann man die Frage nach der Dominanz einer der beiden Effekte auch über die empirische Untersuchung der Zinselastizität beantworten.

Es gibt eine Vielzahl von empirischen Analysen über die Zinselastizität; die Ergebnisse hierzu sind allerdings widersprüchlich.

87 Vgl. Barth, J. R.: The Reagan Program For Economic Recovery: Economic Rationale; Federal Reserve Bank of Atlanta, Economic Review, Sept. 1981, S. 6.

88 Vgl. Moszer,M.: A Comment on the Laffer Model, in: Fink, R. H. (Hrsg.), Supply-Side Economics: A Critical Appraisal; Maryland 1982, S. 222. 
Gegner der Angebotstheorie zitieren Untersuchungen wie die Analyse von Denison, die dem Sparverhalten nur eine sehr geringe Abhängigkeit von der Zinshöhe bescheinigt. ${ }^{89}$

Denison untersuchte das Sparverhalten der Haushalte und der Unternehmen und fand heraus, daß seit 1920 das Verhältnis des Sparvolumens zum Bruttosozialprodukt in den USA nahezu konstant geblieben ist. Die Angebotsökonomen hingegen beziehen sich auf eine Studie von Boskin, der eine Zinselastizität von ungefähr $0,4 \%$ angibt. ${ }^{90}$

Andere Analysen untersuchten das Sparverhalten getrennt nach den drei Sektoren Staat, Unternehmen und Private. Von Fürstenberg bestätigte in seiner Studie einerseits die Konstanz der privaten Sparquote, andererseits fand er zwischen den einzelnen Sektoren eine inverse Beziehung bezüglich des Sparverhaltens heraus. Trotz der Inelastizität der Gesamtsparquote ergaben sich signifikante Elastizitäten der einzelnen Komponenten.

Das Sparverhalten wird laut dieser Untersuchung von sehr vielen Faktoren bestimmt. Die Steuerbelastung ist dabei nur eine von vielen Determinanten. ${ }^{91}$

Das Ziel der Supply-Side Policy, die Sparquote bzw. das Kapitalangebot allein durch eine Senkung der Steuersätze deutlich zu erhöhen, ist nach den meisten empirischen Untersuchungen unrealistisch.

\subsubsection{Einflußfaktoren auf das Investitionsverhalten}

Nach Auffassung vieler Ökonomen ist der negative Einfluß einer Gewinnbesteuerung für das Investitionsverhalten in der Angebotstheorie überbewertet.

Neben dem Streben nach möglichst hohen Gewinnen besteht eine Vielzahl von Motiven, welche die Investitionsentscheidungen beeinflussen. Dazu gehören neben den ökonomischen Zielen auch soziale und ökologische Motive, wie zum Beispiel die Sicherung der Arbeitsplätze und betriebliches Engagement im Bereich des Umweltschutzes.

So zeigte Wittmann in einer Untersuchung, in der er mehrere Unternehmen nach ihren Investitionsmotiven befragte, daB dem steuerlichen Gesichtspunkt nur eine untergeordnete Rolle zukommt. ${ }^{92}$ Bei Erweiterungs- oder Ersatzinvestitionen sind die primären Bestimmungsgründe vor allem die Verbesserung der Kostensituation und der Pro-

$89 \mathrm{Vgl}$. Jetha, N.: Taxation and economic behaviour, Bulletin for International Fiscal Documentation, 34, 1980, S. 157.

90 Vgl. Boskin, M.: Taxation, Saving and the Rate of Interest, in: Journal of Political Economics, 86, 1978, S. 3-4.

91 Vgl. von Fürstenberg, G., Saving, in: Aaron, A., Pechman, J (Hrsg.), How taxes affect economic behaviour, Washington 1981, S. 327-402.

$92 \mathrm{Vgl}$. Wittmann, F., Die Berücksichtigung von Steuem bei Investitionsentscheidungen der Unternehmen, WSI-Mitteilungen, 39, 1986, S. 782 und Lamping, H.: Supply-Side Economics, Oldenburg, 1988, S. 72. 
duktqualität, aber auch die Einsparung von Arbeitsplätzen und die geschätzten Absatzerwartungen.

Die Kritik an der Angebotstheorie konzentriert sich also vor allem auf den Anspruch, mit dem Instrument der Besteuerung wirtschaftspolitische Ziele erreichen zu können. Durch die Veränderung von Steuersätzen können sicherlich Anreize geschaffen bzw. vermindert werden, aber die Steuererhebung ist nur einer von vielen Faktoren und Motiven, die auf die individuellen Entscheidungen der Wirtschaftssubjekte einwirken. Wie die empirischen Studien zeigen, ist der Einfluß der Besteuerung sowohl auf dem Arbeitsmarkt wie auch auf dem Kapitalmarkt sehr gering.

Dadurch wird die Wirksamkeit und die Glaubwürdigkeit der angebotstheoretischen Anreizmechanismen und der Angebotspolitik insgesamt in Frage gestellt.

\subsubsection{Kritische Bemerkungen zur Laffer-Kurve}

Die Laffer-Kurve beschreibt den Zusammenhang zwischen dem Steuersatz und den Steuereinnahmen des Staates.

Hinter dieser vereinfachten Darstellung verbirgt sich der gesamte angebotstheoretische Anreizmechanismus. Hierbei sind die Faktorelastizitäten bezüglich der Steuersatzänderungen von entscheidender Bedeutung. Die angebotspolitische Argumentation basiert auf einer großen Wirkung der steuerinduzierten Anreizeffekte auf die Wirtschaft im legalen und illegalen Bereich.

Wie bereits gezeigt, scheinen die steuerinduzierten Anreizmechanismen bzgl. des Faktorangebotsverhaltens nicht die von den Supply-Sidern erhofften Größenordnungen anzunehmen.

Damit muß auch der darauf basierende Zusammenhang zwischen Steuersatz und Steueraufkommen angezweifelt werden.

Desweiteren ist, wie bereits dargestellt, die Lage des Wendepunktes der LafferKurve für eine Volkswirtschaft entscheidend. Nur wenn sich die Volkswirtschaft in der prohibitiven Zone befindet, wirkt eine Steuersatzsenkung in Richtung Wirtschaftswachstum und Steuermehreinnahmen. Ist die Voraussetzung nicht gegeben, führen Steuersenkungen unweigerlich zu Einnahmeausfällen im Budget des Staates. Somit ist die Kenntnis über den genauen Kurvenverlauf und besonders über die Lage des prohibitive range überaus wichtig.

Nach Auffassung der Supply-Sider kann nicht in Frage gestellt werden, daß die Einnahmenhöhe einer Steuer negativ mit der Höhe ihres Steuersatzes korreliert sei. ${ }^{93}$ Durch empirische Daten können jedoch aber nur einzelne Punkte und lokale Informationen über die Steigung der Kurve ermittelt werden.

93 Vgl. Bartlett, B.: Reaganomics: supply-side economics in action, Westport 1981, S. 97 ff. 
Das Vorliegen des prohibitiven Falles kann in bestimmten Situationen erheblich differieren, wenn sich das Verhalten und die Anreize der Wirtschaftssubjekte aus nicht steuerlichen Gründen stark ändern. ${ }^{94}$

So können zum Beispiel in Kriegszeiten Steuersätze von über achtzig Prozent als nicht anreizschädlich betrachtet werden, da die Anreize nicht nur auf rational ökonomischen Überlegungen basieren.

Aber nicht nur außerökonomische Motive beeinflussen die Wirkung der Steuersatzvariationen, sondern auch andere gesamtwirtschaftliche Faktoren wie Arbeitslosenquote, Inflationsrate, Zinsraten, Haushaltsdefizite usw. führen zu jeweils unterschiedlichen Steuersatzeffekten. ${ }^{95}$

Insgesamt ergibt sich daraus, daß man selbst für einen eventuell genau meßbaren Kurvenabschnitt nicht genau sagen kann, ob er im prohibitiven oder im normalen Bereich liegt.

Einige Autoren erklären sogar einen Kurvenverlauf mit mehreren Maxima. ${ }^{96}$

Ein weiteres Problem der Kurvenbestimmung liegt im hohen Aggregationsgrad der Begriffe Grenzsteuersatz und Steuereinnahmen.

Die Zurechnung einer Steuereinnahme zu einem Steuersatz ist praktisch unmöglich, da die tatsächliche Einkommensteuer vielen Einflußfaktoren unterliegt und hinsichtlich des Steuergläubigers, der Steuerbemessungsgrundlage und ihrer Erhebungsstruktur differenziert ist. Ebenso müßte die Ermittlung des Grenzsteuersatzes die gesamte Tarifkonstruktion mit all ihren Progressivitäten, Linearitäten, Freibeträgen und Ausnahmen berücksichtigen. ${ }^{97}$ Die Ermittlung der marginalen Steuerquote muß über eine modellhafte Schätzung erfolgen, die dann in ihrer Aussagekraft unweigerlich problematisch zu interpretieren ist.

Aus dieser Vielzahl von Problemen und Ungenauigkeiten bei der Ermittlung und Konstruktion der Laffer-Kurve ergibt sich auch ihre kritisierte Irrealität.

Blinder kam in einer Analyse der Laffer-Kurve zu dem Ergebnis, daß die Annahme einer inversen Beziehung zwischen marginalem Steuersatz und Steuereinnahmen nicht haltbar sei. .98

94 Vgl. Wendisch, P.: Supply-Side Policy, Theoretische Grundlagen und prozeBpolitische Konsistenz, Frankfurt am Main 1984, S. 109.

95 Ebenda, S. 110.

96 Vgl. Henderson, D.: Limitations of the Laffer Curve as a Justification for Tax Cuts, in: Fink. R. (Hrsg.): Supply-Side Economics: A Critical Appraisal, Frederick, Maryland 1982, S. 225.

97 Vgl. Wendisch, P.: Supply-Side Policy, Theoretische Grundlagen und prozeßpolitische Konsistenz, Frankfurt am Main: Lang 1984, S. 110.

98 Vgl. Blinder, A. S., Thoughts on the Laffer Curve, in Meyer, L. A. (Hrsg.), The Supply-side Effects of Economic Policy, Boston 1981, S. 81-92 und Wendisch, P.: Supply-Side Policy. Theoretische Grundlagen und prozeßpolitische Konsistenz, Frankfurt am Main: Lang 1984, S. 123. 


\subsubsection{Die Trägheit angebotspolitischer Erfolge}

Die Angebotspolitik ist auf eine langfristige Verbesserung der Produktions- und Wachstumsbedingungen ausgerichtet.

Die Veränderung der Anreize, die letztlich zu einer vermehrten wirtschaftlichen Tätigkeit und zu Wirtschaftswachstum führen soll, benötigt sehr viel mehr Zeit, als die Wirkungsmechanismen der Nachfragepolitik, die durch zusätzliche öffentliche Ausgaben Investitionen und Arbeitsplätze schaffen kann.

Allein wegen dieser langfristigen Wirkungsmechanismen hat die Angebotspolitik einen großen „Attraktivitätsnachteil“ gegenüber der Nachfragepolitik. ${ }^{99}$

Die Angebotspolitik kann nicht, wie vielleicht die Nachfragepolitik, konkrete Versprechungen über kurzfristige ökonomische Auswirkungen verkünden, sondern sie ist auf eine allgemeine Verheißung angewiesen, daß die Kräfte des Marktes unter entsprechenden Voraussetzungen die gewünschten wirtschaftlichen Ergebnisse erreichen werden. 100

Damit stehen die Supply-Sider bei der wirtschaftspolitischen Durchsetzung ihrer Ideen unter dem starken Druck der jeweils bevorstehenden Wahlen. Die Angebotspolitiker stehen somit vor der Entscheidung, entweder ihrem Konzept treu zu bleiben und eine Wahlniederlage in Kauf zu nehmen, oder rechtzeitig nachfragepolitische Maßnahmen einzuflechten, um über ein wirtschaftliches Zwischenhoch die Wahlchancen zu verbessern. ${ }^{101}$

\subsubsection{Nachfrageeffekte einer angebotsorientierten Steuerpolitik}

Die Abbildung 9 zeigt ein wirtschaftliches Szenario, das die Supply-Sider nicht berücksichtigten.

Preisniveaustabilität kann in diesem Fall nur dann erreicht werden, wenn sich die Nachfrage, gemäß dem Sayschen-Gesetz, in gleichem Maß wie das gesamtwirtschaftliche Angebot entwickelt. Würde durch die Steuersenkungen „nur“ die gesamtwirtschaftliche Nachfrage positiv beeinflußt werden, könnte das Bruttoinlandsprodukt zwar erhöht werden, jedoch bei höheren Preisen (Punkt F). Diese Entwicklung erscheint nicht so abwegig, da durch eine Senkung der Einkommensteuer das verfügbare Einkommen steigt und damit möglicherweise ein höherer Konsum verbunden sein könnte. Aus einer auf eine Stärkung der Angebotsseite ausgerichtete Steuerpolitik könnte somit ungewollt eine Politik der keynesianischen Nachfragestärkung werden. ${ }^{102}$

$99 \mathrm{Vgl}$. Issing, O.:Supply-Side Economics, in Woll, A. (Hrsg.): Aktuelle Wege der Wirtschaftspolitik, Berlin 1983, S. 154.

100 Ebenda.

101 Ebenda, S. 155.

$102 \mathrm{Vgl}$. Economic Report of the President, 1994, S. 88. 


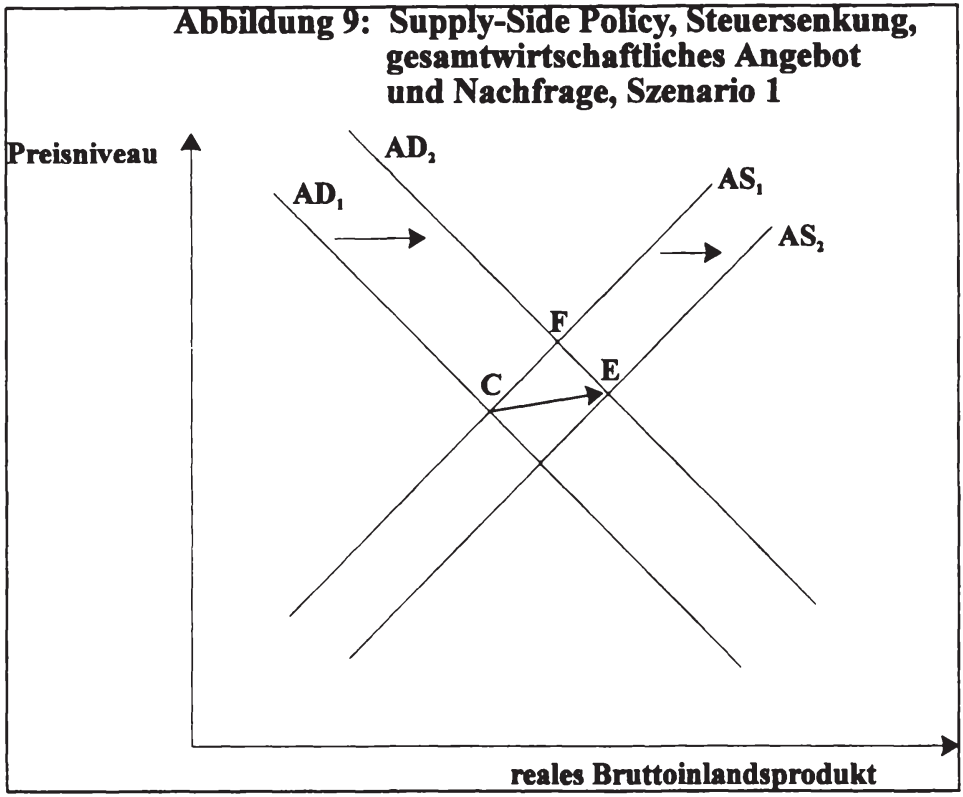

Quelle: Baumol, W. J., Blinder, A. S., Economics, Principles and Policy, sixth edition, 1994, S. 704, eigene Darstellung.

Treten durch die Steuersenkungen sowohl Angebots- als auch Nachfrageeffekte ein, kann in der graphischen Veranschaulichung der Punkt E erreicht werden. In diesem Fall jedoch bei einem etwas höheren Preisniveau als in der Ausgangssituation (Punkt C). Es zeigt sich, daß sich die Steuerung der Supply-Side Policy als schwierig erweist, wenn die Nachfrageeffekte unberücksichtigt bleiben.

Die Abbildung 10 zeigt ein weiteres wirtschaftliches Szenario, das deutlich macht, was passieren kann, wenn die Nachfrage in wesentlich stärkerem Maße als das gesamtwirtschaftliche Angebot auf Steuersenkungen reagiert.

Die Wirtschaft bewegt sich dann von der Ausgangssituation (Punkt C) zum Punkt G in der Graphik. Im Punkt $G$ ist ein höheres reales Bruttoinlandsprodukt erreicht, jedoch bei einem wesentlich höheren Preisniveau.

.... supply siders underestimate the effects of tax cuts on aggregate demand. If you cut personal taxes. individuals may possible work more, but they will certainly spend more."103

Es bleibt fraglich, ob die Wirtschaftssubjekte auf Steuersenkungen so reagieren, wie sich die Supply-Sider das vorstellen. Gegebenenfalls arbeiten sie dann mehr, mögli-

103 Vgl. Baumol, W. J., Blinder, A. S., Economics, Principles and Policy, sixth edition, 1994, S. 705. 
cherweise geben sie das zusätzliche Einkommen aber auch „nur“ aus. Selbst wenn alle, die von den Steuersenkungen profitieren, willig sind, mehr zu arbeiten, muß lange noch nicht gesagt sein, daß ihnen das auch möglich ist. Die meisten Arbeitnehmer haben stark kontraktbestimmte Arbeitszeiten (Arbeitsverträge), die es ihnen nicht erlauben, einfach mehr zu arbeiten, um damit ihr Einkommen weiter zu steigern, wenn von staatlicher Seite über eine Senkung der Grenzsteuersätze hierzu der ökonomische Anreiz gegeben würde. Die Vorstellungen der Supply-Sider über die Anreizwirkungen von Steuersenkungen treffen am ehesten auf die Wirtschaftssubjekte zu, die einer selbständigen Tätigkeit nachgehen und ihre Arbeitszeit weitgehend flexibel gestalten können. 104

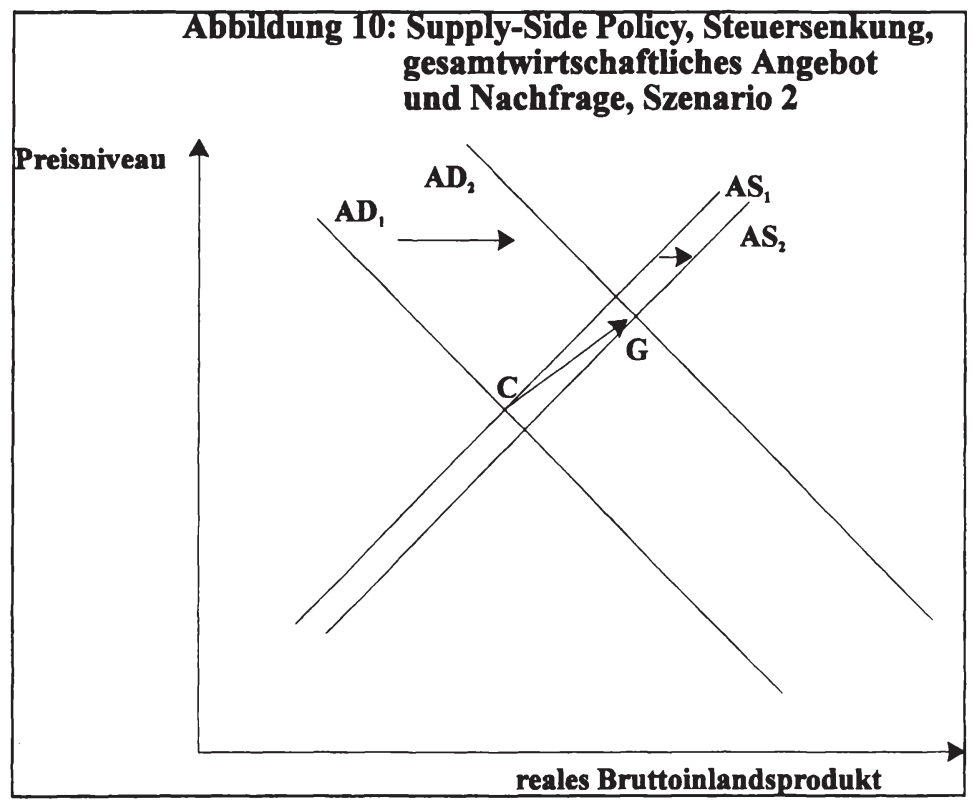

Quelle: Baumol, W. J., Blinder, A. S., Economics, Principles and Policy, sixth edition, 1994, S. 705, eigene Darstellung.

104 Vgl. Salant, W. S., A Critical Look at Supply-Side Theory and a Brief Look at some of its International Aspects, in: International Monetary Problems and Supply-Side Economics, Cohen, J. S. and Harcourt, G. C. (Hrsg.), London 1986, S. 108-124. 


\subsection{Resümee}

Die Suppy-Side Theorie ist, das zeigt das zweite Kapitel, keine neue Wirtschaftstheorie, die mit neuen Ideen versucht, wirtschaftliche Probleme anzugehen. Die Vertreter der angebotsorientierten Wirtschaftspolitik griffen die Überlegungen der klassischen Wirtschaftstheorie auf.

..The essential tenet of classical economic analysis is that people alter their behavior when economic incentives change. If the incentives for doing an activity increase relative to alternative activities, more of the attractive activity will be done." 105

Mit steuerlichen Anreizen die Leistungsbereitschaft der Menschen zu erhöhen erscheint einleuchtend, zumal diese Botschaft sich auch für Wahlkampfzwecke überaus erfolgreich einsetzen läßt. Dies hatte wohl auch Ronal Reagan erkannt und die SupplySide Policy zum Markenzeichen seiner Wirtschaftspolitik gemacht. Die sogenannte Reaganomics war damit geboren. Steuern senken und gleichzeitig die Einnahmen dabei erhöhen, erschien verlockend. Die Laffer-Kurve wurde zum Aushängeschild der Supply-Side Policy. Mit den Wirkungszusammenhängen, die sich aus der Laffer-Kurve ergaben, wurde politisch argumentiert. Die Steuerreformen, die Ronald Reagan während seiner zwei Amtszeiten durchführte, stehen in engem Zusammenhang mit der Laffer-Kurve. ${ }^{106}$

Die Laffer-Kurven-Argumentation der Supply-Sider erweist sich jedoch als überaus problematisch. Das Problem eines unzureichenden Wachstums der Wirtschaft maßgeblich auf ungenügende steuerliche Anreize zurückzuführen, erscheint nach der kritischen Analyse der Supply-Side Policy nicht überzeugend. Viele Fragen, die die Theorie der Supply-Side Policy aufwirft, bleiben unbeantwortet:

- Plausibilität und empirischer Gehalt der Laffer-Kurve,

- Steuerwirkungen auf den Arbeits- und Kapitalmarkt,

- Nachfrageeffekte einer angebotsorientierten Wirtschaftspolitik,

- Ungewisser Zeithorizont für die Auswirkungen einer angebotsorientierten Wirtschaftspolitik.

$\mathrm{Zu}$ Beginn der 80er Jahre wurde in den Vereingten Staaten der durchschnittliche Steuerzahler mit einem Grenzsteuersatz von etwa $32 \%$ belastet. ${ }^{107}$ Für diese Größenordnung erscheint es nicht besonders wahrscheinlich, daß die Vereinigten Staaten im prohibitiven Bereich der Laffer-Kurve lagen. Zumal für Schweden in einer Schätzung für das Jahr 1979 etwa ein marginaler Steuersatz von $83 \%$ ermittelt wurde; maximale Steuereinnahmen wären für dieses Jahr bei einem Grenzsteuersatz von $58 \%$ zu erzielen

$105 \mathrm{Vgl}$. Laffer, A. B., Supply-Side Economics, in: Financial Analysts Journal, September 1981, S. 32.

$106 \mathrm{Vgl}$. Kneer, J., Reaganomics-Elemente der amerikanischen Wirtschaftspolitik, in: Wirtschaftsdienst 1982/I, S. 30.

$107 \mathrm{Vgl}$. Barro, R. J., Sahasakul, C., Measuring the Average Marginal Tax Rate from the Individual Income Tax. The Journal of Business, vol. 56, no. 4, 1983, S. 419-452. 
gewesen. Diese Untersuchung legte folglich den Schluß nahe, daß Schweden zu diesem Zeitpunkt gleichsam auf dem negativ geneigten Abschnitt der Laffer-Kurve lag. ${ }^{108} \mathrm{Die}$ Supply-Sider blieben letztendlich den Beweis dafür schuldig, wie eine Senkung des Grenzsteuersatzes in den Vereinigten Staaten wirken würde. Die Existenz des LafferZusammenhangs wurde implizit angenommen und als politischer Hebel verwendet.

..Vor allem aber konnte die Doktrin der angebotsorientierten Wirtschaftspolitik das Entstehen eines Idealismus bewirken, der den gegenwärtig vorherrschenden Zynismus und Pessimismus überwand. ... Das neue Evangelium der von der Angebotsseite beherrschten Wirtschaft war für uns ein Hebel, mit dessen Hilfe sich eine Umkehr der herrschenden Tendenzen bewirken ließ, auch wenn das eine ungeheuer schwierige Aufgabe war."109

Die Analyse der Supply-Side Theorie zeigt, daß die Umsetzung der wirtschaftspolitischen Vorstellungen der Angebotsökonomen in den Vereinigten Staaten durch Ronald Reagan einem großen ökonomischen Experiment gleich kam, auf dessen Ergebnisse man nur gespannt sein konnte. Paul Krugman hat dies treffend zusammengefaßt:

..In any case. Ronald Reagan did exist, and he made supply-side economics the law of the land. How well did it work? “ 110

$108 \mathrm{Vgl}$. Feige. E. L., McGee. R. T., Sweden`s Laffer Curve: Taxation and the Unobserved Economy. Scandinavian Journal of Economics 85 (4), 1983, S. 499-519.

$109 \mathrm{Vgl}$. Stockman, D., Der Triumph der Politik. Die Krise der Reagan-Regierung und ihre Auswirkungen auf die Weltwirtschaft. München 1986, S. 52-53..

$110 \mathrm{Vgl}$. Krugmann, P.. Peddling Prosperity: eonomic sense and nonsense in the age of diminished expectations. New York-London 1994, S. 103. 


\section{Reaganomics}

Am 20. Januar 1981 übernahm Ronald Reagan die Amtsgeschäfte als 40. Präsident der Vereinigten Staaten; knapp einen Monat später, am 18. Februar, stellte er sein wirtschaftspolitisches Programm vor. Mit diesem Datum begann die Reagan-Revolution bzw. Reaganomics, die die Wirtschaftspolitik in den Vereinigten Staaten mehr als 10 Jahre bestimmen sollte. Im Jahr 1989 gab Reagan sein Amt an seinen Vizepräsidenten George Bush ab, der die Reaganomics in seinen wesentlichen Grundzügen fortsetzte. ${ }^{1}$

Mit der Wahl von Bill Clinton zum Präsidenten der Vereingten Staaten im November 1992 endete die Reaganomics. Dieser Zeitraum von 1981 bis 1992 stellt den Untersuchungszeitraum für das nachfolgende Kapitel dar. Es geht dabei weniger um die kurzfristigen Aspekte der angebotsorientierten Wirtschaftspolitik, sondern vielmehr darum, welche langfristigen Auswirkungen die neue Grundausrichtung der Wirtschaftspolitik auf die amerikanische Wirtschaft hatte.

\subsection{Das Reformprogramm}

Die Wirtschaftspolitik während der Präsidentschaft Ronald Reagans war von heftigen Auseinandersetzungen und Diskreditierungen begleitet. Die Reaganomics wurde von einigen Kritikern als „Voodoo-Economics“ abqualifiziert, weil sie vorausgesagt habe, die Steuersenkungen würden sich selbst finanzieren. ${ }^{2}$

Abgesehen von diesen Anfeindungen befand sich die U.S.A. in einer schwierigen wirtschaftlichen Ausgangsbasis zu Beginn der ersten Amtszeit Ronald Reagans im Jahr 1981. Ein Vergleich von wichtigen Wirtschaftsindikatoren des Jahres 1980 mit den 70er Jahren belegt dies.

Bruttoinlandsprodukt, Arbeitsproduktivität und Investitionen gingen im Jahr 1980 gegenüber dem Vorjahr zurück. Die Inflationsrate lag aufgrund der 2. Ölpeiskrise auf einem Rekordniveau von 13,5\%. Die hohe Arbeitslosigkeit und das hohe Staatsdefizit im Vergleich zu den 70er Jahren kennzeichneten weiterhin die wirtschaftliche Krise, in der sich die Vereinigten Staaten befanden.

Der Versuch einer konsequenten Umsetzung der Supply-Side Theorie in die ökonomische Praxis einer Volkswirtschaft wurde mit dem Amtsantritt von Ronald Reagan

1 Vgl. Kurz, R., Angebotsorientierte Wirtschaftspolitik in den USA: Grundlagen. Praxis und Konsequenzen. Tübingen 1993, S. 1-4.

2 Vgl. Roberts, P. C.. Angebotsorientierte Wirtschaftspolitik, theoretische Grundlagen und politische Erfolge: Eine Bewertung der amrikanischen Erfahrungen in den achtziger Jahren. Zeitschrift für Wirtschaftspolitik, Heft 1, 1989, S. 33. 
zum Präsidenten der Vereinigten Staaten im Jahr 1981 eingeleitet. Diese Grundausrichtung der amerikanischen Wirtschaftspolitik wurde - grosso modo - unter der Präsidentschaft Bush's bis zum Jahr 1992 beibehalten.

Tabelle 9: Die ïkonomische Ausgangssituation für Ronald Reagan im Jahr 1980 im Vergleich mit den 70er Jahren. (in \%)

\begin{tabular}{|l|c|c|}
\cline { 2 - 3 } \multicolumn{1}{c|}{} & $\mathbf{1 9 8 0}$ & $1970-1979$ \\
\hline Bruttoinlandsprodukt $^{1}$ & $\mathbf{- 0 , 5} \%$ & $2,9 \%$ \\
\hline Produktivität $^{\mathbf{2}}$ & $\mathbf{- 0 , 8} \%$ & $1,5 \%$ \\
\hline Investitionen $^{\mathbf{3}}$ & $\mathbf{- 2 , 5 \%}$ & $3,2 \%$ \\
\hline Inflation $^{\mathbf{4}}$ & $\mathbf{1 3 , 5 \%}$ & $7,1 \%$ \\
\hline Arbeitslosigkeit $^{\mathbf{5}}$ & $\mathbf{7 , 1 \%}$ & $6,2 \%$ \\
\hline Budgetdefizit $^{\mathbf{6}}$ & $\mathbf{2 , 2} \%$ & $1,7 \%$ \\
\hline
\end{tabular}

1: durchschnittliche jährliche Veränderungsrate des Bruttoinlandsprodukts in Preisen von 1987

2: durchschnittliche jährliche Veränderungsrate der Produktion pro Arbeitsstunde (Output per hour of all persons - Business sector)

3: durchschnittliche jährliche Veränderungsrate der Nettoinvestitionen außerhalb des Wohnungsbaus in Preisen von 1987 in Anteilen am Bruttoinlandsprodukt in Preisen von 1987

4: durchschnittliche jährliche Veränderungsrate des Konsumentenpreisindexes

5: durchschnittliche jährliche Veränderungsrate der Arbeitslosenquote

6: durchschnittliches jährliches Budgetdefizit des Bundeshaushaltes in Anteilen am Bruttoinlandsprodukt

Quelle: National Income and Product Accounts of the United States, Volume 2, 1959-88, U.S. Department of Commerce und Economic Report of the President, Februar 1994, eigene Berechnungen.

Das ökonomische Programm der Reagan-Administration, das der neu gewählte Präsident einen Monat nach seinem Amtsantritt dem amerikanischen Kongreß vorlegte, war von Beginn an auf eine Stärkung der ökonomischen Leistungsanreize ausgerichtet.

Am 18. Februar 1981 hat Ronald Reagan sein Wirtschaftsprogramm vorgestellt. Das Programm war mit dem Titel „A Program for Economic Recovery“ überschrieben. ${ }^{3}$ Wie schon in seiner Inaugarations-Rede am 20. Januar 1981 angekündigt, sollte das Wirtschaftsprogamm deutlich machen, daß die Rolle des Staates im Wirtschaftsprozeß in Zukunft eine andere sein sollte.

3 Vgl. The White House. Office of the Press Secretary: A Program for Economic Recovery, Washington D.C.. February 18, 1981. 
.In this present crisis, government is not the solution to our problem ${ }^{* 4}$

Der Staat sollte sich aus dem Wirtschaftsprozeß zurückziehen und simultan die steuerliche Belastung der Wirtschaftsakteure reduzieren.

Dies sollte im Rahmen des Wirtschaftsprogramms durch folgende Maßnahmen bewirkt werden:

- Kürzung der Einkommen- und Unternehmenssteuern

- Kürzung der Staatsausgaben und weitere Maßnahmen zur Reduzierung des Budgetdefizites

- Reduzierung der staatlichen Regulierungen für die Wirtschaft

- Eine neue Übereinkunft für eine stabile Geldpolitik

Durch diese Maßnahmen sollten folgende Effekte ausgelöst werden:

- Reduzierung der Inflationsrate

- Reduzierung der unproduktiven Belastungen der Wirtschaft durch die Bundesregierung, besonders aufgrund von staatlichen Regulierungen

- Reduzierung des Umfangs und der Rolle der Bundesregierung und ihre Einflußnahme auf wirtschaftliche Prozesse, die besser von Privatpersonen, Industrie, Bundesstaaten und Gemeinden übernommen werden können

- Reduzierung der Zinsen durch eine Reduzierung des Staatsdefizites

- Steigerung der Realeinkommen durch einen Anstieg der Investitionen und eine Ausweitung der Produktivität

In den Jahren 1981 bis 1986 wurde die amerikanische Steuergesetzgebung in mehreren Reformschritten umgestaltet. Die wesentlichen Reformen sind in der nachfolgenden Tabelle zusammengestellt und werden im Kapitel 3.2 detailliert beschrieben.

Tabelle 10: Die Steuerreformen während der Präsidentschaft Ronald Reagans

\begin{tabular}{|c|c|}
\hline Jahr & Steuerreform \\
\hline 1981 & The Economic Recovery Tax Act (ERTA) \\
\hline 1982 & $\begin{array}{c}\text { The Tax Equity and Fiscal Responsibility Act } \\
\text { (TEFRA) }\end{array}$ \\
\hline 1983 & Social Security Act (SSA) \\
\hline 1984 & The Deficit Reduction Act (DEFRA) \\
\hline 1986 & The Tax Reform Act (TRA) \\
\hline
\end{tabular}

Quelle: Akhtar, M. A.. Harris, E. S.. The Supply-Side Consequences of U.S. Fiscal Policy in the 1980s, in: Federal Reserve Bank of New York, Quarterly Review, Vol. 17. 1992. S. 17-18.

$4 \mathrm{Vgl}$. Reagan, R., First Inaugural Address, 20. Januar 1981, in: http://www.columbia.edu/acis/bartleby/inaugural/pres61.html, vom 12.09.1996. 
Die nächste Tabelle zeigt, wie durch die Steuerreformen in den Jahren 1982 bis 1988 die Höchststeuersätze der Einkommensteuer in den Vereinigten Staaten gesenkt wurden. Zu Beginn der Präsidentschaft Ronald Reagans lag der Spitzensteuersatz bei

$70 \%$; am Ende seiner zweiten Amtszeit bei 33 \%. Hinter der Senkung der Grenzsteuersätze verbarg sich die Theorie der Supply-Side Policy, die davon ausging, daß über eine Reduzierung der Steuerbelastung ein Anreiz für mehr Arbeit und Investitionen gesetzt werden würde..$^{5}$

Tabelle 11: Die maximalen Grenzsteuersätze der Einkommensteuer des Bundes in den Vereinigten Stacten. 1946 - 1992, (in \%)

\begin{tabular}{|c|c|}
\hline Jahre & Top-Grenzsteuersatz (\%) \\
\hline $1946-51$ & 91 \\
\hline $1952-53$ & 92 \\
\hline $1954-63$ & 91 \\
\hline 1964 & 77 \\
\hline $1965-67$ & 70 \\
\hline 1968 & 75,25 \\
\hline 1969 & 77 \\
\hline 1970 & 71,25 \\
\hline $1971-81$ & 70 \\
\hline $1982-86$ & 50 \\
\hline 1987 & 38 \\
\hline $1988-$ & 33 \\
\hline
\end{tabular}

Quelle: Tax Foundation. Facts and figures on government finance, Baltimore 1988, zitiert nach: Fullerton, D., Tax Policy, in: Feldstein, M. (Hrsg.). American Economic Policy in the 1980s, Chicago 1994, S. 168.

Für die Größenordnungen, in welchen sich die Wirtschaft und das Budget auf der Grundlage der neuen Gesetzgebungen in den nächsten Jahren entwickeln sollten, hatte Reagan konkrete Vorstellungen. Diese präsentierte er ebenfalls mit seinem Wirtschaftsprogramm im Jahr 1981.6 In Anteilen am Bruttosozialprodukt sollten die Staatseinnahmen nach der Steuerreform sinken. Der Rückgang von 21,1\% im Jahr 1981 auf 19,6\% im Jahr 1986 (Tabelle 12) resultiert jedoch aus dem erwarteten starken Anstieg des

5 Vgl. Fullerton, D.. Tax Policy, in: Feldstein, M. (Hrsg.), American Economic Policy in the 1980s, Chicago 1994, S. 167.

$6 \mathrm{Vgl}$. The White House. Office of the Press Secretary: A Program for Economic Recovery, Washington D.C.. February 18, 1981. 
nominalen Bruttosozialproduktes von 2.844 Mrd. Dollar im Jahr 1981 auf 4.806 Mrd. Dollar im Jahr 1986 (Tabelle 15). Über diese Projektion erklärt sich der Rückgang der geplanten Staatsausgaben in Anteilen am nominalen Bruttosozialprodukt von $23 \%$ im Jahr 1981 auf $19 \%$ im Jahr 1986 (Tabelle 14), obwohl die Staatsausgaben in diesem Zeitraum um 257,4 Mrd. Dollar steigen (Tabelle 13). Das starke Wachstum des Bruttosozialproduktes führt dazu, daß die Steuereinnahmen innerhalb von fünf Jahren um 341,9 Mrd. Dollar zunehmen (Tabelle 15). Da die Steuereinnahmen in diesem Zeitraum wesentlich stärker zunehmen als die Staatsausgaben (+ 257,4 Mrd. Dollar), wird im Rahmen des Reagan-Wirtschaftsprogramms ab dem Jahr 1984 ein Budgetüberschuß erzielt; im Jahr 1986 resultiert ein geplanter Haushaltsüberschuß von fast $30 \mathrm{Mrd}$. Dollar aus den wirtschaftspolitischen Maßnahmen (Tabelle 13).

Tabelle 12: Erwartete Staatseinnahmen auf der Basis der Steuerreform. in Anteilen des nominalen Bruttosozialprodukts (GNP). 1981 - 1986. (in \%)

\begin{tabular}{|l|c|c|c|c|c|c|}
\hline & $\mathbf{1 9 8 1}$ & $\mathbf{1 9 8 2}$ & $\mathbf{1 9 8 3}$ & $\mathbf{1 9 8 4}$ & $\mathbf{1 9 8 5}$ & $\mathbf{1 9 8 6}$ \\
\hline $\begin{array}{l}\text { Staatseinnahmen } \\
\text { vor der Steuerre- } \\
\text { form }\end{array}$ & 21,4 & 22,0 & 22,4 & 22,9 & 23,5 & 24,1 \\
\hline $\begin{array}{l}\text { Staatseinnahmen } \\
\text { nach der Steuerre } \\
\text { form }\end{array}$ & 21,1 & 20,4 & 19,7 & 19,3 & 19,3 & 19,6 \\
\hline
\end{tabular}

Quelle: The White House. Office of the Press Secretary: A Program for Economic Recovery. Washington D.C., Feb. 1981, S. 8.

Tabelle 13: Geplante Staatsausgaben und projizierter Budgetsaldo auf der Basis der Steuerreform. 1981 - 1986. in Mrd. Dollar

\begin{tabular}{|l|r|r|r|r|r|r|}
\hline & 1981 & $\mathbf{1 9 8 2}$ & $\mathbf{1 9 8 3}$ & $\mathbf{1 9 8 4}$ & $\mathbf{1 9 8 5}$ & $\mathbf{1 9 8 6}$ \\
\hline $\begin{array}{l}\text { geplante Staats- } \\
\text { ausgaben }\end{array}$ & 654,7 & 695,5 & 733,1 & 771,6 & 844,0 & 912,1 \\
\hline $\begin{array}{l}\text { projizierter } \\
\text { Budgetsaldo }\end{array}$ & $-54,4$ & $-45,0$ & $-22,9$ & $+0,5$ & $+6,9$ & $+29,9$ \\
\hline
\end{tabular}

Quelle: The White House, Office of the Press Secretary: A Program for Economic Recovery. Washington D.C., Feb. 1981, S. 2.

Die Projektion belegt, von welchen Annahmen Reagan bei der Abschätzung der Wirkungen seines Wirtschaftsprogramms ausging. Die Steuersenkungen kurbeln das Wirtschaftswachstum an und sorgen bis zum Ende des Jahres 1986 dafür, daß über zusätzliche Einnahmen, bei relativ geringer wachsenden Staatsausgaben, ein Budgetüber- 
schuß erzielt wird. Die geplanten Steuereinnahmen in Anteilen am Bruttosozialprodukt gehen zwar im Vergleich zu den prognostizierten Steuereinnahmen vor der Steuerreform zurück. Jedoch wächst im Rahmen der Projektion das erwartete Bruttosozialprodukt so stark, daß die prognostizierten Steuereinnahmen schon im Jahr 1984 absolut höher sind als die geplanten Staatsausgaben. Das Wirtschaftsprogramm von Ronald Reagan mit seinen geplanten Steuereinnahmen und Staatsausgaben bei gleichzeitig starkem Wirtschaftswachstum liefert einen entscheidenden Beweis dafür, daß das Konzept der Supply-Side Policy mit den Annahmen der Laffer-Kurve verwirklich werden sollte. Die umfassenden Steuersenkungen sollten sich spätestens im Jahr 1984 selbst finanzieren.

Tabelle 14: Enwartete Staatsausgaben auf der Basis der Steuerreform. in Anteilen des nominalen Bruttosozialprodukts (GNP), 1981 - 1986, (in \%)

\begin{tabular}{|l|c|c|c|c|c|c|}
\hline & $\mathbf{1 9 8 1}$ & $\mathbf{1 9 8 2}$ & $\mathbf{1 9 8 3}$ & $\mathbf{1 9 8 4}$ & $\mathbf{1 9 8 5}$ & $\mathbf{1 9 8 6}$ \\
\hline $\begin{array}{l}\text { geplante Staats- } \\
\text { ausgaben }\end{array}$ & 23,0 & 21,8 & 20,4 & 19,3 & 19,2 & 19,0 \\
\hline
\end{tabular}

Quelle: The White House, Office of the Press Secretary: A Program for Economic Recovery, Washington D.C., Feb. 1981, S. 2.

Tabelle 15: Envartete Statseinnahmen (incl. Beiträge zu den Sozialversicherungen) und erwartere Entwicklung des nominalen Bruttosozialprodukts (GNP) auf der Basis der Steuerreform. 1981. 1986. in Mrd. Dollar

\begin{tabular}{|l|c|c|c|c|c|c|}
\hline & $\mathbf{1 9 8 1}$ & $\mathbf{1 9 8 2}$ & $\mathbf{1 9 8 3}$ & $\mathbf{1 9 8 4}$ & $\mathbf{1 9 8 5}$ & $\mathbf{1 9 8 6}$ \\
\hline $\begin{array}{l}\text { erwartete } \\
\text { Staatseinnahmen }\end{array}$ & 600,2 & 650,5 & 710,2 & 772,1 & 850,9 & 942,1 \\
\hline erwartetes GNP & 2844,5 & 3188,7 & 3605,1 & 4000,5 & 4408,8 & 4806,6 \\
\hline
\end{tabular}

Quelle: The White House, Office of the Press Secretary: A Program for Economic Recovery, Washington D.C., Feb. 1981, S. 2 und eigene Berechnungen.

\subsection{Die Steuerreformen}

Die Steuerpolitik wurde von den Supply-Sidern zur zentralen Steuerungsgröße für die Finanzpolitik erklärt. Zielrichtung der Steuerpolitik war auf lange Frist gesehen ein stetiges Wirtschaftswachstum, das über Steuersenkungen erreicht werden sollte. ${ }^{7}$

7 Vgl. Akhtar, M.A., Harris, E.S.. The Supply-Side Consequence of U.S. Fiscal Policy in the 1980s, in: Federal Reserve Bank of New York, Quarterly Review, Vol. 17, Nr.1. 1992, S. 1. 
Weiterhin sollte auf lange Frist die Rolle des Staates in der Wirtschaft der Vereinigten Staaten kleiner und durch private Investitionen ersetzt werden. Dies kann, so die Vorstellungen von Reagan, nur über eine Kürzung der Staatsausgaben und Steuereinnahmen in Anteilen am Bruttosozialprodukt erreicht werden, was auch dazu führen würde, daß das Budgetdefizit reduziert würde. ${ }^{8}$

Die Steuerpolitik der Präsidentschaft Reagans wurde im wesentlichen durch zwei Steuergesetze bestimmt. Die Eckpfeiler (,cornerstones“y) seiner Steuerpolitik waren die Economic Recovery Tax Act (ERTA) im Jahre 1981 und die Tax Reform Act (TRA) im Jahre 1986. Die Jahre zwischen diesen umfassenden Änderungen der Steuergesetze wurden durch kleine Änderungen bei der Besteuerung begleitet. ${ }^{10}$

\subsubsection{Die Steuerreform von 1981 (ERTA)}

Die Economic Recovery Tax Act (ERTA), die am 1. Oktober 1981 in Kraft trat, sah im wesentlichen folgende Änderungen in der Steuergesetzgebung vor:

- Das Steuergesetz sah vor, daß bei der Bundeseinkommensteuer der Grenzsteuersatz der untersten Einkommensstufe von $13 \%$ auf $11 \%$ gesenkt und der Höchssteuersatz von $70 \%$ auf $50 \%$ reduziert wurde. Über den ganzen Tarif der Einkommensteuer sollte eine Senkung von ca. $25 \%$ erreicht werden.

- Dies erfolgte aus haushaltswirtschaftlichen Gründen in drei Schritten, die jeweils Steuersenkungen um $5 \%, 10 \%$ und $10 \%$ vorsahen. Zum 1. Juli 1983 war die Reduzierung der Bundeseinkommensteuer beendet.

- Die durchschnittliche Gesamtsteuerbelastung durch die Bundeseinkommensteuer wurde bis 1984 letztlich insgesamt um ca. $23 \%$ gesenkt. ${ }^{11}$

- Die Besteuerung von Veräußerungsgewinnen (capital gains tax) wurde von $28 \%$ auf $20 \%$ gesenkt.

- Ehepaare, deren gemeinsames Einkommen 30.000 Dollar überschritt, erhielten einen steuerlichen Freibetrag von $5 \%$ ihres Einkommens im Jahr 1982, in den darauffolgenden Jahren erhöhte sich der Freibetrag auf $10 \%$ ihres Einkommens.

- Zudem wurde die steuerliche Anrechenbarkeit der persönlichen Altersvorsorge ausgeweitetet. Ein Arbeitnehmer-Haushalt konnte nun 4.000 Dollar (Doppelverdiener) bzw. 2.250 Dollar (bei einem Verdienst) auf das zu versteuernde Einkommen anrechnen.

- Das Steuergesetz sah weiterhin vor, daß alle Steuerzahler ab dem Jahr 1985 einen steuerlichen Freibetrag von $15 \%$ aus ihren Zinseinnahmen erhalten. Die Obergrenze für diese Regelung wurde auf einen Freibetrag von 15.000 Dollar festgesetzt.

8 Vgl. Economic Report of the President, Februar 1982, S. 4 - 5.

$9 \mathrm{Vgl}$. Akhtar, M.A., Harris. E.S., The Supply-Side Consequence of U.S. Fiscal Policy in the 1980s. in: Federal Reserve Bank of New York, Quarterly Review, Vol. 17, Nr.1, 1992. S. 11.

10 Ebenda.

11 Ebenda. S. 17. 
- Mit der ERTA wurde festgelegt, daß ab 1985 eine Indexierung des Steuertarifs an die inflationäre Entwicklung erfolgt; diese Regelung schloß die steuerlichen Freibeträge und das steuerfreie Existenzminimum ein.

- Durch die Einführung des "Accelerated Cost Recovery System" wurde das bisherige Abschreibungssystem ersetzt. Es erlaubte den Unternehmen wesentlich kürzere Abschreibungszeiten im Bereich des Anlagevermögens. Maschinen konnten nun in einem Zeitraum von 5 Jahren statt bisher 8,6 Jahren steuerlich voll abgeschrieben werden. Autos konnten sogar in einem Zeitraum von 3 Jahren abgeschrieben werden. Bei Industrieanlagen verkürzte sich die Abschreibungszeit von 23,8 Jahren auf 15 Jahre.

Durch die steuerliche Entlastung der privaten Haushalte und Unternehmen erhoffte sich die Regierung für die folgenden Jahre ein Wirtschaftswachstum von 4 bis $5 \%$ sowie die Schaffung von 13 Millionen neuer Arbeitsplätze. ${ }^{12}$

Kritiker der Steuerreform warfen der Regierung vor, sie betreibe eine Politik gegen die Armen und würde im Gegenzug die Reichen begünstigen.

Wer bisher 100.000 Dollar Steuern zahlen mußte, erhielt in Folge der Steuersenkung eine Steuerentlastung von 25.000 Dollar. Im Gegensatz dazu verbesserte sich jemand, der bislang nur 1.000 Dollar Steuer zahlte lediglich um 250 Dollar. ${ }^{13}$

\subsubsection{Die Steuerreformen: TEFRA, SSA und DEFRA}

Die Erwartungen, daß allein aufgrund der massiven Steuersenkungen im Jahr 1981 ein starker wirtschaftlicher Aufschwung im darauffolgenden Jahr einsetzen würde, erwiesen sich als unzutreffend. Anstatt eines Konjunkturaufschwunges kam es zu einer schweren Rezession. Das Bruttosozialprodukt fiel im Jahr 1982 um 2,2 Prozent und die Arbeitslosenquote stieg auf 7.7 Prozent.

Zudem gingen die Staatseinnahmen zurück, während sich die Staatsausgaben aufgrund der Rezession stark erhöhten. Dadurch stieg das Haushaltsdefizit auf mehr als 100 Mrd. Dollar an.

Vor dem Hintergrund dieser wirtschaftlichen Entwicklung wurden im Sommer 1982 steuerliche Veränderungen beschlossen. Die „Tax Equity and Fiscal Responsibility Act (TEFRA)" hob zum Teil die Steuervergünstigungen des Vorjahres wieder auf. So wurden z.B. die Vorteile einer beschleunigten Abschreibung auf Anlagevermögen und die Möglichkeiten einer steuerlichen Anrechenbarkeit von Investitionen wieder eingeschränkt. Die individuellen Steuersätze blieben weitestgehend unangetastet. TEFRA war darauf ausgerichtet, die Unternehmen steuerlich stärker zu belasten, um dadurch höhere Steuereinnahmen zu erzielen. Für die kommenden drei Jahre erhoffte man sich

12 Vgl. Keller. K. J., Wirtschaftspolitik in den USA, Das wirtschaftspolitische Programm der Regierung Reagan und seine Hintergründe, in: WiSt Heft 5, Mai 1982, S. 230.

13 Vgl. Moore, T. G., Die Wirtschaftspolitik der Reagan-Administration, in: Vogel, O. (Hrsg.), Wirtschaftspolitik der achtziger Jahre. Köln 1982, S. 282. 
Mehreinnahmen von 100 Mrd. Dollar. ${ }^{14}$ Ein wesentlicher Bestandteil von TEFRA war eine $10 \%$ ige Quellensteuer auf Zins- und Dividendeneinnahmen, die jedoch aufgrund von öffentlichem Druck zum 1. Juli 1983 wieder aufgehoben wurde. ${ }^{15}$

Tabelle 16: Die wirtschaftliche Situation der Vereinigten Staaten in den Jahren 1981 und 1982

\begin{tabular}{|l|c|c|}
\cline { 2 - 3 } \multicolumn{1}{c|}{} & $\mathbf{1 9 8 1}$ & $\mathbf{1 9 8 2}$ \\
\hline Bruttoinlandsprodukt $^{1}$ & $+1,8 \%$ & $-2,2 \%$ \\
\hline Arbeitslosigkeit $^{2}$ & $7,1 \%$ & $7,7 \%$ \\
\hline Budgetdefizit $^{3}$ & $-30,3 \mathrm{Mrd} \$$ & $-109 \mathrm{Mrd} . \$$ \\
\hline Staatseinnahmen $^{4}$ & $639 \mathrm{Mrd} . \$$ & $635 \mathrm{Mrd} . \$$ \\
\hline Staatsausgaben $^{5}$ & $698 \mathrm{Mrd} . \$$ & $771 \mathrm{Mrd} . \$$ \\
\hline
\end{tabular}

1: prozentuale Veränderung des Bruttoinlandsprodukts im Vorjahresvergleich in Preisen von 1987

2: prozentuale Veränderung der Arbeitslosenrate im Vorjahresvergleich

3: Budgetdefizit des Bundes in Mrd. US-Dollar

4: Einnahmen des Bundes in Mrd. US-Dollar

5: Ausgaben des Bundes in Mrd. US-Dollar

Quelle: National Income and Product Accounts of the United States. Volume 2, 1959-88. U.S. Department of Commerce, eigene Berechnungen.

Die ..Social Security Act (SSA)“ des Jahres 1983 sah u.a. eine schrittweise Erhöhung der Sozialversicherungsbeiträge vor. Im Zeitraum von 1983 bis 1990 sollten die Beiträge zur Sozialversicherung, die jeweils zur Hälfte von Arbeitgeber und Arbeitnehmer bezahlt werden mußten, von 13,4 \% im Jahr 1983 auf 15,3\% im Jahr 1990 steigen. Dadurch sollten die Einnahmen erhöht werden, 1980 machten die Sozialversicherungsbeiträge $34 \%$ der Einnahmen des Bundeshaushaltes aus. ${ }^{16}$ Die Veränderungen der Steuergesetzte in den Jahren 1982 bis 1986 standen unter dem Eindruck der Budgetdefizite des Bundeshaushaltes (Tabelle 17). In den Jahren 1982,1983 und 1984 erreichte das Budegtdefizit jeweils Werte, die mehr als doppelt so hoch waren wie zu Beginn der Reaganomics im Jahr 1981. Die angebotsorientierte Wirtschaftspolitik schien nicht zu greifen.

Die „Defecit Reduction Act (DEFRA)“, die im Jahr 1984 beschlossen wurde, hatte ebenfalls zum Ziel, das Budgetdefizit zu verringern. Durch dieses Gesetz, das u.a die Abschreibungszeiten für Großprojekte von 15 Jahren auf 19 Jahre verlängerte, sollten Mehreinnahmen von 50 Mrd. Dollar in den Jahren 1984 bis 1987 erzielt werden.

$14 \mathrm{Vgl}$. Bartley. R. L.. The seven fat years - and how to do it again. New York 1995, S. 117 - 133.

15 Vgl. Müller-Oestreich, K., Der Einfluß der Finanzpolitik auf die Investitionsentwicklung in den USA unter Ronald Reagan. Berlin 1992, S. 75.

16 Ebenda. S. 76 
Gleichzeitig sah das Gesetz vor, daß die Ausgaben in diesem Zeitraum um 13 Mrd. Dollar gesenkt würden. ${ }^{17}$

Tabelle 17: Die Entwicklung des Budgetdefizites der Vereinigten Staaten in Relation zum Bruttoinlandsprodukt in den Jahren 1981-1984

\begin{tabular}{|l|c|c|c|c|}
\cline { 2 - 5 } \multicolumn{1}{c|}{} & $\mathbf{1 9 8 1}$ & $\mathbf{1 9 8 2}$ & $\mathbf{1 9 8 3}$ & $\mathbf{1 9 8 4}$ \\
\hline Budgetdefizit $^{1}$ & $1,9 \%$ & $4,3 \%$ & $5,3 \%$ & $4,4 \%$ \\
\hline
\end{tabular}

1: Budgetdefizit des Bundes in Anteilen am nominalen Bruttoinlandsprodukt

Quelle: National Income and Product Accounts of the United States, Volume 2, 1959-88, U.S. Department of Commerce, eigene Berechnungen.

Die Maßnahmen, die auf die Konsolidierung des Staatshaushaltes abzielten, erwiesen sich als nicht ausreichend. Der Anstieg der Staatsverschuldung konnte nicht aufgehalten werden. Das Budgetdefizit stieg weiter sprunghaft an und erreichte im Jahr 1986 eine Höhe von 201 Mrd. Dollar (Graphik 5).

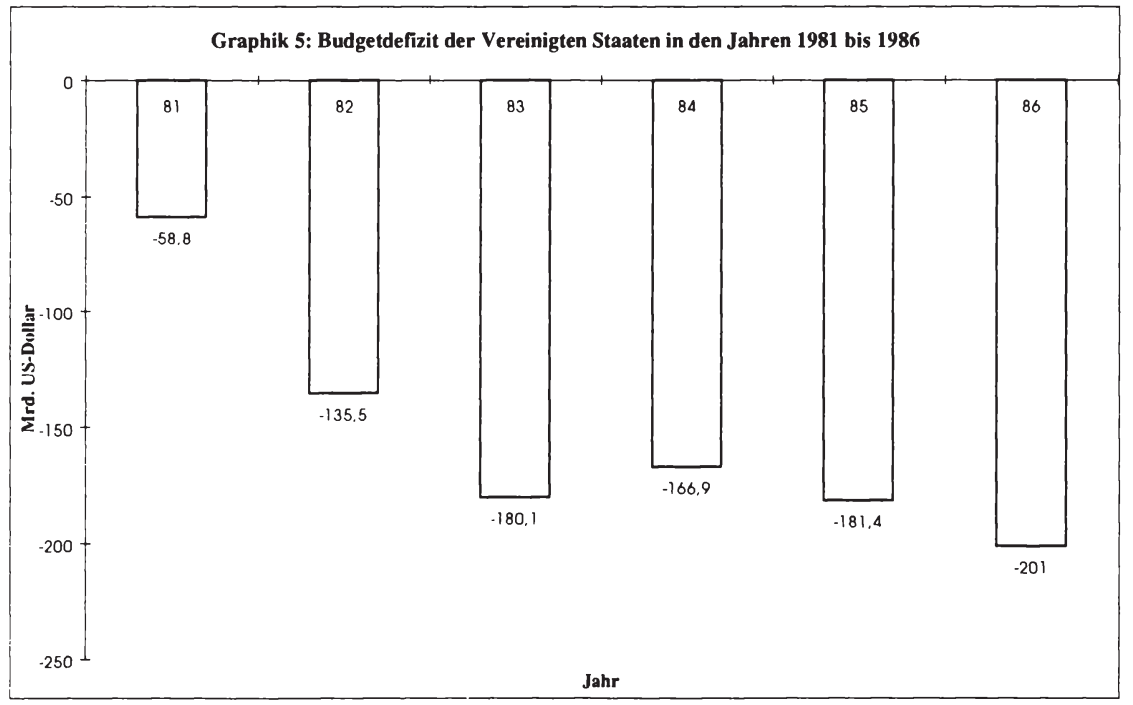

Quelle: National Income and Product Accounts of the United States, Volume 2, 1959-88, U.S. Department of Commerce, eigene Darstellung.

17 Ebenda. 


\subsubsection{Die Steuerreform von 1986 (TRA)}

Obwohl mit der Steuerreform 1981 bereits weitreichende Steuersenkungen sowohl im Bereich der Unternehmen als auch bei den Einkommensbeziehern in Kraft getreten sind, wurde das bestehende Steuersystem durch alle politischen Lager hindurch scharf kritisiert. Man warf dem Steuergesetz vor, es sei undurchschaubar, ungerecht und wachstumshemmend. Mitte des Jahres 1985 legte Reagan einen Vorschlag zur einfacheren, gerechteren und wachstumsfreundlicheren Gestaltung des Steuersystems vor. ${ }^{18}$

In Zusammenarbeit mit den Demokraten entwarf die Reagan-Administration eine umfassende Reform des amerikanischen Steuersystems, die unter dem Schlagwort "Fairness, Growth and Simplicity" stand. Im Oktober 1986 wurde die „Tax Reform Act (TRA)" verabschiedet.

Vor dem Hintergrund des großen Haushaltsdefizites sollten sich die Maßnahmen der Steuerreform allerdings aufkommensneutral verhalten. Um die Aufkommensneutralität zu erreichen, lautete die Kernforderung der Reform: Die Steuerbasis erweitern und die marginalen Steuersätze senken. ${ }^{19}$.

Die TRA kann als grundlegende Reform des Steuersystems und wahrscheinlich als die weitreichendste in der Geschichte der Vereinigten Staaten bezeichnet werden. ${ }^{20}$ Das Steuersystem sollte steuerpolitisch dereguliert werden und über die nochmalige Senkung der marginalen Steuersätze sollte die angebotsorientierte Wirtschaftspolitik fortgesetzt werden. Im Gegensatz zur Steuerreform von 1981 sollten die durchschnittlichen Steuersätze jedoch nahezu unverändert bleiben, um unveränderte Steuereinnahmen zu gewährleisten. ${ }^{21}$

Durch Beseitigung steuerlicher Schlupflöcher, Sonderregelungen und Subventionen sollte die Bemessungsgrundlage vergrößert und der Steuergerechtigkeit Rechnung getragen werden.22 Die Senkung der Steuersätze und die Reduzierung der Zahl der Steuerklassen sollten weitere Leistungsanreize schaffen und somit der Belebung der Wirtschaftstätigkeiten dienen.

Für den Zeitraum von 1986 bis 1991 wurde mit einer Steuerentlastung für die natürlichen Personen in Höhe von ca. 120 Mrd. Dollar gerechnet. Diesen Steuerverlust wollte die Regierung durch einen massiven Subventionsabbau zu Lasten der Kapitalgesellschaften ausgleichen. ${ }^{23}$

$18 \mathrm{Vgl}$. Kurz. R., Angebotsorientierte Wirtschaftspolitik in den USA: Grundlagen. Praxis und Konsequenzen, Tübingen 1993, S. 12-13.

19 Vgl. Rahmann, B., Napp. H., Die amerikanische Einkommensteuerreform als Vorbild für die Bundesrepublik Deutschland?, in: Diskussionspapiere zur Wirtschaftspolitik. Forschungsinstitut der Friedrich-Ebert-Stiftung, Nov. 1987, S. 4 und 11.

20 Vgl. Holtfrerich, C.-L., Wirtschaft USA, München 1991, S. 293.

21 Ebenda.

22 Vgl. Kurz, R., Angebotsorientierte Wirtschaftspolitik in den USA: Grundlagen. Praxis und Konsequenzen, Tübingen 1993, S. 12.

23 Vgl. Rahmann. B.. Napp. H., Die amerikanische Einkommensteuerreform als Vorbild für die Bundesrepublik Deutschland?, in: Diskussionspapiere zur Wirtschaftspolitik. Forschungsinstitut 
Die TRA trat 1988 in Kraft, für die Übergangsperiode 1987 galten fünf Steuerklassen mit Grenzsteuersätzen zwischen $11 \%$ und 38,5\%.24 ren: 25

Die wichtigsten Regelungen und Veränderungen der"Tax Reform Act (TRA)" wa- Reduzierung der Steuerklassen von fünfzehn auf drei.

- Senkung der Grenzsteuersätze auf maximal $33 \%$.

- Erhöhung des steuerfreien Grundfreibetrags auf 5.000 Dollar bei gleichzeitiger Indexierung.

- Abschaffung bzw. Einschränkung der steuerlichen Abzugsfähigkeit von Schuldzinsen auf Konsumentenkredite und Beiträge zur Altersvorsorge.

- Veräußerungsgewinne werden voll der Besteuerung unterworfen.

- Senkung des Körperschaftssteuersatzes auf $34 \%$.

- Einschränkung der Abschreibungserleichterungen.

- Die Absetzbarkeit von kommunalen und einzelstaatlichen Steuern, Zinszahlungen und Bewirtungskosten wird ebenfalls eingeschränkt.

- Die Investitionsförderung durch Steuergutschriften wird gestrichen.

Als Auswirkung dieser Reform wurde im Zeitraum von 1987 bis 1991 eine Entlastung bei der Einkommensteuer von 120 Mrd. Dollar bei entsprechenden Mehreinnahmen über die Körpeschaftssteuer erwartet. ${ }^{26}$

Die nachfolgende Auflistung zeigt die wichtigsten Regelungen der amerikanischen Einkommensteuerreform TRA in einem Vergleich mit dem bis dahin geltenden Steuerrecht. ${ }^{27}$

Das Ergebnis der amerikanischen Steuerreform von 1986 war erstaunlich. Die Steuergesetzänderungen der TRA waren wesentlich umfangreicher als bei der ersten großen Steuerreform während der Reagan-Ära im Jahr 1981.28 Die Vereinfachung des Einkommensteuersystems wurde durch das Gesetzeswerk erreicht. Die starke Umverteilung der Steuerlasten auf die Unternehmen ließ jedoch die Frage aufkommen, inwieweit sich diese zweite Steuerreform mit der angebotsorientierten wirtschaftspolitischen Strategie der Reaganomics vereinbaren läßt. Die steuerliche Entlastung der natürlichen Personen im Rahmen der TRA wäre als weiteres Indiz für eine konsequente angebotsorientierte Steuerpolitik zu werten. Demgegenüber steht jedoch die stärkere steuerliche Belastung der Kapitalgesellschaften. Die hohen Budgetdefizite erlaubten keine weiteren Rückgänge bei den Steuereinnahmen. Die Steuerreform mußte somit fast zwangsläufig

der Friedrich-Ebert-Stiftung, Nov. 1987, S. 12.

24 Vgl. Kurz, R., Angebotsorientierte Wirtschaftspolitik in den USA: Grundlagen, Praxis und Konsequenzen. Tübingen 1993, S. 13.

25 Ebenda.

26 Ebenda. S. 13-14.

27 Vgl. Rahmann, B., Napp, H., Die amerikanische Einkommensteuerreform als Vorbild für die Bundesrepublik Deutschland? in: Diskussionspapiere zur Wirtschaftspolitik, Forschungsinstitut der Friedrich-Ebert-Stiftung, Nov. 1987, S. 15-16.

$28 \mathrm{Vgl}$. Zschiegener H., Habert U., Einzelfragen der Übergangsregelungen der US-Steuerreform 1986. in: Internationale Wirtschaftsbriefe (IWB), Nr.12 vom 25.6. 1987, S. 391-398. 
aufkommensneutral konzipiert werden. In der ursprünglichen Projektion der Reaganomics war vorgesehen, daß die Staatsausgaben in Anteilen am Bruttoinlandsprodukt sinken würden. Auf dem Hintergrund der Steuerreform von 1986, die zwar weiterhin auf eine Senkung der marginalen Steuersätze setzte, aber die steuerliche Gesamtbelastung der amerikanischen Wirtschaft unverändert ließ, rückte die Frage in den Mittelpunkt, ob die angebotsorientierte Wirtschaftspolitik im Zusammenspiel von Steuersenkungen und Ausgabenkürzungen funktionierte.

\begin{tabular}{|c|c|c|}
\hline & ,altes" Steuerrecht & „Tax Reform Act (TRA)“ \\
\hline Einkommensteuersätze & $\begin{array}{l}15 \text { Tarifstufen } \\
\text { Eingangssteuersatz: } 11 \% \\
\text { Spitzensteuersatz: } 50 \%\end{array}$ & $\begin{array}{l}2 \text { bzw. } 3 \text { Tarifstufen } \\
1.15 \% \\
\text { 2. } 28 \% \\
\text { 3. Zuschlag von } 5 \% \text { auf } \\
\text { den Spitzensteuersatz für } \\
\text { ein Einkommen im Bereich } \\
\text { zwischen } 43.150 \$ \text { und } \\
89.560 \$ \text {, darüber gilt wie- } \\
\text { der der Steuersatz von } 28 \\
\% \text {. }\end{array}$ \\
\hline Freibeträge & $\begin{array}{l}1.080 \$ \text { je Haushaltsmit- } \\
\text { glied }\end{array}$ & $\begin{array}{l}\text { ab 1987: } 1.900 \$ \\
\text { ab 1988: } 1.950 \$ \\
\text { ab 1989: } 2.000 \$ \\
\text { ab 1990: Anpassung an die } \\
\text { Inflationsrate }\end{array}$ \\
\hline Pauschaler Abzugsbetrag & $\begin{array}{l}\text { für Ledige: } 2.480 \$ \\
\text { für Verheiratete: } 3.670 \$ \\
\text { für ledige Haushaltsvor- } \\
\text { stände: } 2.480 \$\end{array}$ & $\begin{array}{l}\text { für Ledige: } 3.000 \$ \\
\text { für Verheiratete: } 5.000 \$ \\
\text { für ledige Haushaltsvor- } \\
\text { stände: } 4.400 \$\end{array}$ \\
\hline
\end{tabular}




\begin{tabular}{|c|c|c|}
\hline & ,altes“ Steuerrecht & „Tax Reform Act (TRA)“ \\
\hline $\begin{array}{l}\text { Doppelverdiener- } \\
\text { Freibetrag }\end{array}$ & $3.000 \$$ & aufgehoben \\
\hline $\begin{array}{l}\text { Bundesstaaten- und Ge- } \\
\text { meindesteuern }\end{array}$ & $\begin{array}{l}\text { abzugsfähig: Einkommen- } \\
\text { steuer, Vermögensteuer, } \\
\text { Kaufsteuer }\end{array}$ & $\begin{array}{l}\text { abzugsfähig: Einkommen- } \\
\text { steuer, Vermögensteuer }\end{array}$ \\
\hline Hypothekenzinsen & unbegrenzt abzugsfähig & für 1. und 2. Haus absetzbar \\
\hline Kreditkosten & absetzbar & nicht absetzbar \\
\hline & ,altes" Steuerrecht & „Tax Reform Act (TRA)“ \\
\hline $\begin{array}{l}\text { Kosten ärztlicher Behand- } \\
\text { lung }\end{array}$ & $\begin{array}{l}\text { zumutbare Eigenbelastung } \\
\text { von } 5 \% \text { des steuerlichen } \\
\text { Bruttoeinkommens, darüber } \\
\text { hinausgehende Kosten sind } \\
\text { abzugsfähig }\end{array}$ & $\begin{array}{l}\text { zumutbare Eigenbelastung } \\
\text { von 7,5\% des steuerlichen } \\
\text { Bruttoeinkommens, darüber } \\
\text { hinausgehende Kosten sind } \\
\text { abzugsfähig }\end{array}$ \\
\hline Rentenversorgung & $\begin{array}{l}\text { Abzugsfähigkeit von Bei- } \\
\text { trägen zur privaten Alters- } \\
\text { vorsorge bis zu } \\
2.250 \$ \text { im Jahr }\end{array}$ & $\begin{array}{l}\text { Die Abzugsfähigkeit bis zu } \\
2.250 \$ \text { für Personen mit ei- } \\
\text { nem Einkommen bis zu } \\
25.000 \$ \text { (Ledige) bzw. } \\
40.000 \$ \text { (Verheiratete) } \\
\text { bleibt erhalten. Darüber } \\
\text { hinaus reduziert sich die } \\
\text { Abzugsfähigkeit, bei einem } \\
\text { Einkommen von } 35.000 \$ \\
\text { (Ledige) bzw. } 50.000 \$ \\
\text { (Verheiratete) entfällt die } \\
\text { Abzugsfähigkeit }\end{array}$ \\
\hline
\end{tabular}




\begin{tabular}{|l|l|l|}
\hline $\begin{array}{l}\text { Körperschaftssteuer - } \\
\text { höchster Steuersatz }\end{array}$ & ,altes "Steuerrecht & "Tax Reform Act (TRA)“ \\
\hline $\begin{array}{l}\text { Veräußerungsgewinne } \\
\text { versteuerbar mit: }\end{array}$ & $28 \%$ & $34 \%$ \\
\hline Forschungs- und Entwick- & $25 \%$ sind absetzbar & $\begin{array}{l}\text { werden wie normales Ein- } \\
\text { kommen (Gewinne) besteu- } \\
\text { ert }\end{array}$ \\
\hline $\begin{array}{l}\text { Abschreibungen } \\
\text { lungskosten }\end{array}$ & beschleunigte Abschreibung \\
möglich & $\begin{array}{l}\text { Erhöhung der Abschrei- } \\
\text { bungsklassen von bisher 6 } \\
\text { auf 8 Kategorien. Insbeson- } \\
\text { dere bei Immobilien wurde } \\
\text { die Abschreibungsdauer } \\
\text { von 19 Jahren auf 27,5 Jah- } \\
\text { re erhöht. }\end{array}$ \\
\hline Investitionssteuerkredit & $10 \%$ & $\begin{array}{l}\text { Zum 1. Januar 1986 rück- } \\
\text { wirkend aufgehoben }\end{array}$ \\
\hline
\end{tabular}

Quelle: Rahmann, B., Napp. H., Die amerikanische Einkommensteuerreform als Vorbild für die Bundesrepublik Deutschland?, in: Diskussionspapiere zur Wirtschaftspolitik. Forschungsinstitut der Friedrich-Ebert-Stiftung, Nov. 1987, S. 15-16.

\subsection{Staatsausgaben}

Die Gestaltung der Finanzpolitik unter Ronald Reagan stand zu Beginn seiner ersten Amtszeit unter seiner vorgegebenen politischen Maßgabe „A Stronger Nationale Defense within Restrained Overall Spending Levels" ${ }^{29}$. Das Budget sollte so umgestaltet werden, da $B$ der Verteidigungshaushalt massiv gestärkt und zivile Ausgaben deswegen gekürzt werden sollten. Die Verteidigungsausgaben sollten auf einen Anteil an den Ge-

$29 \mathrm{Vgl}$. The White House, Office of the Press Secretary: A Program for Economic Recovery, Washington D.C., February 18, 1981, S. 5. 
samtausgaben des Bundes von 24,1\% im Jahr 1981 auf 32,4 \% bis zum Jahr 1984 steigen. ${ }^{30}$ Damit die Umstrukturierung des Haushaltes nicht zu zusätzlichen Haushaltsdefiziten führen würde, war eine Begrenzung der Ausgabenseite für bestimmte Bereiche erforderlich. ${ }^{31}$

Insgesamt sollte die Staatsausgabenquote aber dennoch sinken. Das Wirtschaftsprogramm von Reagan aus dem Jahr 1981 sah vor, daß der Anteil der Staatsausgaben am nominalen Bruttoninlandsprodukt von 23 \% im Jahr 1981 auf $19 \%$ im Jahr 1986 sinken sollte. 32 Die Wachstumsrate der Staatsausgaben sollte unter der Zunahme der Staatseinnahmen und des Bruttoinlandsprodukts liegen.

Im Rahmen der „Omnibus Budget Reconciliation Act (OBRA)“ vom August 1981 wurden die Sparmaßnahmen beschlossen. Maßgeblich waren hiervon die Sozialausgaben und die Bundeszuschüsse (Grants in Aid) betroffen. Die Kürzungsbeschlüsse sollten während der Haushaltsjahre 1981 bis 1984 das Budget um insgesamt 130,6 Mrd. Dollar entlasten. ${ }^{33}$

Die Erhöhung der Militärausgaben zu Lasten der Sozialleistungen wurde mit der wachstums- und leistungshemmenden Wirkung der Sozialausgaben und mit dem militärischen Ungleichgewicht zur Sowjetunion und der daraus resultierenden Notwendigkeit zur Stärkung des amerikanischen Militärpotentials begründet. Zudem sahen die Angebotspolitiker in der forcierten Entwicklung militärischer Technologie und der Expansion der Rüstungsindustrie einen Investitions- und Innovationsschub für die gesamte Wirtschaft. ${ }^{34}$

Im Rahmen der Kürzungen der Sozialleistungen wurden u.a. die Einkommensgrenzen für die Bezugsberechtigung von Sozialhilfegeldern stark reduziert. Weiterhin wurden das Arbeitsbeschaffungsprogramm für jugendliche Arbeitslose sowie die Studienförderung auf Darlehensbasis abgeschafft. Die Regierung schloß das Community Services Administration Bureau, das in den sechziger Jahren zur Bekämpfung der Armut auf lokaler Ebene gegründet worden war. Die Ausgabenkürzungen sahen zudem vor, daß die Arbeitsplätze des öffentlichen Dienstes nicht mehr subventioniert werden sollten; des weiteren einen Abbau von 75.000 staatlichen Arbeitsplätzen in einem Zeitraum von 3 Jahren.

Die Kürzungen bei den Finanzzuweisungen an die Bundesstaaten wurden damit begründet, daß die Zuweisungen nicht mehr zweckgebunden erfolgen sollten, sondern en

30 Ebenda, S. 6.

31 Vgl. Roberts. P. C.. Supply-Side Economics: An Assesment of The American Experience In The 1980s, February 1989, National Westminster Bank. Quarterly Review, S. 65-68.

32 Vgl. The White House, Office of the Press Secretary: A Program for Economic Recovery, Washington D.C., February 18, 1981, S. 2.

33 Vgl. Müller-Oestreich, K., Der Einfluß der Finanzpolitik auf die Investitionsentwicklung in den USA unter Ronald Reagan, Berlin 1992, S. 72-73.

$34 \mathrm{Vgl}$. Schaal, P., Reaganomics - Konzept und Perspektiven der neuen amerikanischen Wirtschaftspolitik. List-Forum. Band 11 (1982), S. 396-397. 
bloc gewährt wurden. Außerdem sollte den Bundesstaaten ein größeres Mitspracherecht bei gemeinsamen Vorhaben eingeräumt werden. ${ }^{35}$

Die Kürzungen im Sozialetat betrafen vornehmlich die Wohlfahrtsprogramme und damit die Leistungen für die einkommensschwachen Gruppen der amerikanischen $\mathrm{Ge}$ sellschaft. Gerade diese Ausrichtung der Finanzpolitik entsprach der sozialpolitischen Intention der Supply-Side Policy, bei den Leistungen des Staates sollte die Leistungsfähigkeit des Individuums in den Mittelpunkt rücken. Wer wenig leistet, kann vom Staat auch wenig erwarten. Wer mehr leistet, bekommt dies auch vom Staat über geringere Steuern honoriert.

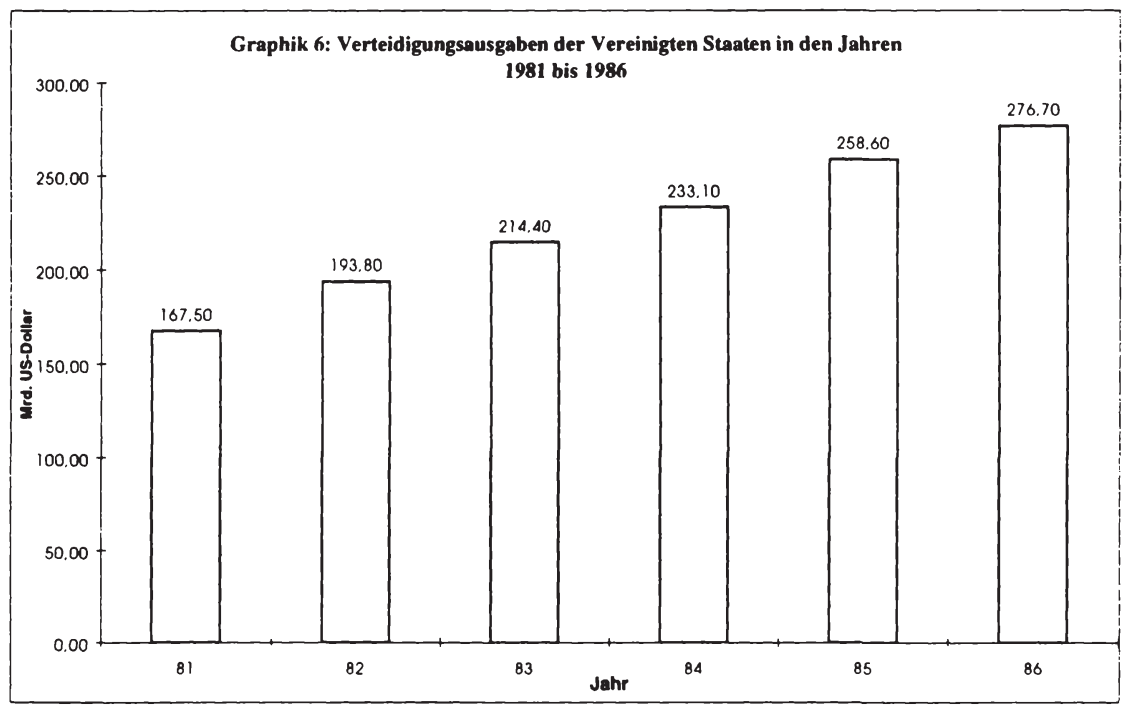

Quelle: National Income and Product Accounts of the United States, Volume 2. 1959-88, U.S. Department of Commerce, eigene Berechnungen und Darstellung.

Die Graphik 6 zeigt die Entwicklung der Verteidigungsausgaben von 1981 bis 1986. In diesem Zeitraum sind diese um $110 \mathrm{Mrd}$. Dollar gestiegen. Mit diesem Anstieg hat sich eine Veränderung der Haushaltsstruktur des Bundes ergeben. Die Graphik 7 zeigt einen Vergleich der Haushaltsstrukturen zwischen den Jahren 1981 und 1986. Die Verteidigungsausgaben sind in Anteilen am Bundeshaushalt von $24 \%$ auf $26,9 \%$ gestiegen, die Sozialausgaben sind im gleichen Zeitraum von $41,1 \%$ auf $38,8 \%$ zurückgegangen. Die Strukturveränderungen des Haushaltes bestätigen bei den Sozialausgaben

35 Vgl. Keller, K., Wirtschaftspolitik in den USA, Das wirtschaftspolitische Progamm der Regierung Reagan und seine Hintergründe, in: WiSt, Heft 5, Mai 1982, S. 230. 
die ursprüngliche Zielsetzung von Ronald Reagan, die Erhöhung des Verteidigungshaushaltes über eine Senkung der Sozialausgaben und der Bundeszuschüsse zu finanzieren. Die Bundeszuschüsse sind in diesem Zeitraum von

$12,6 \%$ auf $10,5 \%$ zurückgegangen.

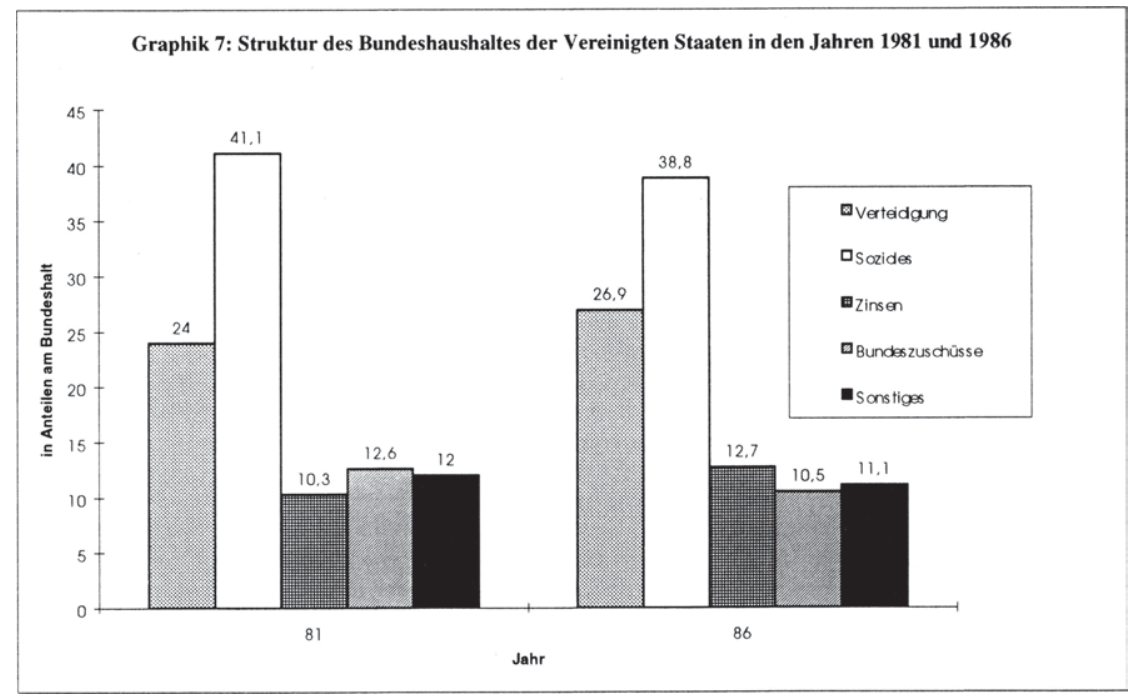

Quelle: National Income and Product Accounts of the United States, Volume 2, 1959-88, U.S. Department of Commerce, eigene Berechnungen und Darstellung.

Weiterhin zeigt die Graphik 7, daß die Budgetdefizite den Bundeshaushalt stark belasteten. Von 1981 bis 1986 ist der Anteil der Zinsausgaben am Bundeshaushalt von $10,3 \%$ auf $12,7 \%$ gestiegen. Im Jahr 1981 wurden $71,7 \mathrm{Mrd}$. Dollar für die Staatschulden bezahlt , fünf Jahre später mußten bereits $131 \mathrm{Mrd}$. Dollar für Zinszahlungen aufgewendet werden. ${ }^{36}$

Die Probleme, die sich aus dem Budgetdefizit ergaben, entwickelten sich für Ronald Reagan zu Beginn seiner zweiten Amtszeit zum wirtschaftspolitischen Dilemma. Das Budgetdefizit gefährdete immer mehr die angebotsorientierte Grundausrichtung seiner Wirtschaftspolitik.

Im Jahr 1985 initiierte der Präsident zusammen mit seiner republikanischen Mehrheit im Kongreß Sparmaßnahmen, die über eine Neuregelung des Haushaltsbegleitverfah-

$36 \mathrm{Vgl}$. National Income and Product Accounts of the United States, Volume 2, 1959-88, U.S. Department of Commerce, New York 1991. 
rens gewährleistet werden sollten. Die „Emergency Deficit Control and Balanced Budget Act", auch bekannt unter der Bezeichnung Gramm-Rudmann-Holling Act, sah vor, da $\beta$ genaue Höchstgrenzen für die Defizite des Bundes für die nächsten Jahre festgelegt werden sollten. Bis zum Jahr 1991 sollte die Nettoneuverschuldung des Bundes völlig abgebaut werden. Um dies sicherzustellen, wurde für den Fall, daß sich Präsident und Kongreß nicht auf Maßnahmen zum Abbau des Haushaltsdefzites einigen könnten, ein automatischer Ausgabenkürzungsmechanismus festgelegt. Die Kürzungen sollten dann bei allen Bewilligungsposten, und zwar je zur Hälfte im Verteidigungsbudget und bei den Ausgaben für zivile Zwecke, vorgenommen werden.

Es zeigte sich jedoch sehr bald, daß dieses Gesetz sein Ziel verfehlte. Über den Verkauf von staatlichen Vermögenswerten und die zeitliche Verschiebung von Gehaltszahlungen an Bundesangestellte von einem Rechnungsjahr in das andere versuchte man, das Defizit zwar kurzfristig zu drücken, auf lange Sicht brachten diese Maßnahmen jedoch keine Einsparungen. Im September 1987 wurde die Gramm-Rudmann-Holling Act vom Kongreß geändert. Die neue Gesetzgebung paßte die Defizitziele an und verschob den Haushaltsausgleich um zwei Jahre auf 1993.37 Das Gesetzgebungsverfahren zur Reduzierung des amerikanischen Bugetdefizites zeigte letztendlich, daß die politischen Institutionen trotz des immensen Haushaltsdefizites, nicht willens waren, die Staatsausgaben nachhaltig zu senken. ${ }^{38}$

\subsection{Deregulierungen}

In seinem Wirtschaftsprogramm „A Program for Economic Recovery“ erklärte Ronald Reagan, daß staatliche Regulierungen den Wirtschaftsprozeß erheblich beeinträchtigen und die Industrie und Verbraucher mit zusätzlichen Kosten belasten würden. ${ }^{39}$

Am 22. Januar 1981 kündigte Ronald Reagan die sogenannte "Presidential Task Force on Regulatory Relief" an, die unter dem Vorsitz des damaligen Vizepräsidenten George Bush stehen sollte. ${ }^{40}$

Diese Kommission hatte die Aufgabe, alle bestehenden Regulierungsvorschriften auf ihre Notwendigkeit hin zu überprüfen und eine Regulierungsreform auszuarbeiten.

Weiterhin wurde entschieden, alle behördlichen Vorschriften nach ihrer Wichtigkeit einzustufen und anhand einer Kosten-Nutzen-Analyse auf ihre Effektivität und ihre Kosten hin zu untersuchen. ${ }^{41}$

$37 \mathrm{Vgl}$. Horst, P., Haushaltspolitik und Regierungspraxis in den USA und der Bundesrepublik Deutschland: ein Vergleich des haushaltspolitischen Entscheidungsprozesses beider Bundesrepubliken zu Zeiten der konservativen Regierung Reagan/Bush (1981 - 92) und Kohl (1982 - 93), Frankfurt am Main 1995, S. 93-98.

$38 \mathrm{Vgl}$. DIE ZEIT, Angriff auf die Armen, Nr. 21, vom 19. Mai 1995, S. 21-22,

$39 \mathrm{Vgl}$. The White House, Office of the Press Secretary: A Program for Economic Recovery, Washington D.C., February 18, 1981, S. 1-2.

$40 \mathrm{Vgl}$. The White House, Office of the Press Secretary: President Reagan's Initiatives to Reduce Regulatory Burdens, Washington D.C., February 18, 1981, S. 1. 
Mit dem geplanten Abbau von Regulierungen während der Präsidentschaft Ronald Reagans wird wiederum die Angebotsorientierung seiner Wirtschaftspolitik deutlich. Der Einfluß des Staates auf die Wirtschaft soll verringert werden, um die natürlichen Kräfte eines freien Marktes zu stärken. Dieser Wirtschaftspolitik liegt die Auffassung zugrunde, daß staatliche Regulierungen die bestmögliche Allokation der Ressourcen verhindern. Die Supply-Sider waren der Auffassung, daß die sich ausweitende staatliche Bürokratie maßgeblich an den wirtschaftlichen Problemen der Vereinigten Staaten in den 70er Jahren verantwortlich war. Die Ausweitung des Staatssektors hat nach ihrer Einschätzung dazu beigetragen, da $ß$ zunehmend nicht profitable staatliche Investitionen über Staatsschuldenpapiere finanziert wurden. Die höhere Staatsverschuldung habe in Folge der zunehmenden Staatsausgaben zu höheren Kapitalmarktzinsen und zu einer Verdrängung der privaten Investitionen geführt, so die crowding out Hypothese der Supply-Sider. ${ }^{42}$

Die Sonderkommission, die die staatlichen Regulierungen reformieren sollte, bekam vom Präsidenten folgende Aufgaben übertragen: ${ }^{43}$

- Zusammenstellung von staatlichen Regulierungen, die reformbedürftig sind.

- Aufdeckung von Regulierungen von staatlichen Behörden, die sich gegenseitig behindern und zu Kompetenzüberschneidungen führen.

- Auflistung von Regulierungen, die besonders die Privatwirtschaft behindern.

- Einführung einer Kosten-/Nutzenanalyse für neue Regulierungen.

- Einschnitte in die Etats von Behörden, die für Regulierungen zuständig sind.

- Sensibilisierung des öffentlichen Bewußtseins für staatliche Regulierungen, die die Staatsausgaben nicht erhöhen.

- Empfehlungen an den Präsidenten bei der Berufung von deregulierungsbereiten Leitern staatlicher Behörden.

Die aufgelisteten Aufgaben der „Task Force on Regulatory Relief“ waren sehr allgemein gehalten. Die Regulierungsbestrebungen, die in den 80er Jahren in den Vereinigten Staaten verstärkt unternommen wurden, setzten schon vor Ronald Reagan Ende der 70er Jahre in den U.S.A. ein. Bereits 1978 wurde mit der „Airline Deregulation Act" die Deregulierung im Luftverkehr beschlossen, die im Jahr 1984 erfolgreich abgeschlossen wurde. Weitere Deregulierungen im Bereich des Straßengüterverkehrs, Schienenverkehrs und Finanzbereichs lagen vor der Amtszeit Ronald Reagans. Die Reaganomics war nicht die politische Wende für die Deregulierungen, diese wurde in den Jahren zuvor eingeleitet. Eher bekamen die Deregulierungen einen anderen ökonomischen Aspekt. In den Jahren zuvor stand die Preisargumentation im Mittelpunkt der politischen Diskussion. Deregulierungen sollten mehr Preisflexibilität durch ver-

41 Vgl. Weidenbaum, M., Regulatory Reform: A Fast Start, in: Economic Impact, 1981/4, S. 10.

42 Vgl. Gilder, G., The Supply-Side, in: Fink, R. H. (Hrsg.), Supply-Side Economics: A Critical Appraisal, Maryland 1982, S. 29-31.

$43 \mathrm{Vgl}$. The White House. Office of the Press Secretary: President Reagan's Initiatives to Reduce Regulatory Burdens, Washington D.C., February 18, 1981, S. 2. 
stärkten Wettbewerb erzeugen. Die Reaganomics setzte den Schwerpunkt auf Wachstumsimpulse für die Wirtschaft. ${ }^{44}$

Die wichtigsten Deregulierungen in der Zeit der Reaganomics sind im nachfolgenden Überblick zusammengestellt: 45

Im Bereich des Verkehrssektors wurde mit der „Bus Regulatory Reform Act“ des Jahres 1982 der Intercity-Busverkehr weitgehend privatisiert. Die gesamtwirtschaftlichen Vorteile der Deregulierung im gesamten Straßenverkehr wurden auf 40-60 Mrd. Dollar geschätzt.

- Die Deregulierung des Ölmarktes wurde von der Reagan-Regierung erreicht. Die Preiskontrollen für im Inland gefördertes Öl wurden aufgehoben.

- Der Sektor der Telekommunikation wurde dereguliert. Das Monopol von AT\&T wurde 1982 aufgehoben. Als Ergebnis ergaben sich für den Verbraucher eine größere Produktvielfalt und Preisdifferenzierung.

- Die Arbeitsplatzsicherheit versuchte man durch Deregulierungen zu verbessern. Unternehmesinspektionen ersetzen die bisherige Praxis von Buchprüfungen.

- Die Pharmaindustrie wurde durch verkürzte Zulassungsverfahren bei neuen Medikamenten und Exporterleichterungen bei in den U.S.A. noch nicht zugelassenen Arzneimitteln gestärkt.

- In der ,Executive Order 12991“ des Präsidenten vom 17. Februar 1981 wurden die Behörden zu einer Kosten-Nutzen-Analyse bei Regulierungsvorhaben verpflichtet. $\mathrm{Zu}$ Beginn seiner zweiten Amtszeit wurde mit der „Executive Order 12498“ im Januar 1985 die Überwachung der Regulierungsaktivitäten stärker kontrolliert. Diese Bestimmung sah vor, daß das Regulierungsprogramm der staatlichen Behörden veröffentlicht werden mußte.

Die Reaganomics hat durch ihre Deregulierungsbemühungen zu einer Markt- und Preistransparenz und Flexibilisierung in wichtigen Breichen der Wirtschaft, maßgeblich im Verkehrssektor, beigetragen. Die Verwaltungspraxis zur Entlastung der Wirtschaft wurde flexibler, was nicht in allen Bereichen, z.B. im Umweltschutz, Verbesserungen brachte. In der Umweltpolitik gelang es erst 1990, aufgrund der politischen Maßgabe für die Deregulierung der Wirtschaft, Gesetzesvorhaben zur Reinhaltung der Luft durchzusetzen. 46

Die ökonomische Beurteilung der Deregulierungen während der Reaganomics ist schwierig, weil die Wirkungen auf Innovations- und Investitonsverhalten der Unternehmen nicht quantifizierbar ist.

44 Vgl. Kurz, R., Angebotsorientierte Wirtschaftspolitik in den USA: Grundlagen. Praxis und Konsequenzen, Tübingen 1993, S. 156-187.

45 Ebenda, S. 159-171.

46 Ebenda, S. 182-184. 


\subsection{Geldpolitik}

In der Einleitung zum Wirtschaftsprogramm von Ronald Reagan „A Program for Economic Recovery“ wird die Rolle der Geldpolitik folgendermaßen gesehen:

.A new commitment to a stable monetary policy." ${ }^{47}$

Die neue Verpflichtung für eine stabile Geldpolitik, die Präsident Reagan versprach, konnte er im Sinn einer angebotsorientierten Wirtschaftspolitik kaum beeinflussen.

Denn ähnlich wie in der Bundesrepublik Deutschland kann die amerikanische Zentralbank (Federal Reserve Board) ihre geldpolitischen Entscheidungen vollkommen autonom und unabhängig von der jeweiligen Regierungspolitik treffen.

Der Präsident und das Direktorium des geldpolitischen Entscheidungsorgans, das Board of Governors, werden zwar vom amerikanischen Präsidenten ernannt und sind gehalten, die allgemeine Wirtschaftspolitik der Regierung zu unterstützen, sind aber bei der Bestimmung ihres geldpolitischen Konzepts und deren Maßnahmen nicht an Regierungsanweisungen gebunden. ${ }^{48}$

In den 70er Jahren war die geldpolitische Konzeption der amerikanischen Zentralbank, die Zinsen auf einem niedrigen Niveau zu halten, damit sich die Unternehmen zur Finanzierung ihrer geplanten Investitionen günstig finanzieren konnten. ${ }^{49}$ Diese Ausrichtung der Geldpolitik führte in Verbindung mit der zweiten Ölkrise dazu, daß die Inflationsrate in den Vereinigten Staaten Ende der 70er Jahre stark anstieg.

Im Oktober 1979 entschloß der Federal Reserve Board, sein geldpolitsches Konzept grundlegend zu ändern und sich nunmehr auf die Bekämpfung der Inflation zu konzentrieren. Ein knappes Geldangebot und eine scharfe Kontrolle der Geldmengenexpansion sollten sicherstellen, daß zusätzliches Geld nur zur Finanzierung der Produktionssteigerung von Gütern und Dienstleistungen verwendet wird, und nicht zur Finanzierung des Preisanstieges..$^{50}$ Dieser Kurs einer monetaristischen Geldmengensteuerung wurde auch nach der angebotsorientierten Wende 1981 vom Federal Reserve Board weiterverfolgt. ${ }^{51}$ Eine Koordination von Geld- und Fiskalpolitik zur Stabilisierung des Konjunkturzyklusses war nunmehr tabu. .52

Um die Ausweitung der Geldmenge besser kontrollieren zu können, gab die Zentralbank jetzt jeweils für das kommende Jahr einen Zielkorridor mit einer Angabe von Un-

47 Vgl. The White House. Office of the Press Secretary: A Program for Economic Recovery, Washington D.C., February 18, 1981, S. 1

48 Vgl. Holtfrerich, C.-L.. Wirtschaft USA, München 1991, S. 92-97.

$49 \mathrm{Vgl}$. Schaal, P., Reaganomics - Konzept und Perspektiven der neuen amerikanischen Wirtschaftspolitik, List-Forum. Band 11 (1982), S. 398-399.

50 Ebenda. S. 399.

51 Vgl. Kurz, R., Angebotsorientierte Wirtschaftspolitik in den USA: Grundlagen, Praxis und Konsequenzen, Tübingen 1993. S. 67.

52 Vgl. Tobin. J., Policies for Prosperity: Essays in a Keynesian Mode, Monetarism: An Ebbing Tide?, Brighton 1987, S. 271. 
ter- und Obergrenzen der Geldmengenexpansionsrate in Prozent pro Jahr für mehrere Geldmengenaggregate vor.

Primäre Steuerungsgröße wurde die Geldmenge M1. Neben dieser Geldmenge M1 versuchte der Federal Reserve Board auch die Zuwachsraten weiterer Geldmengenaggregate (M2 und M3) innerhalb der angekündigten Zielkorridore zu halten, was jedoch nicht immer gelang. ${ }^{53}$

Ein Kennzeichen der restriktiven Geldpolitik der amerikanischen Zentralbank und die Orientierung an der Preissteigerungsrate war das Entstehen einer inversen Zinskurve in den Jahren 1979 bis 1981, bei der die kurzfristigen Zinsen über den langfristigen Zinsen lagen.

So lag der Geldmarktzins für Tagesgeld, die Federal-Funds Rate, in den Jahren 1979 bis 1981 über dem langfristigen Zinssatz für Staatsanleihen mit einer Laufzeit zwischen 10 und 30 Jahren (Long Term Rate).

Tabelle 18: Die Geldpolitik der amerikanischen Zentralbank in den Jahren 1978 - 1983

\begin{tabular}{|l|c|c|c|c|c|c|}
\hline & $\mathbf{1 9 7 8}$ & $\mathbf{1 9 7 9}$ & $\mathbf{1 9 8 0}$ & $\mathbf{1 9 8 1}$ & $\mathbf{1 9 8 2}$ & $\mathbf{1 9 8 3}$ \\
\hline $\begin{array}{l}\text { Federal Funds } \\
\text { Rate }^{1}\end{array}$ & $7,93 \%$ & $11,19 \%$ & $13,36 \%$ & $16,38 \%$ & $12,26 \%$ & $9,09 \%$ \\
\hline Long Term Rate $^{2}$ & $8,41 \%$ & $9,44 \%$ & $11,46 \%$ & $13,91 \%$ & $13,00 \%$ & $10,79 \%$ \\
\hline Inflation' & $7,6 \%$ & $11,3 \%$ & $13,5 \%$ & $10,3 \%$ & $6,2 \%$ & $3,2 \%$ \\
\hline
\end{tabular}

1: Geldmarktzins für Tagesgeld (Jahresdurchschnitt)

2: Zinssatz für festverzinsliche Staatsanleihen mit einer Laufzeit zwischen 10 und 30 Jahren (Jahresdurschnitt)

3: durchschnittliche jährliche Veränderungsrate des Konsumentenpreisindexes

Quelle: Economic Report of the President. Februar 1994. S. 340 und S. 353.

Die Tabelle zeigt, daß sich erst mit dem Rückgang der Inflation in den Jahren 1982 bis 1983 der restriktive geldpolitische Kurs der amerikanischen Zentralbank lockerte. Dennoch blieb das Jahr 1982, in dem die Steuerbeschlüsse der ersten großen Steuerreform (The Economic Recovery Tax Act) erstmals entlastend für die Unternehmen und privaten Haushalte wirksam wurden, nach wie vor durch ein hohes Zinsniveau bestimmt.

Die Supply-Sider machten später die Politiker dafür verantwortlich, daß eine derartige restriktive Geldpolitik von der Zentralbank eingeschlagen wurde. Nach ihrer Ansicht wollten die konservativen Politiker einen schnellen Sieg über die Inflation, um ihre wirtschaftspolitische Kompetenz nach dem Wahlsieg durch Ronald Reagan deutlich zu machen. ${ }^{54}$

53 Vgl. Winkler. A.. Geld. Zins und keynesianische Angebotspolitik. Berlin 1992. S. 193-234.

54 Vgl. Roberts, P. G.. Angebotsorientierte Wirtschaftspolitik, theoretische Grundlagen und politische Erfolge: Eine Bewertung der amerikanischen Erfahrungen in den achtziger Jahren. in: Zeit- 
Dies kann jedoch nur die halbe Wahrheit sein, zumal schon im Herbst 1979, ein Jahr bevor Ronald Reagan gewählt wurde, klar war, welchen geldpolitischen Kurs die amerikanische Zentralbank in nächster Zeit fahren würde

Das Ergebnis dieses ungünstigen Policy-Mix zwischen Geld- und Finanzpolitik war ein Rückgang des Wirtschaftswachstums (-2,2\%) und damit ein Anstieg der Arbeitslosenquote auf $7,1 \%$ im Jahr 1982.55

Am 1. Juli 1982 vollzog die Federal Reserve Bank einen Kurswechsel in der Geldpolitik. Für das vierte Quartal 1982 wurde auf eine Zielvorgabe für die Wachstumsrate von $\mathrm{M} 1$ verzichtet. Dies hing damit zusammen, daß trotz hoher Zinssätze die Geldmenge M1 zu Beginn des Jahres 1982 im Vorjahresvergleich stark anstieg. Für die Schwierigkeiten bei der Einhaltung bzw. Verfehlung der Zielkorridore für die Entwicklung der Geldmengenaggregate wurden Finanzinnovationen verantwortlich gemacht, die zu Verzerrungen führen und eine sinnvolle Interpretation ihrer Entwicklung unmöglich machen würden. ${ }^{56}$ Ab Juli 1982 wurde die Zinspolitik der Federal Reserve Bank verstärkt an den realwirtschaftlichen Erfordernissen ausgerichtet. Die Geldmengenorientierung rückte bei der Gestaltung der Geldpolitik in den Hintergrund. ${ }^{57}$

Schließlich wurde ab November 1985 die Zinspolitik der amerikanischen Zentralbank ganz von der Entwicklung der Geldmenge abgekoppelt. Die Antiinflationspolitik wurde wieder durch das Ziel der Wachstumsförderung ersetzt. ${ }^{58}$ Von nun an wurden die Zielvorgaben von $\mathrm{M} 1$ so formuliert, daß sie praktisch nicht verletzt werden konnten, weil in die Zielsetzung einging, daß Wachstumsraten unter- oder oberhalb des angestrebten Bereichs aus realwirtschaftlichen Gründen gerechtfertig sein könnten. ${ }^{59}$ Die strikte monetaristische Ausrichtung der Federal Reserve Bank wurde damit aufgegeben.

Entsprechend dem Programm von Ronald Reagan, welches eine neue Übereinkunft für eine stabile Geldpolitik zu Beginn seiner Amtszeit versprach, gelang es die Inflationsrate von 10,3\% im Jahr 1981 auf 1,9\% im Jahr 1986 zu senken. ${ }^{60}$ Ein beachtliches antiinflatorisches Ergebnis der unabhängigen amerikanischen Zentralbank, dennoch oder gerade deswegen wurde die Geldpolitik der Federal Reserve Bank zum Problem für die Wirtschaftspolitik Ronald Reagans. In den ersten Jahren war die Geldpolitik stark restriktiv ausgerichtet und wirkte in der rezessiven konjunkturellen Phase in den Jahren 1981 und 1982 zusätzlich konjunkturdämpfend. Hinzu kam, daß die in dieser Zeit stark anwachsenden Budgetdefizite des Bundeshaushaltes, zusammen mit dem hohem Zinsniveau, die Belastungen für den Haushalt weiter erhöhten.

Die Tabelle 19 zeigt einen Vergleich wichtiger ökonomischer Referenzgrößen der Jahre 1975 bis 1980 und 1981 bis 1986 und bestätigt, daß die wirtschaftliche Entwick-

schrift für Wirtschaftspolitik. Jg. 38 (1989), Heft 1, S. 6.

$55 \mathrm{Vgl}$. Tabelle 16.

56 Vgl. Lamping, H., Supply-Side Economics, Oldenburg 1988, S. 167.

57 Vgl. Winkler, A., Geld. Zins und keynesianische Angebotspolitik, Berlin 1992, S. 241-245.

$58 \mathrm{Vgl}$. Lamping. H., Supply-Side Economics. Oldenburg 1988, S. 168.

59 Vgl. Winkler, A.. Geld, Zins und keynesianische Angebotspolitik, Berlin 1992, S. 261.

$60 \mathrm{Vgl}$. Economic Report of the President, Februar 1994, Table B-62., S. 339. 
lung der Vereinigten Staaten in den ersten Jahren der Reagan-Regierung im Vergleich zu den Vorjahren von einem hohen Zinsniveau, stark anwachsenden Budgetdefiziten und Zinsausgaben bestimmt war. Eine konjunkturelle Wende gelang nicht, die durchschnittliche Veränderungsrate des realen Bruttoinlandsprodukts in den Jahren 1981 bis 1986 entsprach der Wachstumsrate in den Jahren 1975 bis 1980. Bemerkenswert ist der starke Rückgang der Inflationsrate, sicherlich ein Indiz für die auf Preisstabilität ausgerichtete Geldpolitik der Federal Reserve Bank nach dem zweiten Ölpreisschock.

Tabelle 19: Wirtschaftswachstum. Zinsen. Budgetentwicklung und Preisniveau in den Vereinigten Staaten im Vergleich der Jahre 1975 bis 1980 und 1981 bis 1986. in \%

\begin{tabular}{|l|c|c|}
\cline { 2 - 3 } \multicolumn{1}{c|}{} & $\mathbf{1 9 7 5 - 1 9 8 0}$ & $\mathbf{1 9 8 1 - 1 9 8 6}$ \\
\hline Bruttoinlandsprodukt $^{1}$ & $2,6 \%$ & $2,6 \%$ \\
\hline Federal Funds Rate $^{2}$ & $8,2 \%$ & $10,5 \%$ \\
\hline Long Term Rate $^{\mathbf{3}}$ & $8,7 \%$ & $11,5 \%$ \\
\hline Inflation $^{4}$ & $9,0 \%$ & $4,9 \%$ \\
\hline Budgetdefizit $^{5}$ & $2,3 \%$ & $4,2 \%$ \\
\hline Zinsausgaben $^{6}$ & $7,3 \%$ & $11,8 \%$ \\
\hline
\end{tabular}

1: durchschnittliche jährliche Veränderungsrate des Bruttoinlandsprodukts in Preisen von 1987

2: Geldmarktzins für Tagesgeld (Jahresdurchschnitt)

3: Zinssatz für festverzinsliche Staatsanleihen mit einer Laufzeit zwischen 10 und 30 Jahren (Jahresdurchschnitt)

4: durchschnittliche jährliche Veränderungsrate des Konsumentenpreisindexes

5: Budgetdefizit des Bundes in Anteilen am nominalen Bruttoinlandsprodukt

6: Zinsausgaben des Bundes in Anteilen am nominalen Bruttoinlandsprodukt

Quelle: National Income and Product Accounts of the United States, Volume 2, 1959-88. U.S. Department of Commerce, Economic Report of the President, Februar 1994, eigene Berechnungen.

Bei wachsendem Budgetdefizit und anfänglicher hoher Inflationsrate wurde die Preisniveaustabilität zur entscheidenden Referenzgröße für die Geldpolitik der amerikanischen Zentralbank. Das jeweilige geldpolitische Konzept der Federal Reserve Bank zur Erreichung dieses Zieles spielte dabei nur eine untergeordnete Rolle.

Bei der Geldpolitik zeigte sich, daß das theoretische Konzept der Supply-Side Policy das Zusammenwirken von Fiskal- und Geldpolitik wenig berücksichtigte. Dieser konzeptionelle Mangel entwickelte sich für die Wirtschaftspolitik Reagans, neben den binnenwirtschaftlichen Problemen, auch zu einem außenwirtschaftlichen Problem. 


\subsection{Die Entwicklung des Dollars und das Zwillingsdefizit}

Der Dollar-Kurs unterlag während der Amtszeit Ronald Reagans starken Schwankungen. In der DM/Dollar-Relation mußten im Jahr 1980 im Jahresdurchschnitt 1,82 DM für einen Dollar bezahlt werden. Im Jahr 1985 mußten 2,94 DM und drei Jahre später, 1988, nur noch 1,76 DM für eine Dollar aufgewendet werden.

Diese Auf- und spätere Abwertung des Dollars in den 80er Jahren traf nicht nur für die DM/Dollar-Relation, sondern genauso für das Verhältnis zu allen wichtigen Währungen der großen Industrienationen zu. Insbesondere der japanische Yen wurde in diesem Zeitraum in einer Spanne von 227 Yen für einen Dollar (1980), über 249 Yen (1982) bis zu 128 Yen im Jahr 1988 gehandelt.61

Der Anstieg und Fall des internationalen Wertes des Dollars reflektiert die grundlegenden Veränderungen in der amerikanischen Geldpolitik und im Haushaltsbudget dieser Jahre.

Die Graphik 8 zeigt die Schwankungsbreite des Dollars im Vergleich zur Deutschen Mark in den Jahren 1970 bis 1992. Im Jahr 1970 lag der Dollar im Jahresdurchschnitt noch bei 3,65 DM für einen Dollar, nach dem Zusammenbruch des Bretton-Woods Systems im Jahr 1973 mit einem Wert von 2,67 DM pro Dollar, erfolgte eine kontinuierliche Abwertung auf 1,82 DM im Jahr 1980. Danach kam es zu einem Anstieg des Dollars auf 2,94 DM im Jahr 1985, worauf wiederum Jahre der Abwertung folgten, die den Dollar im Jahr 1992 im Jahresdurchschnitt nur noch 1,56 DM Wert sein ließen.

Die Wertschwankungen des Dollars in der Phase der Reaganomics veränderten die relativen Preise für amerikanische und ausländische Güter. Die Dollarentwicklung führte dazu, daß sich Mitte der 80er Jahre ein sehr großes Handelsbilanzdefizit einstellte.

Im Jahr 1980 war die Handelsbilanz der Vereinigten Staaten mit einem Defizit von 19,7 Mrd. US-Dollar noch nahezu ausgeglichen. Drei Jahre später lag das Handelsbilanzdefizit bei 52,4 Mrd. Dollar, im Jahr 1987 erreichte das Defizit einen negativen Spitzenwert von 152,1 Mrd. Dollar, was in Anteilen am nominalen Bruttoinlandsprodukt $-3,35 \%$ bedeutet, 1980 waren es noch $-0,73 \%$ gewesen. ${ }^{62}$

Seit 1988 nimmt das Handelsbilanzdefizit ab. In Folge des hohen Defizits baute die U.S.A. eine Kapital-Schuldnerposition gegenüber dem Ausland auf. Aus einer NettoGläubiger-Position, in der sich die Vereinigten Staaten 1981 befanden, wurde ab 1985 eine Schuldnerposition. ${ }^{63}$

..The United States in these years was converted, with remarkable speed, from the world's greatest creditor nation to its greatest debtor." 64

61 Ebenda. Table B-110.. S. 394.

62 Ebenda. S. 390 und eigene Berechnungen.

$63 \mathrm{Vgl}$. Kurz. R.. Angebotsorientierte Wirtschaftspolitik in den USA: Grundlagen. Praxis und Konsequenzen, Tübingen 1993, S. 86.

64 Vgl. Galbraith. J. K.. Money, Whence it came, where it went, Boston - New York 1995. S. 308. 


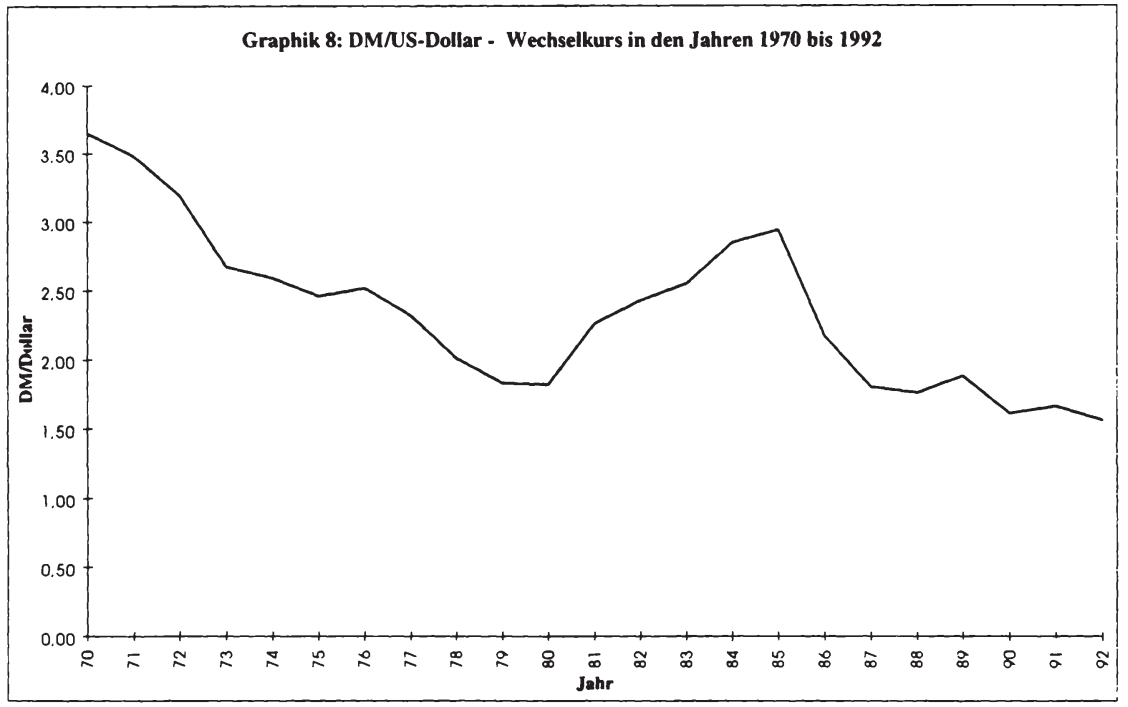

Quelle: Economic Report of the President, Februar 1994, S. 394, eigene Darstellung.

Die Steuersenkungspolitik und die hohen Staatsausgaben der Reagan-Regierung führten dazu, daß das Budget-Defizit des Bundeshaushaltes anstieg. Im Zusammenhang mit dem Handelsbilanzdefizit sprach man nunmehr von einem "Twin-Deficit“: ein Budget- und gleichzeitig ein Handelsbilanzdefizit, das die U.S.A. vorzuweisen hatte. ${ }^{65}$

Die Ursache für das Zwillingsdefizit läßt sich nicht auf einen ökonomischen Nenner bringen, es bestehen jedoch durchaus Interdependenzen. ${ }^{66}$

Der Anstieg des Dollars begann im Jahr 1980, nachdem er seit 1971 aufgrund der hohen Inflationsraten und der sich hieraus ergebenden niedrigen realen Rendite für Dollar-Anlagen gefallen war. Der Anstoß für den Anstieg kam von der restriktiven Geldpolitik der Federal Reserve Bank seit Ende 1979.

Nach der Wahl Ronald Reagans mußte die Federal Reserve Bank ihren restriktiven geldpolitischen Kurs beibehalten, weil aus ihrer Sicht aufgrund der hohen Budgetdefizite weiterhin Inflationsgefahr bestand, der mit hohen Zinsen begegnet werden mußte.

Aufgrund des Anstiegs des realen Zinsniveaus und des zurückgehenden Inflationsrisikos gewannen Dollar-Anlagen für ausländische Investoren immer mehr an Attraktivität.

65 Vgl. Krugman, P., Peddling Prosperity: economic sense and nonsense in the age of diminished expectations, New York-London 1994, S. 127.

66 Vgl. Richardson, J. D., Trade Policy, in Feldstein, M. (Hrsg.), American Economic Policy in the 1980s, Chicago 1994, S. 630. 
Der starke Anstieg des Dollars führte unzweifelhaft dazu, daß die US-Importe stark anstiegen und es bei den Exporten zu einer Stagnation kam. ${ }^{67}$ Tobin beschreibt diesen Zusammenhang folgendermaßen:

.In the 1980s, thanks to our monetary and fiscal policies, interest rates in the United States were high, even higher than interest rates in other advanced economies ... The result was a big demand for dollars relative to other currencies which bid up the price of a dollar in yen and other major currencies. The dollar became costly to foreigners; other currencies became inexpensive to Americans. The further result was that people didn't buy many American goods, while we bought a lot of foreign goods at bargain dollar prices. We developed the massive surplus of imports over exports that is still reported every month." 68

Die Graphik 9 zeigt die Entwicklung des US-Dollars im Vergleich zur Deutschen Mark in den Jahren 1981 bis 1992. Dem DM/Dollar-Kurs wird die Zinsentwicklung am Beispiel der Federal Funds Rate gegenübergestellt. In der graphischen Veranschaulichung bestätigt sich, daß mit dem Zinsanstieg der Dollarkurs in Relation zur Deutschen Mark aufgewertet wurde. Erst als die Zinsen Mitte der 80er Jahre zurückgingen verlor der Dollar gegenüber D-Mark wieder an Wert.

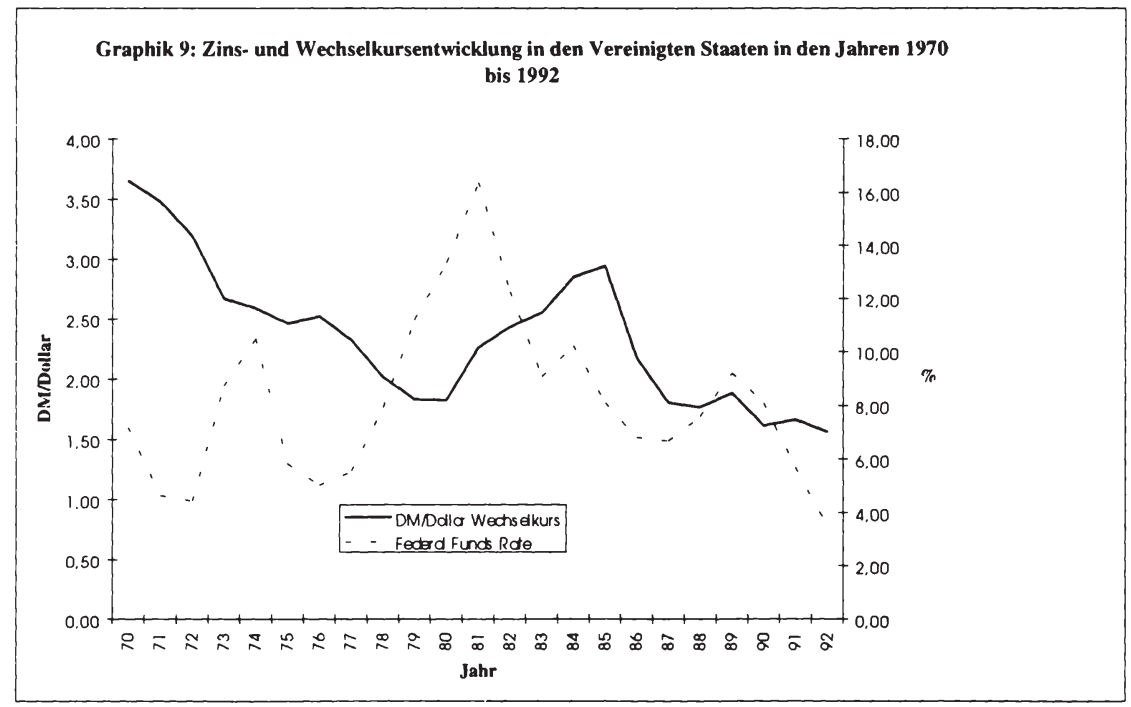

Quelle: Economic Report of the President. Februar 1994, S. 353, S. 394, eigene Darstellung.

67 Vgl. Feldstein, M.. American Policy in the 1980s: A personal view, in Feldstein, M. (Hrsg.), American Economic Policy in the 1980s, Chicago 1994, S. 65.

$68 \mathrm{Vgl}$. Tobin, J., The Monetary and Fiscal Policy Mix, in: Recent Developments in Macroeconomics, Phelps, E. S. (Hrsg.), 1991, S. 510. 
Das Handelsbilanzdefizit der Vereinigten Staaten gibt auch einen Hinweis darauf. wie sich die Sparquote der Haushalte, der Industrie und der Regierung verändert hat. Das nationale Sparaufkommen abzüglich der Investitionen entspricht den Exporten minus den Importen. Dieser grundlegende Zusammenhang gilt auch für die U.S.A..

Wenn ein Land mehr spart als es investiert hat es einen Überschuß erwirtschaftet, der exportiert werden kann.

Umgekehrt heißt dies, wenn mehr investiert als gespart wird, beanspruchen die zusätzlichen Investitionen einen Zufluß an Ressourcen vom Ausland.

Die Federal Reserve Bank of New York kommt in ihrer Studie zur Supply Side Policy zu dem Ergebnis, daß das Budgetdefizit des Bundes durch den privaten Sektor finanziert wurde. Der Betrag der Bundesschulden, der von Privaten gehalten wurde, lag in Anteilen am Bruttosozialprodukt im Jahr 1980 bei $27 \%$ und stieg bis 1990 auf $45 \% .{ }^{69}$

Mit einem Anwachsen des Budgetdefizites reduziert sich das nationale Sparaufkommen. Das Sparaufkommen des privaten Sektors und der Bundesstaaten und Gemeinden lag von 1981 bis 1990 bei 7,1\% des Bruttosozialproduktes, wurde aber durch das Budgetdefizit von 3,6\% in diesem Zeitraum auf 3,5\% reduziert. Von 1971 bis 1980 lag der vergleichbare Wert noch bei 7,0\%. ${ }^{70}$

Diese Gegenüberstellung des Sparaufkommens bestätigt die Aussage der Federal Reserve Bank of New York, daß jeder Dollar des Budgetdefizits einen verlorengegangenen Dollar des nationalen Sparens repräsentiert. ${ }^{71}$

Ein Teil des Budgetdefizits wurde durch ausländisches Kapital finanziert. Was auf der einen Seite verhinderte, daß das nationale Sparaufkommen weiter sank, aber andererseits auf lange Frist eine zusätzliche Belastung für die amerikanische Wirtschaft bedeutete. Der Schuldendienst auf das ausländische Kapital bindet zukünftiges Sparaufkommen. Der internationale Zufluß an Kapital in die Vereinigten Staaten betrug von 1981 bis 1990 im Durchschnitt 1,9\% des Bruttosozialprodukts. Dies erklärt auch, warum die U.S.A. in diesen Jahren ihre Netto-Gläubigerposition (13\% des Bruttosozialprodukts im Jahr 1980) in eine Netto-Schuldnerposition ( $-7,5 \%$ des Bruttosozialprodukts im Jahr 1990) transformierte..$^{72}$

Mit den Zinssenkungen der Federal Reserve Bank ab Mitte 1985 sank der Kurs des Dollars rapide. Ende 1987 lag er bei ca. 1,80 DM pro Dollar, zwei Jahre zuvor bei 2.94 DM. ${ }^{73}$ Dies bedeutete gegenüber der Deutschen Mark eine Abwertung von fast $40 \%$ innerhalb von zwei Jahren. ${ }^{74}$ Das Handelsbilanzdefizit der Vereinigten Staaten verbesserte sich nur allmählich. Erst im Jahr 1988 ging es erstmals wieder zurück, blieb aber

69 Vgl. Akhtar, M.A.. Harris, E.S., The Supply-Side Consequence of U.S. Fiscal Policy in the 1980s. in: Federal Reserve Bank of New York, Quarterly Review, Vol. 17. Nr.1, 1992. S. 3.

70 Ebenda, Table 3, S. 4.

71 Ebenda. S. 3.

72 Ebenda. S. 5.

73 Vgl. Graphik 9.

74 Vgl. Economic Report of the President, Februar 1994, S. 394. 
nach wie vor auf einem hohen Niveau. Die Graphik 10 zeigt die Entwicklung des amerikanischen Handelsbilanzdefizits in den Jahren 1982 bis 1992.

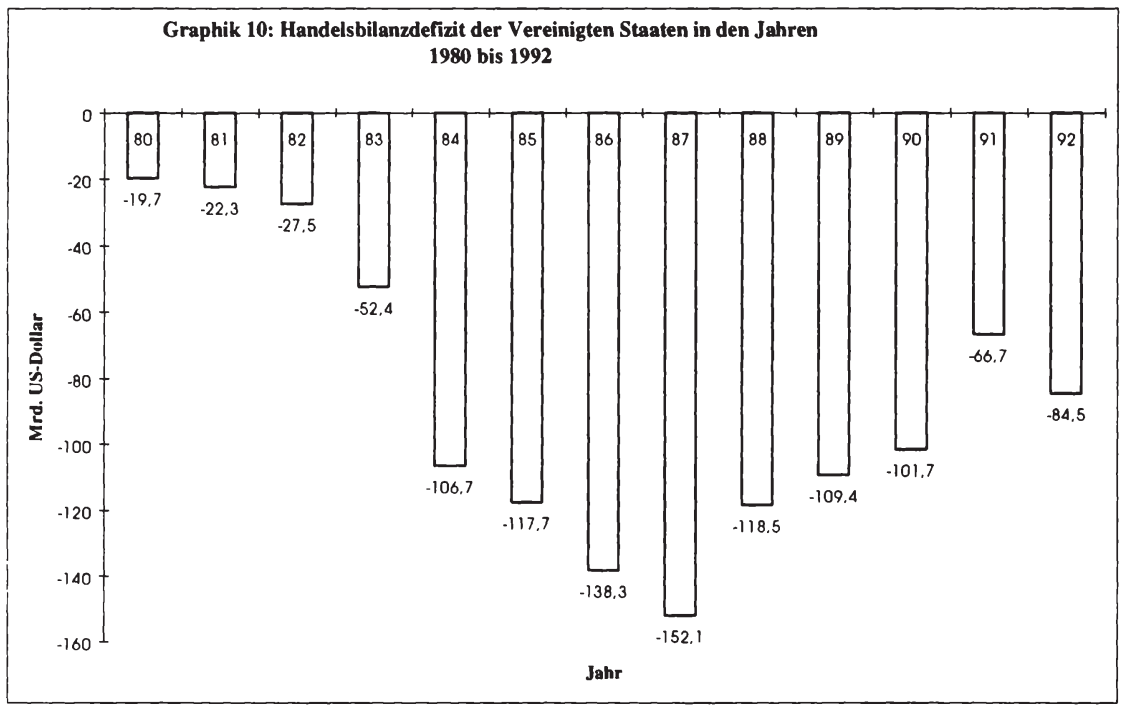

Quelle: Economic Report of the President, Februar 1994, S. 390, eigene Darstellung.

Neben den Zinssenkungen der amerikanischen Zentralbank kam hinzu, daß die Politik der Reagan-Regierung ihre internationale Grundhaltung gegenüber dem hohen AuBenwert des Dollars änderte. Bis zum September 1985 wurde offiziell die Position vertreten, daß der starke Dollarkurs die hohe Wertschätzung des Auslandes in die amerikanische Wirtschaft signalisiere. Als jedoch das Außenhandelsdefizit immer größer wurde, gab man diese Einschätzung auf. Am 22. September 1985 erklärten die G-5 Länder (Deutschland, Japan, Frankreich, England und U.S.A.) bei ihrem Treffen im Plaza Hotel in New York im sogenannten „Plaza Agreement“, daß der Dollarkurs sinken müsse. ${ }^{75}$ Diese gemeinsame Feststellung der wichtigsten westlichen Industrienationen stellte einen Wendepunkt für die Dollarentwicklung dar. ${ }^{76}$ In Folge dieser internationalen Übereinkunft für die zukünftige Dollarkursentwicklung kam es zu koordinierten Devisenmarkttransaktionen der G-5 Länder. ${ }^{77}$

75 Vgl. Feldstein, M., American Policy in the 1980s: A personal view, in Feldstein, M. (Hrsg.), American Economic Policy in the 1980s, Chicago 1994, S. 72.

76 Vgl. Frankel, J. A., Exchange Rate Policy, in Feldstein, M. (Hrsg.), American Economic Policy in the 1980s, Chicago 1994, S. 302.

77 Vgl. Kurz, R., Angebotsorientierte Wirtschaftspolitik in den USA: Grundlagen, Praxis und Konsequenzen, Tübingen 1993. S. 87. 
Die angebotsorientierte Wirtschaftspolitik Ronald Reagans führte im Zusammenhang mit der restriktiven Geldpolitik der amerikanischen Zentalbank dazu, daß ein Zwillingsdefizit in den Vereinigten Staaten in den 80er Jahren entstehen konnte. Die Steuersenkungen und das hohe Zinsniveau lenkten die interrnationalen Kapitalströme in die U.S.A.. Die Finanzierung des Budgetdefizits, das aufgrund der hohen Staatsausgaben entstand, band inländisches Kapital, das damit nicht mehr für Investitionen verwendet werden konnte und wandelte die Gläubigerposition der U.S.A. in eine Schuldenerposition gegenüber dem Ausland. Die Wirtschaftspolitik Reagans hat die internationale Wettbewerbsfähigkeit der U.S.A. sicherlich nicht verbessert, sie hat wohl eher dazu geführt, da $B$ ihr Anliegen der Investitionsstärkung über die Budgetdefizite konterkariert wurde und zusätzlich ein Zwillingsdefizit entstehen konnte.

Die Graphik 11 zeigt, welche Größenordnungen das Zwillingsdefizit der U.S.A. in den 80er Jahren erreichte. In der graphischen Veranschaulichung wird das Zwillingsdefizit (Budgetdefizit und Handelsbilanzdefizit) in Relation zum nominalen Bruttosozialprodukt gesetzt.

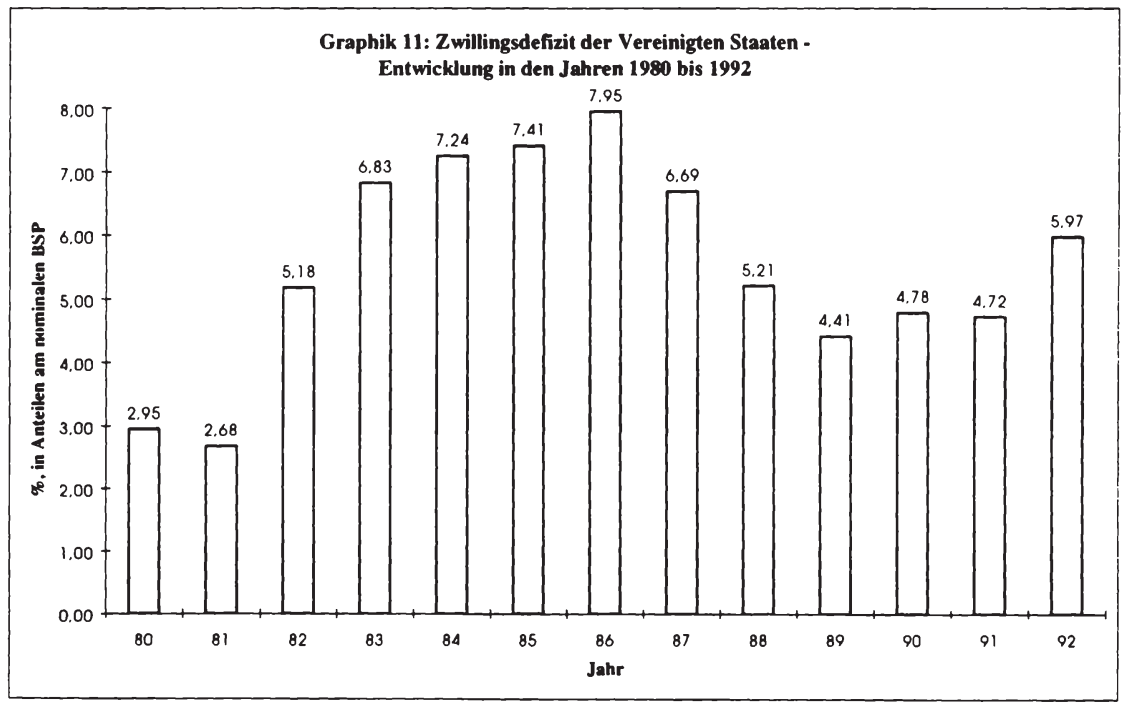

Quelle: Economic Report of the President, Februar 1994, eigene Berechnung und Darstellung. 


\subsection{Die Reagan-Projektion und was daraus wurde}

Die Tabelle 20 greift die wirtschaftliche Projektion auf, die Ronald Reagan in seinem Wirtschaftsprogramm „A Program for Economic Recovery“ für Staatseinnahmen, Staatsausgaben, Bruttosozialprodukt und Budgetsaldo für den Zeitraum von 1981 bis 1986 abgegeben hat und vergleicht sie mit der tatsächlichen Entwicklung in den Vereinigten Staaten in dieser Zeit. ${ }^{78}$

Tabelle 20: Die tatsächliche wirtschaftliche Entwicklung in den Vereinigten Staaten im Vergleich mit der Reagan - Projektion, in Mrd. US-Dollar und in \%

\begin{tabular}{|c|c|c|c|c|c|c|c|}
\hline & & 1981 & 1982 & 1983 & 1984 & 1985 & 1986 \\
\hline \multirow{2}{*}{$\begin{array}{l}\text { Staats-' } \\
\text { einnahmen }\end{array}$} & tatsächl. & 639,0 & 635,4 & 660,0 & 725,8 & 788,6 & 827,2 \\
\hline & Reagan & 600,2 & 650,5 & 710,2 & 772,1 & 850,9 & 942,1 \\
\hline \multirow{2}{*}{$\begin{array}{l}\text { Einnahmen } \\
\text { Quote }^{2}\end{array}$} & tatsächl. & $21,1 \%$ & $20,2 \%$ & $19,4 \%$ & $19,2 \%$ & $19,5 \%$ & $19,4 \%$ \\
\hline & Reagan & $21,1 \%$ & $20,4 \%$ & $19,7 \%$ & $19,3 \%$ & $19,3 \%$ & $19,6 \%$ \\
\hline \multirow{2}{*}{$\begin{array}{l}\text { Staats- } \\
\text { ausgaben" }\end{array}$} & tatsächl. & 697,8 & 770,9 & 840,0 & 892,7 & 969,9 & 1028,2 \\
\hline & Reagan & 654,7 & 695,5 & 733,1 & 771,6 & 844 & 912,1 \\
\hline \multirow{2}{*}{$\begin{array}{l}\text { Ausgaben } \\
\text { Quote }\end{array}$} & tatsächl. & $23,0 \%$ & $24,5 \%$ & $24,7 \%$ & $23,6 \%$ & $24,0 \%$ & $24,1 \%$ \\
\hline & Reagan & $23,0 \%$ & $21,8 \%$ & $20,4 \%$ & $19,3 \%$ & $19,2 \%$ & $19,0 \%$ \\
\hline \multirow{2}{*}{$\begin{array}{l}\text { Brutto-" } \\
\text { sozialprod. }\end{array}$} & tatsächl. & 3030,6 & 3149,6 & 3405,0 & 3777,2 & 4038,7 & 4268,6 \\
\hline & Reagan & 2844,5 & 3188,7 & 3605,1 & 4000,5 & 4408,8 & 4806,6 \\
\hline \multirow{2}{*}{$\begin{array}{l}\text { Budget- } \\
\text { saldo }\end{array}$} & tatsächl. & $-58,8$ & $-135,5$ & $-180,1$ & $-166,9$ & $-181,4$ & $-201,0$ \\
\hline & Reagan & $-54,4$ & $-45,0$ & $-22,9$ & $+0,5$ & $+6,9$ & $+29,9$ \\
\hline
\end{tabular}

1: Einnahmen des Bundes in Mrd. US-Dollar

2: Einnahmen des Bundes in Anteilen am nominalen Bruttosozialprodukt, in \%

3: Ausgaben des Bundes im Mrd. US-Dollar

4: Ausgaben des Bundes in Anteilen am nominalen Bruttosozialprodukt, in \%

5: nominales Bruttosozialprodukt in Mrd. US-Dollar

6: Budgetdefizit des Bundeshaushaltes in Mrd. US-Dollar

Quelle: The White House, Office of the Press Secretary: A Program for Economic Recovery, Washington D.C., Feb. 1981, S. 2 und S.8, National Income and Product Accounts of the United States, Volume 2, 1959-88, Economic Report of the President, Februar 1994, U.S. Department of Commerce, und eigene Berechnungen.

$78 \mathrm{Vgl}$. The White House, Office of the Press Secretary: A Program for Economic Recovery, Washington D.C., February 18, 1981 und Tabellen 12 bis 15 der vorliegenden Ausarbeitung. 
Der Vergleich der tatsächlichen wirtschaftlichen Entwicklung in den Vereinigten Staaten belegt, daß die Prognose des Reagan-Wirtschaftsprogramms für die Jahre 1981 bis 1986 nicht eingetreten ist.

Die veranschlagten Werte wurden nicht nur knapp, sondern weit verfehlt.

Bei einer Beurteilung der angebotsorientierten Wirtschaftspolitik ausschließlich an ihren tatsächlichen wirtschaftlichen Ergebnissen im Vergleich zur Reagan-Projektion müßte man feststellen, daß die angebotsorientierte Wirtschaftspolitik von Ronald Reagan versagt hat.

Die Staatseinnahmen sind in Anteilen am Bruttosozialprodukt von $21,1 \%$ (1981) auf 19,4 \% (1986) zurückgegangen. Reagan hatte für diese Größe ein Ziel von 19,6 \% vorgegeben. Eigentlich müßte man sagen, die Prognose ist eingetreten, aber weit gefehlt. Die Einnahmenquote bezieht sich auf das nominale Bruttosozialprodukt. Dieses sollte im Jahr 1986 eine Höhe von 4806,6 Mrd. Dollar erreichen, tatsächlich belief sich das Bruttosozialprodukt in diesem Jahr auf 4268,6 Mrd. Dollar; eine Lücke von mehr als 500 Mrd. Dollar im Vergleich zur Prognose. Das erhoffte Wirtschaftswachstum aufgrund der Steuersenkungen blieb aus. Aus diesem Grund blieben auch die Steuereinnahmen mit 827,2 Mrd. Dollar im Jahr 1986 mehr als 100 Mrd. Dollar unter dem Projektions-Wert von 942 Mrd. Dollar.

Ganz anders entwickelten sich die Staatsausgaben. Diese sollten, so die Vorstellung von Reagan, von 23,0 \% auf 19,0 \% in Anteilen am Bruttosozialprodukt sinken. Tatsächlich erreichte die Staatsquote im Jahr 1986 einen Wert von $24,1 \%$. Eigentlich sollten die Staatseinnahmen schneller wachsen als die Staatsausgaben, aber das Gegenteil trat ein. Die Staatsausgaben nahmen stärker zu, das Wirtschaftswachstum war schwächer als geplant und damit erhöhte sich der Staatsausgabenanteil am Bruttosozialprodukt. Im Jahr 1986 lagen die Staatsausgaben mit 1028,2 Mrd. Dollar um 116,1 Mrd. Dollar über dem geplanten Wert von 912,1 Mrd. Dollar.

In den Jahren 1981 bis 1986 wurde die Budget-Lücke zwischen Staatseinnahmen und Staatsausgaben immer größer. Das Budgetdefizit erreichte im Jahr 1986 eine Rekordhöhe von 201 Mrd. Dollar. Nach Reagan sollten sich schon 1984 ein Budgetüberschuß einstellen und im Jahr 1986 die Staatseinnahmen um 29,9 Mrd. Dollar über den Staatsausgaben liegen. Reagan hat sich 1981 um 229,9 Mrd. Dollar vertan.

Auf kurze Frist war die Wirtschaftspolitik Ronald Reagans ein Mißerfolg:

- geringes Wirtschaftswachstum,

- steigende Staatsausgaben,

- sinkende Steuereinnahmen,

- hohes Budgetdefizit.

Das Wirtschaftsprogramm Ronald Reagans aus dem Jahr 1981 suggerierte mit den Projektionswerten, daß hinter dem Programm die Annahmen der Laffer-Kurve standen. Das Wirtschaftsprogramm verfehlte seine erhoffte Wirkung auf Staatseinnahmen und Wirtschaftswachstum, war damit das Laffer-Konzept schon in den Jahren 1981 bis 1986 gescheitert? 


\subsection{Die langfristigen ökonomischen Auswirkungen der Reaganomics}

Die kuzfristige Analyse der Reaganomics belegte anhand der wirtschaftlichen Daten der Jahre 1981 bis 1986, daß, gemessen an der Reagan-Projektion, die Wirtschaftspolitik ihre geplanten Ziele verfehlte. Es wäre zu einfach, sein Urteil über die Wirtschaftspolitik Reagans und die Ergebnisse der Supply-Side Policy nur auf diesen Zeitraum zu gründen. Die Reaganomics wurde weitgehend bis zum Jahr 1992 fortgesetzt. Eine Dekade lang bestimmte sie die wirtschaftliche Entwicklung der U.S.A.. Geht man davon aus, daß mit dem Jahr 1982 die wirtschaftliche Beeinflussung der Vereinigten Staaten durch die angebotsorientierte Wirtschaftspolitik begann, repräsentiert der Zeitraum von zehn Jahren (1982 bis 1992) einen angemessenen Zeitraum für eine langfristige Analyse der Reaganomics. Obwohl Reagan bereits 1981 Präsident der Vereinigten Staaten war, macht es durchaus Sinn, dieses Jahr bei der Untersuchung der langfristigen Auswirkungen der Supply-Side Policy auszulassen. Das Jahr 1981 stand noch unter den Nachwirkungen der 2. Ölpreiskrise, und zudem wurden die steuerlichen Entlastungen durch die Tax Reform Act zum Teil erst 1982 voll wirksam. ${ }^{79}$

Die Supply-Sider sahen in ihren Ideen immer eine langfristige Lösung für die wirtschaftlichen Probleme der Vereinigten Staaten.

.... new ideas to get a solid start on the solution of our long-term problems. “ ${ }^{40}$

Ein Vergleich der Jahre 1960 bis 1969, 1970 bis 1981, 1982 bis 1992 und 1960 bis 1992 soll zeigen, welche wirtschaftlichen Ergebnisse die Reaganomics den Vereinigten Staaten gebracht hat.

\subsubsection{Wirtschaftswachstum}

Akhtar und Harris kamen in ihrer Analyse der langfristigen Auswirkungen der Reaganomics zu dem Ergebnis, daß keine grundlegende Verbesserung der wirtschaftlichen Entwicklung in den Vereinigten Staaten in den achtziger Jahren zu beobachten war. ${ }^{81}$

Dieses Ergebnis wird durch die Graphik 12, die einen typischen zyklischen Wirtschaftsverlauf zeigt, und mit der Tabelle 21 bestätigt.

79 Vgl. Roberts, P. G.. Angebotsorientierte Wirtschaftspolitik, theoretische Grundlagen und politische Erfolge: Eine Bewertung der amerikanischen Erfahrungen in den achtziger Jahren, in: Zeitschrift für Wirtschaftspolitik, Jg. 38 (1989), Heft 1, S. 6.

80 Vgl. Eckstein. O., A Time for Supply Economics, in: Fink, R. H. (Hrsg.), Supply-Side Economics: A Critical Appraisal, Maryland 1982, S. 243.

81 Vgl. Akhtar, M.A.. Harris, E.S., The Supply-Side Consequence of U.S. Fiscal Policy in the 1980s, in: Federal Reserve Bank of New York, Quarterly Review, Vol. 17, Nr.1, 1992, S. 2. 


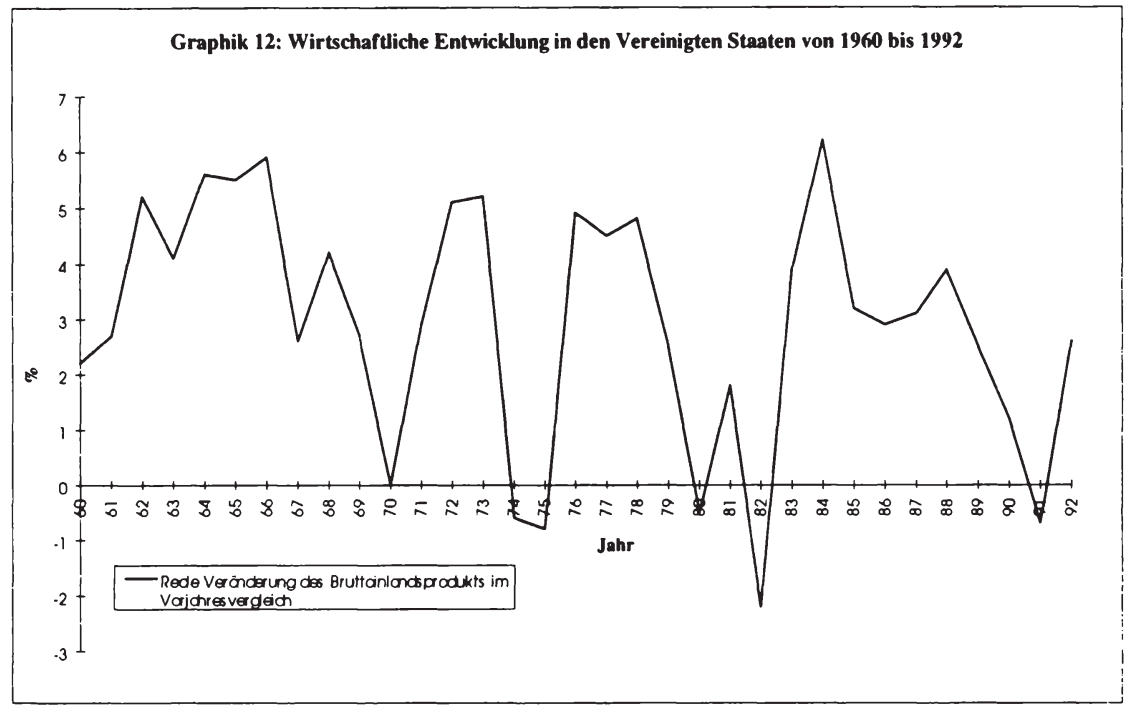

Quelle: Economic Report of the President, Februar 1994, Table B-2., S. 271, eigene Dartsellung.

Die durchschnittliche reale Wachstumsrate des Bruttoinlandsprodukts war von 1982 bis 1992 mit 2,4\% die niedrigste im Vergleich zu den übrigen Perioden. Die konjunkturelle Entwicklung in den 80er Jahren war am Anfang von den Auswirkungen durch die zweite Ölpreiskrise bestimmt. Der Tiefpunkt der Rezession war im Jahr 1982 mit einem Rückgang des realen Bruttoinlandsprodukts von $-2,2 \%$ erreicht. Danach folgte eine wirtschaftliche Aufschwungphase, die im Jahr 1984 ihren Höhepunkt mit einem Zuwachs des realen Bruttoinlandsprodukts von 6,2\% erreichte. Dieser Aufschwung ist zum Teil auch dadurch zu erklären, daß es hier zu einem Basiseffekt gekommen ist. Aufgrund der Rezession, mit dem Tiefpunkt im Jahr 1982, kehrte die Konjunktur in den Jahren 1983 bis 1984 auf ihr normales Niveau zurück. Anteil an dem Aufschwung in den Jahren 1983 bis 1984 hatten sicherlich auch die massiven Steuerentlastungen, die aufgrund der Tax Reform Act den privaten Haushalten und Unternehmen zusätzliches Einkommen verschafften. Kurzfristig erhöhte sich, getragen vom privaten Wohnungsbau und Konsum, in diesen Jahren die gesamtwirtschaftliche Nachfrage. ${ }^{82}$ In den folgenden Jahren wurden vornehmlich nur noch Wachstumsraten zwischen zwei und vier Prozent für das reale Bruttoinlandsprodukt erreicht. Im Jahr 1991 schrumpfte das reale Bruttoinlandsprodukt sogar wieder real um $-0,7 \%$.

$82 \mathrm{Vgl}$. Kurz, R., Angebotsorientierte Wirtschaftspolitik in den USA: Grundlagen. Praxis und Konsequenzen, Tübingen 1993, S. 56. 
Tabelle 21: Das durchschnittliche reale Wirtschaftswachstum in den Vereinigten Staaten, 1960 bis 1969, 1970 - 1981, 1960 - 1992 und in der Zeit der Reaganomics 1982 bis 1992, in \%

\begin{tabular}{|l|c|c|c|c|}
\cline { 2 - 5 } \multicolumn{1}{c|}{} & $1960-1969$ & $1970-1981$ & $\mathbf{1 9 8 2}-\mathbf{1 9 9 2}$ & $1960-1992$ \\
\hline Bruttoinlandsprodukt $^{1}$ & $4,1 \%$ & $2,5 \%$ & $\mathbf{2 , 4} \%$ & $2,9 \%$ \\
\hline
\end{tabular}

1: durchschnittliche jährliche Veränderungsrate des Bruttoinlandsprodukts in Preisen von 1987

Quelle: National Income and Product Accounts of the United States, Volume 2, 1959-88, U.S. Department of Commerce und Survey of Current Business, U.S. Department of Commerce, July 1992, Volume 72. Number 7 und Survey of Current Business, U.S. Department of Commerce, March 1993, Volume 73, Number 3 und eigene Berechnungen.

Das Wirtschaftswachstum in den Jahren der Reaganomics war wie in den Dekaden zuvor von starken zyklischen Schwankungen bestimmt. Ob die kurze Aufschwungphase Mitte der 80er Jahre nun tatsächlich aus der Verbesserung der Angebotsbedingungen resultierte bleibt fraglich, die Analyse der Entwicklung der Spartätigkeit und des Konsums ließe auch einen anderen Schluß zu.

Eigentlich sollten die Steuersenkungen dazu führen, daß die produktive Basis der Wirtschaft der U.S.A. vergrößert wurde. Aber auch für diese Größe gilt, daß die Produktivitätsentwicklung in den Jahren 1982 bis 1992 mit einem Zuwachs von 1,3\% auf dem durchschnittlichen Niveau der Vorperiode lag. Die Tabelle 22 und die Graphik 13 zeigen, daß es der Reaganomics nicht gelungen ist, den Rückgang der Wachstumsraten bei der Produktivität, der seit Anfang der 70er Jahre zu beobachten war, aufzuhalten. Nach Auffassung des Economic Report of the President des Jahres 1994 ist für die amerikanische Wirtschaft nichts wichtiger als das Wachstum der Produktivität, demnach hat die Reaganomics die U.S.A. wirtschaftlich nur langsam voran gebracht. ${ }^{83}$

Tabelle 22: Produktivitätsentwicklung in den Vereinigten Staaten, 1960 bis 1969, 1970 - 1981, 1960 1992 und in der Zeit der Reaganomics 1982 bis 1992, in \%

\begin{tabular}{|l|c|c|c|c|}
\cline { 2 - 5 } \multicolumn{1}{c|}{} & $1960-1969$ & $1970-1981$ & $\mathbf{1 9 8 2}-\mathbf{1 9 9 2}$ & $1960-1992$ \\
\hline Produktivität $^{\prime}$ & $2,9 \%$ & $1,3 \%$ & $\mathbf{1 , 3} \%$ & $1,8 \%$ \\
\hline
\end{tabular}

1: durchschnittliche jährliche Veränderungsrate der Produktion pro Arbeitsstunde (Output per hour of all persons - Business sector)

Quelle: Economic Report of the President, Februar 1994, Table B-48., S. 323 und eigene Berechnungen.

$83 \mathrm{Vgl}$. Economic Report of the President, Februar 1994, S. 25. 


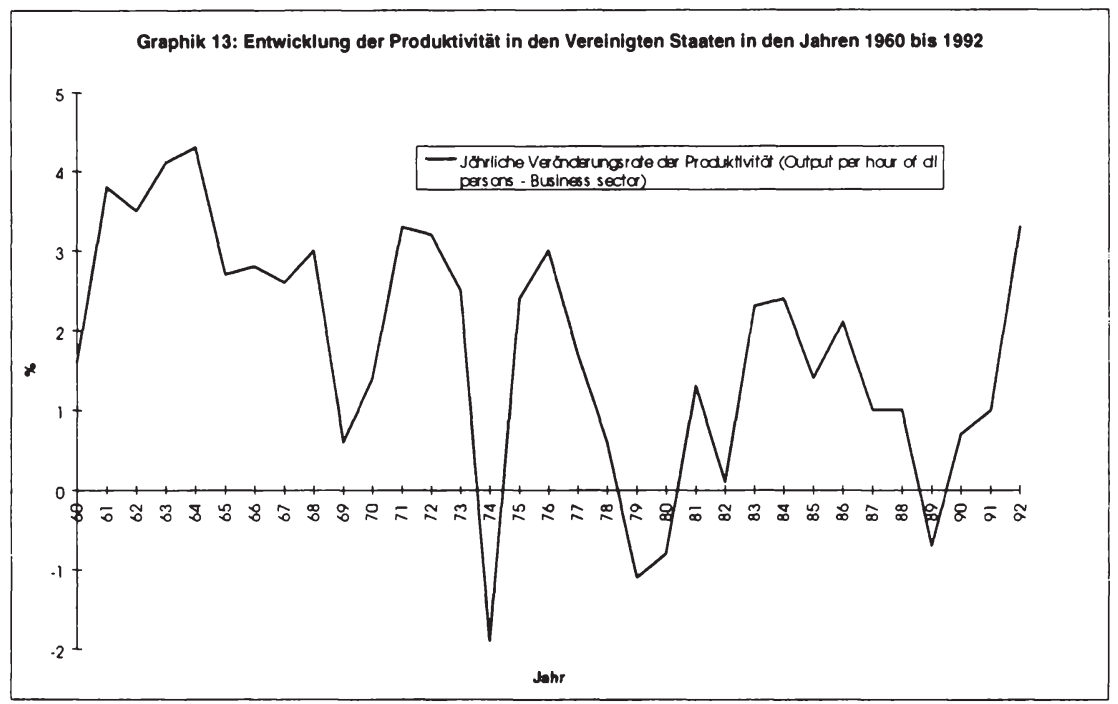

Quelle: Economic Report of the President, Februar 1994, Table B-48., S. 323.

Am 20. Januar 1981, dem Tag seiner Amtseinführung, erklärte Ronald Reagan:

..In the days ahead I will propose removing the roadblocks that have slowed our economy and reduced productivity. Steps will be taken aimed at restoring the balance between the various levels of government. Progress may be slow - measured in inches and feet, not miles - but we will progress. Is it time to reawaken the industrial giant, to get go government back within its means, and to lighten our punitive tax burdens. And these will be our first priorities, and on these principles. there will be no compromise." 84

Ronald Reagan ist es nicht gelungen die Barrieren aus dem Weg zu räumen, die das Wachstum der Wirtschaft verlangsamt haben. Die Reaganomics in den 80er Jahren hat keine besseren Ergebnisse für das Wachstum der amerikanischen Wirtschaft als die nachfrageorientierte Wirtschaftspolitik der 70er Jahre erbracht. Dies gilt auch für die Entwicklung der Investitionen und des Sparaufkommens.

84 Vgl. Reagan, R.. First Inaugural Address, 20. Januar 1981, in: http://www.columbia.edu/acis/bartleby/inaugural/pres61.html, vom 12.09.1996. 


\subsubsection{Investitionen und Sparen}

Die Supply-Sider erklärten immer wieder, daß der Zweck der Steuersenkungen u.a. eine Erhöhung der Investitionen sei. Kemp formulierte dies 1982 so:

,.... the purpose of cutting tax rates is not to create a deficit but to increase investment, employment, and the prospects for a balanced budget." ${ }^{\text { }} 5$

Diese Erklärung ist um so interessanter, weil im Präsidentschaftswahlkampf 1996 Jack Kemp im Fall eines Wahlsieges des republikanischen Präsidentschaftsbewerbers Dole das Amt des Vize-Präsidenten der Vereinigten Staaten erhalten würde. Das Wirtschaftsprogramm der Republikaner für den Wahlkampf 1996, das maßgeblich auf Kemp zurückgeht, sieht bei einem Wahlsieg eine Senkung des marginalen Steuersatzes der Einkommensteuer von $15 \%$ vor. ${ }^{86}$

Neben einer Stärkung der Investitionen war auch die Entwicklung der Sparquote für die Reaganomics wichtig. Die Voraussetzungen für mehr Wachstum waren nach ihrer Auffassung Konsumverzicht und eine Erhöhung der Sparquote. ${ }^{87}$

Unabhängig davon, welche Größe man für die Beschreibung der Investitionsentwicklung heranzieht, wurde im Zeitraum von 1982 bis 1992 weniger investiert als in den Jahren von 1970 bis 1981 (Tabelle 23).

Die Economic Recovery Tax Act aus dem Jahre 1981 sollte eigentlich den steuerlichen Anreiz für zusätzliche Investitionen auslösen. Im Sinn der Angebotstheorie wäre eine Erhöhung der Investitionsquote als Kapazitätsausbau zu interpretieren. Am geeignetsten erscheint hier die Verwendung der realen Netto-Investitionen außerhalb des Wohnungsbaus in Anteilen am realen Bruttoinlandsprodukt zu sein. Die reale Nettoinvestitionsquote beschreibt, wie sich die Anlageinvestitionen, die zur Erhöhung des Kapitalstocks dienen, in der Vergangenheit entwickelt haben. ${ }^{88}$

Für die U.S.A. erreichte die reale Nettoinvestitionsquote mit $2,4 \%$ in der Zeit von 1982 bis 1992 ihren Tiefpunkt (Tabelle 23 und Graphik 14). Inwieweit die Steuererleichterungen der Reaganomics die Investitionen in den Vereinigten Staaten beeinflußt haben, ist schwer zu beantworten. Akhtar und Harris verweisen darauf, daß das Investitionsverhalten wohl stärker durch die Zinsentwicklung, Inflation und die allgemeine wirtschaftliche Entwicklung beeinflußt wird als durch Steuersenkungen. ${ }^{89}$

Festzuhalten bleibt, daß die Steuersenkungen der Reaganomics ihr Ziel, die Investitionen zu erhöhen, gemessen an den absoluten Größenordungen für einzelne Investitionsindikatoren, verfehlt haben.

85 Vgl. Kemp, J., Supply-Side Economics: An American Renaissance, in: Supply-Side Economics in the 1980s, Federal Reserve Bank of Atlanta 1982, S. 28.

86 Vgl. Los Angeles Times, vom 13. August 1996, S. A17.

87 Vgl. Müller-Oestreich, K., Der Einfluß der Finanzpolitik auf die Investitionsentwicklung in den USA unter Ronald Reagan, Berlin 1992, S. 33-34.

88 Ebenda, S. 23-28.

$89 \mathrm{Vgl}$. Akhtar, M.A., Harris, E.S., The Supply-Side Consequence of U.S. Fiscal Policy in the 1980s, in: Federal Reserve Bank of New York, Quarterly Review, Vol. 17, Nr.1, 1992, S. 14. 
Tabelle 23: Investitionsentwicklung und Sparquote in den Vereinigten Staaten. 1960 bis 1969. 1970 1981, 1960 - 1992 und in der Zeit der Reaganomics 1982 bis 1992, in \%

\begin{tabular}{|l|c|c|c|c|}
\cline { 2 - 5 } \multicolumn{1}{c|}{} & $1960-1969$ & $1970-1981$ & $\mathbf{1 9 8 2}-\mathbf{1 9 9 2}$ & $1960-1992$ \\
\hline $\begin{array}{l}\text { Nominale Brutto- } \\
\text { Investitionen in Anteilen } \\
\text { am BIP }\end{array}$ & $15,8 \%$ & $17,2 \%$ & $\mathbf{1 5 , 9} \%$ & $16,3 \%$ \\
\hline $\begin{array}{l}\text { Nominale Brutto- } \\
\text { Investitionen außerhalb } \\
\text { des Wohnungsbaus in An- } \\
\text { teilen am BIP }\end{array}$ & $10,0 \%$ & $11,5 \%$ & $\mathbf{1 1 , 2} \%$ & $11,0 \%$ \\
\hline $\begin{array}{l}\text { Reale Brutto- } \\
\text { Investitionen in Anteilen } \\
\text { am BIP }\end{array}$ & $15,6 \%$ & $16,4 \%$ & $\mathbf{1 5 , 9 \%}$ & $16,0 \%$ \\
\hline $\begin{array}{l}\text { Reale Netto-Investitionen } \\
\text { außerhalb des Woh- } \\
\text { nungsbaus in Anteilen am } \\
\text { BIP4 }\end{array}$ & $3,2 \%$ & $3,3 \%$ & $\mathbf{2 , 4 \%}$ & $3,0 \%$ \\
\hline $\begin{array}{l}\text { Nationales Sparen } \\
\text { Sparquote }\end{array}$ & $16,6 \%$ & $17,1 \%$ & $\mathbf{1 4 , 2} \%$ & $16,0 \%$ \\
\hline
\end{tabular}

1: durchschnittlicher jährlicher prozentualer Anteil der nominalen Brutto-Investitionen in Anteilen am nominalen Bruttoinlandsprodukt

2: durchschnittlicher jährlicher prozentualer Anteil der nominalen Brutto-Investitionen außerhalb des Wohnungsbaus in Anteilen am nominalen Bruttoinlandsprodukt

3: durchschnittlicher jährlicher prozentualer Anteil der realen Brutto-Investitionen in Anteilen am realen Bruttoinlandsprodukt in Preisen von 1987

4: durchschnittlicher jährlicher prozentualer Anteil der realen Netto-Investitionen außerhalb des Wohnungsbaus in Anteilen am realen Bruttoinlandsprodukt in Preisen von 1987

5: Nationales Sparaufkommen in prozentualen Anteilen am nominalen Bruttoinlandsprodukt

6: Privates Sparaufkommen in prozentualen Anteilen am verfügbaren Einkommen

Quelle: National Income and Product Accounts of the United States, Volume 2, 1959-88, Economic Report of the President, Februar 1994, eigene Berechnung.

Das Ziel einer Erhöhung der Sparquote wurde ebenfalls nicht erreicht. Sowohl das nationale Sparaufkommen als auch das private Sparaufkommen erreichten im Zeitraum von 1982 bis 1992 ihren jeweiligen Tiefpunkt (Tabelle 23). Das private Sparaufkommen in Anteilen am verfügbaren Einkommen sank von 7,9\% auf 5,7 \% (Tabelle 23 und 
Graphik 14). Blinder hat daraus gefolgert, da $B$ der Zusammenhang zwischen ökonomischen Anreizen und der Entwicklung der Sparquote in den Vereinigten Staaten nicht besonders stark sein muß, weil trotz hoher Zinssätze und Senkung der Grenzsteuersätze das Sparaufkommen in den Vereinigten Staaten in den 80er Jahren gesunken ist. ${ }^{90}$

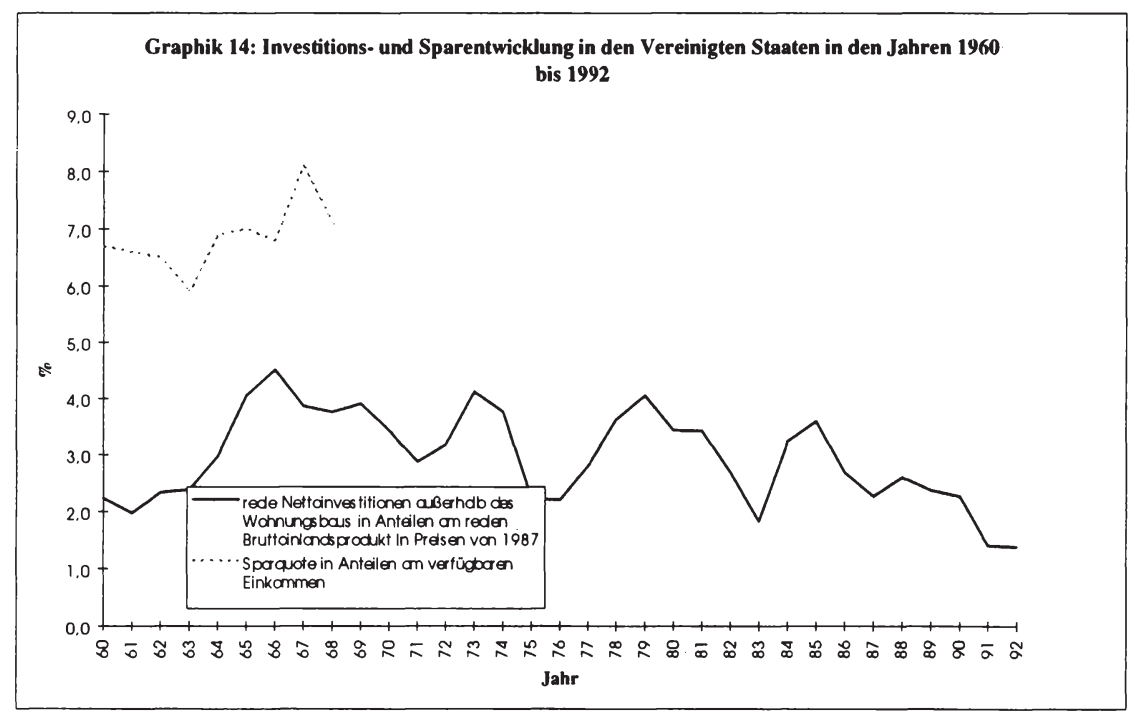

Quelle: National Income and Product Accounts of the United States, Volume 2, 1959-88, Economic Report of the President, Februar 1994, eigene Darstellung.

Ganz anders verlief die Entwicklung beim Konsum. Dieser erhöhte sich in den Jahren der Reaganomics auf einen Anteil von $66,9 \%$ am realen Bruttoinlandsprodukt (Tabelle 24). Im Sinne der Supply-Sider stellte diese Entwicklung des privaten Verbrauchs ein Paradoxon dar. Die Investitionen gingen zurück, und der Anstieg des Konsums hat im Gegenzug zur Stärkung der effektiven wirtschaftlichen Gesamtnachfrage beigetragen. Eine konjunkturstützende Komponente der Nachfrageseite, der Konsum, hat die negative Entwicklung bei den Investitionen auf der Angebotsseite in den U.S.A. in den 80er Jahren zum Teil kompensiert

Ausgehend von dieser Aussage von Akhtar und Harris hat die Supply-Side Policy keine Gültigkeit. Die angebotsorientierte Wirtschaftspolitik sollte aufgrund der niedri-

90 Vgl. Blinder, A. S., Comments on „Why is U.S. National Saving So Low ?“, Brookings Papers on Economic Activity, 2, Summers, L. und Carrol, C. (Hrsg.), 1987, S. 638. 
gen Grenzsteuersätze eigentlich dazu führen, daß mehr gearbeitet und weniger Freizeit gemacht wird. ${ }^{91}$

Tabelle 24: Entwicklung des Konsums in den Vereinigten Staaten. 1960 bis 1969. 1970 - 1981. 1960 1992 und in der Zeit der Reaganomics 1982 bis 1992, in \%

\begin{tabular}{|l|c|c|c|c|}
\cline { 2 - 5 } \multicolumn{1}{c|}{} & $1960-1969$ & $1970-1981$ & $\mathbf{1 9 8 2}-\mathbf{1 9 9 2}$ & $1960-1992$ \\
\hline Konsum' & $60,8 \%$ & $64,2 \%$ & $\mathbf{6 6 , 9} \%$ & $64,1 \%$ \\
\hline
\end{tabular}

1: prozentualer Anteil der realen Konsumausgaben in Anteilen am realen Bruttoinlandsprodukt in Preisen von 1987

Quelle: Economic Report of the President, Februar 1994, S. 287 und eigene Berechnungen.

\subsubsection{Arbeitsangebot, Arbeitsmarkt und Einkommensentwicklung}

.. If supply-side economics has validity, these dramatic cuts in marginal tax should have had a significant impact on labor supply, inducing workers to substitute work for leisure." 92

Entgegen den Erwartungen der Supply-Sider hat sich das Arbeitsangebot in der Zeit der Reaganomics verringert.

Die durchschnittliche Wochenarbeitszeit pro Beschäftigtem lag im Jahr 1981 bei 35,2 Stunden und sank bis 1992 auf 34,4 Stunden. ${ }^{93}$

Die Reaganomics konnte nicht bestätigen, daß eine Senkung der Grenzsteuersätze die Leistungsbereitschaft der Menschen erhöht.

Tabelle 25: Entwicklung des Arbeitsmarktes in den Vereinigten Staaten 1960 bis 1969. $1970-1981$. 1960 - 1992 und in der Zeit der Reaganomics 1982 bis 1992. in \%

\begin{tabular}{|l|c|c|c|c|}
\cline { 2 - 5 } \multicolumn{1}{c|}{} & $1960-1969$ & $1970-1981$ & $\mathbf{1 9 8 2}-\mathbf{1 9 9 2}$ & $1960-1992$ \\
\hline Arbeitslosenquote & $4,8 \%$ & $6,4 \%$ & $\mathbf{7 , 1} \%$ & $6,1 \%$ \\
\hline $\begin{array}{l}\text { Beschäftigungs- } \\
\text { Entwicklung }\end{array}$ & $1,8 \%$ & $2,2 \%$ & $\mathbf{1 , 5 \%}$ & $1,8 \%$ \\
\hline
\end{tabular}

1: durchschnittliche jährliche Arbeitslosenquote

2: durchschnittlicher jährlicher prozentualer Beschäftigungszuwachs

Quelle: Economic Report of the President, Februar 1994, S. 306 und eigene Berechnungen.

91 Vgl. Malabre, A. L., Ungehörte Propheten: eine Insider Geschichte der modernen Ökonomie. Stuttgart 1994, S. 245.

92 Vgl. Akhtar, M.A., Harris, E.S., The Supply-Side Consequence of U.S. Fiscal Policy in the 1980s, in: Federal Reserve Bank of New York, Quarterly Review, Vol. 17, Nr.1, 1992. S. 12.

$93 \mathrm{Vgl}$. Economic Report of the President, Februar 1994, Table B-45., S. 320. 
Die Arbeitslosenquote erreichte während der Reaganomics im Durchschnitt ein Niveau von 7,1\% und lag somit über dem Durchschnitt der Jahre 1970 bis 1981 mit einer Arbeitslosenrate von 6,4\% (Tabelle 25 und Graphik 15).

Die Beschäftigungsentwicklung lag mit einem durchschnittlichen Zuwachs von 1,5 $\%$ in dem Zeitraum der Reaganomics unter den Zuwachsraten der übrigen Perioden.

Die Steuersenkungen haben nicht zu verstärkten ökonomischen Anreizen geführt, so daß sich das Arbeitsangebot bemerkenswert ausgeweitet hätte. Die Elastizität des Arbeitsangebots auf Steuersenkungen muß in Wirklichkeit wohl weniger ausgeprägt sein, als die, die Supply-Sider in Theorieannahmen zugrunde legten. ${ }^{94}$

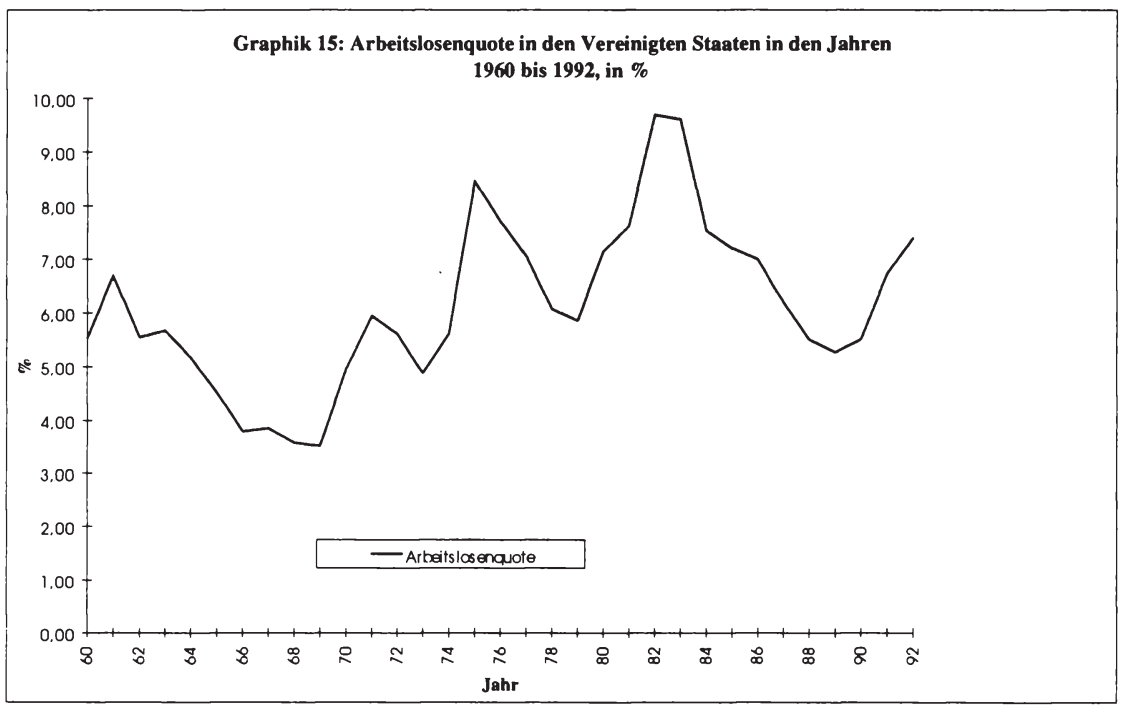

Quelle: Economic Report of the President, Februar 1994, S. 306.

Bemerkenswert ist, daß die Einkommenszuwächse in dieser Zeit zudem schwach ausgefallen sind. Eine amerikanische Familie verfügte im Jahr 1981 über ein durchschnittliches reales Jahreseinkommen von 34.862 Dollar. Im Jahr 1992 standen ihr 36.812 Dollar zur Verfügung. Bei einem männlichen Beschäftigten, der über das ganze Jahr arbeitete, ging der Verdienst sogar von 32.221 Dollar im Jahr 1981 auf 31.012 Dollar im Jahr 1992 zurück. ${ }^{95}$

94 Vgl. Kurz, R., Angebotsorientierte Wirtschaftspolitik in den USA: Grundlagen, Praxis und Konsequenzen, Tübingen 1993, S. 128.

95Vgl. Economic Report of the President, Februar 1994,, Table B-31., S. 304. 
Die männlichen Beschäftigten mußten in den 80er Jahren reale Einkommensrückgänge hinnehmen und lagen mit ihrem realen Verdienst im Jahr 1992 etwa auf dem Niveau des Jahres 1970.

Der reale Stundenlohn sank für alle Arbeitnehmer von 7,69 Dollar (1981) auf auf 7,42 Dollar (1992) und lag damit annähernd so hoch wie im Jahr 1964. ${ }^{96}$

Die 80er Jahre waren für viele Amerikaner eine Dekade der realen Einkommensrückgänge; sie waren für viele Amerikaner aber auch ein Jahrzehnt der Armut. Im Jahr 1992 lagen 36,9 Millionen Menschen mit ihrem Jahreseinkommen in den U.S.A. unter der Armutsgrenze, im Jahr 1981 waren dies noch 31,8 Millionen Menschen gewesen. ${ }^{97}$

Außerdem war die wirtschaftliche Entwicklung der 80er Jahre in den Vereinigten Staaten durch eine Einkommensumverteilung gekennzeichnet. Nach Berechnungen des Council of Economic Advisers verfügten die reichsten 5 Prozent der amerikanischen Familien im Jahr 1977 über einen Anteil von 18,6 \% am Einkommen nach Steuern, bis zum Jahr 1990 erhöhte sich dieser Anteil auf 24,5\%. Hingegen fiel dieser Anteil für die ärmsten 20 Prozent der Familien von 5,7 \% im Jahr 1977 auf 4,3 \% im Jahr 1990. ${ }^{48}$

Für diese Entwicklung hatte der Council of Economic Advisers folgende Erklärung:

„Part of this change was due to the cuts in taxes and social spending of early 1980s, the net benefits of which were heavily skewed toward the rich. But there was a much more powerful force at work. one not attribute to fiscal policy: The distribution of wage rates grew substantially more unequal. In real terms. wages at the bottom of distribution fell while wage at the top rose. "99

Die Reaganomics hat zu einer zunehmenden Spaltung bei der Einkommensentwicklung innerhalb der amerikanischen Bevölkerung geführt. Die Steuersenkungen und die Kürzungen bei den Sozialleistungen haben bei einer ungleichen Lohnentwicklung für Niedrig- und Topverdiener dazu geführt, daß die Reichen reicher und die Armen ärmer wurden. ${ }^{100}$

\subsubsection{Haushaltsentwicklung}

Die Haushaltsentwicklung des Bundes war in den Vereinigten Staaten in der Zeit der Reaganomics durch das hohe Budgetdefizit gepägt (Tabelle 26). Das Budgetdefizit war im Zeitraum von 1982 bis 1992 durchschnittlich mehr als doppelt so hoch wie im Zeitraum von 1970 bis 1981 . Hieraus resultierte auch, daß die Zinsausgaben mit einem Anteil von 12,8\% den Ausgabenspielraum des Bundes stark einengten. Ein Ziel der Reaganomics war u.a. eine geringere Staatsquote. Das Gegenteil wurde erreicht, die Staatsausgaben erreichten im Durchschnitt einen Anteil von 23,6\% am Bruttoinlandsprodukt.

96 Ebenda, Table B-45., S. 320.

97 Ebenda, Table B-31., S. 304.

98 Ebenda, S. 25.

99 Ebenda.

100 Vgl. DIE ZEIT, Das Ende der Mittelklasse, Nr. 18, vom 26. April 1996, S. 20. 
Tabelle 26: Entwicklung des Bundeshaushaltes in den Vereinigten Staaten, 1960 bis 1969, 1970 1981, 1960 - 1992 und in der Zeit der Reaganomics 1982 bis 1992, in \%

\begin{tabular}{|c|c|c|c|c|}
\hline & $1960-1969$ & $1970-1981$ & $1982-1992$ & $1960-1992$ \\
\hline Einnahmen ${ }^{1}$ & $18,9 \%$ & $19,6 \%$ & $19,7 \%$ & $19,4 \%$ \\
\hline Ausgaben $^{2}$ & $19,1 \%$ & $21,4 \%$ & $23,6 \%$ & $21,5 \%$ \\
\hline Budgetdefizit $^{3}$ & $0,2 \%$ & $1,8 \%$ & $3,9 \%$ & $2,0 \%$ \\
\hline Zinsausgaben ${ }^{4}$ & $6,5 \%$ & $7,2 \%$ & $12,8 \%$ & $8,9 \%$ \\
\hline Bundeszuschüsse $^{5}$ & $8,7 \%$ & $14,5 \%$ & $10,5 \%$ & $11,5 \%$ \\
\hline $\begin{array}{l}\text { Verteidigungsausgaben - } \\
\text { Bundeshaushalt }\end{array}$ & $44,3 \%$ & $26,9 \%$ & $25,5 \%$ & $31,7 \%$ \\
\hline $\begin{array}{l}\text { Verteidigungsausgaben - } \\
\text { Bruttoinlandsprodukt }\end{array}$ & $8,5 \%$ & $5,7 \%$ & $6,0 \%$ & $6,7 \%$ \\
\hline Sozialausgaben $^{8}$ & $25,8 \%$ & $38,1 \%$ & $40,1 \%$ & $35,0 \%$ \\
\hline
\end{tabular}

1: Bundeseinnahmen in prozentualen Anteilen am nominalen Bruttoinlandsprodukt

2: Bundesausgaben in prozentualen Anteilen am nominalen Bruttoinlandsprodukt

3: Budgetdefizit des Bundes in prozentualen Anteilen am nominalen Bruttoinlandsprodukt

4: Zinsausgaben des Bundes in prozentualen Anteilen am nominalen Bruttoinlandsprodukt

5: Bundeszuschüsse an die Länder und Gemeinden in prozentualen Anteilen am Bundeshaushalt

6: Verteidigungsausgaben des Bundes in prozentualen Anteilen am Bundeshaushalt

7: Verteidigungsausgaben des Bundes in prozentualen Anteilen am nominalen Bruttoinlandsprodukt

8: Sozialausgaben des Bundes in prozentualen Anteilen am Bundeshaushalt

Quelle: National Income and Product Accounts of the United States, Volume 2. 1959-88, Economic Report of the President, Februar 1994, eigene Berechnungen.

Diese haushaltspolitische Schmach für die Supply-Sider resultierte aus dem mangelnden politischen Willen, die Ausgabenseite des Budgets zu begrenzen. Die Einnahmen des Bundes blieben in Anteilen am Bruttoinlandsprodukt mit 19,7\% in etwa auf der Höhe der Jahre 1970 bis 1981. Die Verteidigungsausgaben erhöhten sich in Anteilen am Bruttoinandsprodukt in den Jahren 1982 bis 1992 sogar auf 6,0\% im Vergleich zu 5,7 \% in der Vorperiode. Die Erhöhung der Sozialausgaben konnte trotz massiver Kürzungsprogramme aufgrund der konjunkturellen Entwicklung nicht verhindert werden. Arbeitslosigkeit und zunehmende Armut erhöhten die Sozialausgaben auf 40,1\% in prozentualen Anteilen am Bundeshaushalt. Im Vergleich zum Zeitraum von 1970 bis 1981 mit $8,2 \%$ am nominalen Bruttoinlandsprodukt stiegen die Sozialausgaben in den Jahren 1982 bis 1992 auf einen Anteil von 9,5\% an. ${ }^{101}$

101 National Income and Product Accounts of the United States, Volume 2, 1959-88, U.S. Department of Commerce und Survey of Current Business, U.S. Department of Commerce, July 1992. 
Wie bereits erläutert, haben das Budgetdefizit und der Rückgang der privaten Sparquote dazu geführt, daß bei der Finanzierung des Staatsdefizits ein zunehmender ausländischer Kapitalfluß notwendig war.

Die Tabelle 27 differenziert das Steueraufkommen des Bundes nach Einkommenund Unternehmensteuern. In Anteilen am Bruttoinlandsprodukt ist das Aufkommen aus der Einkommensteuer mit 8,4 \% in der Zeit von 1982 bis 1992 nur umwesentlich im Vergleich zur Vorperiode zurückgegangen. Hingegen hat sich der Anteil der Unternehmensteuern am Bruttoinlandsprodukt von 2,9\% (1970 bis 1981) auf 2,0 \% (1982 bis 1992) stark verringert.

Tabelle 27: Entwicklung der Steuereinnahmen des Bundes und der Bundesstaaten und der Gemeinden in den Vereinigten Staaten, 1960 bis 1969, 1970-1981, 1960 - 1992 und in der Zeit der Reaganomics 1982 bis 1992, in \%

\begin{tabular}{|l|c|c|c|c|}
\cline { 2 - 5 } \multicolumn{1}{c|}{} & $1960-1969$ & $1970-1981$ & $\mathbf{1 9 8 2}-\mathbf{1 9 9 2}$ & $1960-1992$ \\
\hline $\begin{array}{l}\text { Einkommensteuern - } \\
\text { Bund' }\end{array}$ & $8,0 \%$ & $8,5 \%$ & $\mathbf{8 , 4} \%$ & $8,3 \%$ \\
\hline $\begin{array}{l}\text { Unternehmensteuern - } \\
\text { Bund }\end{array}$ & $4,0 \%$ & $2,9 \%$ & $\mathbf{2 , 0} \%$ & $2,9 \%$ \\
\hline $\begin{array}{l}\text { Einnahmen der Bundes- } \\
\text { staaten und Gemeinden } \\
\text { aus Einkommen- und Un- } \\
\text { ternehmensteuern }\end{array}$ & $1,5 \%$ & $2,5 \%$ & $\mathbf{2 , 9 \%}$ & $2,3 \%$ \\
\hline
\end{tabular}

1: Einkommensteueraufkommen des Bundes in prozentualen Anteilen am Bruttoinlandsprodukt

2: Unternehmensteueraufkommen des Bundes in prozentualen Anteilen am Bruttoinlandsprodukt

3: Einnahmen der Bundesstaaten und Gemeinden aus Einkommen- und Unternehmensteuern in prozentualen Anteilen am nominalen Bruttoinlandsprodukt

Quelle: National Income and Product Accounts of the United States, Volume 2, 1959-88, eigene Berechnungen.

Die Bundeszuschüsse an die Bundesstaaten und Gemeinden wurden in der Zeit der Reaganomics erheblich gekürzt. Im Durchschnitt lag ihr Anteil bei 10,5\% der Gesamtausgaben des Bundes; von 1970 bis 1981 waren es noch 14,5\% gewesen (Tabelle 26). Diese Kürzungen der Finanzzuweisungen haben die Bundesstaaten und Gemeinden durch eigene Steuererhöhungen kompensiert. Der Anteil der Einkommen- und Unternehmensteuern am Bruttoinlandsprodukt, der von den Bundesstaaten und Gemeinden beansprucht wurde, erhöhte sich von 2,5 \% (1970 bis 1981) auf 2,9\% in den Jahren der

Volume 72, Number 7 und Survey of Current Business, U.S. Department of Commerce. March 1993, Volume 73, Number 3 und eigene Berechnungen. 
Reaganomics. Die Steuerpolitik der untergeordneten Gebietskörperschaften stand während der Reaganomics entgegen der Ausrichtung der Steuerpolitik und Finanzpolitik des Bundes. Der Bund senkte die Steuern und kürzte die Finanzzuweisungen an die Bundesstaaten und Gemeinden; fast zwangsläufig waren die untergeordneten Gebietskörperschaften dadurch gezwungen, ihre Steuern zu erhöhen. ${ }^{102}$

\subsubsection{Wirtschaftliche Rahmenbedingungen}

Die wirtschaflichen Rahmenbedingungen wurden langfristig, wie gezeigt, durch die Geldpolitik der Federal Reserve Bank bestimmt. ${ }^{103}$

Langfristig war die Geldpolitik der amerikanischen Zentralbank in den 80er Jahre darauf ausgerichtet, die Inflationsrate zu stabilisieren. Dies ist ihr gelungen, die durchschnittliche Inflationsrate, gemessen am Konsumentenpreisindex, war im Zeitraum von 1982 bis 1992 fast um die Hälfte niedriger als von 1970 bis 1981 (Tabelle 28). Bei diesem Vergleich muß jedoch berücksichtigt werden, daß die inflationäre Entwicklung in den U.S.A. in den 70er Jahren durch zwei exogene Preisschocks, die beiden Ölkrisen, beeinflußt war.

Die Zinspolitik der amerikanischen Zentralbank war zunächst stark restriktiv ausgelegt. Im Zeitraum von 1981 bis 1984 betrugen die kurzfristigen Zinsen im Durchschnitt 12,0\%; im Zeitraum von 1985 bis 1988 sanken sie auf 7,3\% und von 1989 bis 1992 gingen sie weiter auf $6,6 \%$ zurück. 104

Tabelle 28: Zinsentwicklung und Inflation in den Vereinigten Staaten, 1960 bis 1969, 1970 - 1981, 1960 - 1992 und in der Zeit der Reaganomics 1982 bis 1992, in \%

\begin{tabular}{|c|c|c|c|c|}
\hline & 1960-1969 & $1970-1981$ & 1982-1992 & $1960-1992$ \\
\hline $\begin{array}{l}\text { Federal Funds Ra } \\
\text { te }^{\prime}\end{array}$ & $4,2 \%$ & $8,4 \%$ & $7,9 \%$ & $7,0 \%$ \\
\hline Long Term Rate & $4,7 \%$ & $8,4 \%$ & $9,5 \%$ & $7,6 \%$ \\
\hline Inflation" & $2,4 \%$ & $7,9 \%$ & $4,0 \%$ & $4,9 \%$ \\
\hline
\end{tabular}

1: durchschnittlicher jährlicher Geldmarktzins für Tagesgeld (Jahresdurchschnitt)

2: durchschnittlicher jährlicher Zinssatz für festverzinsliche Staatsanleihen mit einer Laufzeit zwischen 10 und 30 Jahren (Jahresdurschnitt)

3: durchschnittliche jährliche Veränderungsrate des Konsumentenpreisindexes

Quelle: Economic Report of the President, Februar 1994, S. 340 und S. 353.

102 Vgl. Müller-Oestreich, K., Der Einfluß der Finanzpolitik auf die Investitionsentwicklung in den USA unter Ronald Reagan, Berlin 1992, S. 105-106.

$103 \mathrm{Vgl}$. Kapitel 3.5 der vorliegenden Ausarbeitung.

104 Vgl. Economic Report of the President, Februar 1994, Table B-72., S. 353 und eigene Berechnungen. 
Dies hing zum einen mit einer geänderten Notenbankpolitik zusammen, zum anderen aber auch mit den außenwirtschaftlichen Erfordernissen, die aufgrund des hohen Dollarkurses das Handelsbilanzdefizit der Vereinigten Staaten anwachsen ließen.

Der US-Dollar verlor gegenüber den Währungen der großen Industriestaaten während der Reaganomics an Wert. Die Tabelle 29 zeigt repräsentativ für diese Entwicklung den DM/Dollar - Wechselkurs, der in der Zeit von 1972 bis 1981 im Durchschnitt bei

2,37 DM lag und in den Jahren von 1982 bis 1992 nur noch 2,11 DM betrug. Das Handelsbilanzdefizit verdoppelte sich fast in dieser Zeit und erreichte ein Niveau von $1,1 \%$ am Bruttoinlandsprodukt (Tabelle 29).

Tabelle 29: Entwicklung des Außenhandels und des Dollarkurses in den Vereinigten Staaten. 1972 bis 1981 und in der Zeit der Reaganomics 1982 bis 1992, in \% und DM

\begin{tabular}{|l|c|c|}
\hline & $1972-1981$ & $\mathbf{1 9 8 2 - 1 9 9 2}$ \\
\hline $\begin{array}{l}\text { Handeslsbilanz- } \\
\text { Defizit' }\end{array}$ & $-0,6 \%$ & $-\mathbf{1 , 1} \%$ \\
\hline $\begin{array}{l}\text { DM/US-Dollar } \\
\text { Wechselkurs }\end{array}$ & $2,37 \mathrm{DM}$ & $\mathbf{2 , 1 1} \mathrm{DM}$ \\
\hline
\end{tabular}

1: Handelsbilanzsaldo in prozentualen Anteilen am nominalen Bruttoinlandsprodukt

2: durchschnittlicher Wechselkurs der Deutschen Mark für einen US-Dollar

Quelle: Economic Report of the President, Februar 1994, eigene Berechnungen.

Abschließend bleibt festzuhalten, daß die langfristige ökonomische Analyse der Auswirkungen der Reaganomics im Vergleich zu den Referenzperioden folgende Ergebnisse gebracht hat:

- geringes Wirtschafts- und Produktivitätswachstum

- sinkende Investitions- und Sparquoten

- hohe Arbeitslosigkeit

- schwächerer Beschäftigungszuwachs

- höhere Staatsausgaben

- wachsendes Budgetdefizit

- Einkommensumverteilung zulasten der unteren und zugunsten der oberen Einkommensgruppen

- sinkendes Arbeitsangebot

- Abwertung des Außenwertes des US-Dollars

- hohes Handelsbilanzdefizit

- moderate Inflation

Die ökonomischen Ergebnisse der Reaganomics sind keineswegs überzeugend, sie brachten, abgesehen von der inflationären Entwicklung, schlechtere Ergebnisse als die Zeit der nachfrageorientierten Wirtschaftspolitik in den 70er Jahren. 
Die weltwirtschaftlichen Rahmenbedingungen veränderten sich in den 80er Jahren. Die hohen Wachstumsraten der Volkswirtschaften der Industrienationen gehörten der Vergangenheit an. Mit einer durchschnittlichen realen Wachstumsrate des Bruttoinlandsprodukts von 2,5\% im Zeitraum von 1985 bis 1992 lag die U.S.A. über den Zuwachsraten in Deutschland $(1,9 \%)$, England $(1,7 \%)$ und Frankreich $(1,7 \%)$; nur Japan konnte ein höheres Wirtschaftswachstum mit durchschnittlich 3,2\% in dieser Zeit verzeichnen. ${ }^{105}$

Im internationalen Vergleich hat die Reaganomics hinsichtlich des Wirtschaftswachstums ein gutes Ergebnis erzielt, das relativiert ein wenig die schlechten wirtschaftlichen Resultate der langfristigen Analyse auf nationaler Ebene.

\subsection{Resümee}

"Die gesamte Konzeption war in der Tat falsch angelegt. Die grundlegenden Annahmen und die fiskalpolitische Architektur haben überhaupt erst jene Dummheiten heraufbeschworen, die unsere ökonomische Steuerungsfähigkeit heute blockieren.

Die Reagan-Revolution war radikal, unbesonnen und arrogant." 106

Die Reaganomics war ein überaus ambitioniertes wirtschaftspolitisches Programm.

Ein Wirtschaftsprogramm, das jedoch aufgrund der konjunkturellen Krise in den Vereinigten Staaten zu Beginn der 80er Jahre unter Startschwierigkeiten litt. Steuersenkungsprogramme, Staatsausgabenkürzungen und Deregulierungen sollten dazu führen, $\mathrm{da} ß \mathrm{mehr}$ investiert, gespart und gearbeitet werde. Doch das angebotsorientierte Wirtschaftskonzept hatte, wie gezeigt, keines dieser Ziele erreicht und dabei die Auswirkungen der Geldpolitik auf die Wirtschaftsentwicklung erheblich unterschätzt.

Die Zentralbank erhöhte am Anfang der 80er Jahre die Zinssätze stark, um die hohe Inflation zu bekämpfen. Die Konsequenzen waren fatal: die hohen Zinssätze wirkten kontraproduktiv auf die Investitionsentwicklung, der Dollarkurs stieg stark an und in Folge erhöhte sich das Handelsbilanzdefizit. Erst Mitte der 80er Jahre senkte die Federal Reserve Bank die Zinsen und in Folge verlor der Dollar gegenüber den Währungen der wichtigsten Handelspartner der Vereinigten Staaten erheblich an Wert.

Hinzu kam, daß die schwache Konjunktur die Steuereinnahmen sinken ließ und die Regierung im Verlauf der 80er Jahre dazu zwang, teilweise Steuersenkungen wieder rückgängig zu machen.

Außerdem wurden entgegen des ursprünglichen Ansatzes der Reaganomics und der Supply-Side Theorie die Bundesausgaben weiter erhöht. Diese Finanzpolitik führte da$\mathrm{zu}$, daß das Budgetdefizit stark anstieg. Im Zusammenhang mit dem amerikanischen Handelsbilanzdefizit sprach man nunmehr von einem Zwillingsdefizit.

105 Vgl. Economic Report of the President, Februar 1994, Table B-111., S. 395 und eigene Berechnungen.

106 Vgl. Stockman, D., Der Triumph der Politik, Die Krise der Reagan-Regierung und ihre Auswirkung auf die Weltwirtschaft, München 1986, S. 426. 
Im Verlauf der beiden Amtszeiten Ronald Reagans lagen die jährlichen Budgetdefizite des Bundeshaushaltes so hoch wie nie zuvor in der Geschichte der Vereinigten Staaten. Im Jahr 1980, zu Beginn der Amtszeit Ronald Reagans, betrug der Schuldenstand des Bundes 908,5 Mrd. Dollar, am Ende seiner zweiten Amtszeit im Jahr 1988 hatte die Schuldenlast eine Höhe von 2.600,8 Mrd. Dollar erreicht. Als Bush sein Präsidentschaftsamt im Jahr 1992 abgab, waren die Schulden mittlerweile bei 4.001,9 Mrd. Dollar angelangt. ${ }^{107}$ Auf dem Hintergrund der Tatsache, daß die Reaganomics das Zeitalter des „deficit spending“ ablösen und das Staatsdefizit reduzieren wollte, hat die angebotsorientierte Wirtschaftspolitik in den Vereinigten Staaten ihr Ziel weit verfehlt. Im Zeitraum von 12 Jahren, von 1980 bis 1992, wurden die Schulden des Bundeshaushaltes vervierfacht.

Die Projektion für die geplanten Steuereinnahmen und die Entwicklung des Budgetdefizits, die dem Wirtschaftsprogramm „A Program for Economic Recovery“ von Ronald Reagan zu entnehmen war, belegten, daß die Annahmen der Laffer-Kuve hinter den Vorstellungen der Reaganomics standen. Das Wirtschaftswachstum sollte dazu führen, daß sich bei gleichzeitigen Ausgabenkürzungen die Steuersenkungen quasi selbstfinanzieren und in einem Zeitraum von drei Jahren das Budgetdefizit in ein Budgetüberschuß verwandelt wird. ${ }^{108}$

Es erscheint deshalb geradezu grotesk, wenn Verfechter der Supply-Side Policy im Nachhinein behaupten, da $B$ die Angebotspolitik nicht auf das Ziel hin angelegt war, dem Staat höhere Einnahmen zu verschaffen oder den Ausgleich des Budgets zu bewirken. 109

Die Laffer-Annahmen traten, wie im 3. Kapitel gezeigt, nicht ein. Die Steuersenkungen verstärkten die finanzpolitischen Probleme und gaben keine Antwort darauf, wie die Wachstumsschwäche der amerikanischen Wirtschaft behoben werden könne.

Ein weiteres Ergebnis des 3. Kapitels war, daß die Finanzzuweisungen des Bundes an die untergeordneten Gebietskörperschaften in der Zeit der Reaganomics erheblich gesenkt wurden. Diese Entwicklung veranlaßte die Bundesstaaten und Kommunen, ihrerseits die Steuern in dieser Zeit zu erhöhen. Ein Teil der Steuersenkungen, die der Bund beschlossen hatte, wurde durch die Steuererhöhungen der Bundesstaaten und Gemeinden neutralisiert.

Bill Clinton, der als 42. Präsident der Vereinigten Staaten die Reaganomics beendete, stellte am 14. Februar 1994 fest:

107 Vgl. Economic Report of the President, Februar 1994, Table B-77., S. 359.

108 Vgl. The White House, Office of the Press Secretary: A Program for Economic Recovery, Washington D.C., February 18, 1981.

$109 \mathrm{Vgl}$. Roberts, P. G., Angebotsorientierte Wirtschaftspolitik, theoretische Grundlagen und politische Erfolge: Eine Bewertung der amerikanischen Erfahrungen in den achtziger Jahren, in: Zeitschrift für Wirtschaftspolitik, Jg. 38 (1989), Heft 1, S. 6-7. 
. For 12 years a policy of trickle-down economies built a false prosperity on a mountain of Federal debt. As a result of our national drift, far too many American families, even those with two working parents, no longer dream the American dream of a better life for their children." 110

Diese Einschätzung von Bill Clinton ist wahrscheinlich der wichtigste Grund dafür, daß die Reaganomics im Jahr 1992 endete. Das 3. Kapitel zeigte, daß viele Amerikaner in den Jahren der angebotsorientierten Wirtschaftspolitik reale Einkommensverluste hinnehmen mußten. Das Auseinanderdriften der amerikanischen Gesellschaft nahm in dieser Zeit an Geschwindigkeit zu, was dazu führte, daß die Armut in den Vereinigten Staaten größer wurde und die hohen Einkommensbezieher überproportional stark von den Steuersenkungen profitierten. ${ }^{111}$

David Stockman, der zu Beginn der Amtszeit Reagans das Haushaltsbüro leitete, umschrieb das Scheitern der Supply-Side Policy mit der Formulierung:" Der Triumph der Politik" und widmete dieser Aussage ein ganzes Buch. ${ }^{12}$ Stockmann vertritt die Auffassung, daß eine politische Klasse, die ausschließlich von Wahl zu Wahl denkt, gleichsam den Untergang für jede langfristig konzipierte Wirtschaftspolitik bedeutet; somit war auch die angebotsorientierte Wirtschaftspolitik in den U.S.A. zum Scheitern verurteilt. Für Stockman waren die Vertreter der Reaganomics letztendlich Wohlfahrtspolitiker, die nur halbe Revolutionäre waren. Sie waren zwar bereit die Steuern zu senken, aber ihnen fehlte der Mut, für Ausgabensenkungen zu sorgen. Stockman kommt zu dem Schluß, daß Ronald Reagan von seinen Beratern und seinen eigenen Illusionen dazu gebracht worden war, sich eines der riskantesten und unverantwortlichsten Wirtschaftsprogramme aller Zeiten zu eigen zu machen. ${ }^{113}$

Eines hat die Supply-Side Policy und die Reaganomics auf jeden Fall gezeigt: ökonomische Konzepte und wirtschaftspolitische Praxis müssen, wenn sie denn funktionieren sollen, aufeinander abgestimmt werden.

Die Reaganomics war einem schwierigen Policy-Mix aus unkontrollierter Fiskalpolitik und restriktiver Geldpolitik, begleitet von binnen- und außenwirtschaftlichen Problemen, ausgesetzt. Für diese Entwicklung war aber auch das Theoriegebäude der Angebotspolitik verantwortlich, die den Wirtschaftspolitikern versprach, daß Steuersenkungen die Wirtschaft ankurbeln und den Haushalt sanieren würden. Dieser Wirkungszusammenhang blieb in den Vereinigten Staaten aus, und die Abstimmung zwischen Steuer-, Ausgaben- und Geldpolitik funktionierte nicht.

Im Fall der Reaganomics hat sowohl die Theorie der Angebotspolitik als auch die politische Praxis, die mit der Umsetzung betraut war, keine überzeugende Leistung erbracht.

110 Vgl. Economic Report of the President, Februar 1994, S. 3.

111 Vgl. Kurz, R.. Angebotsorientierte Wirtschaftspolitik in den USA: Grundlagen. Praxis und Konsequenzen, Tübingen 1993, S. 191-192.

112 Vgl. Stockman, D., Der Triumph der Politik, Die Krise der Reagan-Regierung und ihre Auswirkung auf die Weltwirtschaft. München 1986.

113 Ebenda. S. 400-445. 
4 Schätzung des marginalen Steuersatzes für die U.S.A.

Die Supply-Sider argumentierten, daß die Steuern so strukturiert werden, daß sie den größtmöglichen Einfluß auf die Investitions-, Spar- und Arbeitshöhe haben sollten.

Hieraus folgerten die Angebotstheoretiker, daß über eine Senkung des marginalen Steuersatzes der "Tradeoff" zwischen Arbeit und Freizeit, Investitionen und Konsum erhöht werden könne. ${ }^{1}$

Die Reaganomics setzte die Vorstellungen der Supply-Side Policy in die Praxis um. In den 80er Jahren wurden, wie gezeigt, in den Vereinigten Staaten die Steuern gesenkt.

Die Laffer-Kurve gab in diesem Zusammenhang Argumentationshilfe. Der graphische Verlauf der Kurve zeigte, daß es zwei Steuersätze gebe, welche die gleichen Steuereinnahmen ergeben würden; ein hoher Steuersatz aufgrund der Grundlage einer niedrigen Bemessungsgrundlage und ein niedriger Steuersatz auf der Grundlage einer hohen Bemessungsgrundlage. ${ }^{2}$ Die Senkung der Grenzsteuersätze sollte das Wirtschaftswachstum erhöhen und den durchschnittlichen Steuersatz konstant halten. Die Voraussetzung für diese Entwicklung ist, daß der durchschnittliche Steuersatz konstant gehalten werden kann, wenn sich die Steuereinnahmen und das Bruttoinlandsprodukt mit der gleichem Wachstumsrate verändern. Dieser Zusammenhang ergibt sich aus der Berechnung des durchschnittlichen Steuersatzes, der sich aus der Division der Steuereinnahmen durch die Bemessungsgrundlage, z.B. das Bruttoinlandsprodukt, ergibt.

Das Kapitel 3 der Ausarbeitung zeigt, da $B$ die wirtschaftlichen Ergebnisse der Reaganomics dem Anspruch der Supply-Side Policy nicht gerecht wurden. Wirtschaftswachstum, Investitionen und das Sparaufkommen gingen im langfristigen Durchschnitt im Vergleich zu den Vorperioden zurück.

Aus den ökonomischen Ergebnissen der Reagonomics müßte, sofern man der Supply-Side Policy Gültigkeit einräumt, gefolgert werden, daß der marginale Steuersatz nicht gesenkt werden konnte.

Koester und Kormendi haben für den Zeitraum der 70er Jahre die marginalen und durchschnittlichen Steuersätze für 63 Länder geschätzt. ${ }^{3}$

Die marginale Steuerquote wurde dabei über die Regression der jeweiligen gesamten Steuereinnahmen eines Landes in Bezug zum nominalen Bruttoinlandsprodukt ermittelt. ${ }^{4}$

1 Vgl. Bartlett, B. R., Reaganomics: supply-side economics in action, Westport 1981, S. 6.

2 Ebenda, S. 14.

3 Vgl. Koester, R. B., Kormendi. R. C., Taxation, Aggregate Activity and Economic Growth: Cross-Country Evidence on some Supply-Side Hypothesis, Economic Inquiry 27. S. 367-386.

4 Ebenda, S. 370. 
Der durchschnittliche Steuersatz ergab sich bei dieser Untersuchung aus dem Anteil der Steuereinnahmen am Bruttoinlandsprodukt.

Koester und Kormendi gingen der Hypothese der Supply-Side Theoretiker nach, daß ein Konstanthalten des durchschnittlichen Steuersatzes bei einer gleichzeitigen Reduzierung des marginalen Steuersatzes das Wachstum der Wirtschaft positiv beeinflussen würde. Für die Vereinigten Staaten schätzen sie z.B. eine marginale Steuerquote von $0,236(23,6 \%) .^{5}$

Aus den jeweiligen Wachstumsraten des Bruttoinlandsprodukts und den Ergebnissen für die marginalen und durchschnittlichen Steuerquoten folgerten sie, daß ein Zusammenhang zwischen diesen Steuersätzen und der Höhe des Wirtschaftswachstums besteht. Nach ihrer Auffassung lassen die Ergebnisse den Schluß zu, daß ein konstanter durchschnittlicher Steuersatz und ein hoher (niedriger) marginaler Steuersatz eine niedrigere (höhere) Wachstumsrate der Wirtschaft bedingen. ${ }^{6}$

In einer ähnlichen Untersuchung schätzten Barro und Sahasakul die marginale Steuerquote auf der Grundlage der Höhe der Einkommensteuer der Vereinigten Staaten in den Jahren 1944 bis 1978. ${ }^{7}$ Nach ihren Ergebnissen erhöhte sich im Vergleich zu den 60er Jahren der durchschnittliche marginale Steuersatz von 0,235 (23,5\%) auf 0,268 $(26,8 \%)$ bis zum Jahr $1978 .^{8}$

Aus diesen empirischen Untersuchungen und den ökonomischen Ergebnissen der Reagonomics resultierte die Idee, den marginalen Steuersatz für die Vereinigten Staaten für die Zeiträume von 1960 bis 1992, 1960 bis 1969, 1970 bis 1981 und 1982 bis 1992 differenziert nach Gebietskörperschaften zu schätzen.

Ein Ergebnis des 3. Kapitels der Ausarbeitung war u.a., daß der Bund zur Entlastung des Haushaltes die Finanzzuweisungen an die Bundesstaaten und Gemeinden gekürzt hatte. Im Gegenzug haben die State and Local Governments die Steuern erhöht.

Die föderative Struktur der Vereinigten Staaten ermöglicht es den Bundesstaaten und Gemeinden, neben den Bundessteuern jeweils eigene Steuern zu erheben. Hierzu zählen z.B. Einkommensteuern, Verbrauchssteuern, Treibstoffsteuern und Grundsteuern. ${ }^{9}$

In diesem Zusammenhang bleibt die Frage zu klären, ob es der Reaganomics gelang, den marginalen Steuersatz für die gesamten Vereinigten Staaten ( Bund, Bundesstaaten und Gemeinden ) zu senken; denn nur unter dieser Voraussetzung könne die SupplySide Policy Wirkung erzielen.

5 Ebenda. S. 384-385.

6 Ebenda, S. 383.

7 Vgl. Barro, R. J.. Sahasakul, C., Measuring the Average Marginal Tax Rate from the Individual Income Tax, Journal of Business, vol. 56, no. 4, 1983, S. 419-452.

8 Ebenda. S. 451.

9 Vgl. Holtfrerich, C.-L.. Wirtschaft USA, München 1991, S. 287-301. 


\subsection{Ansatz für die Schätzung}

Annahme: Die Ökonomie der Vereinigten Staaten funktioniert nach den Vorstellungen der Supply-Side Theoretiker.

Konsequenz: Die marginale Steuerquote wird zur entscheidenden Referenzgröße für die gesamtwirtschaftliche Entwicklung.

Frage: Ist es der Reaganomics mit ihrer Wirtschaftspolitik gelungen, die Grenzsteuerbelastung, bei einer konstanten durchschnittlichen Steuerquote, zu senken, um damit im Sinn der Angebotsökonomen die Voraussetzung für ein nachhaltiges Wirtschaftswachstum zu schaffen?

\subsection{Vorgehen bei der Schätzung}

Zur Schätzung der marginalen Steuerquote (b) wurden die Steuereinnahmen (TAXREV) zum Bruttoinlandsprodukt (GDP) in Beziehung gesetzt. Das GDP wird hierbei als Bemessungsgrundlage für die Höhe der Steuereinnahmen verwendet.

Der marginale Steuersatz (b) - oft auch als Grenzsteuersatz bezeichnet - entspricht dem Verhältnis der marginalen Änderung der Steuereinnahmen (Steuerbetrages) bei marginaler Änderung der Bemessungsgrundlage.

Der Beobachtungszeitraum erstreckt sich vom 1. Quartal 1960 bis einschließlich dem 4. Quartal 1992.

Sowohl bei den Steuereinnahmen als auch beim Bruttoinlandsprodukt wurden nominale - zu jeweiligen Preisen ermittelte - Quartals-Werte für die Schätzung verwendet.

Die TAXREV setzen sich aus direkten Steuern (Personal Tax), indirekten Steuern (Indirect Tax) und Unternehmenssteuern (Corporate Tax) zusammen.

Die Schätzung der marginalen Steuerquote wurde zum einen für unterschiedliche Zeitintervalle $(\mathbf{t})$ als auch differenziert nach Gebietskörperschaften $(\mathbf{K})$ und deren jeweiligem Steueraufkommen (TAXREV $\mathbf{T}_{1}^{\mathrm{K}}$ ) vorgenommen.

\subsubsection{Die Regressionsgleichung}

Die Regressionsgleichung zur Schätzung der marginalen Steuerquote bestimmt sich folgendermaßen:

TAXREV $_{1}^{K}=a_{t}^{k}+b_{1}^{K} \operatorname{GDP}_{t}+e_{1}^{k}$ 
TAXREV, ${ }_{1}^{\mathrm{K}}$ : Steuereinnahmen der Gebietskörperschaft K für den Zeitraum t.

GDP $_{\mathbf{t}}$ : Bruttoinlandsprodukt der Vereinigten Staaten für den Zeitraum $\mathrm{t}$.

$\mathbf{b}_{1}{ }_{\mathrm{K}}$ : marginale Steuerquote der Gebietskörperschaft $\mathrm{K}$ für den Zeitraum $\mathrm{t}$.

$\mathbf{a}_{1}{ }^{\mathrm{K}}$ : Ordinaten-Abschnitt der Regressionsgeraden.

$\mathbf{e}_{1}^{\mathrm{K}}$ : Störterm der Schätzung.

Leitet man die Funktion für die Steuereinnahmen nach dem Bruttoinlandsprodukt ab, erhält man die marginale Steuerquote:

$\frac{\text { dTAXREV }_{t}{ }^{K}}{d G D P_{t}}=b_{t}^{K}$

Folgende Zeiträume und Gebietskörperschaften wurden untersucht: Zeiträume:

1) 1. Quartal 1960 - 4. Quartal 1992

2) 1. Quartal 1960 - 4. Quartal 1969

3) 1. Quartal 1970 - 4. Quartal 1981

4) 1. Quartal 1982 - 4. Quartal 1992

Gebietskörperschaften :

1) U.S.A. (Government: Bund, Bundesstaaten und Gemeinden)

2) Bund (Federal Government: Bund)

3) Bundesstaaten und Gemeinden (State and Local Government: Bundesstaaten und Gemeinden)

Die Regressionsgleichung wurde, um eine eventuelle Autokorrelation zu vermeiden, nach dem Cochrane-Orcutt-Verfahren geschätzt. Die Ergebnisse für die Schätzungen ergaben sich jeweils nach der zweiten Iteration des Cochrane-Orcutt-Verfahrens.

Die Tabelle 30 weist für den jeweiligen Schätzzeitraum und die jeweilige Gebietskörperschaft das Absolutglied der Schätzung $\left(\mathbf{a}_{1}^{\mathrm{K}}\right)$, die marginale Steuerquote $\left(\mathbf{b}_{1}^{\mathrm{K}}\right)$, das Bestimmtheitsmaß $\mathrm{R}^{2}$ für die Güte der Anpassung und den Durbin-Watson-Wert für die Überprüfung der Auokorrelation aus.

\subsection{Ergebnisse der Schätzung des marginalen Steuersatzes}

Zusammenfassung der Ergebnisse für: $\mathbf{a}_{1}^{\mathrm{K}}, \mathbf{b}_{\mathrm{t}}^{\mathrm{K}}, \mathbf{R}^{\mathbf{2}}$ und D.W.-Werte: 
Tabelle 30: Schätzergebnisse für die marginale Steuerquote in den Vereinigten Staaten

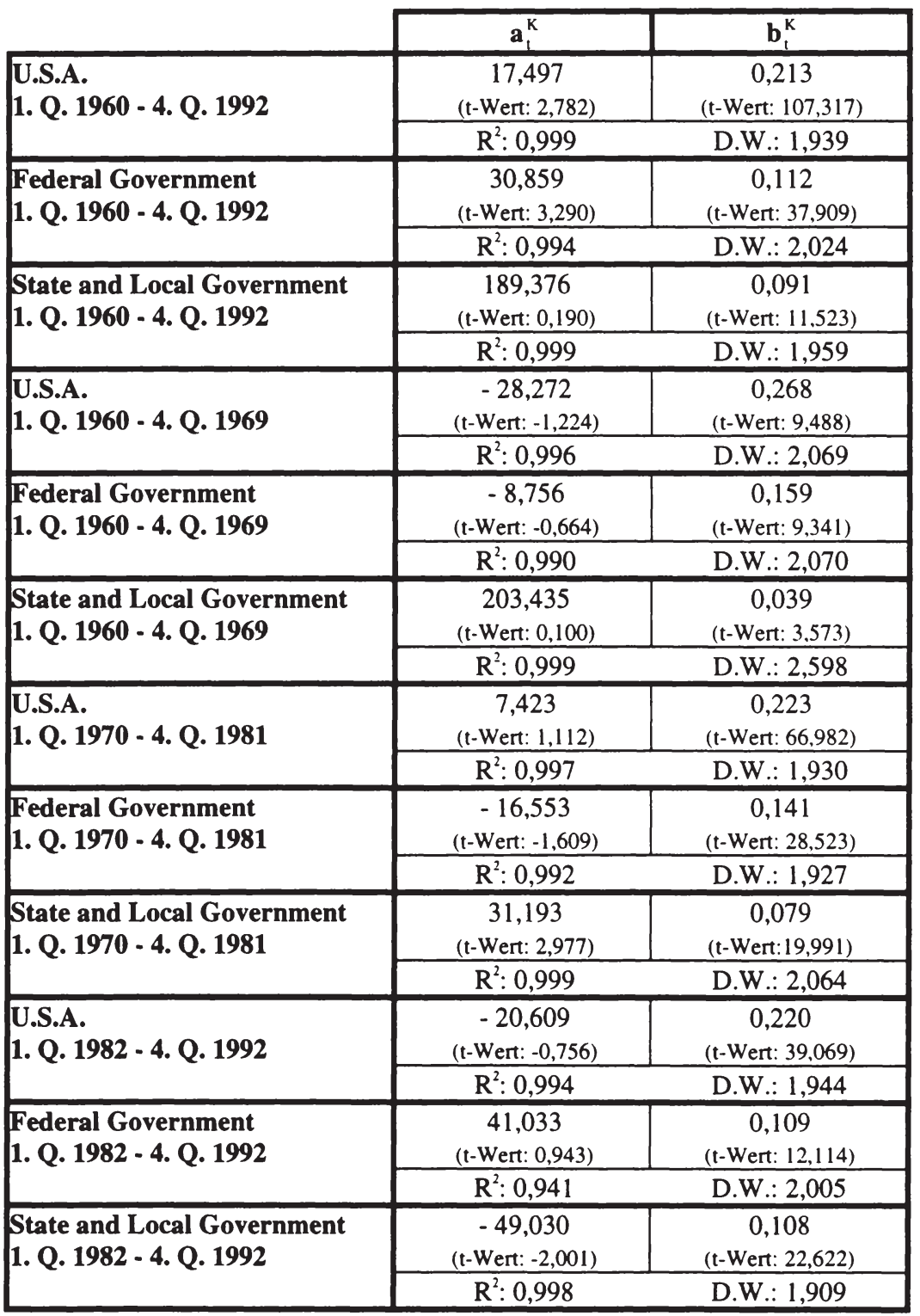


Quelle: National Income and Product Accounts of the United States, Volume 2, 1959-88. U.S. Department of Commerce und Survey of Current Business, U.S. Department of Commerce, July 1992, Volume 72, Number 7 und Survey of Current Business, U.S. Department of Commerce, March 1993, Volume 73, Number 3 und eigene Berechnungen.

Neben den marginalen Steuerquoten wurden auch die durchschnittlichen Steuerquoten $\left(\mathbf{c}_{1}^{\mathrm{K}}\right)$ der Gebietskörperschaften für die Beobachtungszeiträume bestimmt.

Die Berechnung erfolgt über die Division der Steuereinnahmen der Gebietskörperschaften durch das Bruttoinlandsprodukt für den jeweiligen Zeitraum.

Für die durchschnittliche Steuerquote gilt:

$\mathbf{c}_{\mathrm{t}}^{\mathrm{K}}=\sum_{\mathrm{r}=1}^{n}\left(\frac{\mathrm{TAXREV}_{1}^{\mathrm{K}}}{\mathrm{GDP}_{\mathrm{t}}}\right) / n \quad ; \mathrm{n}=$ Anzahl der Quartale des Beobachtungszeitraums

Tabelle 31: Die durchschnittliche Steuerquote in den Vereinigten Staaten

\begin{tabular}{|l|c|}
\cline { 2 - 2 } \multicolumn{1}{l|}{} & $c_{1}^{\mathrm{K}}$ \\
\hline 1. Q.A. 1960 - 4. Q. 1992 & 0,222 \\
\hline $\begin{array}{l}\text { Federal Government } \\
\text { 1. Q. } 1960 \text { - 4. Q. } 1992\end{array}$ & 0,135 \\
\hline $\begin{array}{l}\text { State and Local Government } \\
\text { 1. Q. } 1960 \text { - 4. Q. } 1992\end{array}$ & 0,087 \\
\hline $\begin{array}{l}\text { U.S.A. } \\
\text { 1. Q. } 1960 \text { - 4. Q. } 1969\end{array}$ & 0,236 \\
\hline $\begin{array}{l}\text { Federal Government } \\
\text { 1. Q. } 1960 \text { - 4. Q. } 1969\end{array}$ & 0,154 \\
\hline $\begin{array}{l}\text { State and Local Government } \\
\text { 1. Q. } 1960 \text { - 4. Q. } 1969\end{array}$ & 0,082 \\
\hline $\begin{array}{l}\text { U.S.A. } \\
\text { 1. Q. 1970 - 4. Q. } 1981\end{array}$ & 0,231 \\
\hline
\end{tabular}




\begin{tabular}{|l|c|}
\cline { 2 - 2 } \multicolumn{1}{c|}{} & $\mathrm{c}_{\mathrm{t}}^{\mathrm{K}}$ \\
\hline Federal Government \\
1. Q. 1970 - 4. Q. 1981 & 0,140 \\
\hline $\begin{array}{l}\text { State and Local Government } \\
\text { 1. Q. } 1970 \text { - 4. Q. } 1981\end{array}$ & 0,091 \\
\hline $\begin{array}{l}\text { U.S.A. } \\
\text { 1. Q. 1982 - 4. Q. } 1992\end{array}$ & 0,219 \\
\hline $\begin{array}{l}\text { Federal Government } \\
\text { 1. Q. } 1982 \text { - 4. Q. } 1992\end{array}$ & 0,123 \\
\hline $\begin{array}{l}\text { State and Local Government } \\
\text { 1. Q. } 1982 \text { - 4. Q. } 1992\end{array}$ & 0,097 \\
\hline
\end{tabular}

Quelle: National Income and Product Accounts of the United States, Volume 2, 1959-88, U.S. Department of Commerce und Survey of Current Business, U.S. Department of Commerce, July 1992, Volume 72, Number 7 und Survey of Current Business, U.S. Department of Commerce. March 1993, Volume 73. Number 3 und eigene Berechnungen.

\subsection{Interpretation der Ergebnisse}

Die marginale Steuerquote der U.S.A. ist im Zeitraum von 1982 bis 1992 im Vergleich zu den Jahren von 1970 bis 1981 von 0,223 (22,3\%) auf $0,220(22,0 \%)$ zurückgegangen. Die durchschnittliche Steuerquote reduzierte sich für den gleichen Zeitraum von $0,231(23,1 \%)$ auf $0,219(21,9 \%)$. In den Jahren von 1960 bis 1969 lag der Grenzsteuersatz für die gesamte U.S.A. auf einem höheren Niveau als in den Vergleichsperioden.

Die Schätzergebnisse der Regression für den Zeitraum von 1982 bis 1992 sind von besonderem Interesse. Hier zeigt sich im Vergleich mit der Vorperiode, zu welchen Ergebnissen die Reaganomics geführt hat. Die Senkungen der Steuern, die ausschließlich auf Bundessteuern bezogen waren, führten zum Absinken des Grenzsteuersatzes auf Bundesebene von 0,141 (14,1 \%) auf 0,109 (10,9\%), was einen Rückgang von 3,2 Prozentpunkten bedeutet. Dies wird durch die Schätzung belegt. Die Supply-Side Policy hat hier deutlich ihre Wirkung gezeigt. Dennoch führte sie, bezogen auf die gesamten Vereinigten Staaten, nach den Ergebnissen der Schätzung nur zu einer unbedeutenden Senkung des marginalen Steuersatzes von 0,3 Prozentpunkten von 22,3\% (1970 bis 1981) auf $22,0 \%$ (1982 bis 1992). Selbst wenn man also davon ausgeht, daß Wohl oder 
Wehe der Volkswirtschaft der U.S.A. von der marginalen Steuerquote abhängt, gelang es der angebotsorientierten Wirtschaftspolitik der Reagan- und Bush-Regierung nicht, diese signifikant zu senken.

Wie war dies möglich, der Bund hatte doch die Grenzsteuersätze massiv gesenkt. Die Antwort auf diese Frage liefern die Schätzergebnisse für die State and Local Government in den Jahren 1970 bis 1981 und 1982 bis 1992 . Im Zeitraum von 1970 bis 1981 lag der Grenzsteuersatz der Bundesstaaten und Gemeinden bei 0,079 (7,9\%), in der Zeit der Reaganomics bei 0,108 (10,8\%). Der marginale Steuersatz, der sich aus den Steuereinnahmen der Bundesstaaten und Gemeinden ergab, stieg in der Zeit der angebotsorientierten Wirtschaftspolitik um 2,9 Prozentpunkte. Der Bund senkte den marginalen Steuersatz um 3,2 Prozentpunkte und die untergeordneten Gebietskörperschaften erhöhten über Steuererhöhung den Grenzsteuersatz um 2,9 Prozentpunkte, in Folge blieb der Grenzsteuersatz für die U.S.A. (Bund, Bundesstaaten und Gemeinden) fast unverändert.

Aus der langfristigen Analyse der wirtschaftlichen Auswirkungen der Reaganomics ergab sich, daß die Finanzzuweisungen des Bundes an die untergeordneten Gebietskörperschaften im Zeitraum von 1982 bis 1992 in Anteilen am Bruttoinlandsprodukt stark zurückgingen und die Bundesstaaten und Gemeinden sich dadurch gezwungen sahen, ihrerseits wegen der Kürzungen der Bundeszuweisungen die Steuern zu erhöhen. Die Ergebnisse der langfristigen wirtschaftlichen Auswirkungen der Reaganomics werden durch die Schätzergebnisse für die marginale Steuerquote der Vereinigten Staaten bestätigt. ken.

Für die U.S.A. gelang es der Reaganomics nicht, die marginale Steuerquote zu sen-

Ebensowenig konnte der durchschnittliche Steuersatz konstant gehalten werden; er sank auf 0,219 (21,9\%), dem niedrigsten Wert im Vergleich zu allen Beobachtungszeiträumen. In diesem Zusammenhang stellt sich die Frage, ob die Reagonomics nicht gelingen konnte, weil es zu keiner Senkung der marginalen Steuerquote kam.

Richtig ist, der Bund hat die Steuern gesenkt, die Bundesstaaten und Gemeinden haben die Steuern erhöht und dem Bund kann in diesem Zusammenhang der Vorwurf gemacht werden, daß versäumt wurde, die Steuer- und Finanzpolitik in ihrer Gesamtwirkung korrekt abzuschätzen.

Falsch wäre deshalb zu meinen, die Supply-Side Policy hätte Wirkung gezeigt, wenn die marginale Steuerquote für die U.S.A. hätte gesenkt werden können.

Dies ist deshalb überaus fraglich, weil sich die Vereinigten Staaten bei einem marginalen Steuersatz von 0,223 (22,3\%) für den Zeitraum von 1970 bis $1981 \mathrm{im}$ Sinn der Laffer-Argumentation wohl nicht im prohibitiven Bereich der Besteuerung befunden haben; also rechts vom Scheitelpunkt auf der Laffer-Kurve.

Bei dieser niedrigen Höhe des Grenzsteuersatzes kann davon ausgegangen werden, daß sich die U.S.A. im normalen Bereich der Besteuerung (normal range), links vom Scheitelpunkt auf der Laffer-Kurve, befand. Das heißt, selbst wenn es der Reaganomics gelungen wäre, den Grenzsteuersatz für die U.S.A. zu senken, hätte die Supply-Side 
Policy ihre Wirkung nicht entfalten können, weil der Grenzsteuersatz schon niedrig war.

Die Schätzergebnisse von Koester und Kormendi ergaben z.B. für Schweden in den 70er Jahren einen Grenzsteuersatz von 67,8 Prozent, dies wäre eher ein Steuersatz, bei dem man es sich vorstellen könnte, daß eine Senkung des marginalen Steuersatzes den Laffer-Effekt eintreten ließe.

Die Tobin-Herleitung der Laffer-Kurve hatte den Scheitelpunkt der Laffer-Kurve sogar bei einem Steuersatz von 83,3 Prozent ermittelt.

Der Test der Supply-Side Policy durch die Reaganomics konnte auch deshalb nicht gelingen, weil das Versuchsobjekt, die Vereinigten Staaten, aufgrund des niedrigen marginalen Steursatzes hierfür denkbar ungeeignet war. 
Joachim Nagel - 978-3-631-75238-8

Downloaded from PubFactory at 01/11/2019 06:47:33AM

via free access 


\section{Ergebniszusammenfassung und Schlußbemerkung}

Die Arbeit versteht sich nicht als grundsätzliches Plädoyer gegen eine angebotsorientierte Wirtschaftspolitik. Die Schätzung der marginalen Steuerquoten für die einzelnen Zeiträume von 1960-92 zeigte, daß die Supply-Side Policy ihr Hauptziel nicht erreicht hat; die Grenzsteuersätze konnten insgesamt nicht signifikant gesenkt werden.

Hat die Supply-Side Policy grundsätzlich versagt?

Vergegenwärtige man sich noch einmal die Situation, mit der die Vereinigten Staaten in den Jahren vor dem Amtsantritt Reagans im Jahr 1981 konfrontiert war, dann läßt sich die Frage verneinen.

Kapitel 1 der Arbeit erläuterte die Ausgangssituation in den Vereinigten Staaten in dieser Zeit, die die Voraussetzung für die Verwirklichung der Supply-Side Policy bildete. Es zeigte sich, daß die bis dahin maßgeblich auf eine Stärkung der Nachfrageseite konzentrierte Wirtschaftspolitik kein Mittel gegen Inflation und Arbeitslosigkeit wußte. Der Keynesianismus stand den drängenden Problemen der amerikanischen Volkswirtschaft, bedingt durch Vietnam-Krieg, Ölkrisen und soziologische Umbrüche, mehr oder weniger ratlos gegenüber. Bei einer Berücksichtigung dieser Voraussetzungen ist es doch nur allzu verständlich, daß die Menschen und insbesondere die Politiker, die wiedergewählt werden möchten, sich für eine Wirtschaftspolitik gewinnen lassen, die eine Lösung der wirtschaftlichen Probleme verspricht.

Noé hat die damalige Situation treffend beschrieben:

„Das Versagen der Politik nach den Ölpreiskrisen Mitte der siebziger Jahre, Vollbeschäftigung bei Preisniveaustabilität und angemessenem Wirtschaftswachstum zu erreichen, hat zu einer Vertrauenskrise geführt und zu einer Grundhaltung des „Rette sich wer kann“."

Genau in dieses wirtschaftspolitische Vakuum stieß Ende der 70er Jahre die SupplySide Policy in den Vereinigten Staaten. Eine Wirtschaftspolitik, die versprach, im diametralen Gegensatz zum Keynesianismus die ökonomischen Probleme ausschließlich über eine Stärkung der Angebotsseite der Wirtschaft in den Griff zu bekommen; vornehmlich umfassende Steuersenkungen sollten diese Entwicklung möglich machen.

Diese „neue" Wirtschaftspolitik und die dazugehörige Theorie wurden im zweiten Kapitel präsentiert. Eine „neue“ Wirtschaftstheorie oder treffender eine geschickt verpackte Renaissance wesentlicher Elemente der klassischen Wirtschaftstheorie. Einige Mentoren der angebotsorientierten Wirtschaftspolitik, vor allem A. B. Laffer, verstanden es glänzend, die Grundzüge ihrer wirtschaftspolitischen Vorstellungen einer breiten Öffentlichkeit verständlich zu machen. Dieser Erfolg wurde den Protagonisten der Ide-

1 Vgl. Claus Noé, Die Ordnung der Wölfe, in: DIE ZEIT, Nr.11, 10. März 1995, S. 30. 
en der Supply-Side Policy dadurch erleichtert, daß das Kernstück der Theorie die Forderung nach massiven Steuersenkungen war. Mit Steuersenkungen, die sogar noch die wirtschaftlichen Probleme lösen sollen, ließen sich die Wähler und damit die Politiker, die die angebotsorientierte Wirtschaftspolitik umsetzen sollten, ködern.

Die Laffer-Kurve war ein geeignetes, weil einfaches, Instrumentarium, um aufzuzeigen, wie dies funktionieren sollte. Nach der empirischen Relevanz der Kurve hat in den U.S.A. kaum jemand gefragt. Wer weniger Steuern zu zahlen hat, leistet mehr, dieser Sachverhalt erschien einleuchtend.

Das dritte Kapitel machte deutlich, daß Ronald Reagan mit seinem Programm die Konzeption der Supply-Side Policy aufgegriffen hat. Mit dem Wechsel der Präsidentschaft im Jahr 1988 von Reagan zu Bush wurde kein grundsätzlicher Wechsel der Grundausrichtung der Politik der Reaganomics in den Vereinigten Staaten vollzogen. Die Projektion, die Reagan in seinem Programm für die Entwicklung der wirtschaftlichen Referenzgrößen zugrundelegte, machte deutlich, die Laffer-Kurve war Bestandteil der Reaganomics, auch wenn dies im nachhinein von manchen bestritten wurde. Die Reagan-Politik hat maßgeblich darauf gesetzt, da $B$ die Steuersenkungen einen Impuls für die Angebotsseite darstellen und in Folge ein verstärktes Wirtschaftswachstum auslösen, was letztlich zu wachsenden Steuereinnahmen führen sollte; der Laffer-Effekt par excellence.

Das Reagan-Wirtschaftsprogramm aus dem Jahr 1981 suggerierte, daß die angebotsorientierte Wirtschaftspolitik spätestens nach 5 Jahren ihre volle Wirkung entfaltet habe. Es kam leider anders. Das starke Wirtschaftswachstum, hohe Investitionen und der Budgetüberschuß blieben aus.

Dies hing damit zusammen, daß die Steuersenkungen zum Teil wieder rückgängig gemacht werden mußten, weil sich die Konjunktur zu Beginn der ersten Amtszeit Reagans in einer rezessiven Phase befand und dadurch die Steuereinnahmen stärker zurückgingen als erwartet. Hinzu kam, daß die Staatsausgaben, die eigentlich gesenkt werden sollten, aufgrund steigender Soziallasten und Verteidigungsausgaben stark anstiegen. Die jährlichen Budgetdefizite des Bundeshaushaltes erreichten aufgrund dieser Entwicklung in den 80er Jahren in den Vereinigten Staaten Höchststände.

Die konjunkturelle Situation und die schwierige Haushaltslage wurde außerdem durch eine restriktive Geldpolitik der amerikanischen Zentralbank belastet. Aus dieser Geldpolitik resultierte zunächst ein stark steigender Außenwert des US-Dollars, was dazu führte, daß es zu immensen Handelsbilanzdefiziten kam. Im Zusammenhang mit dem Haushaltsdefizit sprach man nunmehr von einem Zwillingsdefizit. Die wirtschaftliche Erholung setzte erst ein, als die amerikanische Zentralbank Mitte der 80er Jahre nach und nach die geldpolitischen Zügel lockerte.

Die langfristige ökonomische Analyse der Reaganomics zeigte anhand der Entwicklung wichtiger Wirtschaftsindikatoren, da $\beta$ die Ergebnisse der angebotsorientierten Wirtschaftspolitik im Vergleich zu Zeiträumen seit den 60er Jahren bescheiden ausgefallen sind. Wirtschaftswachstum, Investitionen und das Sparaufkommen der Jahre 1982 bis 1992 waren im Durchschnitt niedriger als in den Vegleichsperioden. Der 
Staatssektor, der eigentlich kleiner werden sollte, nahm in Anteilen am Bruttoinlandsprodukt sogar zu. Der US-Dollar war starken Wechselkursschwankungen ausgesetzt, zum Ende der Reaganomics hatte der Dollar erheblich an Außenwert verloren.

Die U.S.A. konvertierte in den 80erJahren vom einstmals größten Gläubiger zur größten Schuldnernation.

Der Lebensstandard gemessen an der Einkommensentwicklung stagnierte, für die niedrigeren Einkommensbezieher war er sogar rückläufig. Die Einkommensverteilung verschob sich zugunsten der hohen Einkommen. Die Zahl der Menschen, die in den Vereinigten Staaten unter der Armutsgrenze leben, erhöhte sich von 1981 bis 1992 um 5 Millionen.

Positiv war anzumerken, daß im internationalen Vergleich die Reaganomics hinsichtlich der Entwicklung des Wirtschaftswachstums relativ gut abgeschnitten hat.

Gemessen jedoch am ursprünglichen Anspruch der Supply-Side Policy und den wirtschaftlichen Ergebnissen der Reaganomics mußte jedoch zum Abschluß des 3. Kapitels festgestellt werden, daß die angebotsorientierte Wirtschaftspolitik gescheitert ist.

Das 4. Kapitel ging der Frage nach, ob das Scheitern der Reaganomics dadurch zu erklären ist, daß die marginale Steuerquote - die zentrale Referenzgröße der Angebotstheorie für die wirtschaftliche Entwicklung - in den U.S.A. nicht gesenkt werden konnte.

Hierzu wurde die marginale Steuerquote für die Gebietskörperschaften und unterschiedliche Zeiträume geschätzt. In der Tat ergab sich aus den Ergebnissen der Schätzung, daß der Grenzsteuersatz in den Jahren von 1982 bis 1992 für die gesamte U.S.A. nur unmerklich niedriger war als in der Vorperiode.

Die Analyse der Schätzergebnisse, differenziert nach den einzelnen Gebietskörperschaften, lieferte die Antwort darauf, warum sich der Grenzsteuersatz trotz Steuersenkungen der Reaganomics nicht veränderte. Der Bund hatte zwar einen signifikanten Rückgang der marginalen Steurquote erreicht, aber diese Entwicklung wurde konterkarriert durch die steigende marginale Steuerquote auf der Ebene der Bundesstaaten und Gemeinden.

Die Finanzpolitik des Bundes, die in der Zeit der Reaganomics geringere Finanzzuweisungen an die Bundesstaaten und Gemeinden vorsah, führte letztendlich dazu, daß der marginale Steuersatz nicht gesenkt werden konnte.

Am Ende des 4. Kapitels wird auf der Grundlage der Schätzergebnisse in Frage gestellt, ob selbst bei einer Senkung des Grenzsteuersatzes die erhoffte Wirkung erzielt worden wäre.

Die vorliegende Ausarbeitung läßt zwei Interpretationen der Supply-Side Policy zu:

1. Das Theoriegebäude der Supply-Side Policy hat Schwächen, was die Einschätzung der Anreizwirkungen der Steuern auf das Verhalten der Wirtschaft insgesamt und jedes einzelnen Wirtschaftssubjektes betrifft.

2. Die Laffer-Kurve und der daraus resultierende Wirkungszusammenhang sind ein seltener Spezialfall. Es lassen sich kaum Referenzbeispiele finden, die eine derart hohe marginale Steuerquote aufweisen, so daß mit Sicherheit gesagt werden kann, 
daß man sich im prohibitiven Bereich der Besteuerung befindet. ${ }^{2}$ Für die Vereinigten Staaten kann mit großer Sicherheit gesagt werden, daß sie sich in der Zeit der Reaganomics nicht im prohibitiven Bereich der Laffer-Kuve bewegt hat.

Die Supply-Side Policy und der Versuch ihrer Umsetzung können hinsichtlich ihrer Wirkung auf die amerikanische Wirtschaft nur schwer abgeschätzt werden. Ein positiver Ansatz für eine abschließende Einschätzung wäre wohl der, daß man aufgrund der Ergebnisse, die die Schätzung der marginalen Steuerquote brachte, davon ausgehen würde, daß die Supply-Side Policy gar nicht funktionieren konnte. Dies lag an dem Umstand, daß es im Rahmen der Reaganomics nicht gelang, die Grenzsteuersätze zu senken. Ein Test der Supply-Side Policy, der damit keiner war.

Ein negativer Ansatz zur Beurteilung wäre, daß die Ergebnisse der Reaganomics gezeigt haben, daß die Supply-Side Policy überhaupt nicht funktionieren kann. Der Mensch, die Politiker und die Wirtschaft handeln nach anderen Kriterien, als die Supply-Side Policy glaubhaft machen möchte.

Welcher der aufgezeigten Ansätze hat nun Gültigkeit?

Es spricht viel dafür, daß eher der negative Ansatz zutrifft. Der positive Ansatz läßt unberücksichtigt, daß die Steuern auf Bundesebene tatsächlich erheblich gesenkt wurden. Zudem ignoriert er die Frage, ob eine Reduzierung der Steuerbelastung wirklich das alleinige wirtschaftliche Instrumentarium ist, das über Wohl oder Wehe einer Volkswirtschaft entscheidet. Die Supply-Side Policy war zu trivial angelegt. Sie vergaß in ihrer Konzeption weitere wirtschaftliche Parameter einzubeziehen.

Der negative Ansatz wäre jedoch ebenfalls zu einseitig angelegt für eine abschlägige Bewertung der angebotsorientierten Wirtschaftspolitik. Ohne Zweifel werden über die Steuerpolitik Anreize für das wirtschaftliche Handeln gesetzt, dies hat die Supply-Side Policy wiedererkannt. Das aktuelle Aufleben der Supply-Side Policy in den Vereinigten Staaten zeigt, daß die Erfahrungen mit der Reaganomics nicht zu einer generellen Ablehnung der angebotsorientierten Wirtschaftspolitik geführt haben. Die Ergebnisse der Reaganomics drängen aus makroökonomischer Sicht die Überlegung auf, daß die Wirtschaftspolitik sich nicht ausschließlich mit ihren Maßnahmen auf eine Seite der Wirtschaft ausrichten sollte. Akzeptiert man diese Hypothese, ergibt sich daraus die Konsequenz der Forderung nach einem Policy-Mix aus einer angebots- und nachfrageorientierten Wirtschaftspolitik. Diese Forderung ist keineswegs paradox. Sowohl die nachfrage- als auch die angebotsorientierte Wirtschaftspolitik können sich sinnvoll ergänzen. $^{3}$

Gerade im Hinblick auf die Reaganomics ist die Trennung zwischen angebots- und nachfrageorientierter Wirtschaftspolitik nicht einfach. Könnte der wirtschaftliche Erholungsprozeß, der in den U.S.A. Mitte der 80er Jahre einsetzte, nicht ein Ergebnis der

2 Vgl. Stiglitz, J. E., Finanzwissenschaft, München 1989, S. 136.

3 Vgl. Oberhauser, A., Geknebelte Beschäftigungspolitik - eine Folge der Maastricht-Kriterien, in: Bolte, K. M., u.a. (Hrsg.), Mitteilungen aus der Arbeitsmarkt- und Berufsforschung, 2/96, S. 228236. 
enormen Ausweitung des Staatsdefizits, das wie ein staatliches Konjunkturprogramm gewirkt haben könnte, gewesen sein?4

Die Erfahrungen der nachfrage- und angebotsorientierten Wirtschaftspolitik haben gezeigt, daß insbesondere wirtschaftliche Krisensituationen einer Koordination zwischen Finanz- und Geldpolitik bedürfen. Die Finanzpolitik muß dabei die Wirkungsrichtung der Geldpolitik berücksichtigen. Die Zentralbank hat, dies läßt sich mittlerweile verallgemeinern, die rudimentäre Aufgabe, den Geldwert zu stabilisieren. Auf dieser Stabilitätsgrundlage kann die Finanzpolitik in Zusammenarbeit mit der Notenbank ein geordnetes Schuldenmanagement betreiben. ${ }^{5}$

Ein sinnvoller Policy-Mix bedeutet,

„daß die Träger der Wirtschaftspolitk die Notwendigkeit einer koordinierten Makropolitik begreifen und in der Durchsetzung gesamtwirtschaftlicher Ziele zusammenarbeiten. "6

In den Koordinierungsprozeß für die Gestaltung der Wirtschaftspolitik müssen die Hauptakteure eingebunden werden. Dazu gehören alle Ebenen der Finanzpolitik, die Zentralbank, die Unternehmer und die Gewerkschaften.

Bei allem Theoriestreit, der zwischen den Denkschulen der Wirtschaftswissenschaften mittlerweile seit Jahrzehnten ausgetragen wird, bleibt bisweilen vergessen, daß nachfrage- und angebotsorientierte Wirtschaftspolitik sich nicht gegenseitig aussschlieBen. Sie können sich durchaus sinnvoll ergänzen, bedürfen aber der Bereitschaft zur Koordination. Diese Bereitschaft, das zeigt die wirtschaftspolitische Praxis, ist nicht sehr ausgeprägt.

Allen Wirtschaftspolitikern, Unternehmern, Gewerkschaftern und Privatpersonen, die aktuell massive Steuersenkungen fordern und den Ruf nach weniger staatlichen Einfluß erheben, sollte das Beispiel der Reaganomics mehr als nur zu denken geben.

4 Vgl. Krupp, H.-J., Programm ohne Erfolg, in: DIE ZEIT, Nr. 12, 18. März 1994, S. 24 und Oberhauser, A., Geknebelte Beschäftigungspolitik - eine Folge der Maastricht-Kriterien, in: Bolte, K. M., u.a. (Hrsg.), Mitteilungen aus der Arbeitsmarkt- und Berufsforschung, 2/96, S. 235.

5 Vgl. Ketterer, K.-H., Staatsverschuldung und Geldpolitik, in:Simmmert, D. B., und Wagner, K.-D. (Hrsg.), Staatsverschuldung kontrovers, Schriftenreihe der Bundeszentrale für politische Bildung. Bonn 1981, S. 328-345.

6 Vgl. Krupp, H.-J., Die Koordination von Geld-, Finanz- und Einkommenspolitik als Augabe der Wirtschaftspolitik, in: Wirtschaftsdienst 1994/IV, S. 216. 
Joachim Nagel - 978-3-631-75238-8

Downloaded from PubFactory at 01/11/2019 06:47:33AM

via free access 


\section{Literaturverzeichnis}

Akhtar, M.A., Harris, E.S., The Supply-Side Consequence of U.S. Fiscal Policy in the 1980s, in: Federal Reserve Bank of New York, Quarterly Review, Vol. 17, Nr.1, 1992.

Barro, R. J., Sahasakul, C., Measuring the Average Marginal Tax Rate from the Individual Income Tax, Journal of Business, vol. 56, no. 4, 1983, S. 419-452.

Barth, J. R., The Reagan Program for economic recovery: Economic Rationale; Federal Reserve Bank of Atlanta, Economic Review, September 1981, S. 4-14.

Bartlett, B. R., Reaganomics: supply-side economics in action, Westport 1981.

Bartley, R. L., The seven fat years-and how to do it again, New York 1995.

Baumol, W. J., Blinder, A. S., Economics, Principles and Policy, sixth edition, 1994.

Baumol, W. J., Say's (at least) Eight Laws, or What Say and James Mill may really have meant, in: Economica, 1977, Vol. 44, S. 145-161.

Bishop, J., „Is the Test Score Decline Responsible for the Productivity Growth Decline?", American Economic Review, vol. 79, März 1989, S. 178-197.

Blinder, A. S., Comments on „Why is U.S. National Saving So Low ?“", Brookings Papers on Economic Activity, 2, Summers, L. und Carrol, C. (Hrsg.), 1987.

Blinder, A. S., Thoughts on the Laffer Curve, in Meyer, L. A. (Hrsg.), The Supply-side Effects of Economic Policy, Boston 1981, S. 81-92.

Boskin, M., Reagan and the Economy, The Success, Failures and Unfinished Agenda, San Francisco (CA) 1987.

Boskin, M., Taxation, Saving , and the Rate of Interest, in: Journal of Political Economics, 86, 1978, S. 3-27.

Brown, C., Taxation and the incentive to work, Oxford 1983.

DIE ZEIT, Angriff auf die Armen, Nr. 21, vom 19. Mai 1995, S. 21-22.

DIE ZEIT, Das Ende der Mittelklasse, Nr. 18, vom 26. April 1996, S. 20.

Dupuit, J., On the measurement of the utility of public works, 1844.

Eckstein, O., A Time for Supply Economics, in: Fink, R. H. (Hrsg.), Supply-Side Economics: A Critical Appraisal, Maryland 1982, S. 231-244.

Economic Report of the President, Februar 1982.

Economic Report of the President, Februar 1994.

Ehring, D., Keynes, die Globalsteuerung und die Stabilisierungspolitik in der Bundesrepublik Deutschland, Frankfurt am Main 1989.

Federal Reserve Bank of Atlanta (Hrsg.), Suppy-Side Economics in the 1980s, Atlanta 1982.

Federal Reserve Bank of Atlanta, Economic Review, September 1981. 
Feige, E. L., McGee, R. T., Sweden`s Laffer Curve: Taxation and the Unobserved Economy, Scandinavian Journal of Economics 85 (4), 1983, S. 499-519.

Felderer, B., Homburg, S., Makroökonomik und Neue Makroökonomik, Berlin 1987.

Feldstein, M., American Economic Policy in the 1980s: A personal view, in Feldstein, M. (Hrsg.), American Economic Policy in the 1980s, Chicago 1994, S. 1-79.

Filc, W., Monetäre Fundierung einer angebotsorientierten Stabilitätspolitik, in: "Wirtschaftspolitische Diskurse" Reihe 37 des Forschungsinstitut der Friedrich-EbertStiftung, 25 Jahre Stabilitätsgesetz: Überlegungen zu einer zeitgerechten Ausgestaltung der Stabilitäts- und Wachstumspolitik, S. 25-S. 49.

Fink, R. H. (Hrsg.), Supply-Side Economics: A Critical Appraisal, Maryland 1982.

Fink, R. H., Introduction, in: Fink, R. H. (Hrsg.), Suppy-Side Economics: A Critical Appraisal, Maryland 1982, S. xiiv-xxiv.

Flassbeck, H., Was ist Angebotspolitik ?, Konjunkturpolitik, Jg. 28 (1982), Heft 2/3, S. 75-138.

Frankel, J. A., Exchange Rate Policy, in Feldstein, M. (Hrsg.), American Economic Policy in the 1980s, Chicago 1994, S. 293-341.

Fullerton, D., On the possibility of an inverse relationship between tax rates and government revenues, in: Journal of Public Economics 19, 1982, S. 3-22.

Fullerton, D., Tax Policy, in: Feldstein, M. (Hrsg.), American Economic Policy in the 1980s, Chicago 1994, S. 165-208.

Galbraith, J. K., Money, Whence it came, where it went, Boston - New York 1995.

Gilder, G., Reichtum und Armut, Berlin 1981.

Gilder, G., The Supply-Side, in: Fink, R. H. (Hrsg.), Supply-Side Economics: A Critical Appraisal, Maryland 1982, S. 29-31.

Gilder, G., Wealth and Poverty, New York 1981.

Hailstones, T. J., A Guide to Supply-side Economics, Richmond 1982.

Hansemeyer, K. H., Lehr- und Methodengeschichte, in: Kompendium der Volkswirtschaftslehre, Band 1, Göttingen 1967, S. 485-486.

Henderson, D., Limitations of the Laffer Curve as a Justification for Tax Cuts,

in: Fink, R. (Hrsg.): Supply-Side Economics: A Critical Appraisal, Frederick, Maryland 1982, S. 224-230.

Hirschman, A. O., Interests, in: The New Palgrave: The World of Economics, in: Economic Impact, No. 35, 1981/3, S. 349-370.

Holtfrerich, C.-L., Wirtschaft USA, München 1991.

Horst, P., Haushaltspolitik und Regierungspraxis in den USA und der Bundesrepublik Deutschland: ein Vergleich des haushaltspolitischen Entscheidungsprozesses beider Bundesrepubliken zu Zeiten der konservativen Regierung Reagan/Bush (1981 - 92) und Kohl (1982 - 93), Frankfurt am Main 1995.

Issing, O., Angebotsorientierte Wirtschaftspolitik, in: WiSt, Heft 10, Oktober 1982, S. 463-468.

Issing, O., Die Laffer-Kurve, in: WiSt, Heft 8, August 1981, S. 384-386. 
Issing, O., Supply-Side Economics-Marginalien zu einem wirtschaftspolitischem Programm, in: Woll, A. (Hrsg.): Aktuelle Probleme der Wirtschaftspolitik, Schriften des Vereins für Socialpolitik, NF Bd. 130, Berlin 1983, S.139-156.

Jetha, N., Taxation and economic behaviour, Bulletin for International Fiscal Documentation, 34, 1980, S. 156-160.

Joint Economic Committee, Joint Economic Report, Senate Report No. 96-44, 96th Cong., 2d sess., 1980.

Keleher, R. E., Evidence Relating to Supply-Side Tax Policy, in: Fink, R. H. (Hrsg.), Supply-Side Economics: A Critical Appraisal, Maryland 1982, S. 264-275.

Keleher, R. E., Orzechowski, W. P., Supply-Side Fiscal Policy: An Historical Analysis of a Rejuvenated Idea, in: Fink, R. H. (Hrsg.), Supply-Side Economics: A Critical Appraisal, Maryland 1982, S. 121-159.

Keller, K. J., Wirtschaftspolitik in den USA, Das wirtschaftspolitische Programm der Regierung Reagan und seine Hintergründe, in: WiSt Heft 5, Mai 1982, S. 230-232.

Kemp, J., Supply-Side Economics: An American Renaissance, in: Suppy-Side Economics in the 1980s, Federal Reserve Bank of Atlanta 1982, S. 27-39.

Ketterer, K.-H., Staatsverschuldung und Geldpolitik, in: Simmmert, D. B., und Wagner, K.-D. (Hrsg.), Staatsverschuldung kontrovers, Schriftenreihe der Bundeszentrale für politische Bildung, Bonn 1981, S. 328-345.

Keynes, J. M., Allgemeine Theorie der Beschäftigung, des Zinses und Geldes, Berlin 1983.

Keynes, J. M., The General Theory of Employment, Interest and Money,London 1936.

Kneer, J., Reaganomics-Elemente der amerikanischen Wirtschaftspolitik, in: Wirtschaftsdienst 1982/I, S. 28-34.

Koch, W. A. S., Erhöhen Steuersenkungen die Leistungsbereitschaft?, in: Wirtschaftsdienst 65, 1985, S. 531-536.

Koester, R. B., Kormendi, R. C., Taxation, Aggregate Activity and Economic Growth: Cross-Country Evidence on some Supply-Side Hypothesis, Economic Inquiry 27, S. 367-386.

Kristol, I., Ideology and Supply-side Economics, in: Commentary, Economic Inquiry, 71, April 1981, S. 48-54.

Kristol, I., Toward a 'New' Economics?, Wall Street Journal, 9. Mai 1977.

Krugman, P., Peddling Prosperity: economic sense and nonsense in the age of diminished expectations, New York-London 1994.

Krupp, H.-J., Die Koordination von Geld-, Finanz- und Einkommenspolitik als Augabe der Wirtschaftspolitik, in: Wirtschaftsdienst 1994/IV, S. 208-216.

Krupp, H.-J., Programm ohne Erfolg, in: DIE ZEIT, Nr. 12, 18. März 1994, S. 24.

Kurz, R., Angebotsorientierte Wirtschaftspolitik in den USA: Grundlagen, Praxis und Konsequenzen, Tübingen 1993.

Laffer, A. B., An Equilibrium Rational Macroeconomics Framework, in: Kamrany, N. M., Day, R. H. (Hrsg.), Economic Issues of the Eighties, Baltimore 1979, S. 44-57. 
Laffer, A. B., Statement prepared for the Joint Economic Committee, in: The economicsof the tax revolt: A reader, Laffer, A. B. und Seymour, J. P. (Hrsg.), New York 1979.

Laffer, A. B., Supply-Side Economics, in: Financial Analysts Journal, 37, 1981, S. 2943.

Laffer, A. B., W. P., Government Exactions and Revenue Deficiencies, in: Fink, R. H. (Hrsg.), Supply-Side Economics: A Critical Appraisal, Maryland 1982, S. 185-203.

Lamping, H., Supply-Side Economics, Oldenburg 1988.

Landmann, O., Keynes in der heutigen Wirtschaftstheorie, in: Bombach et al. (Hrsg.): Keynesianismus I, Berlin-Heidelberg-New York 1981, S. 133-210.

Leber, M., „Reaganomics“ als wirtschaftspolitisches Vermächtnis, Dokumentation zur Wirtschaftskunde, Nr. 12, Dezember 1989, S. 3-9.

Los Angeles Times, vom 13. August 1996, S. A17.

Malabre, A. L., Ungehörte Propheten: eine Insider Geschichte der modernen Ökonomie, Stuttgart 1994.

Moore, T. G., Die Wirtschaftspolitik der Reagan-Administration, in: Vogel, O. (Hrsg.), Wirtschaftspolitik der achtziger Jahre, Köln 1982, S. 277-312.

Moszer,M., A Comment on the Laffer Model, in: Fink, R. H. (Hrsg.), Supply-Side Economics: A Critical Appraisal; Maryland 1982, S. 204-223.

Müller-Oestreich, K., Der Einfluß der Finanzpolitik auf die Investitionsentwicklung in den USA unter Ronald Reagan, Berlin 1992.

Mundell, R. A., The Dollar and the Policy Mix: 1971, Essays in International Finance, International Finance Section, no. 85, May, Princeton 1981, S. 3-28.

Mundell, R. A., The Dollar and the Policy Mix: 1971, in: Recent Developments in Macroeconomics, Phelps, E. S. (Hrsg.), S. 390-415.

National Income and Product Accounts of the United States, Volume 2, 1959 88, U.S. Department of Commerce, New York 1991.

Noé, C., Die Ordnung der Wölfe, in: DIE ZEIT, Nr.11, 10. März 1995, S. 30.

Oberhauser, A., Geknebelte Beschäftigungspolitik - eine Folge der Maastricht-Kriterien, in: Bolte, K. M., u.a. (Hrsg.), Mitteilungen aus der Arbeitsmarkt- und Berufsforschung, 2/96, S. 228-236.

Palmer, J. L.,Sawhill, I. V., The Reagan Experiment: An Examination of Economic and Social Policies under the Reagan Administration, Washington, D.C. 1982.

Rahmann, B., Ist Angebotsökonomik eine instrumentelle Alternative zur keynesianischen Nachfrageökonomik?, in: Buttler, F., Kühl, J., Rahmann, B., Hrsg., Staat und Beschäftigung, Angebots- und Nachfragepolitik in Theorie und Praxis, Beiträge zur Arbeitsmarkt- und Berufsforschung, Institut für Arbeitsmarkt und Berufsforschung der Bundesanstalt für Arbeit, Nürnberg 1985., S. 65-100.

Reagan, R., First Inaugural Address, 20. Januar 1981, in: http://www.columbia.edu/acis/bartleby/inaugural/pres61.html, vom 12.09.1996.

Reich, H., Eigennutz und Kapitalismus: die Bedeutung des Gewinnstrebens im klassischen ökonomischen Denken, Berlin 1991. 
Richardson, J. D., Trade Policy, in Feldstein, M. (Hrsg.), American Economic Policy in the 1980s, Chicago 1994, S. 627-658.

Roberts, P. C., Angebotsorientierte Wirtschaftspolitik, theoretische Grundlagen und politische Erfolge: Eine Bewertung der amerikanischen Erfahrungen in den achtziger Jahren, Zeitschrift für Wirtschaftspolitik, Heft 1, 1989, S. 5-42.

Roberts, P. C., Supply-Side Economics: An Assessment of The American Experience In The 1980s, Februar 1989, National Westminster Bank Quarterly Review, S. 6074.

Roberts, P. C., The Breakdown of the Keynesian Model, in: Fink, R. H. (Hrsg.), Suppy- Side Economics: A Critical Appraisal, Frederick, Maryland 1982, S. 1-13.

Salant, W. S., A Critical Look at Supply-Side Theory and a Brief Look at some of its International Aspects, in: International Monetary Problems and Supply-Side Economics, Cohen, J. S. and Harcourt, G. C. (Hrsg.), London 1986, S. 108-124.

Say, J. B., Traite d'economie politique, Paris 1803.

Schaal, P., Reaganomics - Konzept und Perspektiven der neuen amerikanischen Wirtschaftspolitik, List-Forum, Band 11 (1982), S. 391-411.

Schultze, C. L., Memos to the president: a guide through macroeconomics for the busy policymaker, The Brookings Institution, Washington, D. C. 1992.

Schumpeter, J. A., Geschichte der ökonomischen Analyse, Göttingen 1965.

Stiglitz, J. E., Finanzwissenschaft, München 1989.

Stockman, D., Der Triumph der Politik, Die Krise der Reagan-Regierung und ihre Auswirkungen auf die Weltwirtschaft, München 1986.

Survey of Current Business, U.S. Department of Commerce, July 1992, Volume 72 , Number 7.

Survey of Current Business, U.S. Department of Commerce, March 1993, Volume 73, Number 3.

The Wall Street Journal, New Economics, Leitartikel vom 8. Oktober 1981.

The Wall Street Journal, Review and Outlook, Leitartikel vom 4. August 1976.

The Wall Street Journal, Tax-Cut Theorists, Leitartikel vom 1. Dezember 1978.

The White House, Office of the Press Secretary: A Program for Economic Recovery, Washington D.C., February 18, 1981.

The White House, Office of the Press Secretary: President Reagan`s Initiatives to Reduce Regulatory Burdens, Washington D.C., February 18, 1981, S. 1.

Tobin, J., Policies for Prosperity: Essays in a Keynesian Mode, Economic Stabilization Policies in the United States, Brighton 1987, S. 439-453.

Tobin, J., Policies for Prosperity: Essays in a Keynesian Mode, Monetarism: An Ebbing Tide?, Brighton 1987, S. 265-274.

Tobin, J., Policies for Prosperity: Essays in a Keynesian Mode, Monetary Policy in an Uncertain World, Brighton 1987, S. 240-254.

Tobin, J., Policies for Prosperity: Essays in a Keynesian Mode, The Political Economy of the 1960s, Brighton 1987, S. 422-438. 
Tobin, J., Policies for Prosperity: Essays in a Keynesian Mode, Yes, Virginia, There Are Laffer Curves, Brighton 1987, S. 120-125.

Tobin, J., The Monetary and Fiscal Policy Mix, in: Recent Developments in Macroeconomics, Phelps, E. S. (Hrsg.), 1991, S. 508-520.

U.S. Department of Commerce, Economics and Statistics Administration Bureau of Economic Analysis, National Income and Product Accounts of the United States, Volume 2, 1959-88, New York 1991.

von Fürstenberg, G., Saving, in: Aaron, A., Pechman, J (Hrsg.), How taxes affect economic behaviour, Washington 1981, S. 327-402.

Wachtel, P., Makroökonomik: von der Theorie zur Praxis, Oldenburg 1994.

Weidenbaum, M., Regulatory Reform: A Fast Start, in: Economic Impact, 1981/4, S. $8-10$.

Wendisch, P., Supply-Side Policy, Theoretische Grundlagen und prozeßpolitische Konsistenz, Frankfurt am Main 1984.

Winkler, A., Geld, Zins und keynesianische Angebotspolitik, Berlin 1992.

Wittmann, F., Die Berücksichtigung von Steuern bei Investitionsentscheidungen der Unternehmen, WSI-Mitteilungen, 39, 1986, S. 782-790.

Zschiegener H., Habert U., Einzelfragen der Übergangsregelungen der US- Steuerreform 1986, in: Internationale Wirtschaftsbriefe (IWB), Nr.12 vom 25.6. 1987, S. 391-398. 


\section{FINANZWISSENSCHAFTLICHE SCHRIFTEN}

Band 1 Werner Steden: Finanzpolitik und Einkommensverteilung. Ein Wachstums- und Konjunkturmodell der Bundesrepublik Deutschland. 1979.

Band 2 Rainer Hagemann: Kommunale Finanzplanung im föderativen Staat. 1976.

Band 3 Klaus Scherer: Maßstábe zur Beurteilung von konjunkturellen Wirkungen des öffentlichen Haushalts. 1977.

Band 4 Brita Steinbach: "Formula Flexibility" - Kritische Analyse und Vergleich mit diskretionärer Konjunkturpolitik. 1977.

Band 5 Hans-Georg Petersen: Personelle Einkommensbesteuerung und Inflation. Eine theoretisch-empirische Analyse der Lohn- und veranlagten Einkommensteuer in der Bundesrepublik Deutschland. 1977.

Band 6 Friedemann Tetsch: Raumwirkungen des Finanzsystems der Bundesrepublik Deutschland. Eine Untersuchung der Auswirkungen der Finanzreform von 1969 auf die Einnahmenposition der untergeordneten Gebietskörperschaften und ihrer regionalpolitischen Zieladăquanz. 1978.

Band 7 Wilhelm Pfähler: Normative Theorie der fiskalischen Besteuerung. Ein methodologischer und theoretischer Beitrag zur Integration der normativen Besteuerungstheorie in der Wohlfahrtstheorie. 1978.

Band 8 Wolfgang Wiegard: Optimale Schattenpreise und Produktionsprogramme für öffentliche Unternehmen. Second-Best Modelle im finanzwirtschaftlichen Staatsbereich. 1978.

Band 9 Hans P. Fischer: Die Finanzierung des Umweltschutzes im Rahmen einer rationalen Umweltpolitik. 1978.

Band 10 Rainer Paulenz: Der Einsatz finanzpolitischer Instrumente in der Forschungs- und Entwicklungspolitik. 1978.

Band 11 Hans-Joachim Hauser: Verteilungswirkungen der Staatsverschuldung. Eine kreislauftheoretische Inzidenzbetrachtung. 1979.

Band 12 Gunnar Schwarting: Kommunale Investitionen. Theoretische und empirische Untersuchungen der Bestimmungsgründe kommunaler Investitionstătigkeit in NordrheinWestfalen 1965-1972. 1979.

Band 13 Hans-Joachim Conrad: Stadt-Umland-Wanderung und Finanzwirtschaft der Kernstädte. Amerikanische Erfahrungen, grundsătzliche Zusammenhänge und eine Fallstudie für das Ballungsgebiet Frankfurt am Main. 1980.

Band 14 Cay Folkers: Vermōgensverteilung und staatliche Aktivităt. Zur Theorie distributiver Prozesse im Interventionsstaat. 1981.

Band 15 Helmut Fischer: US-amerikanische Exportförderung durch die DISC-Gesetzgebung. 1981.

Band 16 Günter Ott: Einkommensumverteilungen in der gesetzlichen Krankenversicherung. Eine quantitative Analyse. 1981.

Band 17 Johann Hermann von Oehsen: Optimale Besteuerung. (Optimal Taxation). 1982.

Band 18 Richard Kōssler. Sozialversichenungsprinzip und Staatszuschüsse in der gesetzlichen Rentenversicherung. 1982.

Band 19 Hinrich Steffen: Zum Handlungs- und Entscheidungsspielraum der kommunalen Investitionspolitik in der Bundesrepublik Deutschland. 1983.

Band 20 Manfred Scheuer: Wirkungen einer Auslandsverschuldung des Staates bei flexiblen Wechselkursen. 1983. 
Band 21 Christian Schiller: Staatsausgaben und crowding-out-Effekte. Zur Effizienz einer Finanzpolitik keynesianischer Provenienz. 1983.

Band 22 Hannelore Weck: Schattenwirtschaft: Eine Möglichkeit zur Einschränkung der öffentlichen Verwaltung? Eine ökonomische Analyse. 1983.

Band 23 Wolfgang Schmitt: Steuern als Mittel der Einkommenspolitik. Eine Ergänzung der Stabilitätspolitik? 1984.

Band 24 Wolfgang Laux: Erhöhung staatswirtschaftlicher Effizienz durch budgetäre Selbstbeschränkung? Zur ldee einer verfassungsmäßig verankerten Ausgabengrenze. 1984.

Band 25 Brita Steinbach-van der Veen: Steuerinzidenz. Methodologische Grundlagen und empirisch-statistische Probleme von Länderstudien. 1985.

Band 26 Albert Peters: Ökonomische Kriterien für eine Aufgabenverteilung in der Marktwirtschaft. Eine deskriptive und normative Betrachtung für den Allokationsbereich. 1985.

Band 27 Achim Zeidler: Möglichkeiten zur Fortsetzung der Gemeindefinanzreform. Eine theoretische und empirische Analyse. 1985.

Band 28 Peter Bartsch: Zur Theorie der längerfristigen Wirkungen 'expansiver' Fiskalpolitik. Eine dynamische Analyse unter besonderer Berücksichtigung der staatlichen Budgetbeschränkung und ausgewählter Möglichkeiten der öffentlichen Defizitfinanzierung. 1986.

Band 29 Konrad Beiwinkel: Wehrgerechtigkeit als finanzpolitisches Verteilungsproblem. Möglichkeiten einer Kompensation von Wehrungerechtigkeit durch monetäre Transfers. 1986.

Band 30 Wolfgang Kitterer: Effizienz- und Verteilungswirkungen des Steuersystems. 1986.

Band 31 Heinz Dieter Hessler: Theorie und Politik der Personalsteuern. Eine Kritik ihrer Einkommens- und Vermögensbegriffe mit Blick auf die Leistungsfähigkeitstheorie. 1994.

Band 32 Wolfgang Schert: Die beschäftigungspolitische und fiskalische Problematik der Arbeitgeberbeiträge zur Rentenversicherung. Eine Auseinandersetzung mit der Kritik an der lohnbezogenen Beitragsbemessung. 1987.

Band 33 Andreas Mästle: Die Steuerunion. Probleme der Harmonisierung spezifischer Gütersteuern. 1987.

Band 34 Günter Ott: Internationale Verteilungswirkungen im Finanzausgleich der Europäischen Gemeinschaften. 1987.

Band 35 Heinz Haller: Zur Frage der zweckmäßigen Gestalt gemeindlicher Steuern. Ein Diskussionsbeitrag zur Gemeindesteuerreform. 1987.

Band 36 Thomas Kuhn: Schlüsselzuweisungen und fiskalische Ungleichheit. Eine theoretische Analyse der Verteilung von Schlüsselzuweisungen an Kommunen. 1988.

Band 37 Walter Hahn: Steuerpolitische Willensbildungsprozesse in der Europäischen Gemeinschaft. Das Beispiel der Umsatzssteuer-Harmonisierung. 1988.

Band 38 Ulrike Hardt: Kommunale Finanzkraft. Die Problematik einer objektiven Bestimmung kommunaler Einnahmemöglichkeiten in der gemeindlichen Haushaltsplanung und im kommunalen Finanzausgleich. 1988.

Band 39 Jochen Michaelis: Optimale Finanzpolitik im Modell überlappender Generationen. 1989.

Band 40 Bernd Raffelhüschen: Anreizwirkungen der sozialen Alterssicherung. Eine dynamische Simulationsanalyse. 1989.

Band 41 Berend Diekmann: Die Anleihe- und Darlehenstransaktionen der Europäischen Gemeinschaften. 1990.

Band 42 Helmut Kaiser: Konsumnachfrage, Arbeitsangebot und optimale Haushaltsbesteuerung. Theoretische Ergebnisse und mikroökonometrische Simulation für die Bundesrepublik Deutschland. 1990. 
Band 43 Rüdiger von Kleist: Das Gramm-Rudman-Hollings-Gesetz. Ein gescheiterter Versuch der Haushaltskonsolidierung. 1991.

Band 44 Rolf Hagedorn: Steuerhinterziehung und Finanzpolitik. Ein theoretischer Beitrag unter besonderer Berücksichtigung der Hinterziehung von Zinserträgen. 1991.

Band 45 Comelia S. Behrens: Intertemporale Verteilungswirkungen in der gesetzlichen Krankenversicherung der Bundesrepublik Deutschland. 1991.

Band 46 Peter Saile: Ein ökonomischer Ansatz der Theorie der intermediären Finanzgewalten Die Kirchen als Parafisci. 1992.

Band 47 Peter Gottfried: Die verdeckten Effizienzwirkungen der Umsatzsteuer. Eine empirische allgemeine Gleichgewichtsanalyse. 1992.

Band 48 Andreas Burger: Umweltorientierte Beschäftigungsprogramme. Eine Effizienzanalyse am Beispiel des "Sondervermögens Arbeit und Umwelt". 1992.

Band 49 Jeanette Malchow: Die Zuordnung verteilungspolitischer Kompetenzen in der Europäischen Gemeinschaft. Eine Untersuchung aufgrund einer Fortentwicklung der ökonomischen Theorie des Föderalismus. 1992.

Band 50 Barbara Seidel: Die Einbindung der Bundesrepublik Deutschland in die Europäischen Gemeinschaften als Problem des Finanzausgleichs. 1992.

Band 51 Ralph Wiechers: Markt und Macht im Rundfunk. Zur Stellung der öffentlich-rechtlichen Rundfunkanstalten im dualen Rundfunksystem der Bundesrepublik Deutschland. 1992.

Band 52 Klaus Eckhardt: Probleme einer Umweltpolitik mit Abgaben. 1993.

Band 53 Oliver Schwarzkopf: Die Problematik unterschiedlicher Körperschaftsteuersysteme innerhalb der EG. 1993.

Band 54 Thorsten Giersch: Bergson-Wohlfahrtsfunktion und normative Ökonomie. 1993.

Band 55 Li-Fang Chou: Selbstbeteiligung bei Arzneimitteln aus ordnungspolitischer Sicht. Das Beispiel der Bundesrepublik Deutschland. 1993.

Band 56 Harald Schlee: Einkommensteuerliche Behandlung von Transferzahlungen. Zur Neuordnung der Familienbesteuerung sowie der Besteuerung von Versicherungsleistungen und Sozialtransfers. 1994.

Band 57 Alexander Spermann: Kommunales Krisenmanagement. Reaktionen baden-württembergischer Stadtkreise auf steigende Sozialhilfekosten und Einnahmenausfälle (198092). 1993.

Band 58 Otto Roloff / Sibylle Brander / Ingo Barens / Claudia Wesselbaum: Direktinvestitionen und internationale Steuerkonkurrenz. 1994.

Band 59 Claudia Wesselbaum-Neugebauer: Internationale Steuerbelastungsvergleiche. 1994.

Band 60 Stephanie Miera: Kommunales Finanzsystem und Bevölkerungsentwicklung. Eine Analyse des kommunalen Finanzsystems vor dem Hintergrund der sich abzeichnenden Bevölkerungsentwicklung am Beispiel Niedersachsens unter besonderer Berücksichtigung des Landkreises Wolfenbüttel und seiner Gemeinden. 1994.

Band 61 Wolfgang Schert: Die Bedeutung des kaldorianischen Verteilungsmechanismus für die gesamtwirtschaftlichen Wirkungen der staatlichen Neuverschuldung. 1994.

Band 62 Rainer Volk: Vergleich der Vergünstigungseffekte der verschiedenen investitionsfördemden Maßnahmen. 1994.

Band 63 Hans-Georg Napp: Kommunale Finanzautonomie und ihre Bedeutung für eine effiziente lokale Finanzwirtschaft. 1994. 2., unveränderte Auflage 1994.

Band 64 Bernd Rahmann / Uwe Steinborn / Günter Vornholz: Empirische Analyse der Autonomie lokaler Finanzwirtschaften in der Europäischen Gemeinschaft. 1994. 
Band 65 Carsten Kühl: Strategien zur Finanzierung der Altlastensanierung. 1994.

Band 66 Stephan Boll: Intergenerationale Umverteilungswirkungen der Fiskalpolitik in der Bundesrepublik Deutschland. Ein Ansatz mit Hilfe des Generational Accounting. 1994.

Band 67 Karl Justus Bernhard Neumärker: Finanzverfassung und Staatsgewalt in der Demokratie. Ein Beitrag zur konstitutionellen Finanztheorie. 1995.

Band 68 Christian Haslbeck: Zentrale versus dezentrale Internalisierung externer Effekte bei unvollständiger Information. 1995.

Band 69 Regina Müller: Horizontale oder vertikale Transfers zur Durchsetzung eines horizontalen Finanzausgleichs. 1995.

Band 70 Christian Hockenjos: Öffentliche Sporttörderung in der Bundesrepublik Deutschland. Darstellung und finanztheoretische Analyse. 1995.

Band 71 Manfred Rosenstock: Die Kontrolle und Harmonisierung nationaler Beihilfen durch die Kommission der Europäischen Gemeinschaften. 1995.

Band 72 Christian Rüsch: Wohnungsbau- und Wohneigentumspolitik im Rahmen der Einkommensteuer. Eine Analyse unter steuersystematischen, verteilungspolitischen und fiskalischen Aspekten. 1996.

Band 73 Stephan Winters: Die kollektive Vorsorge für den Pflegefall im Alter. Eine Untersuchung am Beispiel der gesetzlichen Pflegeversicherung in den Niederlanden. 1996.

Band 74 Knut Blind: Allokationsineffizienzen auf Sicherheitsmärkten: Ursachen und Lösungsmöglichkeiten. Fallstudie: Informationssicherheit in Kommunikationssystemen. 1996.

Band 75 Barbara Petrick-Rump: Ökonomische Wirkungen von Steueramnestien. Untersuchung konkreter Erfahrungen ausgewählter Länder mit dem Einsatz von Steueramnestien anhand eines effizienten Steueramnestieprogramms. 1996.

Band 76 Georg Hirte: Effizienzwirkungen von Finanzausgleichsregelungen. Eine Empirische Allgemeine Gleichgewichtsanalyse für die Bundesrepublik Deutschland. 1996.

Band 77 Ulrike Kirchhoff: Die rheinland-pfälzischen Gemeinden im System des Finanzausgleichs. 1996.

Band 78 Kerstin Keil: Der soziale Mietwohnungsbau: Mängel und Alternativen. 1996.

Band 79 Bernhard Manzke: Kinderlastenausgleich versus verstärkte Einwanderung. Alternative Ansätze zur langfristigen Sicherung der Gesetzlichen Rentenversicherung. 1997.

Band 80 Hariolf M. Wenzler: Institutionenökonomik und öffentliche Finanzkontrolle. Eine Analyse am Beispiel der Europäischen Union. 1997.

Band 81 Joachim Nagel: Supply-Side Policy in den USA. Eine theoretische und empirische Analyse der angebotsorientierten Wirtschaftspolitik Reagans unter besonderer Berücksichtigung steuerlicher Aspekte. 1997. 
Wolfgang Hermann Müller

\section{Prinzipien wirtschaftlichen Handelns und ihre Anwendung}

\section{Umriß einer Wirtschaftsphilosophie}

Frankfurt/M., Berlin, Bern, New York, Paris, Wien, 1996. 145 S., 1 Abb. ISBN 3-631-30477-3 · br. DM 48.-*

Die enge Verbindung von Wirtschaft und Weltgeschehen verlangt nach einer exakten Untersuchung ihrer Notwendigkeit, Möglichkeiten und Grenzen. Die Ökonomie wird auf ihre erlernbaren theoretischen Prinzipien, die Möglichkeiten ihrer Anwendung und die Bedingungen ihrer praktischen Durchsetzbarkeit hin untersucht. Ergebnis ist eine gründliche Neubestimmung des Verhältnisses von Theorie, Anwendung und Praxis. Die historisch bekannten Wirtschaftsschulen und -richtungen werden auf ihren Geltungsanspruch hinterfragt. Politische und ideologische Rahmenbedingungen werden in ihrem Einfluß auf Privat- und Gemeinwirtschaft erläutert und Modelle zur Überprüfung von Wirtschaftspolitik und Wirtschaftsethik aufgezeigt.

Aus dem Inhalt: Wissenschaftsanspruch, Definition und Methoden der Ökonomie - Einfluß von politischen und ideologischen Rahmenbedingungen . Privat- und Gemeinwirtschaft - Wirtschaftsschulen und Wirtschaftsmodelle . Wirtschaftsrecht und Wirtschaftsethik - Ökonomische Begriffsbildung und Wissenschaft · Ökonomie und Philosophie

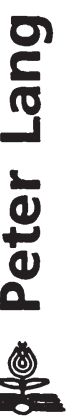

Frankfurt/M - Berlin - Bern - New York - Paris - Wien

Auslieferung: Verlag Peter Lang AG

Jupiterstr. 15, CH-3000 Bern 15

Telefax (004131) 9402131

*inklusive Mehnwertsteuer

Preisänderungen vorbehalten 
Joachim Nagel - 978-3-631-75238-8

Downloaded from PubFactory at 01/11/2019 06:47:33AM

via free access 